\title{
Measurement of the Top Quark Mass with the Collider Detector at Fermilab
}

Koji Sato

February 2005 



\title{
Measurement of the Top Quark Mass with the Collider Detector at Fermilab
}

\author{
Koji Sato \\ (Doctoral Program in Physics)
}

\author{
Submitted to the Graduate School of \\ Pure and Applied Sciences \\ in Partial Fulfillment of the Requirements \\ for the Degree of Doctor of Philosophy in \\ Science \\ at the \\ University of Tsukuba
}





\section{Abstract}

We present a measurement of the top quark mass using $t \bar{t}$ pair creation events decaying into the lepton + jets channel in $p \bar{p}$ collisions at $\sqrt{s}=1.96 \mathrm{TeV}$. The data sample used in this analysis was collected with the Collider Detector at Fermilab (CDF) in Tevatron Run II during the period from March 2002 through August 2003.

Tagging the jets originating from $b$ quarks not only helps reduce the background events but also plays an important role in the correct reconstruction of the $t \bar{t}$ event. Our analysis subdivides the candidate events into three categories - 0,1 and 2 tag channels - by the numbers of $b$-tagged jets in the events. The categorization of the events has been optimized for the best sensitivity for the top quark mass. The accumulated integrated luminosities for each channel were : $193 \mathrm{pb}^{-1}$ for $0 \mathrm{tag}$, and $162 \mathrm{pb}^{-1}$ for 1 and 2 tag channels.

We found eleven $t \bar{t}$ candidate events in $2 \mathrm{tag}$, seventeen in $1 \mathrm{tag}$ and forty events in 0 tag channel. We extracted a top mass of $180.9{ }_{-6.0}^{+6.4}$ (stat) \pm 5.8 (syst) $\mathrm{GeV} / c^{2}$ using 2 tag candidate events, and a combined top mass of $177.2_{-4.7}^{+4.9}$ (stat) \pm 6.6 (syst) $\mathrm{GeV} / c^{2}$ using the total sixty-eight candidate events in the three channels. 


\section{Acknowledgments}

This thesis could not have been completed without the support and encouragement of countless people. I would like to mention some of them here.

I thank my adviser, Professor Shin-Hong Kim, for the invaluable guidance he has offered me during the whole period of this study. Especially his insights and ideas for my subject were always amazing, and my study totally depended on them. Indescribable thanks are due to Professor Takasumi Maruyama and Tomonobu Tomura for all the hard work they shared with me as well as lots of precious advice. These three people were always prepared to spare their time to discuss with me and guide me in my analysis. I really appreciate their constant support and their tolerance for my slow pace at work.

I would like to thank all members of the CDF top mass template group. I owe a lot to Luc Demortier, Un-Ki Yang, Guram Chlachidze, Jean-Francois Arguin, Erik Brubaker and YoungKee Kim for their useful help at critical times of the analysis. Discussions with these people turned out to be very beneficial. Special thanks are due to Luc Demortier, who constantly encouraged me through my study. I am also grateful to the top group conveners of CDF, Jaco Konigsberg, Evelyn Thomson, Doug Glenzinski and Eva Halkiadakis, who were very supportive and pushed me forward in my study.

I am thankful to all the members of the high energy experiment group of University of Tsukuba. Especially, Professor Koji Takikawa, Professor Fumihiko Ukegawa, Professor Kazuhiko Hara, Professor Yoshihiro Seiya, Yuji Takeuchi and Hirohuki Matsunaga provided me the opportunity to get involved in the CDF experiment and supported my study in many ways. I also thank people in the Japan group of the CDF collaboration. They were sometimes good friends, and sometimes good colleagues in the research. Such friendships were invaluable especially during my visits abroad at Fermilab.

Lastly, no words are enough to describe my thanks to my parents, Eiji and Yukiko Sato. They have been a bottomless well of encouragement for me, and were always ready to support me in every possible way. 


\section{Contents}

$\begin{array}{ll}\text { Abstract } & 1\end{array}$

$\begin{array}{ll}\text { Acknowledgments } & 3\end{array}$

1 Introduction $\quad 1$

2 Experimental Design $\quad 11$

2.1 The Accelerator Complex . . . . . . . . . . . . . . . . . . 11

2.1.1 Proton Production and Boosting . . . . . . . . . . . . 14

2.1 .2 Main Injector . . . . . . . . . . . . . . . . . . . 14

2.1.3 Antiproton Production . . . . . . . . . . . . . . 15

2.1.4 Recycler Ring . . . . . . . . . . . . . . . . . . . 15

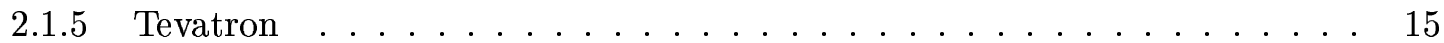

2.1.6 Beam Monitors . . . . . . . . . . . . . . . . 16

2.2 The CDF Detector . . . . . . . . . . . . . . . . 16

2.3 Tracking System _ . . . . . . . . . . . . . . . . 18

2.3 .1 Silicon Vertex Detector f . . . . . . . . . . . 18

2.3.2 Central Outer Tracker . . . . . . . . . . . . . . . . . 18

2.3 .3 Time of flight . . . . . . . . . . . . . . . . . 19

2.3 .4 Magnet . . . . . . . . . . . . . . . . . 19

2.4 Calorimetry . . . . . . . . . . . . . . . . . . . . 19

2.4 .1 Overview . . . . . . . . . . . . . . . . 19

2.4 .2 Central Calorimeter . . . . . . . . . . . . . . 20

2.4 .3 Plug Calorimeter Upgrade . . . . . . . . . . . . . . . . . . 21

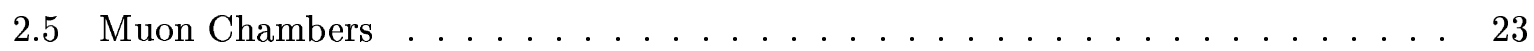

2.5.1 Central Muon Detectors . . . . . . . . . . . . . 25

2.5 .2 Intermediate Muon Detectors . . . . . . . . . . . . 26

2.6 Data Acquisition and Trigger . . . . . . . . . . . . . . 26

2.6 .1 Level 1 trigger . . . . . . . . . . . . . . . . . 26

2.6 .2 Level 2 trigger $\ldots \ldots \ldots \ldots \ldots \ldots \ldots$ 
2.6 .3 Level 3 trigger . . . . . . . . . . . . . . . . 28

2.6 .4 Online Monitoring . . . . . . . . . . . . . . 29

3 Particle Identifications $\quad 31$

3.1 Lepton Identification . . . . . . . . . . . . . . . . . . . . 31

3.1 .1 Identification of a Central Electron . . . . . . . . . . . . . . 31

3.1 .2 Identification of a Plug Electron . . . . . . . . . . . . . 32

3.1 .3 Identification of a Muon . . . . . . . . . . . . . . . . 33

3.2 Jet Identification and Corrections . . . . . . . . . . . . . . . . . 33

$3.3 \quad b$-Jet Tagging . . . . . . . . . . . . . . . . . . . 35

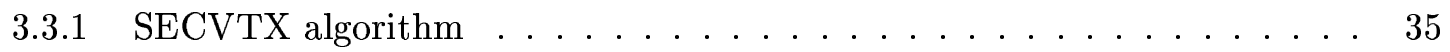

3.3 .2 Jet Probability Tagging . . . . . . . . . . . . . . . . 36

3.4 Missing Transverse Energy; t $_{T} \ldots \ldots \ldots \ldots \ldots \ldots \ldots$

4 Data Sample and Event Selection $\quad 43$

4.1 Data Sample . . . . . . . . . . . . . . . . . . . . . 43

4.2 Monte Carlo samples . . . . . . . . . . . . . . . . . . . . . 44

4.3 Event Selection . . . . . . . . . . . . . . . . . . . 44

4.4 Subdividing the Sample . . . . . . . . . . . . . . . . . . 45

5 Top Quark Mass Reconstruction in each Event $\quad 49$

5.1 Correcting Measured Quantities . . . . . . . . . . . . . . . . . . . . 49

5.1 .1 Lepton Corrections . . . . . . . . . . . . . . . . . . . . . 49

5.1 .2 Generic Jet Corrections . . . . . . . . . . . . . . . 50

5.1 .3 Top-Specific Jet Corrections . . . . . . . . . . . . . . . . . . 50

5.1.4 Additional Jets, Unclustered Energy, and Missing Energy . . . . . . . . 52

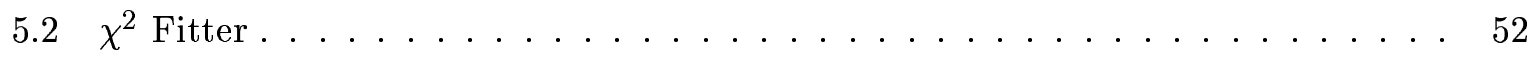

$5.3 \quad B$-Tagging Conditions and the Choice of the Fit Results . . . . . . . . . . 53

5.4 Characteristics of the Sub-samples . . . . . . . . . . . . . . 55

5.4 .1 Efficiency of Sample Selection . . . . . . . . . . . . . . 56

5.4 .2 Study of Combinatorial Background . . . . . . . . . . . . 56

5.4.3 Dependence of the Event Reconstruction on the Top Quark Mass _ . . . 60

6 Mass Templates $\quad 65$

$6.1 t \bar{t}$ Signal Templates . . . . . . . . . . . . . . . . . . . . 65

6.2 Background Templates . . . . . . . . . . . . . . . . . 66

6.2.1 Estimation of the Number of Background Events . . . . . . . . . 66

6.2 .2 Background Template Building . . . . . . . . . . . . . 82

6.3 Summary of the Templates $\ldots \ldots \ldots \ldots \ldots \ldots$ 
7 Determination of the Top Quark Mass $\quad \mathbf{8 7}$

7.1 Determination of the Top Quark Mass . . . . . . . . . . . . . . 87

7.2 Method of the Pseudo-experiments . . . . . . . . . . . . . . 88

8 Sensitivity Study $\quad 91$

8.1 Optimization of the Jet Probability Cut . . . . . . . . . . . . . . 92

8.1.1 Expected Number of Events for 1 and 2 Tag Samples . . . . . . . . . 92

8.1.2 Templates for the Mass Fitting at Various Jet Probability Cuts . . . . . . 93

8.1.3 Statistical Sensitivity with 1 and 2 tag samples . . . . . . . . . . 95

8.1 .4 Adding 0 tag sample . . . . . . . . . . . . . . . 96

8.1.5 Summary of Sensitivity Study . . . . . . . . . . . . . 100

8.2 Effect of the Event Recycling . . . . . . . . . . . . . . . . . 101

9 Validation of the Measurement Method $\quad 103$

9.1 Bias to the Center Value . . . . . . . . . . . . . . . . . 103

9.2 Bias to the Statistical Error . . . . . . . . . . . . . . 105

10 Systematic Uncertainties $\quad 111$

10.1 Scheme of Estimation . . . . . . . . . . . . . . . 111

10.2 Generator . . . . . . . . . . . . . . . . . . . 112

10.3 Jet Resolution . . . . . . . . . . . . . . . . . . . . . 113

10.4 Jet Energy Scale Uncertainty . . . . . . . . . . . . . . . . . . . . . . . . . . . . . . . . . . . . .

10.5 Initial and Final State Gluon Radiation (ISR / FSR) . . . . . . . . . . . . 114

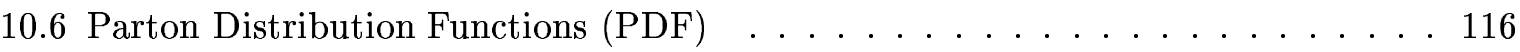

10.7 Background Shape . . . . . . . . . . . . . . . . . . 117

$10.8 b$-Tagging Scale Factor . . . . . . . . . . . . . . . . . 120

10.9 Summary of the Systematic Uncertainties . . . . . . . . . . . . . . 121

11 Results and Discussions $\quad 123$

11.1 Candidate Events . . . . . . . . . . . . . . . . 123

11.2 Di-jet mass $m_{j j}$ for the Selected 2 Tag Events . . . . . . . . . . . . . 124

11.3 Results of the Likelihood Fit . . . . . . . . . . . . . 125

11.4 Check of the Result with Pseudo-Experiments . . . . . . . . . . . . . 128

11.5 Investigation into the 1 Tag Sample . . . . . . . . . . . . . . 129

11.6 Scale Factor for the Statistic Errors . . . . . . . . . . . . . . 129

12 Conclusion $\quad 135$

$\begin{array}{ll}\text { References } & 137\end{array}$ 


\section{List of Figures}

$1.1 t \bar{t}$ production processes at Tevatron. ................. 5

1.2 $t \bar{t}$ production cross section measured at CDF in Run $\mathrm{I}(\sqrt{s}=1.8 \mathrm{TeV})$ and Run II $(\sqrt{s}=1.96 \mathrm{TeV})$. The band shows the theoretical prediction of the Standard

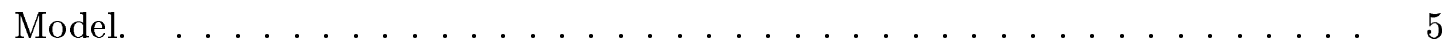

1.3 A diagram for $t \bar{t}$ production by $q \bar{q}$ annihilation and its decay into lepton + jets channel. . . . . . . . . . . . . . . . . . 6

1.4 Summary of top quark mass measured by using Run I Tevatron data. di- $1,1+j$ and all-j stand for dilepton, lepton+jets and all-hadronic channels respectively. . 7

1.5 Virtual top quark loops contributing to $W$ and $Z$ masses. . . . . . . . 8

1.6 Virtual Higgs boson loops contributing to $W$ and $Z$ masses. . . . . . . . . . 8

1.7 The $68 \%$ confidence level contour for $m_{W}$ and $m_{\text {top }}$. Contours for indirect and direct measurements are plotted as well as the aimed for contour of Tevatron Run II. Also shown is the Standard Model relationship for the masses as a function of the Higgs mass. . . . . . . . . . . . . . . . . . . . .

$1.8 \Delta \chi^{2}=\chi^{2}-\chi_{\min }^{2}$ vs Higgs boson mass $M_{H}$. The line shows the best fit. The band represents an estimate of the theoretical error due to missing higher order corrections. The vertical band shows the $95 \%$ confidence level exclusion limit on $M_{H}$ from the direct search. The dashed curve is the result obtained using the evaluation of $\Delta \alpha_{h a d}^{(5)}\left(M_{Z}^{2}\right)$ from $[20] \ldots \ldots \ldots \ldots \ldots$

2.1 Elevation view of one half of the CDF II detector . . . . . . . . . . . 17

2.2 Cross section of the CDF II plug calorimeter $\ldots \ldots \ldots \ldots$

$2.3 \eta$ and $\phi$ coverage of the Run II muon systems $\ldots \ldots \ldots \ldots$

2.4 Block diagram of the CDF II Trigger . . . . . . . . . . . . . . 27

2.5 Design of the CDF online consumer framework. . . . . . . . . . . . 30 
3.1 The correction scales for jet energy corrections. The left plots are the corrections applied to jets in collision data, and right plots are for Monte Carlo. The top plots are when we correct up to level 4 , and the bottom plots are for level 5 . Correction scales for jets with several different $E_{T}$ are shown. These plots suppose the jets have EM energy fraction of 0.5 and the events have only one vertex. . . . . . 35

3.2 Jet Probability distributions from prompt, charm and bottom jets. . . . . . . 37

3.3 (Left) tracks from a primary vertex. (Right) tracks from a secondary vertex. . . 38

3.4 (Left) signed impact parameter distribution for tracks from primary vertex. (Right) signed impact parameter distribution for tracks from secondary vertex. . . . . . . 39

3.5 The positive part of the impact parameter significance of tracks in primary jets. Track probability is defined as the shaded area for given impact parameter significance when the total area is normalized to $1 . \ldots \ldots \ldots$. . . . . . 40

3.6 Definition of jet probability for a jet which has two good quality tracks with positive signed impact parameters in it. For a case where the tracks have track probabilities as shown in the dot, the jet probability is defined as the shaded area. 41

4.1 Subdividing the data sample. . . . . . . . . . . . . . . . 47

4.2 Event display for one of our candidate events found in data. Top : Energy deposit in each calorimeter tower is shown in the $\eta$ vs. $\phi$ plane. Bottom : The reconstructed tracks found in the jets. The reconstructed secondary vertices are also displayed. . . . . . . . . . . . . . . . . 48

5.1 Upper plot: jet response, $\left(p_{T}^{\text {parton }}-p_{T}^{\text {jet }}\right) / p_{T}^{\text {jet }}$, as a function of $p_{T}^{j e t}$ for $b$-jets and $W$-jets after only generic corrections are applied. Lower plot: jet response as a function of $\eta^{j e t}$ after applying top-specific corrections that depend only on $p_{T}^{j e t}$. . 51

5.2 Distribution of $\chi^{2}$ in 0 tag sample, before the $\chi^{2}$ cut for $t \bar{t}$ Monte Carlo events. Distributions for correct and wrong combinations are also shown. $\quad \ldots . . . .54$

5.3 Distribution of $\chi^{2}$ in 1 tag sample, before the $\chi^{2}$ cut for $t \bar{t}$ Monte Carlo events. Distributions for correct and wrong combinations are also shown. . . . . . . . 54

5.4 Distribution of $\chi^{2}$ in 2 tag sample, before the $\chi^{2}$ cut for $t \bar{t}$ Monte Carlo events. Distributions for correct and wrong combinations are also shown. . . . . . . 55

5.5 The invariant mass distributions of two jets assigned to light flavor partons by the top mass reconstruction module in Monte Carlo $t \bar{t}$ and $W b \bar{b}$ events. Histogram for $W b \bar{b}$ is scaled by a factor of 50 to be viewable. Hatched areas show the events

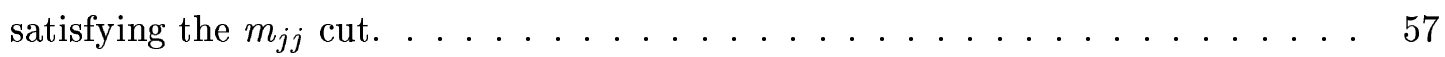


5.6 The reconstructed top mass distributions after applying all cuts in the $m_{t}=$ $175 \mathrm{GeV} / c^{2}$ Monte Carlo sample. For each plot, the solid histogram is for all the events, and the dashed histogram is for events where the $t \bar{t}$ decay was correctly reconstructed. The numbers in the right top box are the fraction of correct reconstruction and the RMS of the histogram for all the events (larger histogram). On top from left : samples with zero, $\geq 1$, and two $b$-jets tagged by SECVTX tagger after $\chi^{2}$ cut. On bottom : sample with two $b$-jets tagged by SECVTX and Jet Probability taggers after $\chi^{2}$ cut (left) and $m_{j j}$ cut (right). . . . . . . . . 58

5.7 Efficiency of our event selection for each sub-sample as a function of the top quark mass. . . . . . . . . . . . . . . . . . . . . . .

5.8 Reconstructed top mass distributions for different top quark masses are shown in solid histograms for 0 tag sample. The distributions of the correctly reconstructed events are also shown in dashed histograms. From left on top column : $m_{\text {top }}=150,165$ and $175 \mathrm{GeV} / c^{2}$, from left on bottom column : $m_{\text {top }}=180,190$ and $205 \mathrm{GeV} / c^{2}$. In the top-right box in each plot are the fraction of correct combination, along with the RMS of the solid histogram. . . . . . . . . .

5.9 Reconstructed top mass distributions for different top quark masses are shown in solid histograms for 1 tag sample. The distributions of the correctly reconstructed events are also shown in dashed histograms. From left on top column : $m_{\text {top }}=150,165$ and $175 \mathrm{GeV} / c^{2}$, from left on bottom column : $m_{\text {top }}=180,190$ and $205 \mathrm{GeV} / c^{2}$. In the top-right box in each plot are the fraction of correct combination, along with the RMS of the solid histogram. . . . . . . . . .

5.10 Reconstructed top mass distributions for different top quark masses are shown in solid histograms for 2 tag sample. The distributions of the correctly reconstructed events are also shown in dashed histograms. From left on top column : $m_{\text {top }}=150,165$ and $175 \mathrm{GeV} / c^{2}$, from left on bottom column : $m_{\text {top }}=180,190$ and $205 \mathrm{GeV} / c^{2}$. In the top-right box in each plot are the fraction of correct combination, along with the RMS of the solid histogram. . . . . . . . .

5.11 The fraction of events with correct reconstruction in each sample is plotted as a function of the top quark mass. . . . . . . . . . . . . . . . .

6.1 Signal templates for the 0 tag sample for input masses from $150 \mathrm{GeV}$ up to $205 \mathrm{GeV}$ along with the parametrized signal shape functions. . . . . . . . . .

6.2 Signal templates for the 1 tag sample for input masses from $150 \mathrm{GeV}$ up to $205 \mathrm{GeV}$ along with the parametrized signal shape functions. . . . . . . . . . .

6.3 Signal templates for the 2 tag sample for input masses from $150 \mathrm{GeV}$ up to $205 \mathrm{GeV}$ along with the parametrized signal shape functions. . . . . . . . .

6.4 Regions are defined in lepton isolation vs $\mathbb{E}_{T}$ plane in order to estimate the non- $W$ background. Region $\mathrm{D}$ is the signal region. . . . . . . . . . . . . 71 
6.5 One of the production processes for $W b \bar{b}$ in the leading order. . . . . . . . . 74

6.6 One of the production processes for $W b \bar{b}$ in the next to leading order. . . . . . 74

$6.7 \quad m_{j j}$ distributions for $W b \bar{b}$ (left) and $W c \bar{c}$ (right) . . . . . . . . . . 76

6.8 Diagrams of single top production at Tevatron. Off-mass shell $W$ production

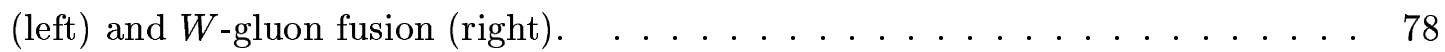

6.9 Background templates for 0 tag (top) 1 tag (bottom left) and 2 tag (bottom right) samples. Contribution from each different process is shown in different color for

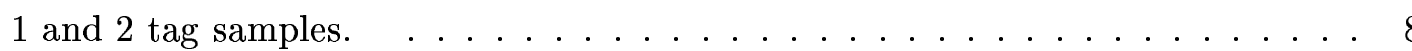

6.10 Background template along with the background shape function. Left-top: the background template for the no-tag, right-top: for single tag, bottom: for the

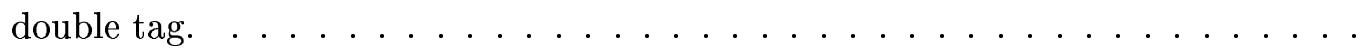

6.11 The obtained functions for signal events with various true top masses. The parametrized background shape is also shown. Functions are normalized to the equal area. Top figure shows 0 tag case, Left figure shows 1 tag case, while right figure shows the 2 tag case. . . . . . . . . . . . . 86

8.1 Upper and lower statistical error on measured top quark mass in pseudo-experiments. Mean values for upper and lower error are also shown. . . . . . . . . . . 91

8.2 Number of signal and background events as a function of the Jet Probability cut. 94

8.3 2 tag signal templates for different cut values on Jet Probability. The cut values are from left on top column : 0.00 (the case where Jet Probability is not used), 0.01 and 0.02 from left on bottom column : $0.05,0.1$ and 0.2 . In the top-right box in each plot are the fraction of correct combination, along with the RMS of the solid histogram. . . . . . . . . . . . . .

8.4 1 tag signal templates for different cut values on Jet Probability. The cut values are from left on top column : 0.00 (the case where Jet Probability is not used), 0.01 and 0.02 from left on bottom column : $0.05,0.1$ and 0.2 . In the top-right box in each plot are the fraction of correct combination, along with the RMS of the solid histogram. . . . . . . . . . . . . . . 96

8.5 Eighteen parameters for the top mass template at the various cut values on Jet Probability for $1 \mathrm{tag}$ sample. . . . . . . . . . . . . . 97

8.6 Eighteen parameters for the top mass template at the various cut values on Jet Probability for 2 tag sample. . . . . . . . . . . . . . . 98

8.7 Statistical sensitivity of 1 and 2 tag samples for integrated luminosity of $200 \mathrm{pb}^{-1}$ as a function of the Jet Probability cut. Left: expected lower statistical error. Right: expected upper statistical error. JPcut $=10^{-4}$ points show the case where only SECVTX tagger is used, and open circle corresponds to $\geq 1$ tag case. $\quad$. . . 99 
8.8 Statistical sensitivity of combined fit of 1 and 2 tag samples for integrated luminosity of $200 \mathrm{pb}^{-1}$ as a function of the Jet Probability cut. Left: expected lower statistical error Right: expected upper statistical error. JPcut $=10^{-4}$ point shows the case where only SECVTX tagger is used, and open circle corresponds to $\geq 1$ tag case. . . . . . . . . . . . . . . . . . .

8.9 Expected statistical sensitivity of the top mass measurement combining 0,1 and 2 tag samples for integrated luminosity of $200 \mathrm{pb}^{-1}$ as a function of the cut threshold on Jet Probability. Left: lower error. Right: upper error. JPcut $=10^{-4}$ point shows the combined results using only SECVTX, and the open circle corresponds to $\geq 1$ tag case (inclusive SECVTX sample measurement). . . . . . . . . 100

8.10 Upper and lower statistical error on measured top quark mass in pseudo-experiments for with and without the event recycling. Mean values for upper and lower errors are also shown. . . . . . . . . . . . . . . . . . . . 101

9.1 Center value returned from the likelihood fitter in pseudo-experiments assuming the top mass of $175 \mathrm{GeV} / c^{2}$. . . . . . . . . . . . . . . 104

9.2 Fitted output mass as a function of the top mass input for 0 tag (top-left), 1 tag (top-right), 2 tag (bottom-left) and combined fit (bottom-right). The line shows

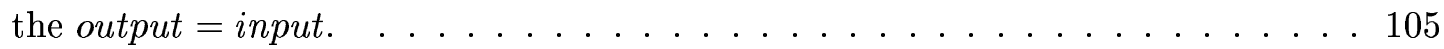

9.3 Difference between the output and the input masses is plotted against the input top mass for 0 (top-left), 1 (top-right), 2 tag sample (bottom-left) and combined fit (bottom-right). The line shows the output =input. . . . . . . . 106

9.4 Pull in pseudo-experiments assuming the top mass of $175 \mathrm{GeV} / c^{2}$. A fit to Gaussian function is also shown. . . . . . . . . . . . . . . 107

9.5 The mean of the pull distribution as a function of the input top mass for 0 (topleft), 1 (top-right), 2 tag sample (bottom-left) and combined fit (bottom-right).

9.6 Width of the pull distribution as a function of the input top mass for 0 (top-left), 1 (top-right), 2 tag sample (bottom-left) and combined fit (bottom-right). . . . 109

10.1 Signal template constructed with HERWIG and PYTHIA Monte Carlo events for 2 tag sample (left), and the measured top quark mass in pseudo-experiments by generating pseudo-events due to this template (right). . . . . . . . . . 112

10.2 The size of the uncertainty due to different $\alpha_{S}$, different PDF groups, and uncertainty in the CTEQ eigenvectors (left plots). The measured top quark mass in pseudo-experiments is plotted as a function of the PDF number defined in text (right plots). Plots are for combined fit, 0,1 and 2 tag samples from top. . . . 118 
11.1 Invariant mass of the two light flavor jets before (left) and after (right) the $\chi^{2}$ cut. Histograms show the Monte Carlo distribution $\left(m_{t}=175 \mathrm{GeV} / c^{2}\right)$ while the crosses show the data. . . . . . . . . . . . . . . . . 125

11.2 Result of the likelihood fit to the data sample for 0 tag (top-left), 1 tag (top-right), 2 tag (bottom-left) and the combined fit of these three channels (bottom-right). The histogram shows the reconstructed mass of the data events for each sample. The distribution shape and the background contribution corresponding to the best fit are shown in lines. In the top-right box of each plot is the likelihood curve used in the measurement of the mass of the top quark. For the combined fit, we only show the likelihood curve. . . . . . . . . . . . . . . . 126

11.3 Result of the likelihood fit to the data sample for 0 tag (top-left), 1 tag (top-right), $2 \mathrm{tag}$ (bottom-left) and the combined fit of these three channels (bottom-right). The background constraint is turned off. The histogram shows the reconstructed mass of the data events for each sample. The distribution shape and the background contribution corresponding to the best fit are shown in lines. In the top-right box of each plot is the likelihood curve used in the measurement of the mass of the top quark. For the combined fit, we only show the likelihood curve.

11.4 Distribution of the upper and lower statistical errors on top quark mass for 0 tag sample measurement obtained in 1000 pseudo-experiments assuming top mass of $175 \mathrm{GeV} / c^{2}$. The arrow indicates the value in data measurement. . . . . . . . 128

11.5 Distribution of the upper and lower statistical errors on top quark mass for 1 tag sample measurement obtained in 1000 pseudo-experiments assuming top mass of $175 \mathrm{GeV} / c^{2}$. The arrow indicates the value in data measurement. . . . . . . 129

11.6 Distribution of the upper and lower statistical errors on top quark mass for 2 tag sample measurement obtained in 1000 pseudo-experiments assuming top mass of $175 \mathrm{GeV} / c^{2}$. The arrow indicates the value in data measurement. . . . . . . . 130

11.7 Distribution of the upper and lower statistical errors on top quark mass for measurement by combined fit obtained in 1000 pseudo-experiments assuming top mass of $175 \mathrm{GeV} / c^{2}$. The arrow indicates the value in data measurement. . . . . . . 131

11.8 Fit results with background constrained using only 4 jet events in the tagged samples. Top left : 0 tag sample, top right : 1 tag sample, bottom left : 2 tag sample, bottom right : combined fit of the three samples. . . . . . . . . . 131

11.9 The percentage of the pseudo-experiments which have the input top mass within the statistical errors around the output center values (on the vertical axis). The scale factor applied to the statistical error from the likelihood fit is plotted on the horizontal axis. Left plot is for the $2 \mathrm{tag}$ sample and the right plot is for the combined fit. . . . . . . . . . . . . . . . . 134 
12.1 Results of top quark mass measurements using different analysis methods at CDF Run II. The result presented in this dissertation is referred to as $M_{\text {reco }}$. . . . 136 


\section{List of Tables}

1.1 Fundamental Particles of the Standard Model. . . . . . . . . . . . . . . 3

1.2 Comparison of masses of Standard Model quarks. . . . . . . . . . . . . . . . . 3

1.3 Branching Ratios for $t \bar{t}$ decay modes in standard model coupling. $q$ stands for a $u, d, c$ or $s$ quark. Decay modes are categorized into four channels: All-jets, Lepton+jets, Di-lepton and $\tau$ channels. . . . . . . . . . 6

2.1 Evolution of Tevatron parameters . . . . . . . . . . . . . . . . . . . . . . . . . .

2.2 Evolution of Tevatron parameters . . . . . . . . . . . . . . . . . . . . . . . . . .

2.3 Calorimeter segmentation . . . . . . . . . . . . . 20

2.4 Characteristics of the CDF II calorimeter . . . . . . . . . . . . . 21

2.5 Parameters of muon detection at CDF . . . . . . . . . . . . . . 24

3.1 Summary of the Jet Probability tagger calibration. The scale factor is the ratio of efficiencies for heavy flavor jets between data and Monte Carlo. The efficiency for heavy flavor measured in data is shown here. The mistag rate corresponds to the efficiency of this tagger for light flavor jets. . . . . . . . . . . 40

5.1 The efficiencies of our event selection. See text for detailed description. . . . . 56

5.2 Efficiency of $m_{j j}$ cut for each category of Monte Carlo events. . . . . . . . . . 57

5.3 The percentage (\%) of the events that come into each category of mis-assignment in the event reconstruction. See text for the definition of the categorization. The sum of the six categories is $100 \%$, and the "total" shows the number of Monte Carlo events corresponding to $100 \%$. Besides 0, 1 and 2 tag samples, we summarize the results for 2 tag sample without $m_{j j}$ cut and 2 SECVTX tag sample. We also show results for 1 SECVTX tag sample divided into recycled

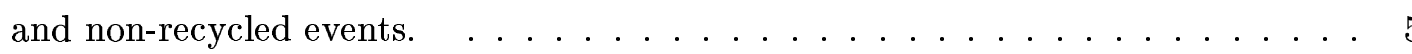

$6.1 \alpha$ parameters obtained in the signal template fitting for the 0 (left column), 1 (middle) and 2 (right) tag samples. . . . . . . . . . . . 70

6.2 The number of events in the regions defined above for before $b$-tag, $\geq 1$ SECVTX tag, 2 tag and 2 tag after $\chi^{2}$ and $m_{j j}$ cuts. . . . . . . . . . 72 
6.3 Estimated number of background with $\geq 1$ SECVTX $b$-tag required. These are the numbers before $\chi^{2}$ cut. . . . . . . . . . . . . . . . 73

6.4 Summary of the background estimation for $2 \mathrm{tag}$ sample. $N_{162}^{\geq 1 \mathrm{tag}} \mathrm{pb}^{-1}$ is the sum of numbers of $W+3.5$ jets and $W+4$ jets in Table 6.3. $\varepsilon_{2 n d t a g}, \varepsilon_{\chi^{2} c u t}$ and $\varepsilon_{m_{j j}}$ are efficiencies for our secondary tag, $\chi^{2}$ cut and $m_{j j}$ cut respectively. $\varepsilon_{\text {total }}$ refers to the total efficiency of these three cuts. Errors on these four efficiencies come purely from Monte Carlo statistics. Multiplying $N_{162}^{\geq 1 t a g}$ pb $\varepsilon_{\text {total }}$, we obtain the estimated number of background events in 2 tag sample at an integrated luminosity of $162 \mathrm{pb}^{-1}$. $\varepsilon_{m_{j j}}$ is $100 \%$ for $W c$ Monte Carlo sample because no event was rejected at this cut due to low statistics. The error on this $\left({ }_{-8.14}^{+0}\right)$ comes from the Poisson statistics. . . . . . . . . . . . . . 75

6.5 The estimation of the number of $\geq 1$ SECVTX tagged background events passing through $\chi^{2}<9$ cut in the 3.5 jet bin. . . . . . . . . . . . 80

6.6 The estimation of the number of $\geq 1$ SECVTX tagged background events passing through $\chi^{2}<9$ cut in the 4 jet bin. . . . . . . . . . . . . 80

6.7 Summary of the Background Estimation for the Jet Probability cut of 0.05. . . 82

$6.8 \beta$ parameters obtained in the background template fitting for the zero, single and double tag samples. . . . . . . . . . . . . . . . 85

8.1 Expected number of $t \bar{t}$ signal events for different Jet Probability cuts assuming $200 \mathrm{pb}^{-1}$ for the integrated luminosity and $6.7 \mathrm{pb}$ for the theoretical cross section. "None" means we only use SECVTX tagging for the selection of 2 tag sample. These two Jet Probability cut values are those for which the algorithm is calibrated. . . . . . . . . . . . . . . . . . 93

8.2 Statistical sensitivity on the mass of top quark assuming integrated luminosity of $200 \mathrm{pb}^{-1}$ for several representative cuts on Jet Probability for measurement combining 1 and 2 tag samples. . . . . . . . . . . . . . . . 99

8.3 Expected statistical sensitivity on the mass of top quark with several representative cuts on Jet Probability for integrated luminosity of $200 \mathrm{pb}^{-1}$ in the measurement combining 0,1 and 2 tag samples. . . . . . . . . 100

10.1 Estimation of systematic uncertainty due to the generator modeling. . . . . . 113

10.2 Systematic uncertainty due to the jet energy correction. . . . . . . . . . . 115

10.3 Systematic uncertainty due to the initial state gluon radiation. . . . . . . . 116

10.4 Systematic uncertainty due to the and final state radiation. . . . . . . . . . 116

10.5 The estimation of the uncertainty due to PDF . . . . . . . . . . . 117

10.6 Systematic uncertainty due to the background fraction of each source. . . . . . 119

10.7 Top mass results in pseudo-experiments assuming different $Q^{2}$ for $W b \bar{b}$ background process in the construction of background template. . . . . . . . 119 
10.8 Top mass shift due to different 0 tag background templates. . . . . . . . . 120

10.9 Total uncertainty due to background shape modeling. . . . . . . . . . . . . 120

10.10Top mass measured in pseudo-experiments supposing nine different combination of varied absolute values of scale factors for the SECVTX and Jet Probability. . 121

10.11Top mass measured in pseudo-experiments supposing nine different combination of varied $E_{T}$ dependence of scale factors for the SECVTX and Jet Probability. $\quad 122$

10.12 Top mass shift on $b$-tag scale factor variations. . . . . . . . . . . . . 122

10.13The summary of the systematic uncertainties. We assume these are uncorrelated. 122

11.1 The eleven events in $2 \mathrm{tag}$ sample used in the measurement of the top quark mass. Jets with their $E_{T}$ values tagged with ${ }^{S}$ are SECVTX tagged and with $J$ are tagged by Jet Probability. The jet $E_{T}$ shown here are after the generic correction at level 5. . . . . . . . . . . . . . . . . . . 124

11.2 The six events rejected by the $\chi^{2}$ cut in the 2 tag sample selection. . . . . . . . 124

11.3 The twenty-six events are selected as 1 tagged candidates, including the events rejected in 2 tag event selection. Nine events shown in the bottom failed to pass the $\chi^{2}$ cut. Remaining seventeen events are to be used in the mass measurement for the 1 tag sample. Jets with their $E_{T}$ 's tagged with ${ }^{S}$ are SECVTX tagged and with ${ }^{J}$ are Jet Probability tagged. The jet $E_{T}$ shown here are after the generic correction at level 5. . . . . . . . . . . . . . . . . . 132

11.4 Forty-two events survived our kinematic cuts for the 0 tag selection criteria, including the $E_{T}^{j 4}>21 \mathrm{GeV}$. Two of these events were rejected at our $\chi^{2}$ cut, which are shown at the bottom of this table. Remaining forty events are used as 0 tag sample and are used for the mass measurement. . . . . . . . . . 133

11.5 Summary of the likelihood fit with and without constraint on the number of background events. The measured top quark mass and the background fraction is shown for each likelihood fit. The input background fraction is also shown for the fit with the background constraint. . . . . . . . . . . . . . 134 


\section{Chapter 1}

\section{Introduction}

Particle physics is an effort to expand the limits of human knowledge of nature by identifying the constituents of matter at the most fundamental level - elementary particles - and understand the interactions between these constituents. In the last few decades, it has been proven an efficient way in such scientific researches to steer together two accelerated beams of particles traveling in opposite directions and study the properties of the remnant particles that were produced in the collisions of the particles. Tevatron, located at Fermi National Accelerator Laboratory (Fermilab) in Batavia, IL in U.S.A., has been one of such particle colliders providing physicists opportunities to study particle physics in proton-antiproton $(p-\bar{p})$ collisions at the

highest energy in the world since 1987. The studies of the top quark has only been possible in the data taken in Tevatron collisions. Tevatron accelerator is designed so that the beams collide at two points along its circular length. At each points detector complexes are placed to accumulate data from these high energy particle collisions - CDF and D0 detectors. Tevatron accelerator operated during the periods 1987 - 1991 (Run 0) and 1992 - 1996 (Run I). After eight years of upgrading, it started the operation again, called Run II (2002 - ).

One of the most important roles of particle physics experiments at high energy conditions is to search for new particles which are too massive to be produced at previous experiments. CDF collaboration reported the evidence for top quark in 1994. In 1995, CDF collaboration, along with D0 collaboration, reported the discovery of the top quark in the $p \bar{p}$ collisions at the center-of-mass energy $\sqrt{s}=1.8 \mathrm{TeV}$ [1]. Another important challenge for the experimentalists is to refine the measurements of the properties of the discovered particles to the achievable best precision and feed the results as new inputs to the existing theories. One of the successful theories in the modern particle physics is called the Standard Model (SM).

\section{The Standard Model}

The Standard Model is a theory of fundamental particles, which has explained the phenomena observed in particle physics experiments for the last few decades and no clear sign of its con- 
tradiction with nature has been reported yet. It is a quantum field theory based on the gauge symmetry groups $S U(3)_{C} \times S U(2)_{L} \times U(1)_{Y}$. The first gauge group $S U(3)_{C}$ corresponds to the strong force described by Quantum Chromodynamics (QCD) [2], and the second and third gauge groups, $\mathrm{SU}(2)_{L} \times \mathrm{U}(1)_{Y}$, correspond to the symmetry of the electroweak interactions. $\mathrm{SU}(2)_{L}$ corresponds to the weak force and the V-A theory of weak interactions [3] and $\mathrm{U}(1)_{Y}$ corresponds to the electromagnetic force of Quantum Electrodynamics (QED) [4]. There is another known force in the nature, gravitation, but its interaction is too weak to be detected in the subatomic experiments. Therefore gravitation is not understood in terms of particle physics, and has not been included in the Standard Model.

The fundamental particles are categorized into three categories - leptons, quarks and gauge bosons. The leptons and quarks are the ultimate constituents of matter in the best knowledge of the modern science, and are fermion particles with spin 1/2 which obey the Pauli exclusion principle. Six types of leptons and six flavor of quarks have been known at present. Leptons are further categorized into two categories. Those with electric charge -1 - electron $\left(e^{-}\right)$, muon $\left(\mu^{-}\right)$and tau $\left(\tau^{-}\right)$, and those which are neutral in charge - neutrinos $\left(\nu_{e}, \nu_{\mu}\right.$ and $\left.\nu_{\tau}\right)$. The charged leptons interact through weak and electromagnetic interactions, while neutrinos only interact through weak interactions due to its lack of electric charge. Quarks are also categorized into two categories. Up-type quarks - up $(u)$, charm $(c)$ and top $(t)$ - with charge of $2 / 3$, and down-type quarks - down $(d)$, strange $(s)$ and bottom $(b)$ - with charge of $-1 / 3$. Besides their electric charges, quarks carry "color" charges (either red, green or blue) and experience strong interactions as well as the electromagnetic and weak interactions. Every lepton and quark has its own anti-particle which is identical in terms of mass and spin, but has opposite electric charge. Anti-particles are denoted by placing bars above the particle symbols. Leptons and quarks are often arranged in three generations of isospin doublets. The only difference between the different generations is - apart from the weak mixing angle - the different mass of the particles. So far, no convincing explanation has been made for the existence of this generation structure.

The Standard Model characterizes the interaction between the leptons and quarks as mediated by another category of particles. These mediator particles are bosons with internal spin of 1 , Bose-Einstein statistics and are called gauge bosons. The electromagnetic interaction is mediated by photon $(\gamma)$ and involve all the particles that have electric charge. The photon itself has no charge, and does not interact with itself. The weak interaction involves all leptons and quarks, and is mediated by weak bosons, $W^{ \pm}$and $Z^{0}$. The $W$ bosons have electric charges of either +1 or -1 while $Z^{0}$ boson is electrically neutral. These weak bosons are very massive and have masses of around $100 \mathrm{GeV} / c^{2}$. Due to their heavy masses, weak bosons can not travel a long distance, and the weak interaction is characterized by short distance. The strong interaction is mediated by eight different gluons. The gluons are massless, electrically neutral and carry color charge. Due to their internal color charge, gluons themselves interact through the strong interaction. 
Table 1.1 shows a summary of the properties of the fundamental particles. Table 1.2 sum-

\begin{tabular}{|c|cccccc|}
\hline \hline & 1st generation & 2nd generation & 3rd generation & Interaction & Charge (Q) & Spin \\
\hline Leptons & electron $\left(e^{-}\right)$ & muon $\left(\mu^{-}\right)$ & tau $\left(\tau^{-}\right)$ & EM, Weak & -1 & $1 / 2$ \\
& $e$-neutrino $\left(\nu_{e}\right)$ & $\mu$-neutrino $\left(\nu_{\mu}\right)$ & $\tau$-neutrino $\left(\nu_{\tau}\right)$ & Weak & 0 & $1 / 2$ \\
\hline Quarks & up $(u)$ & charm $(c)$ & top $(t)$ & EM, Weak, Strong & $+2 / 3$ & $1 / 2$ \\
& down $(d)$ & strange $(s)$ & bottom $(b)$ & EM, Weak, Strong & $-1 / 3$ & $1 / 2$ \\
\hline \hline & Name & Force & Coupling & Mass $\left(\mathrm{GeV} / \mathrm{c}^{2}\right)$ & Charge $(\mathrm{Q})$ & Spin \\
\hline Gauge & Photon $(\gamma)$ & EM & $10^{-2}$ & 0 & 0 & 1 \\
Bosons & W boson $(W)$ & Weak & $10^{-13}$ & 80.4 & \pm 1 & 1 \\
& Z boson $(Z)$ & Weak & $10^{-13}$ & 91.2 & 0 & 1 \\
& Gluon $(g)$ & Strong & 1 & 0 & 0 & 1 \\
\hline \hline
\end{tabular}

Table 1.1: Fundamental Particles of the Standard Model.

marizes the order of the mass of each quark [6]. Top quark has a huge mass compared to other quarks, which is about 35 times as heavy as the second heaviest quark, bottom.

The quarks and gluons behave approximately as free particles at high energies and their interactions can be calculated using perturbative theories. At low energies, these methods cannot be used because the coupling becomes strong. The quarks are bound together through the strong force (mediated by gluons) to form two types of hadrons: mesons and baryons. Mesons are bosons consisting of a quark an antiquark. For example, the $\pi^{+}$meson is composed of a $u$ and a $\bar{d}$ quarks. Baryons are fermions which consist of three quarks or anti-quarks. For example, the proton is composed of two $u$ quarks and a $d$ quark. Quarks and gluons are sometimes called "partons". In fact, the quarks form combinations in which the sum of their electric charges is an integer and the sum of their color charges is neutral (color-anticolor or (anti)red +(anti)blue + (anti)green). When a quark or gluon produced in high energy collisions, we can not observe it as such. It undergoes a complex process called fragmentation, where the parton emit gluons, gluons split into quark-antiquark pairs, and the quarks and antiquarks compose hadrons. In an experimental environment, we observe a parton produced in the high energy reaction as a bunch of hadrons flying in the same direction as the original parton. Such a collection of hadrons is called a jet.

"Higgs mechanism" explains how the particles obtain their masses in the standard model.

\begin{tabular}{|c|c|c|c|c|c|c|}
\hline \hline quark & $\mathrm{u}$ & $\mathrm{d}$ & $\mathrm{c}$ & $\mathrm{s}$ & $\mathrm{t}$ & $\mathrm{b}$ \\
\hline mass & $\sim 4 \mathrm{MeV} / c^{2}$ & $\sim 7 \mathrm{MeV} / c^{2}$ & $\sim 1.5 \mathrm{GeV} / c^{2}$ & $\sim 135 \mathrm{MeV} / c^{2}$ & $\sim 175 \mathrm{GeV} / c^{2}$ & $\sim 5 \mathrm{GeV} / c^{2}$ \\
\hline \hline
\end{tabular}

Table 1.2: Comparison of masses of Standard Model quarks. 
There are four vector bosons mediating the electroweak interactions $\left(\gamma, W^{ \pm}\right.$and $\left.Z^{0}\right)$, three of which with heavy masses. In order to theoretically explain the masses of weak bosons, a weak isospin doublet of new scalar field $\Phi=\left(\phi^{+}, \phi^{0}\right)$ is introduced with a potential :

$$
V\left(\Phi^{\dagger} \Phi\right)=\mu^{2}\left(\Phi^{\dagger} \Phi\right)+|\lambda|\left(\Phi^{\dagger} \Phi\right)^{2}
$$

where $\mu$ is the mass of the scalar field in the case of $\mu^{2}>0$ and $\lambda$ is the self coupling of the scalar field. In the Higgs mechanism, $\mu^{2}$ is let to be negative, with the potential having minimum at $\Phi=(0, v) / \sqrt{2} . \quad v=\sqrt{-\mu^{2} / \lambda}=\left(G_{F} \sqrt{2}\right)^{-1 / 2}=246 \mathrm{GeV}$ is referred to as the vacuum expectation value. When the field is expanded around the minimum, the electroweak symmetry is broken. This Electroweak Symmetry Breaking (EWSB) not only gives rise to the masses of $W^{ \pm}$and $Z$ bosons but also explains the non-zero masses of the fermion particles. At the same time, Electroweak Symmetry Breaking predicts a new boson called Higgs boson. Each quark and lepton has its Yukawa-coupling to the Higgs boson $G_{f}=\sqrt{2} m_{f} / v$, where "f" stands for fermion. Since the strength of this coupling to each fermion is proportional to its own mass, top quark plays an important role in the Higgs mechanism due to its uniquely heavy mass. The Higgs boson has not been yet discovered, and measurements of the properties of the top quark provides valuable insights into the properties of the Higgs boson.

The Standard Model has successfully explained many phenomena observed in particles physics experiments over several decades, and no clear sign of its contradiction with nature has been reported yet. On the other hand, it is fundamental to feed the correct input parameters in order for this theory to work. The mass of the top quark is one of such input parameters which the Standard Model is incapable of predicting. Especially interesting is the fact that the mass of the top quark is by far the heaviest among the elementary particles discovered so far. At the same time, it is an exciting coincidence that the mass of the top quark is very close to the vacuum expectation value of the Standard Model. The precision measurement of the top quark mass will play an important role in revealing the mechanism of the Electroweak Symmetry Breaking, and at the same time, closely related to the properties of the Higgs particle, the only particle yet to be discovered in the Standard Model.

\section{$t \bar{t}$ Pair Production and Decay}

The top quark is the heaviest elementary particle discovered so far. Its mass is about twice that of the $W$ and $Z$ bosons about 35 times as heavy as the second heaviest quark, $b$ quark. Because of its large mass, it can only be produced and studied in particle experiments performed at high energy region. Tevatron has been the only facility offering such an experimental environment in the history of the particle physics, colliding accelerated proton and anti-proton with the center-of-mass energy of $1.8 \mathrm{TeV}$ in Run I and $1.96 \mathrm{TeV}$ in Run II.

Figure 1.1 shows the $t \bar{t}$ production processes in a hadron collider. The production process shown on top is called $q \bar{q}$ annihilation and the bottom process is called $g g$ fusion. Calculation 
at the next-to-leading order predicts that the relative contributions to the $t \bar{t}$ production from these two processes at Tevatron Run II are $85 \%$ and $15 \%$ respectively. The total cross section
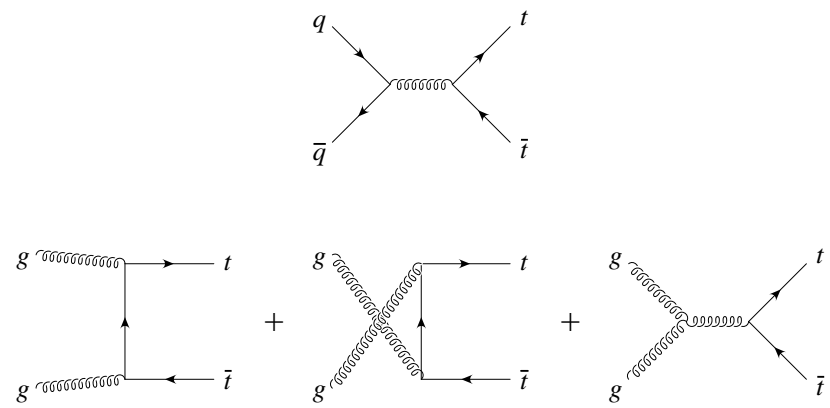

Figure 1.1: $t \bar{t}$ production processes at Tevatron.

for the pair production of top quark is theoretically calculated to be $6.7_{-0.9}^{+0.7} \mathrm{pb}[7,8]$. Figure 1.2 summarizes the total cross section measured at CDF experiment in Run I and Run II compared to the prediction of the Standard Model.

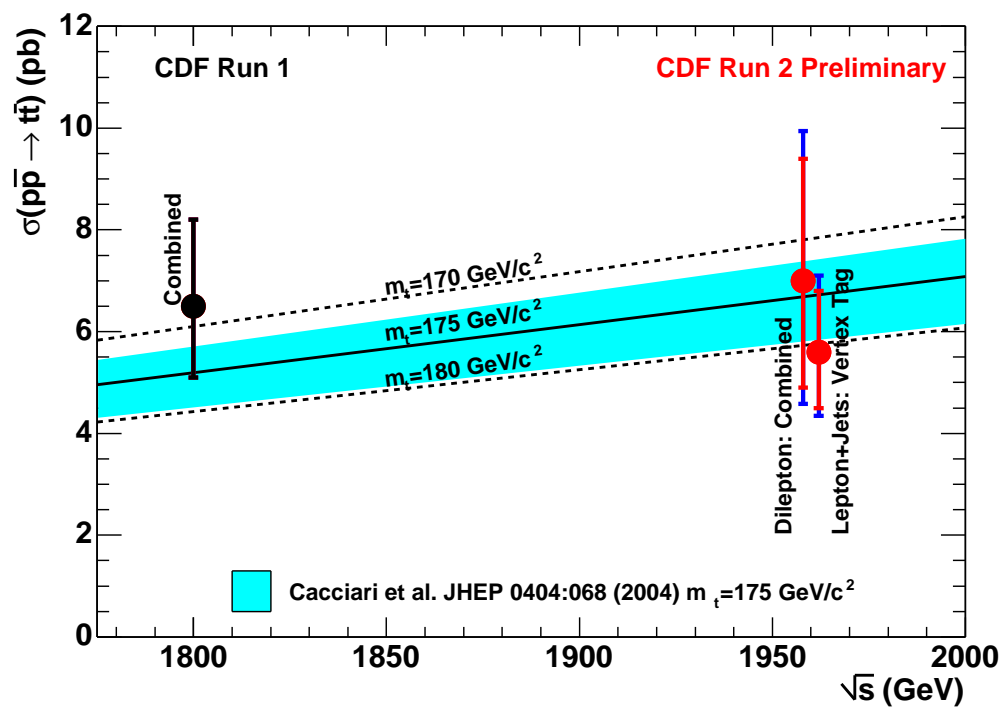

Figure 1.2: $t \bar{t}$ production cross section measured at CDF in Run I $(\sqrt{s}=1.8 \mathrm{TeV})$ and Run II $(\sqrt{s}=1.96 \mathrm{TeV})$. The band shows the theoretical prediction of the Standard Model.

Top quark has a very large mass width of $\Gamma=2.5 \mathrm{GeV}$, which indicates that the typical time scale of the decay is very short. In fact, top quark is expected to decay before it forms a hadron. In the Standard Model, top quark decays $100 \%$ of the time into a $W$ boson and a $b$ quark. Therefore, we categorize the $t \bar{t}$ production events into four categories, di-lepton, 
lepton+jets, all-hadronic and $\tau$ channels, due to the decay modes of the two $W$ bosons produced in the decays of top and anti-top quarks. Although the $\tau$ particle is a lepton, its identification requires a complicated analysis technique [9] due to its short lifetime and decay modes involving $\tau$ particles are categorized into an independent channel. Figure 1.3 is a diagram showing the lepton + jets decay of $t \bar{t}$ pair. Table 1.3 summarizes the categorization of the decay modes, with

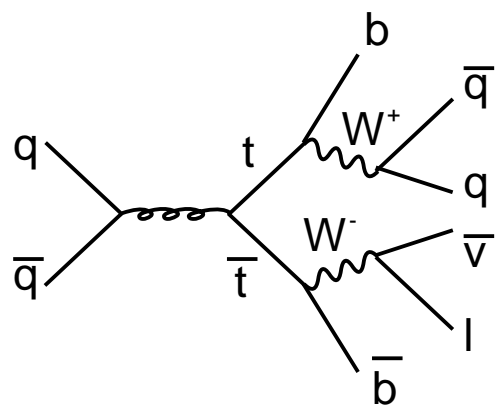

Figure 1.3: A diagram for $t \bar{t}$ production by $q \bar{q}$ annihilation and its decay into lepton + jets channel.

the branching ratio at the tree-level. The di-lepton channel is a clean channel in that it has

\begin{tabular}{lcc}
\hline \hline Decay mode & Branching Ratio & Channel Category \\
\hline$t \bar{t} \rightarrow\left(q \bar{q}^{\prime} b\right)\left(q \bar{q}^{\prime} \bar{b}\right)$ & $36 / 81$ & All-hadronic \\
$t \bar{t} \rightarrow\left(q \bar{q}^{\prime} b\right)(e \nu \bar{b})$ & $12 / 81$ & Lepton+jets \\
$t \bar{t} \rightarrow\left(q \bar{q}^{\prime} b\right)(\mu \nu \bar{b})$ & $12 / 81$ & Lepton+jets \\
$t \bar{t} \rightarrow\left(q \bar{q}^{\prime} b\right)(\tau \nu \bar{b})$ & $12 / 81$ & $\tau$ channel \\
$t \bar{t} \rightarrow(e \nu b)(\mu \nu \bar{b})$ & $2 / 81$ & Di-lepton \\
$t \bar{t} \rightarrow(e \nu b)(\tau \nu \bar{b})$ & $2 / 81$ & $\tau$ channel \\
$t \bar{t} \rightarrow(\mu \nu b)(\tau \nu \bar{b})$ & $2 / 81$ & $\tau$ channel \\
$t \bar{t} \rightarrow(e \nu b)(e \nu \bar{b})$ & $1 / 81$ & Di-lepton \\
$t \bar{t} \rightarrow(\mu \nu b)(\mu \nu \bar{b})$ & $1 / 81$ & Di-lepton \\
$t \bar{t} \rightarrow(\tau \nu b)(\tau \nu \bar{b})$ & $1 / 81$ & $\tau$ channel \\
\hline \hline
\end{tabular}

Table 1.3: Branching Ratios for $t \bar{t}$ decay modes in standard model coupling. $q$ stands for a $u, d, c$ or $s$ quark. Decay modes are categorized into four channels: All-jets, Lepton+jets, Di-lepton and $\tau$ channels.

few background sources. On the other hand, this channel has a small branching ratio. At the same time, the event reconstruction in this channel is challenging because two neutrinos, which can not be detected in the detector, are produced as the final particle in the $t \bar{t}$ decay chain. The lepton + jets channel is a channel with sufficiently large branching ratio of about $30 \%$ with 
reasonably well understood background. In fact, this channel showed the best sensitivity in the measurement of the mass of the top quark in Run I. The all-hadronic channel has a large branching ratio, with all the final particles detected as jets. However, the background rate for this channel is a huge one.

\section{The Mass of Top Quark}

The mass of the top quark was measured in Run I of CDF and D0 experiments at the centerof-mass energy of $1.8 \mathrm{TeV}$. Both collaborations carried out measurements in the di-lepton [10, 15] and the lepton + jets $[12,13]$ channels. CDF collaboration also reported a measurement result in the all-hadronic channel [14]. These measurements have been averaged to the top quark mass of $178.0 \pm 2.7 \pm 3.3 \mathrm{GeV} / \mathrm{c}^{2}[16]$. Figure 1.4 summarizes these Run I results.

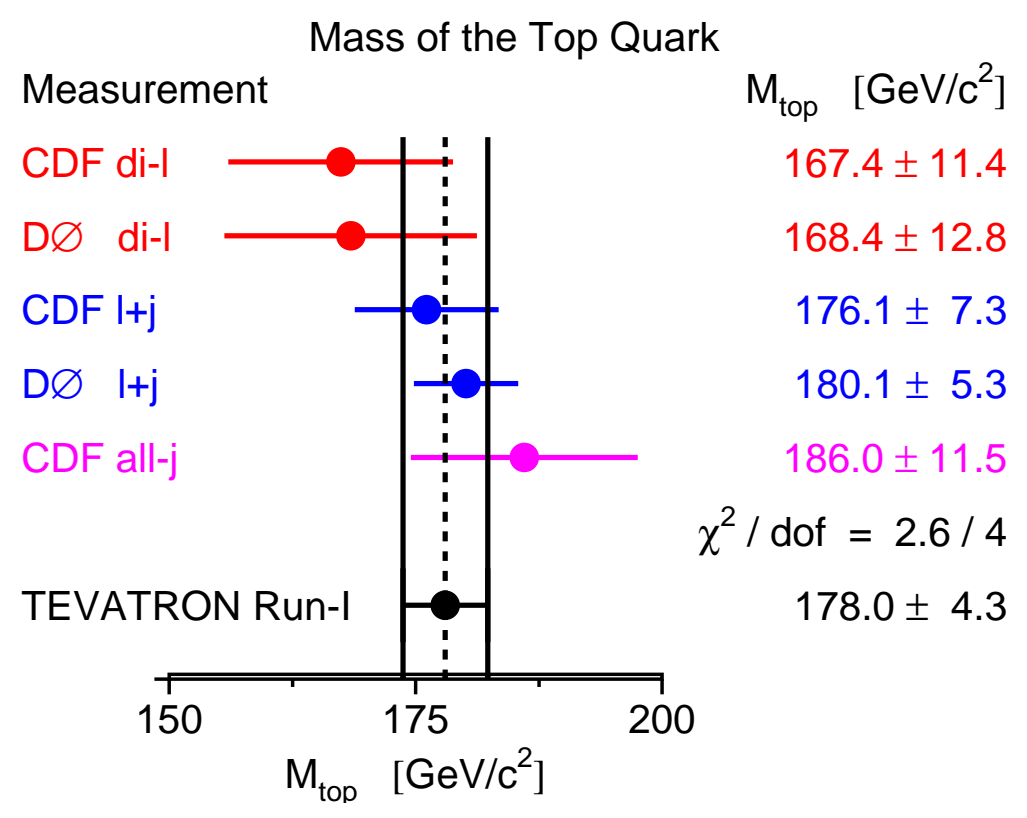

Figure 1.4: Summary of top quark mass measured by using Run I Tevatron data. di-l, $1+\mathrm{j}$ and all-j stand for dilepton, lepton+jets and all-hadronic channels respectively.

The top quark mass is one of the most desired quantities to be better measured in the particle physics. The Standard Model is not able to predict it, and it is a basic input to the Standard Model calculations. Furthermore, as illustrated below, a precise measurement of the mass of the top quark, along with that of the $W$ boson, provides a constraint on the mass of the Higgs boson. Such a constraint can bring us a hint in the search for the Higgs boson. At the tree level calculation of the Standard Model, there is an equation :

$$
M_{W}^{2}=\frac{\frac{\pi \alpha}{\sqrt{2} G_{F}}}{\sin ^{2} \theta_{W}}
$$


where $M_{W}, \alpha, G_{F}$ and $\theta_{W}$ are the mass of $W$ boson, the fine structure constant, the Fermi coupling constant and the electroweak mixing angle, respectively. At one loop calculation, this expression is modified :

$$
M_{W}^{2}=\frac{\frac{\pi \alpha}{\sqrt{2} G_{F}}}{\sin ^{2} \theta_{W}(1+\Delta r)}
$$

where $\Delta r$ contains the one-loop corrections [17]. The top quark makes a contribution to $\Delta r$ via the one loop diagrams shown in Figure 1.5, which contribute to the $W$ and $Z$ masses :

$$
(\Delta r)_{t o p} \approx-\frac{3 G_{F} m_{t}^{2}}{8 \sqrt{2} \pi^{2}} \frac{1}{\tan ^{2} \theta_{W}}
$$

The Higgs boson also contributes to $\Delta r$ via diagrams shown in Figure 1.6 :

$$
(\Delta r)_{H i g g s} \approx \frac{11 G_{F} M_{Z}^{2} \cos ^{2} \theta_{W}}{24 \sqrt{2} \pi^{2}} \ln \frac{m_{h}^{2}}{M_{Z}^{2}}
$$
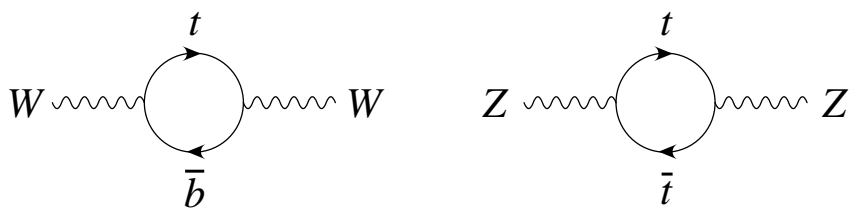

Figure 1.5: Virtual top quark loops contributing to $W$ and $Z$ masses.

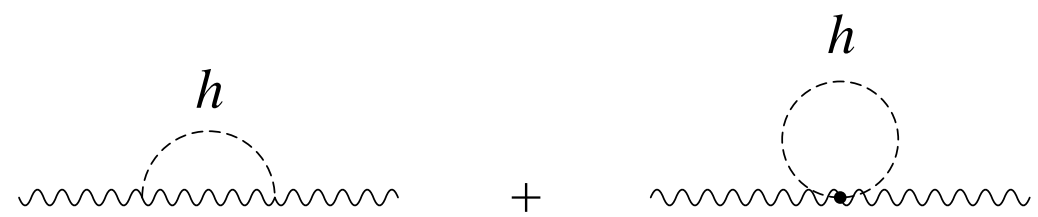

Figure 1.6: Virtual Higgs boson loops contributing to $W$ and $Z$ masses.

Figure 1.7 shows the current $68 \%$ confidence level contour for $M_{W}$ and $m_{\text {top }}$ for both indirect and direct measurements [18]. The indirect measurement was obtained using results from SLD and LEP collaborations. The direct measurement of $M_{W}$ is the average of LEP and Tevatron Run I, and the $m_{t o p}$ is the average of Tevatron Run I. The aimed for size of the uncertainty at $2 \mathrm{fb}^{-1}$ in Tevatron Run II is also shown. The Standard Model relationship for the masses as a function of the Higgs mass due to the one-loop corrections is also shown in the figure.

Figure 1.8 further shows the $\chi^{2}$ as a function of the Higgs mass obtained in a fit of the Standard Model to the electroweak measurements of LEP, SLD and Tevatron Run I collaborations, including the measurements of the top quark mass [18]. The $95 \%$ confidence level lower limit for the mass of Higgs boson obtained in direct search of $114.4 \mathrm{GeV} / c^{2}[19]$ is also shown. This plot 


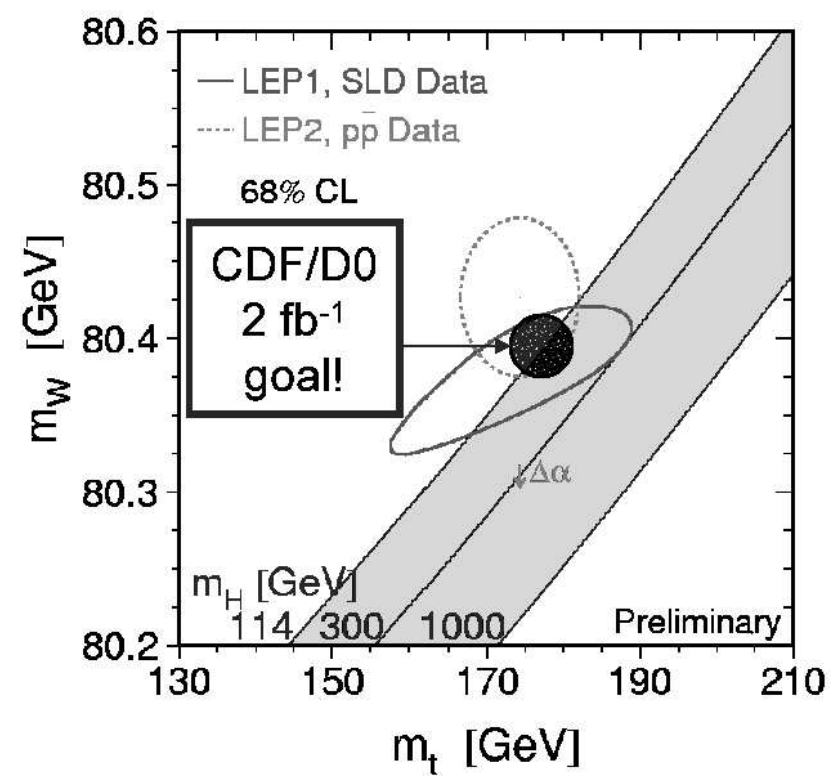

Figure 1.7: The $68 \%$ confidence level contour for $m_{W}$ and $m_{t o p}$. Contours for indirect and direct measurements are plotted as well as the aimed for contour of Tevatron Run II. Also shown is the Standard Model relationship for the masses as a function of the Higgs mass.

clearly shows that the electroweak measurements place a constraint to the mass of the Higgs boson. The $95 \%$ confidence level upper limit for the Higgs boson mass due to the $\chi^{2}$ curve is $260 \mathrm{GeV} / c^{2}[18]$.

The goal of this dissertation is to measure the mass of the top quark using $t \bar{t}$ pair creation events decaying into lepton + jets channel. We suppose the decay properties of the top quark as predicted by the Standard Model. For each candidate event found in data we calculate a "reconstructed mass" of the top quark. On the other hand, we parametrize the distributions of the reconstructed mass for signal and background events by using Monte Carlo samples. Especially for the signal process, the parametrization is done so that the distribution is described as a function of the mass of the top quark. The top quark mass is measured by an unbinned likelihood fit, where the top quark mass that describes the distribution extracted from data best, with the number of background events constrained around our estimation. The study presented in this dissertation used the data collected by the CDF detector (the Collider Detector at Fermilab) in $p \bar{p}$ collisions at the center-of-mass energy $\sqrt{s}=1.96 \mathrm{GeV}$ from March 2002 through August 2003 in Run II. We divide our event samples into three categories due to the number of $b$-tagged jets in the event : 0 tag, 1 tag and 2 tag samples. The integrated luminosities corresponding to the data used in this analysis are :

- $193 \mathrm{pb}^{-1}$ for 0 tag sample. 


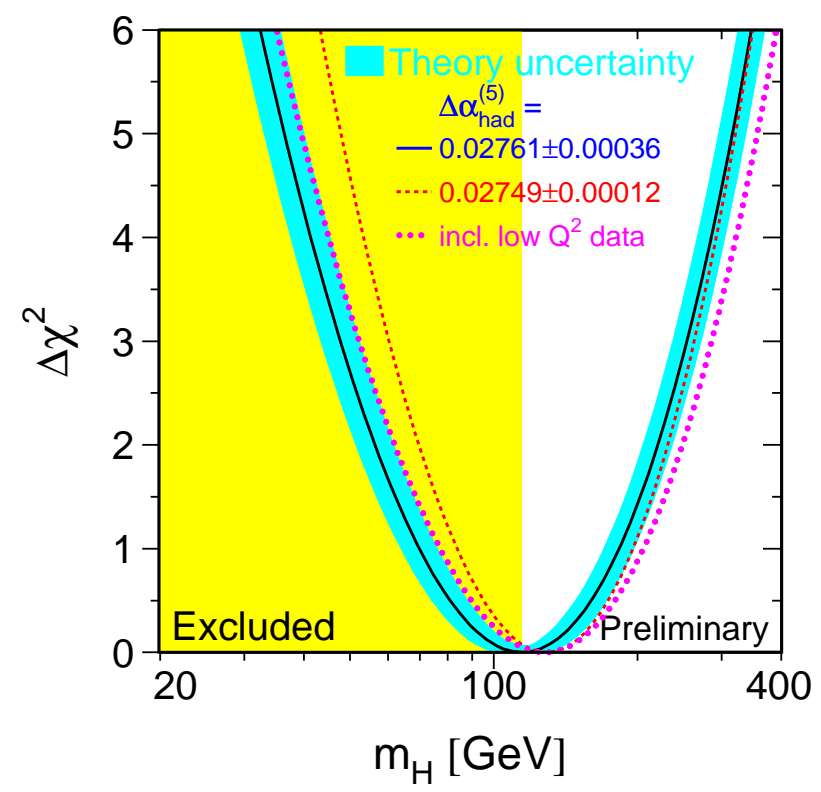

Figure 1.8: $\Delta \chi^{2}=\chi^{2}-\chi_{\text {min }}^{2}$ vs Higgs boson mass $M_{H}$. The line shows the best fit. The band represents an estimate of the theoretical error due to missing higher order corrections. The vertical band shows the $95 \%$ confidence level exclusion limit on $M_{H}$ from the direct search. The dashed curve is the result obtained using the evaluation of $\Delta \alpha_{h a d}^{(5)}\left(M_{Z}^{2}\right)$ from [20]

- $162 \mathrm{pb}^{-1}$ for 1 and 2 tag samples.

We will describe our experimental apparatus, Tevatron accelerator and CDF detector in Chapter 2. The method of particle identification and the measurement of physical variables concerning the particle is described in Chapter 3. Chapter 4 describes our data sample, Monte Carlo generators used in the analysis, and the event selection criteria. The definitions for our subsamples is also documented there. In Chapter 5, we illustrate the method with which we reconstructed each candidate event and extracted the reconstructed top mass. In Chapter 6, we parametrize the distributions of the reconstructed mass for signal and background processes. The number of background events is estimated in the course of making up the background distribution. The likelihood is defined in Chapter 7, and the method in which we measure the mass of the top quark using the Monte Carlo distributions is illustrated there. In Chapters 8 we perform pseudo-experiments in order to understand our statistical sensitivity for the mass of the top quark. At the same time, we optimize one of our b-tagging algorithms. In Chapter 9 , we again perform pseudo-experiments in order to check our method for biases. We estimate the systematic uncertainty of our top mass measurement in Chapter 10. Finally in Chapter 11, we look at our data sample and measure the mass of the top quark using the collision data of CDF. Chapter 12 summarizes the result of our measurement. 


\section{Chapter 2}

\section{Experimental Apparatus}

\subsection{The Accelerator Complex}

In the next decade, CDF plans to carry out precise analyses of several rare physical processes whose cross section is several orders of magnitude smaller than the inelastic $p \bar{p}$ cross section. In order to obtain sufficiently large samples, several steps have been taken:

- Increase the center-of-mass energy

- Increase the luminosity

- Increase the detector's acceptance

The first two steps, and the partial reconstruction of the Tevatron which they implied, are the topic of this section.

As was stated above, the Run II proton-antiproton center of mass energy has increased to $1.96 \mathrm{TeV}$ from the Run I value of $1.8 \mathrm{TeV}$. This change provides a major increase in the reconstructed sample size; for example, the cross section for associated $t \bar{t}$ production grows by $40 \%$ with respect to Run I.

Another way to obtain a larger sample is to increase the accelerator's luminosity. In the ideal case, where the proton and antiproton beams collide head-on without a crossing angle and with optimal alignment, the Tevatron's luminosity is given by the formula

$$
\mathcal{L}=\frac{f B N_{p} N_{\bar{p}}}{2 \pi\left(\sigma_{p}^{2}+\sigma_{\bar{p}}^{2}\right)} F\left(\frac{\sigma_{l}}{\beta^{*}}\right)
$$

where $f$ is the revolution frequency, B the number of bunches in each beam, $N_{p}$ and $N_{\bar{p}}$ the number of protons and anti-protons per bunch, $\sigma_{p}$ and $\sigma_{\bar{p}}$ the transverse beam sizes (RMS) 
at the interaction point, and $F$ a form factor that depends on the ratio between the bunch longitudinal RMS size, $\sigma_{l}$, and the beta function ${ }^{1}$ at the interaction point, $\beta^{*}$.

\begin{tabular}{|c|c|c|c|}
\hline \hline Run & 1989 & IA (1992-93) & IB (1993-95) \\
\hline$p /$ bunch & $7.00 \mathrm{E}+10$ & $1.20 \mathrm{E}+11$ & $2.32 \mathrm{E}+11$ \\
$\bar{p} /$ bunch & $2.90 \mathrm{E}+10$ & $3.10 \mathrm{E}+10$ & $5.50 \mathrm{E}+10$ \\
$p$ emittance (mm mrad) & 25 & 20 & 23 \\
$\bar{p}$ emittance (mm mrad) & 18 & 12 & 13 \\
Beta @ @ IP (m) & 0.55 & 0.35 & 0.35 \\
Energy (GeV/particle) & 900 & 900 & 900 \\
Bunches & 6 & 6 & 6 \\
Bunch length (rms, m) & 0.65 & 0.55 & 0.6 \\
Form Factor & 0.71 & 0.62 & 0.59 \\
Typical $\mathcal{L}\left(\mathrm{cm}^{-2} \mathrm{~s}^{-1}\right)$ & $1.60 \mathrm{E}+30$ & $5.42 \mathrm{E}+30$ & $1.58 \mathrm{E}+31$ \\
Best $\mathcal{L}\left(\mathrm{cm}^{-2} \mathrm{~s}^{-1}\right)$ & $2.05 \mathrm{E}+30$ & $9.22 \mathrm{E}+30$ & $2.50 \mathrm{E}+31$ \\
$\int \mathcal{L} d t$ (pb ${ }^{-1} /$ week) & 0.32 & 1.09 & 3.18 \\
Bunch Spacing (nsec) & 3500 & 3500 & 3500 \\
Interactions/crossing & 0.25 & 0.85 & 2.48 \\
What's New? & & Separators & Linac Upgrade \\
\hline \hline
\end{tabular}

Table 2.1: Evolution of Tevatron parameters. "Typical" luminosity is quoted at the beginning of a store.

As shown in Table 2.1 and Table 2.2, the most significant improvements in luminosity are obtained by increasing the number of bunches per beam from 6 to 36 , and then to 108, while keeping the number of particles per bunch similar to or higher than the Run I figure.

A limiting factor in the choice of accelerator parameters is the superposition of multiple elementary proton-antiproton interactions within the same bunch crossing. At high luminosities, this superposition increases the complexity of the event, making its reconstruction more difficult.

Production and acceleration of protons and anti-protons at Fermilab requires a chain of accelerators, each boosting particles to higher energies. Each step will be described in the following pages.

\footnotetext{
${ }^{1}$ Supposing the profile of the beam in the phase space $\left(x, x^{\prime}\right)$ is an ellipse of semi-axes $\sigma$ and $\sigma^{\prime}$, the amplitude function $\beta$ is defined as the ratio $\sigma / \sigma^{\prime}$, while the beam emittance is the phase volume $\epsilon=\pi \sigma \sigma^{\prime}$.
} 


\begin{tabular}{|c|c|}
\hline \hline Run & II (2001- ) \\
\hline$p /$ bunch & $3.30 \mathrm{E}+11$ \\
$\bar{p} /$ bunch & $3.60 \mathrm{E}+10$ \\
$p$ emittance (mm mrad) & 30 \\
$\bar{p}$ emittance (mm mrad) & 20 \\
Beta @@ IP (m) & 0.35 \\
Energy (GeV/particle) & 980 \\
Bunches & 36 \\
Bunch length (rms, m) & 0.43 \\
Form factor & 0.70 \\
Typical $\mathcal{L}$ (cm $\left.{ }^{-2} \mathrm{~s}^{-1}\right)$ & $4-10 \mathrm{E}+31$ \\
$\int \mathcal{L} d t$ (pb ${ }^{-1} /$ week) & 8 \\
Bunch Spacing (nsec) & 396 \\
Interactions/crossing & 2.17 \\
What's New? & Main Injector \\
$\bar{p}$ & $\bar{p}$ improvements \\
\hline \hline
\end{tabular}

Table 2.2: Evolution of Tevatron parameters. "Typical" luminosity is quoted at the beginning of a store. 


\subsubsection{Proton Production and Boosting}

The process begins with a Cockcroft-Walton accelerator, which feeds negative hydrogen ions to a $150 \mathrm{~m}$ linear accelerator. The Linac itself was upgraded in 1993, increasing its energy from $200 \mathrm{MeV}$ to $400 \mathrm{MeV}$; this made it possible, during Run $\mathrm{Ib}$, to double the number of protons per bunch, and to increase by about $50 \%$ the production rate of antiprotons.

After being stripped of electrons, the protons enter the Booster, a synchrotron whose diameter is about $150 \mathrm{~m}$, where they reach a kinetic energy of $8 \mathrm{GeV}$. Together, Linac and Booster are able to provide pulses of $5 \cdot 10^{12}$ protons for antiproton production every $1.5 \mathrm{~s}$, or $6 \cdot 10^{10}$ protons per bunch in series of 5 to 7 bunches, repeated 36 times every four seconds.

After leaving the Booster, protons are transferred to the Main Injector, a newly built circular accelerator that replaced the older Main Ring.

\subsubsection{Main Injector}

The Main Ring was originally built to provide $400 \mathrm{GeV}$ protons to Fermilab's fixed target experiments; later on, it was converted to act as an injector to the Tevatron. The new operational requirements for the Main Ring did not match its original design; therefore, during Run I, the Main Ring was a performance bottleneck. To quote an example, the Main Ring was never able to make full use of the Booster's capabilities: the Main Ring's aperture $(12 \pi \mathrm{mm} \mathrm{mrad})^{2}$ is only $60 \%$ of the Booster's aperture $(20 \pi \mathrm{mm}$ mrad). The situation would be even worse in Run II, with the Booster's aperture at injection increasing to $30 \pi \mathrm{mm}-\mathrm{mrad}$.

The Main Injector was designed to solve this problem, while providing further benefits. It is a 3-km circular accelerator, which brings protons and anti-protons from a kinetic energy of 8 $\mathrm{GeV}$ to a total energy of up to $150 \mathrm{GeV}$. Its transverse admittance is larger than $40 \pi \mathrm{mm} \mathrm{mrad}$, more than enough to accommodate particle bunches from the Booster; its emittance is about $12 \pi \mathrm{mm}$ mrad. The maximum beam size is $3 \cdot 10^{13}$ particles, divided into up to 504 bunches of $6 \cdot 10^{10}$ (anti)protons.

Being more flexible than the Main Ring, the Main Injector can be used in several operation modes:

- Antiproton production;

- Proton and antiproton boosting, before injection into the Tevatron in collider mode;

- Antiproton deceleration, in order to recover unused anti-protons after a Tevatron collision run;

- Proton and antiproton acceleration for fixed target experiments, either directly or as a booster for the Tevatron.

\footnotetext{
${ }^{2}$ All emittance are normalized at $95 \%$ of the beam.
} 


\subsubsection{Antiproton Production}

In order to produce anti-protons, a pulse of $5 \cdot 10^{12}$ protons at $120 \mathrm{GeV}$ is extracted from the Main Injector and focused on a nickel target. A lithium lens collects the anti-protons produced by the collision, with a wide acceptance around the forward direction, at energies close to $8 \mathrm{GeV}$. The antiproton bunches are then moved to a Debuncher Ring, where they are transformed into a continuous beam and stochastically cooled, and then to the Accumulator, where they are further cooled. The antiproton stacking rate during Run I was about $7 \cdot 10^{10} \bar{p}$ /hour; Run II upgrades, ranging from antiproton cooling to improving the lithium lens, increases this rate by a factor of three to four.

When a sufficient number of anti-protons (up to $10^{12}$ ) is available, stacking is suspended; the anti-protons are further cooled, and then transferred, with an aperture of $10 \pi \mathrm{mm} \mathrm{mrad}$ and a $\Delta p / p<10^{-3}$, to the antiproton Recycler Ring.

\subsubsection{Recycler Ring}

The Recycler Ring lies in the same enclosure as the Main Injector; contrarily to the other rings at Fermilab, it is built with permanent magnets. During Run I, the antiproton accumulation ring was found to suffer some kind of failure approximately once a week; this led to the loss of the entire store. Permanent magnets, not being prone to the most common causes of failure (such as power loss and lightning) provide a very stable repository for up to $3 \cdot 10^{12}$ anti-protons at a time.

During Run II, bunches of $2 \cdot 10^{11}$ recently produced anti-protons are transferred from the Accumulator to the Recycler Ring every about half an hour, thus keeping the total beam current in the Accumulator small (below $10 \mathrm{~mA}$, compared to the $200 \mathrm{~mA}$ antiproton current in Run I).

Antiproton production is one of the limiting factors in the efficiency of Fermilab's colliders. At the end of a store, $75 \%$ of the antiprotons are expected to be still circulating in the Tevatron; by recycling $2 / 3$ of these anti-protons, the average luminosity can be increased by a factor of two.

\subsubsection{Tevatron}

The Tevatron is about 6 -km circular accelerator, where protons and anti-protons, rotating in opposite directions inside the same beam pipe, are accelerated from $150 \mathrm{GeV}$ to $1 \mathrm{TeV}$. Making use of the upgrades in the rest of the accelerator chain, the Tevatron can provide an initial luminosity of $5 \cdot 10^{31} \mathrm{~cm}^{2} \mathrm{~s}^{-1}$.

During a collider store, instant luminosity slowly decreases. In the early stages of the store, the most important cause for this decrease is intrabeam scattering; some hours later, the depletion of anti-protons during collisions becomes more relevant. Luminosity is expected to decrease to $50 \%$ in about seven hours, and to $1 / e$ in twelve hours. After a typical store duration of eight 
hours, $75 \%$ of the antiprotons are still available; they are decelerated in the Tevatron and in the Main Injector, and then stored in the Recycler Ring and re-cooled Recycler is not used for the current $\bar{p} p$ collisions.

The Tevatron can also be used in fixed-target mode: it can accelerate up to $3 \cdot 10^{13}$ protons at a time to an energy of $800 \mathrm{GeV}$, and deliver single bunches to be used in proton, meson and neutrino experiments.

Other operational parameters of the Tevatron are listed in Table 2.1 and Table 2.2.

\subsubsection{Beam Monitors}

Operation of colliders at the Tevatron requires a constant monitoring of the beam position and luminosity. From a conceptual point of view, this is done in Run II as it was done in Run I.

The luminosity monitor consists in two arrays of scintillators, placed on both sides of the interaction region. A coincidence of particles moving away from the interaction point, both in the $p$ and $\bar{p}$ direction, is interpreted as a contribution to luminosity; bunches of particles moving in a single direction, without a coincident bunch in the opposite direction, are flagged as beam losses.

The beam position, on the other hand, is measured by the collider detectors themselves. During Run I, the detector was able to locate the beam within $5 \mu \mathrm{m}$ in about five minutes; other beam parameters, such as slope and transverse profile, were calculated over longer time intervals (about two hours). In Run II, the same operations are performed more quickly.

\subsection{The CDF Detector}

As stated above, one of the aims of Run II is to reconstruct and store a large sample of rare events. To achieve this result, the number of bunches in each beam increased first by a factor of six with respect to Run I. An immediate consequence is that the time between two successive interactions decreased by the same factor. Several parts of the detectors have been rebuilt from scratch in order to accommodate the higher collision rate.

While the detector was redesigned, efforts were also made to extend its acceptance. The geometrical coverage was increased, by adding new detector elements or enlarging the previously existing ones; the trigger system became able to detect some interesting event features at an earlier stage than in Run I, thus improving the signal to background ratio.

As shown in figure 2.1, the tracking system of CDF II is placed inside a superconducting solenoid, while calorimeter and muon systems are outside the magnet. The rest of this chapter will provide a short description of the detector subsystems, with an emphasis on the upgrades since Run I. A complete description of CDF can be found in [21].

In the standard CDF geometry, the $\hat{z}$ axis is oriented along the axis of the solenoid, the $\hat{x}$ axis points away from the center of the Tevatron, and the $\hat{y}$ axis points up. The origin is at the 
interaction point. The polar angle $\theta$ is measured starting from the positive $\hat{z}$ axis; the rapidity $y$ is defined by

$$
y=\frac{1}{2} \ln \left(\frac{E+p_{z}}{E-p_{z}}\right)
$$

For the high energy particles, $E \sim p$ and $p_{z}=p \cos \theta$, hence the pseudo-rapidity is defined as

$$
\eta=-\ln \left(\tan \frac{\theta}{2}\right)
$$

In hadron-hadron collisions, a rapidity $y$ (or pseudo-rapidity $\eta$ ), a transverse momentum $p_{T}$ and an azimuth angle $\phi$ are usually used. The invariant cross section is written as

$$
E \frac{d^{3} \sigma}{d^{3} p}=\frac{d^{3} \sigma}{d \phi d y p_{T} d p_{T}} \rightarrow \frac{d^{3} \sigma}{\pi d y d p_{T}^{2}}
$$

The second form is obtained using the identity $d y / p_{z}=1 / E$, and the third form represents the average over $\phi$. The total multiplicity of particles in collisions is given by $d \sigma / d y$ and this means that the multiplicity is flat in $\eta$.

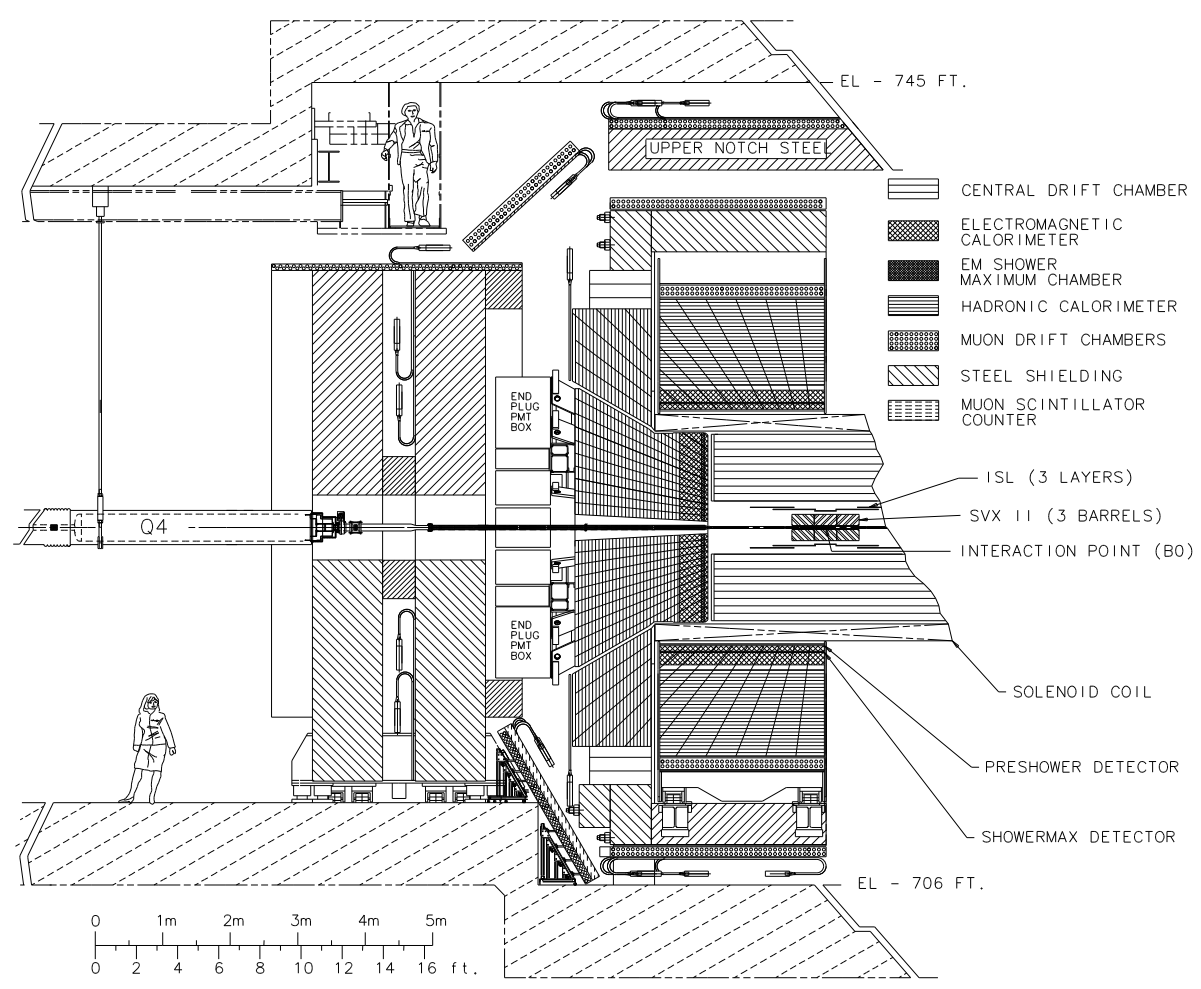

Figure 2.1: Elevation view of one half of the CDF II detector 


\subsection{Tracking System}

The innermost parts of the CDF II detector are devoted to tracking charged particles.

\subsubsection{Silicon Vertex Detector}

CDF II makes use of three concentric silicon detectors: "Layer 00" (L00), the Silicon Vertex Detector (SVX II, or SVX in short), and the Intermediate Silicon Layers (ISL) [22].

SVX II is the Run II baseline detector. It consists of five layers of double-sided silicon wafers. One side of each wafer provides measurements in the transverse plane (axial strips); the other side's strips deliver 3D information. SVX II extends radially from 2.5 to $10 \mathrm{~cm}$, and along $z$ up to $45 \mathrm{~cm}$ on either side of the interaction point.

The ISL consists of a double-sided silicon layer, similar to those in SVX II, placed at $r=$ $22 \mathrm{~cm}$ in the central $\eta$ region, and of two forward layers $(1<|\eta|<2)$ respectively at 20 and $29 \mathrm{~cm}$ from the beam line. Together with SVX II, the ISL makes it possible to reconstruct tracks in the forward region, which lies beyond the acceptance region of the outer tracker.

Layer 00 is the most recent addition to the CDF II tracker. It is a single sided, radiationhard silicon layer, placed immediately outside the beam pipe, at $r \simeq 1.5 \mathrm{~cm}$. Being so close to the interaction point, Layer 00 improves noticeably the impact parameter resolution. In case the innermost SVX II layer suffers from radiation damage during Run II, Layer 00 also acts as a backup.

Compared to the shorter, 4-layer, single-sided vertex detector of Run I, the new silicon tracker provides a much wider acceptance, better resolution, three-dimensional reconstruction, and can be used in stand-alone mode, without input from the Central Outer Tracker (described hereafter).

\subsubsection{Central Outer Tracker}

Outside the silicon detector, at a distance between 40 and $138 \mathrm{~cm}$ from the beam, lies the Central Outer Tracker [23]. It is a new open-cell drift chamber, able to reconstruct tracks in the $|\eta|<1$ region. The COT replaces an older drift chamber, the CTC [24], that would have been unable to cope with the expected occupancy and event rate of Run II.

Each of the eight superlayers of cells consists of twelve layers of sense wires, alternating with field-shaping wires. Axial superlayers alternate with stereo superlayers, thus providing 48 axial and 48 stereo measurements for each track.

In the COT, the cell size is roughly four times smaller than in the CTC. Usage of a faster gas ( $\mathrm{Ar}-$ Ethane $-\mathrm{CF}_{4}$ instead of $\mathrm{Ar}-$ Ethane) reduces the maximum drift time by a further factor of two, down to $100 \mathrm{~ns}$. This makes the COT immune from event pile-up, even at the highest collision rate of $1 /(132 \mathrm{~ns})$. 


\subsubsection{Time of flight}

A recent addition to CDF II, the time-of-flight detector is an array of scintillator bars, placed at the outer edge of the COT, at a radial coordinate of $140 \mathrm{~cm}$. An accurate measurement of a particle's time of flight in the CDF tracking volume can be used quite effectively in particle identification.

Scintillator bars are about three meters long, matching the COT active volume; their thickness $(4 \mathrm{~cm})$ is limited by the space which remained available between the previously designed COT and magnet. Their width was determined by occupancy ${ }^{3}$ and resolution considerations; the best choice turned out to be also of the order of $4 \mathrm{~cm}$. The bars have a trapezoidal cross section, in order to minimize cracks in the geometry; the scintillating material is Bicron 408, which has a short rise time and a long $(380 \mathrm{~cm})$ attenuation length.

Photomultiplier tubes, attached to both ends of each bar, provide time and pulse height measurements. By comparing the two pairs of results, the detector determines the instant in which a particle crossed the scintillator with an accuracy of about $100 \mathrm{ps}$, and the $z$ coordinate of the intersection. The latter measurement is compared to the results of $3 \mathrm{D}$ track reconstruction in the inner tracking volume, to associate a time of flight to each track.

\subsubsection{Magnet}

The CDF tracking systems are enclosed in a superconducting solenoid, which provides a uniform magnetic field of up to $1.5 \mathrm{~T}$ along the detector axis, over a cylindrical fiducial volume $3.5 \mathrm{~m}$ long and $2.8 \mathrm{~m}$ in diameter.

The solenoid is built of an Al-stabilized NbTi superconductor, able to withstand currents up to $5000 \mathrm{~A}$, and operating at liquid helium temperature. During most of Run I, the magnet operated at $4650 \mathrm{~A}$, corresponding to a current density of $1115 \mathrm{~A} / \mathrm{m}$ and a central field of $1.41 \mathrm{~T}$.

Although the design lifetime of the solenoid was only ten years, it is possible to reuse the magnet during Run II. The cool-down procedures that were used during Run I limited mechanical stress to the coil, avoiding fatigue damage.

\subsection{Calorimetry}

\subsubsection{Overview}

CDF uses scintillator sampling calorimeters, divided into separate electromagnetic and hadronic sections, and providing coverage for $|\eta| \leq 3.64$. The calorimeter was an essential tool in selection and reconstruction of events in Run I; in Run II it continues to measure the energy of photons,

\footnotetext{
${ }^{3}$ Detector occupancy depends on the average number of superimposed interactions, which increases with luminosity. TOF occupancy is estimated to be 0.1 with 2 superimposed events, and 0.4 with 10 events.
} 
electrons, jets, and the missing transverse energy ${ }^{4}$ associated to neutrinos and possibly to neutral exotic particles.

Calorimeter calibration can be performed by matching the tracks found in the tracking system to the corresponding calorimetry towers; during Run I, this provided a $2.5 \%$ accuracy on jet energy measurements.

\begin{tabular}{c|c|c}
\hline \hline$|\eta|$ range & $\Delta \phi$ & $\Delta \eta$ \\
\hline $0-1.1(1.2 \mathrm{had})$ & $15^{\circ}$ & 0.1 \\
$1.1(1.2 \mathrm{had})-1.8$ & $7.5^{\circ}$ & 0.1 \\
$1.8-2.1$ & $7.5^{\circ}$ & 0.16 \\
$2.1-3.64$ & $15^{\circ}$ & $0.2-0.6$ \\
\hline \hline
\end{tabular}

Table 2.3: Calorimeter segmentation

The entire calorimeter is segmented into projective towers, whose geometry is summarized in table 2.3. Each tower consists of alternating layers of passive material (lead for the e.m. section, iron for the hadronic compartment) and scintillator tiles. The signal is read via wavelength shifters (WLS) embedded in the scintillator; light from the WLS is then carried to photomultiplier tubes. Table 2.4 shows the most important characteristics of each calorimeter sector. The central and end-wall calorimeters $(|\eta|<1.1)[25][26]$ were recycled from Run I; the plug ones $(1.1<|\eta|<3.64)$ were built anew, to replace an older gas calorimeter that would not be able to function at the increased event rate of Run II.

\subsubsection{Central Calorimeter}

Apart from the electronics, the central calorimeter in CDF Run II is the same used during Run I. The energy measurement response time is already fast enough to accommodate a $132 \mathrm{~ns}$ bunch spacing.

\section{Central Electromagnetic Calorimeter}

The central electromagnetic calorimeter consists of projective towers of alternating lead and scintillator. The signal is read via a $\mathrm{PMMA}^{5}$ wavelength shifter, and carried via clear fiber to photomultiplier tubes. None of these is expected to suffer much from radiation damage. The light yield loss is expected to be around $1 \%$ per year; $60 \%$ of this loss is explained by the gradual shortening of the attenuation length in the scintillator.

A two-dimensional wire chamber is embedded in the calorimeter, as a shower maximum detector (CES). Its usage in the Run I trigger decreased the fake electron trigger rate by a

\footnotetext{
${ }^{4}$ Contrarily to $e^{+} e^{-}$colliders, in $p \bar{p}$ colliders the longitudinal momentum of the initial state is unknown.

${ }^{5} \mathrm{PMMA}=$ polymethylmethacrylate
} 


\begin{tabular}{|c|c|c|}
\hline \hline & Central and End-wall & Plug \\
\hline Electromagnetic: & & \\
Thickness & $19 X_{0}, 1 \lambda$ & $21 X_{0}, 1 \lambda$ \\
- per sample (Pb) & $0.6 X_{0}$ & $0.8 X_{0}$ \\
- per sample (scint.) & $5 \mathrm{~mm}$ & $4.5 \mathrm{~mm}$ \\
Light yield & $160 \mathrm{p.e.} / \mathrm{GeV}$ & $300 \mathrm{p.e.} / \mathrm{GeV}$ \\
Sampling resolution & $11.6 \% / \sqrt{E}$ & $14 \% / \sqrt{E}$ \\
Stochastic resolution & $14 \% / \sqrt{E}$ & $16 \% / \sqrt{E}$ \\
\hline Hadronic: & & \\
Thickness & $4.5 \lambda$ & $7 \lambda$ \\
- per sample (Fe) & $1 \mathrm{in}(\mathrm{central})$ & $2 \mathrm{in}$ \\
- per sample (scint.) & $6 \mathrm{in}(\mathrm{end}-\mathrm{wall})$ & $6 \mathrm{~mm}$ \\
Light yield & $40 \mathrm{p.e.} / \mathrm{GeV}$ & $39 \mathrm{p.e} . / \mathrm{GeV}$ \\
Resolution & $75 \% / \sqrt{E} \oplus 3 \%$ & $80 \% / \sqrt{E} \oplus 5 \%$ \\
\hline \hline
\end{tabular}

Table 2.4: Characteristics of the CDF II calorimeter

factor of two [27].

Another wire chamber is placed immediately in front of the calorimeter, to act as a preshower detector (CPR) which uses the tracker and the solenoid coil as radiators. The CPR has proven to be extremely useful in rejection of electron background; it also reduced systematic uncertainties for direct photon measurements by a factor of three [28].

\section{Central Hadronic Calorimeter}

The central and end-wall hadronic calorimeters use 23 iron layers as radiator. The scintillator should not suffer radiation damage from measured events.

The hadronic compartment geometry matches the projective towers of the electromagnetic calorimeter.

\subsubsection{Plug Calorimeter Upgrade}

The CDF II plug calorimeter, shown in figure 2.2, covers the $\eta$ region between 1.1 and 3.64, corresponding to polar angles between $37^{\circ}$ and $3^{\circ}$. It replaces an older gas calorimeter, whose response speed was too slow for usage at the CDF II 132 ns inter-bunch. Being based on the same principles as the central calorimeter, the new plug calorimeter also makes experimental data more homogeneous. 


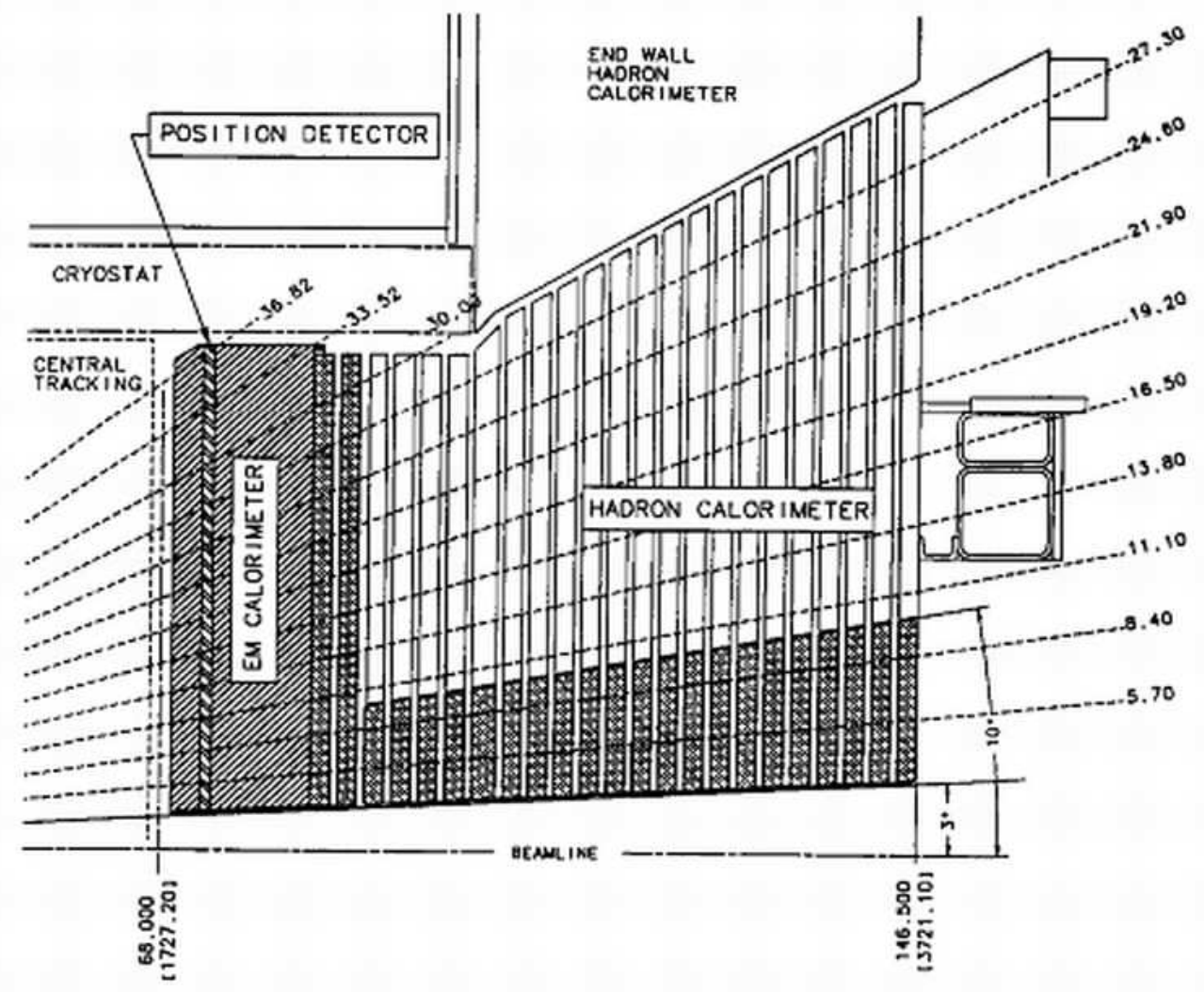

Figure 2.2: Cross section of half CDF II plug calorimeter 
The calorimeter is divided in 12 concentric $\eta$ regions, which are further segmented in 24 (for $|\eta|<2.11$ ) or 12 (for $|\eta|>2.11$ ) projective towers.

\section{Plug Electromagnetic Calorimeter}

The EM section of the plug calorimeter consists of 23 absorber-scintillator layers. A calciumtin-lead alloy, enclosed between steel plates, is used as absorber.

The first layer of the EM section is used as a preshower detector. In order to distinguish $\gamma$ from $\pi^{0}$ reliably, the light yield needs to be higher than on other layers. Therefore, the first scintillator layer is thicker $(10 \mathrm{~mm}$ instead of $6 \mathrm{~mm}$ ) and made of a brighter material; it is read out separately from the rest of the calorimeter, via multi-anode photomultiplier tubes (MAPMT).

As in the central calorimeter, a shower maximum detector (PES) is also embedded in the plug EM calorimeter, at a depth of about six radiation lengths. The PES consists of eight $45^{\circ}$ sectors, each covering six (or three) calorimetric towers in $\phi$; each sector is further segmented in two $\eta$ regions, in order to reduce occupancy. Within each region, scintillating strips are arranged on two layers, in directions parallel to either edge of the sector; this provides a two-dimensional measurement of the shower. The strips are $5 \mathrm{~mm}$ wide and $6 \mathrm{~mm}$ thick; they are read out via WLS fibers and MAPMT.

The PES is used to measure the position of electromagnetic showers with an accuracy reaching $1 \mathrm{~mm}$ for high-energy electrons, and to discriminate pions from photons and electrons.

\section{Plug Hadronic Calorimeter}

The hadron plug calorimeter was designed to optimize detector performance on $b$, electroweak and jet physics, and to help in muon detection by analyzing their rate of energy loss. It achieves an energy resolution of about $80 \% / \sqrt{E} \oplus 5 \%$, which is dominated by the sampling fluctuations from the steel absorber plates. The most strict requirement is that the light yield within each tile should be uniform to $4 \%$ or better; dis-uniformity between different tiles is not as important, as the hadron shower usually affects ten or more layers.

\subsection{Muon Chambers}

The outermost component of CDF II is a set of scintillators, drift tubes and steel absorbers, used for the detection of muons.

During Run I, detection of muons has proven to be an important requirement, both for the analysis of several physics channels and for calibration. For example, a clean sample of $W$ bosons is obtained by reconstructing their muon decay mode; $J / \psi \rightarrow \mu^{+} \mu^{-}$decays are an important part of the heavy quark physics program, as well as a tool to measure systematic effects in the detector. 
The tracking improvements from Run I to Run II have a deep impact on muon detection. Before the upgrades, muons in the central region were identified by their penetrating power, and their momentum was measured in the central tracking chamber. On the contrary, the momentum of forward muons had to be measured in the muon chambers themselves, by resorting to a toroidal magnet, as the central tracker only covered the $|\eta|<1$ region.

With the SVX II upgrade, this distinction falls: measurement of muon momentum can be performed in the central tracker, where the multiple scattering effects are smaller, and the toroidal magnets are not required any longer. Central tracks are measured in the drift chamber; forward tracks $(|\eta|>1)$ are tracked in the silicon only.

Run I central muon chambers (CMU) are reused without major changes; some upgrades which started under Run I (CMP and CSP, the Central Muon/Scintillator Upgrades; CMX and CSX, the Central Muon/Scintillator Extension) are completed; and a new set of chambers, the Intermediate Muon Detector IMU, replaces the previous Forward Muon Detectors (FMU)[29].

\begin{tabular}{lcccc}
\hline \hline & CMU & CMP/CSP & CMX/CSX & IMU \\
\hline$\eta$ coverage & $0-0.6$ & $0-0.6$ & $0.6-1.0$ & $1.0-1.5$ \\
\hline Drift tubes: & & & & \\
thickness & $2.68 \mathrm{~cm}$ & $2.5 \mathrm{~cm}$ & $2.5 \mathrm{~cm}$ & $2.5 \mathrm{~cm}$ \\
width & $6.35 \mathrm{~cm}$ & $15 \mathrm{~cm}$ & $15 \mathrm{~cm}$ & $8.4 \mathrm{~cm}$ \\
length & $226 \mathrm{~cm}$ & $640 \mathrm{~cm}$ & $180 \mathrm{~cm}$ & $363 \mathrm{~cm}$ \\
max drift time & $0.8 \mu \mathrm{s}$ & $1.4 \mu \mathrm{s}$ & $1.4 \mu \mathrm{s}$ & $0.8 \mu \mathrm{s}$ \\
$\#$ tubes (Run Ib) & 2304 & 864 & 1536 & - \\
$\#$ tubes (Run II) & 2304 & 1076 & 2208 & 1728 \\
\hline Scintillators: & & & & \\
thickness & N/A & $2.5 \mathrm{~cm}$ & $1.5 \mathrm{~cm}$ & $2.5 \mathrm{~cm}$ \\
width & N/A & $30 \mathrm{~cm}$ & $30-40 \mathrm{~cm}$ & $17 \mathrm{~cm}$ \\
length & N/A & $320 \mathrm{~cm}$ & $180 \mathrm{~cm}$ & $180 \mathrm{~cm}$ \\
$\#$ counters (Run Ib) & N/A & 128 & 256 & - \\
$\#$ counters (Run II) & N/A & 269 & 324 & 864 \\
\hline$\pi^{0}$ int. lengths & 5.5 & 7.8 & 6.2 & $6.2-20$ \\
Min $P_{t}$ (GeV/c) & 1.4 & 2.2 & 1.4 & $1.4-2.0$ \\
MS resol. (cm GeV) & 12 & 15 & 13 & $13-25$ \\
\hline \hline
\end{tabular}

Table 2.5: Parameters of muon detection at CDF. Pion interaction length and the limit on resolution due to multiple scattering are computed at $\theta=90^{\circ}$ in the central detectors $\mathrm{CMU}$, CMP and CSP; at $\theta=55^{\circ}$ in CMX and CSX; and on the entire $\theta$ coverage for the IMU.

Due to their size, muon systems are unable to take data within the Run II inter-bunch 
interval of 400 or $132 \mathrm{~ns}$; this is not a problem, since the low occupancy of the muon chambers allows integration over multiple events. Scintillators are used to associate muon stubs to the appropriate event.

Table 2.5 summarizes the information on the muon subsystems; the following sections will describe their characteristics in deeper detail.
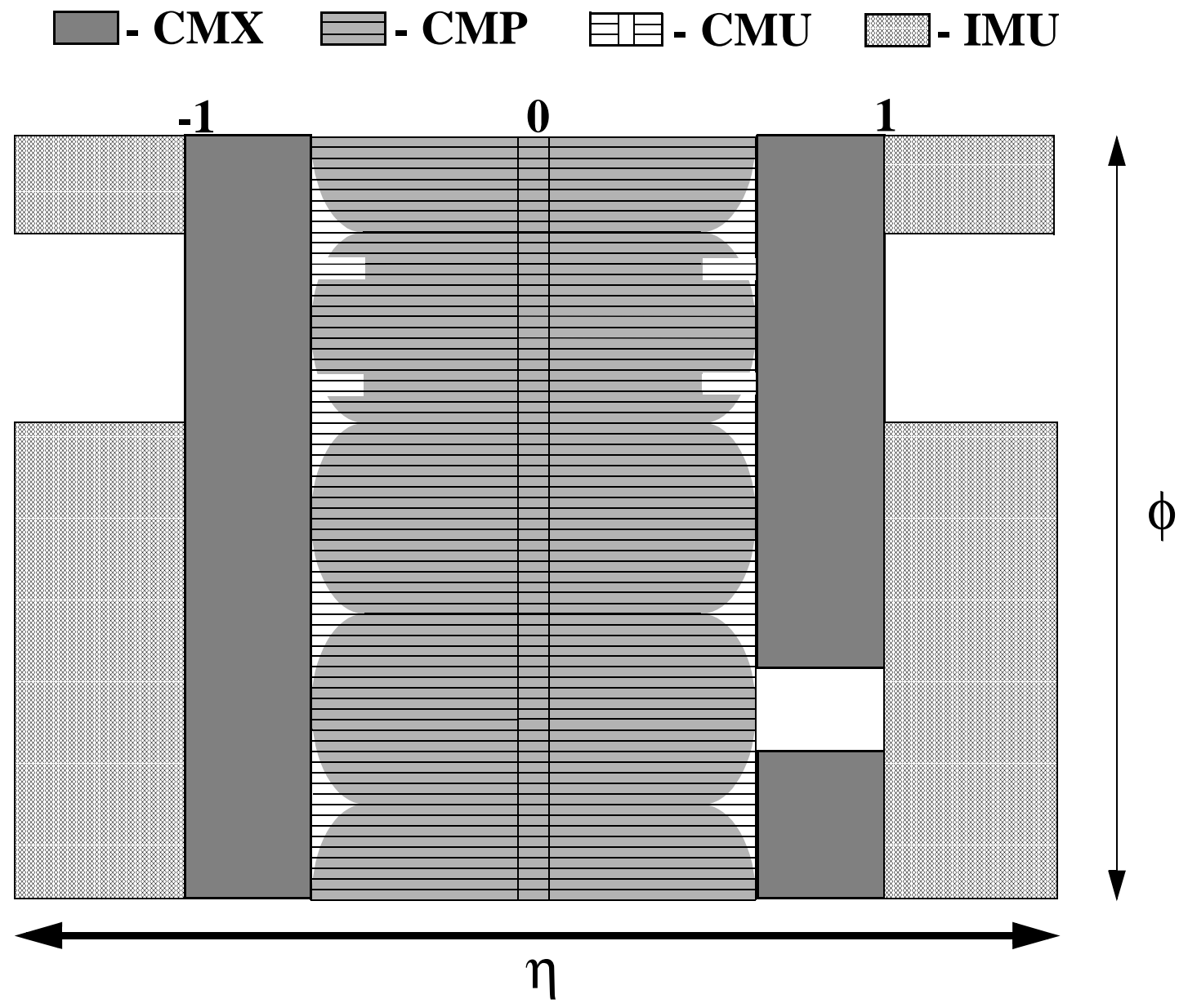

Figure 2.3: $\eta$ and $\phi$ coverage of the Run II muon systems

\subsubsection{Central Muon Detectors}

The first muon system built at CDF, the Central Muon Detector (CMU) [30], is a set of 144 modules, each containing four layers of four rectangular cells. It is placed just outside the central hadronic calorimeter, whose 5.5 interaction lengths absorb more than $99 \%$ of the outgoing charged hadrons. 
A second set of muon chambers, the Central Muon Upgrade (CMP) [31], forms a square box around the CMU, and is shielded by an additional layer of $60 \mathrm{~cm}$ of steel. Due to the detector geometry, the $\eta$ coverage varies with azimuth as shown in figure 2.3. The CMP consists of four layers of single-wire drift tubes, staggered by half cell per layer, and operated in proportional mode. On the outer surface of the CMP lies the Central Scintillator Upgrade (CSP), a layer of rectangular scintillator tiles.

Another upgrade which was begun in Run I is the Central Muon Extension (CMX) with the associated Central Scintillator Extension (CSX). It is a conical array of drift tubes, with scintillators on both sides; it extends the CMU/CMP $\theta$ coverage from $55^{\circ}$ to $42^{\circ}$, except in a $30^{\circ} \phi$ gap which is used by the solenoid cryogenic system.

\subsubsection{Intermediate Muon Detectors}

Detection of muons in the forward region is accomplished by the Intermediate Muon Detectors (IMU). This detector recycles the older Forward Muon toroidal magnets, which is moved closer to the interaction point (just outside the plug calorimeter PMT arrays). The steel toroids, together with a new pair of steel rings, act as shielding for a new array of drift tubes and scintillator counters, placed on the outer radius of the toroids.

Like the CMX/CSX, the IMU has four staggered layers of drift tubes, and two layers of scintillator. Contrarily to the CSX, one of the scintillator layers is separated from the drift tubes by a thick layer of steel; this geometry strongly suppresses fake triggers due to hadrons.

\subsection{Data Acquisition and Trigger}

Due to the increase in collision frequency, the DAQ and trigger systems of CDF had to be almost completely replaced. The new three-level architecture, schematized in figure 2.4 , is fully capable of withstanding a $132 \mathrm{~ns}$ bunch separation, while keeping dead time as short as possible.

\subsubsection{Level 1 trigger}

The front-end electronics of all detectors is fitted with a synchronous pipeline, 42 events deep, where the entire data regarding each event is stored for 5544 ns. Meanwhile, part of the data is examined in a first layer of dedicated, synchronous, highly parallel hardware processors:

- XFT, the extremely Fast Tracker, which reconstructs tracks on the transverse plane of the COT to propagate these tracks to the calorimeters and muon chambers;

- the Calorimeter Trigger, which detects electron and photon candidates, jets, total transverse energy, and missing transverse energy;

- the Muon Trigger, which matches XTRP tracks to stubs in the muon chambers. 


\section{Dataflow of CDF "Deadtimeless" Trigger and DAQ}

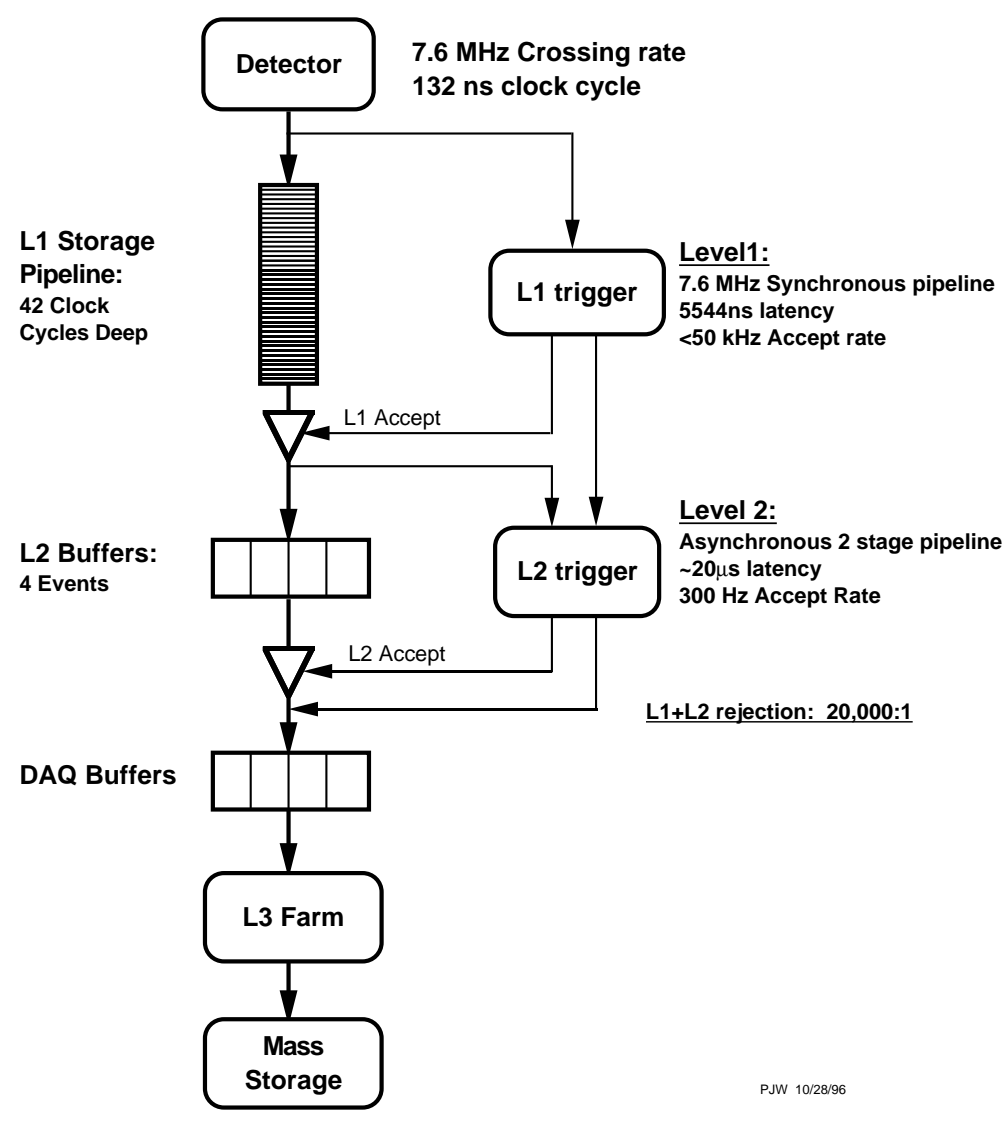

Figure 2.4: Block diagram of the CDF II Trigger 
"Objects" from the level one trigger subsystems are combined in a flexible decision module, which takes a decision by requiring the presence of a certain number of features in the event: for example, two muon candidates with $P_{t}$ above $3 \mathrm{GeV}$. Up to 64 different sets of requirements can be checked at the same time; each of these triggers can be pre-scaled independently of the others.

The level 1 trigger takes a decision within $4 \mu \mathrm{s}$, while the event's data is still in the pipeline. This makes the first trigger level truly dead-timeless. The rejection factor is about 150 and the event rate is about $50 \mathrm{kHz}$.

\subsubsection{Level 2 trigger}

Events matching the requirements of level 1 are downloaded into one of four asynchronous event buffers, and further analyzed by a second set of hardware processors. Trigger level 2 is asynchronous: events remain in the buffer until they are accepted or rejected. This can cause dead time, when all four buffers are full. In order to keep dead time at 10\%, with a level 1 rate of $50 \mathrm{kHz}$, level 2 has been split in two pipelined steps of $10 \mu \mathrm{s}$ each.

- Jets usually affect more than a single calorimetric tower. Calorimeter clustering (L2CAL) sums the energies collected by single towers and provides a measurement of the total jet energy.

- The calorimeter shower maximum (XCES) is used to reduce the rate of fake electrons and photons. It also makes it easier to match XFT tracks to their calorimetric clusters.

- The Silicon Vertex Tracker (SVT) reconstructs tracks in the vertex detector, measuring their impact parameter $d$. Triggering on $d$ proves extremely helpful in $b$-quark physics.

- Data is also collected from the level 1 track and muon triggers.

During the second pipelined step, the results of the first phase are fed to a set of Alpha processors; each processor examines the event for a different set of characteristics.

The level 2 accept rate is around $300 \mathrm{~Hz}$, with a rejection of about 150 .

\subsubsection{Level 3 trigger}

After being accepted by the level 2 trigger, the entire event data is read out and loaded into a Linux PC farm, where the event is fully reconstructed in software. The level 3 reconstruction program is almost fully written in $\mathrm{C}++$, using object-oriented techniques.

After an event is reconstructed, it is sent to an event counter, where its characteristics are histogrammed; if the event passes the level 3 cuts, it is also permanently stored to tape.

Assuming a level 3 input rate of $300 \mathrm{~Hz}$, a level 3 rejection of 10, and an average event size of $250 \mathrm{kB}$. 


\subsubsection{Online Monitoring}

The CDF detector consists of many detector subsystems and runs in a high rate large bandwidth data transfer environment. To take data with high efficiency and high quality, it is necessary to quickly spot problems with one of these sub-detectors in real time. Multiple event monitor programs are attached to the DAQ system [33][34][32]. The online monitoring programs are called Consumers, where a consumer is defined as a process which receives events from Consumer Server Logger (CSL) in real time. CSL sends the data to the computer center where they are written to tape and forwards copies of a subset of the data to the online monitoring programs. Figure 2.5 shows a schematic view of the CDF online monitoring system (Consumer Framework). The task of Consumers is to analyze and monitor the event data and to make histograms and tables. These results could be viewed by the display browser via a server in real time. Results of the monitor are also stored as data files periodically during a run, and also archived systematically. The display browser provides a GUI to view the online monitored results, while also providing some basic utilities to do comparisons with previously stored results. By separating the two tasks of monitoring and displaying, we remove CPU bound associated with displaying graphics from the machine which runs the consumers. During the data taking, multiple consumer processes run in parallel, receiving event data with the desired trigger types from the CSL. Communication between a consumer and run control which control overall CDF DAQ system is handled by the Error Receiver. Severe errors detected by a consumer monitor program are forwarded to run control to take necessary actions. The state manager watches the state of consumers. 


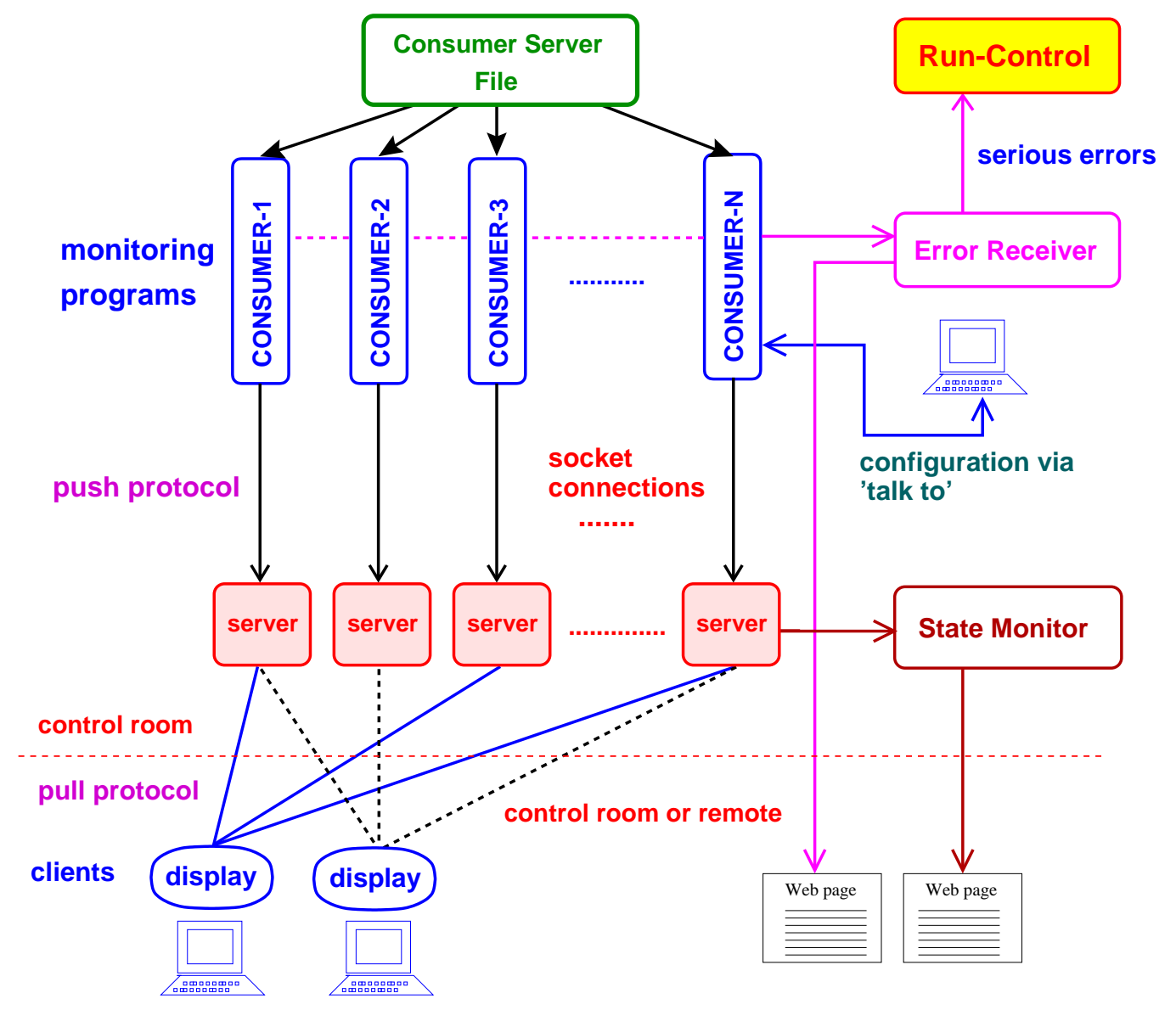

Figure 2.5: Design of the CDF online consumer framework. 


\section{Chapter 3}

\section{Particle Identification}

\subsection{Lepton Identification}

The identification of charged leptons produced from a $W$ decay is our first step in the selection of the $t \bar{t}$, lepton+jets sample. We require trigger conditions for the event to have either one electron or muon candidate. The trigger requirements will be described in more detail in Chapter 4.

\subsubsection{Identification of a Central Electron}

We require the central electron trigger for the event to be analyzed as one of our possible candidate events having an electron. In the decision of the central electron trigger, the a clustering of the energy deposit in the calorimeter is performed, and we require a electromagnetic (EM) cluster with $E_{T}>18 \mathrm{GeV}$ in the CEM region corresponding to $|\eta| \leq 1$

After passing the trigger requirement, an electron candidate is identified in the offline software with further selection cuts. We again require that the electron is found in the central pseudorapidity region of the detector $(|\eta| \leq 1)$ and have an EM cluster with $E_{T} \geq 20 \mathrm{GeV}$. This cluster energy in EM calorimeter is used as the energy of the electron candidate. A COT track with $p_{T} \geq 10 \mathrm{GeV} / c$ is required to match the EM cluster, in order to reduce mid-identification of photons and neutral pions. Several variables are used to further discriminate against charged hadrons and photon conversions. We describe some of the details below: We require that the extrapolated track reconstructed by COT hits matches the shower location as measured in the maximum detector (CES) [21]. To ensure a good quality of the track reconstruction, we require $\geq 3$ axial and $\geq 3$ stereo segments, $\geq 7$ segments in total to be hit by the track matching the electron cluster. The ratio of energy deposit in electromagnetic to hadronic calorimeters in the calorimeter cluster, $E_{h a d} / E_{e m}$ is less than 0.055 , and that the ratio of cluster energy to track 
momentum, $E / P$ is less than 2.0. The spacial distribution of the energy deposit is compared to and required to resemble that obtained in a beam test. The isolation, defined as the ratio of the additional energy deposits in a radius $\Delta R \equiv \sqrt{(\Delta \eta)^{2}+(\Delta \phi)^{2}}=0.4$ around the electron cluster to the electron energy, is required to be less than 0.1. In fact, we will perform the electron identification without requiring the isolation in the estimation of non- $W$ background. In other cases we always require the isolation.

Electrons originating from conversion of photons are removed by vetoing events that have a pair of tracks (one of them matching to the electron candidate) which satisfies (1) Opposite sign charges, (2) the distance between the tracks $\Delta(x y)$ in the $r-\phi$ plane where the tracks are parallel is less than $0.2 \mathrm{~cm}$, and (3) the difference between the polar angle cotangent of the two tracks, $|\cot \Delta \theta|$ is less than 0.04. Fiducial cuts on the electromagnetic shower position in CES are required to insure that the electron is away from the calorimeter boundaries to measure the energy well enough.

For isolated high momentum electrons from $\mathrm{W}$ decay, the tracking efficiency is measured to be $99.63{ }_{-0.40}^{+0.35} \%$ [35]. The transverse energy can be measured from the electromagnetic cluster with a precision $\sigma / E_{T}=13.5 \% / \sqrt{E_{T}(\mathrm{GeV})} \oplus 2 \%[25]$ and the transverse momentum resolution is found to be $\delta p_{T} / p_{T} \approx 0.15 \% \times p_{T}(\mathrm{GeV} / c)$.

\subsubsection{Identification of a Plug Electron}

We do not use an electron identified in the plug calorimeter in the reconstruction of the top quark mass, because such an electron candidate has a higher probability of a mis-identified hadron. However, we reject events that have two or more isolated high $P_{T}$ leptons identified as unlikely to be a lepton + jets event, and we consider the electron identified in the plug region in this di-lepton vetoing. We describe the identification of the electron in the plug calorimeter here $[36,37]$.

In order for an electron identification to be reliable, we require that a track is matched to the EM cluster. The tracking chamber (COT) is not efficient in the tracking in the high $|\eta|$ region because of the lack of geometrical acceptance. On the other hand, we must be able to reconstruct tracks up to the pseudo-rapidity of $|\eta|<1.8$ by using hits in the silicon detector in the track fitting, considerting the wide coverage in $|\eta|$ of the silicon detector. However, seeding tracks in the high $|\eta|$ region only with silicon hits is extremely difficult. In the tracking for plug electron identification, the seed track is constructed by interporating between the primary vertex and the shower-max hit in the EM cluster. Silicon standalone tracking is performed with the hits in the silicon detector based on the seed track thus reconstructed.

The starting point of the plug electron identification is an EM cluster with $E_{T}>20 \mathrm{GeV}$ in the PEM calorimeter. We require that a cluster in the shower-max deterctor (PES) with $1.2<|\eta|<2.5$, and that a plug electron track with $P_{T}>15 \mathrm{GeV} / c$ is reconstructed with more than 2 hits in the silicon detector. The lateral sharing of the energy deposit between the 
calorimeter towers is required to resemble that obtained in a beam test. The ratio of hadronic to electromagnetic energy deposite is required to be less than 0.05 . The isolation is required to be less than 0.1 as in the case of central electron identification.

\subsubsection{Identification of a Muon}

We require one of the muon triggers for the event to be analyzed as one of our possible candidate events having a muon. In the decision of the muon trigger, a track with $P_{T} \geq 18 \mathrm{GeV} / c$ that matches a muon stub in the muon chamber (CMU, CMP or CMX). This requirement corresponds to the pseudo-rapidity region of $|\eta| \geq 1.0$.

Muon candidates are identified by extrapolating the COT tracks to the muon detector. We have two types of high- $p_{T}$ muon sample used in this analysis. One is a track linked to a line(track) segment in both CMU and CMUP called a CMUP muon $(|\eta| \leq 0.6)$, and another type is a track linked to CMX called a CMX muon $(0.6 \leq|\eta| \leq 1.0)$. For both CMUP and CMX muons, we require the COT track with $P_{T} \geq 20 \mathrm{GeV} / / c$. The $P_{T}$ of the COT track is used as the measured momentum of the muon candidate. We further require that the energy in the calorimeter tower containing the muon is consistent with the deposit expected from a minimum ionizing particle to reject secondary particles in charged hadron showers in calorimeter which produce stubs in the muon chambers. Backgrounds from cosmic rays are removed by requiring that the distance of closest approach of the reconstructed track to the beam line, $d$ is less than $0.2 \mathrm{~cm}$.

For high momentum COT tracks, the resolution at the origin is $\delta z \approx 0.5 \mathrm{~cm}$ along the beam line and $\delta d \approx 350 \mu \mathrm{m}$ for the impact parameter in the transverse plane. And also the distance between the extrapolated track and the track segments in muon chamber, $\Delta x$ is required to be less than 3,5 and $6 \mathrm{~cm}$ for CMU, CMP and CMX respectively. Finally we make the isolation ratio cut with $I<0.1$. COT tracks are required to have at least 3 hits in both axial and stereo segments of COT, and is required to have at least 7 hits in total, as in the case of electron.

\subsection{Jet Identification and Corrections}

The jet reconstruction in this paper employs a cone cluster algorithm with cone radius $\Delta R \equiv$ $\sqrt{(\Delta \eta)^{2}+(\Delta \phi)^{2}}=0.4$ [38]. We measure the transverse energy $E_{T}=E \sin \theta$, where $\theta$ is the polar angle of the centroid of the clusters towers and calculated using the measured $z$ position of the event vertex. Total energy $E$ is the sum of the energy deposited in calorimeter towers within a cone. Jets are identified as isolated cluster and which contain significant hadronic energy where its measurements have a resolution of $\sigma / E_{T}=50 \% / \sqrt{E_{T}(\mathrm{GeV})} \oplus 3 \%[26]$.

Jet energies thus measured by the calorimeter cluster must be corrected for various detector effects before they are used in physics analyses. Our analysis apply two steps of corrections, one is flavor-independent generic corrections (simply called "generic correction"), the other is energy correction dependent on the flavor of the initial parton called "top specific correction". 
Flavor-independent generic corrections are found by a program of study which examines the transverse energy balance in the di-jet and the $\gamma+$ jet samples. At CDF, the jet energy correction has been studied in seven steps, each step corresponding to different effects. We simply list these steps of the corrections here [39] :

- Level 1 - The $\eta$ Dependence in Calorimeter Response: Correction to make the calorimeter response uniform in $\eta$ by using the di-jet balancing procedure and is tested by the $\gamma+$ jet samples in data and Monte Carlo simulation.

- Level 2 - Calorimeter Stability (Time dependence): Correction for the variation in phototube response due to aging effects, therefore the correction is applied only to the data sample.

- Level 3 - Raw Scale for Central Calorimeter Responses: Scaling from Run II to Run I energy scale by studying $\gamma$-jet balancing (jets in the central region). This is necessary because we still rely on the Run I parametrization for Level 5 correction (note that the central calorimeter of CDF was not replaced in the detector upgrade for Run II).

- Level 4 - Multiple Interaction: It is possible to have more than one interaction per collision due to high-luminosity collisions at Tevatron. This correction takes into account them. The correction is deduced from analyzing the minimum bias data as a function of the number of vertices in the same beam bunch crossing.

- Level 5 - Absolute Energy Scale: This correction accounts for the calorimeter non-linearity, fragmentation and underlying event (Using Run I correction factor).

- Level 6 - Underlying Event: The partons other than those involved in the hard collision (in our case it is the $t \bar{t}$ creation) in the $p \bar{p}$ system, called spectator partons, have reactions of their own and leave some energy in the calorimeter. This energy is called the underlying energy and must be subtracted. The underlying energy is measured from minimum bias data requiring events only one vertex.

- Level 7 - Out Of Cone Energy: It corrects the particle-level energy for leakage of radiation outside the clustering cone used for jet definition, taking the "jet energy" back to "parent parton energy". This correction allows us to correct the particle level jet energy to the parton level by taking into account energies from initial parton which are not included in jet clustering.

We used jet energies corrected up to level 4 in the event selection of our analysis. In the event reconstruction, we apply the generic correction up to level 5 . We further apply top specific corrections for this purpose, which will be described in chapter 5. Figure 3.1 shows typical generic correction scales for level 4 and level 5 corrections. 

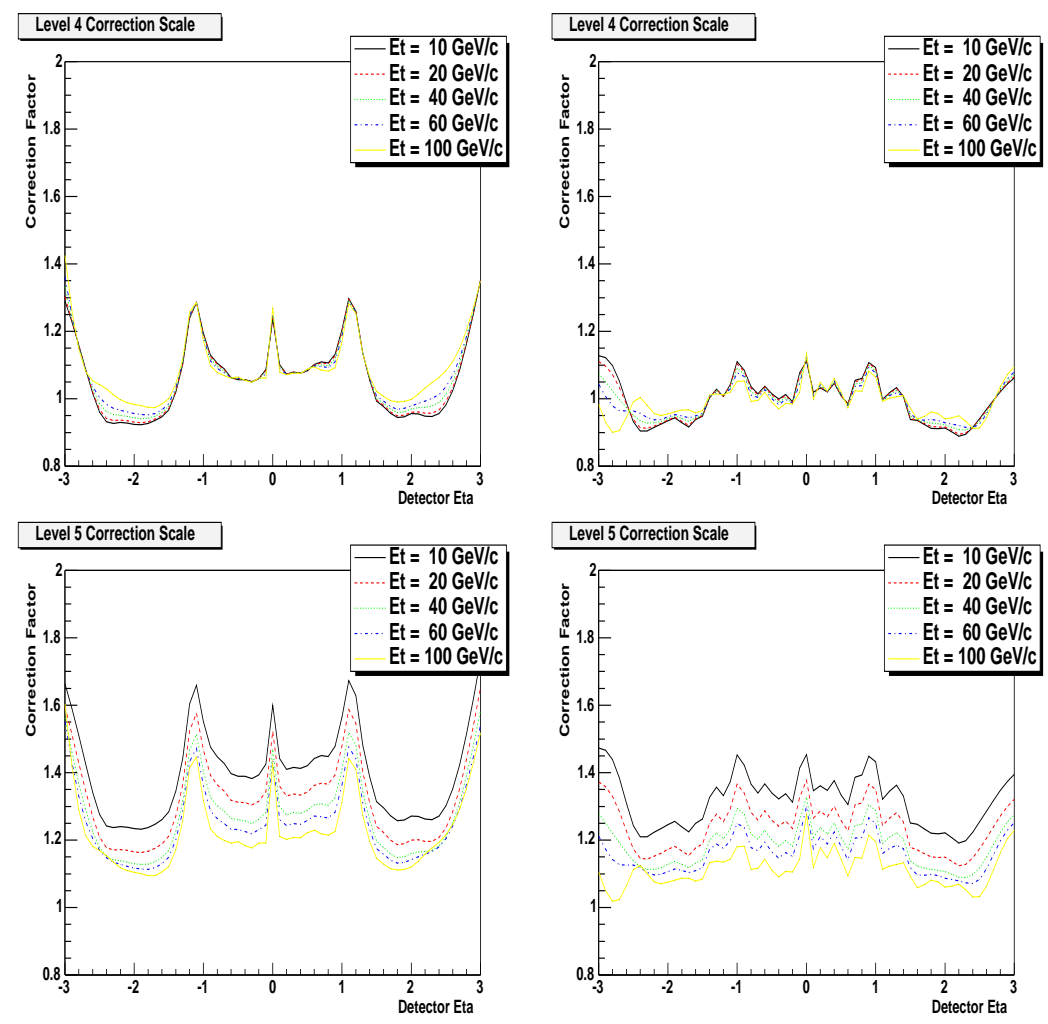

Figure 3.1: The correction scales for jet energy corrections. The left plots are the corrections applied to jets in collision data, and right plots are for Monte Carlo. The top plots are when we correct up to level 4, and the bottom plots are for level 5 . Correction scales for jets with several different $E_{T}$ are shown. These plots suppose the jets have EM energy fraction of 0.5 and the events have only one vertex.

\section{$3.3 \quad b$-Jet Tagging}

The identification of $b$ jets from top quark decay plays an important role in this analysis. Since most of the events coming from non- $t \bar{t}$ process passing all $\mathrm{W}+$ jets selection do not contain bottom or charm quarks in the final state, the requirement of presence of $b$ jets provides a significant reduction of the background events. We used two different $b$-tagging algorithms, SECVTX and Jet Probability, in order to achieve $b$-tagging conditions that is sensitive to the $t \bar{t}$ events.

\subsubsection{SECVTX algorithm}

The silicon vertex $b$ jet tag algorithm (SECVTX tagging) searches for the displacement of the secondary vertex due to $\mathrm{B}$ hadron decays to the primary vertex of the event within a jet $[40,41]$. It uses tracks which are within $\Delta R<0.4$ of the jet axis and have hits in the silicon detector. The algorithm performs as follows: (1) Select good tracks; which allows for two passes. In the first 
pass, at least three tracks with $p_{T}>0.5 \mathrm{GeV} / c$ and an impact parameter significance $\left|D_{0} / \sigma_{D_{0}}\right|>$ 2.5 are required where $\sigma_{D_{0}}$ includes the uncertainty on the track and beam-line positions. At least one of the tracks must have $p_{T}>1 \mathrm{GeV} / c$. In the second pass, which requires tighter track requirements to have at least two tracks with $p_{T}>1 \mathrm{GeV} / c$ and $\left|D_{0} / \sigma_{D_{0}}\right|>3$, including at least one track with $p_{T}>1.5 \mathrm{GeV} / c$. (2) Reconstruct a secondary vertex using selected tracks. (3) Calculate the two-dimensional decay length of the secondary vertex $\left(L_{x y}\right)$ from the primary vertex. (4) Require to have $L_{x y} / \sigma_{L_{x y}}>3$, where $\sigma_{L_{x y}}$ is the estimated uncertainty on $L_{x y}$ which is typically $190 \mu \mathrm{m}$ to reduce the background from the false secondary vertices(mistags).

Based on simulation of the $b$-tagging algorithm, the $b$-tag requirement keeps $53 \%$ of the top quark events while removing more than $95 \%$ of the background events. The difference between the efficiency in the simulation and that in the data has been measured using a $b$-enriched dijet sample in which a non-isolated electron is found in a jet. In fact, the efficiency in Monte Carlo simulation studies does not reproduce that in data analysis. We found data-to-Monte Carlo tagging efficiency scale factor of $0.82 \pm 0.06$ [41], which is used in Monte Carlo studies to better describe the phenomena in data. The error includes all systematic and statistical uncertainties.

\subsubsection{Jet Probability Tagging}

Jet Probability is an algorithm that is used to determine whether a jet is produced promptly at the primary interaction point, or from the decay of a long-lived particle [42]. This algorithm makes use of the information of the tracks that are associated to a jet to determine the probability for this ensemble of tracks to be consistent with coming from the primary vertex. The probability distribution of a primary jet is by construction uniformly distributed from 0 to 1 . For a longlived jet, the distribution peaks at 0 , which means that the probability of such jet to be primary is very small. Figure 3.2 illustrates the jet probability distributions from jets originating from light quarks, charm, and bottom jets. This probability is based on the impact parameters and their uncertainties of the tracks in the jet. The impact parameter of a track is assigned with a positive or negative sign depending on the direction of the track with respect to the jet axis. For the impact parameter in the $R-\phi$ plane, the sign is defined as

- $\operatorname{sign}=$ positive if $\cos \phi>0$.

- $\operatorname{sign}=$ negative if $\cos \phi<0$.

where $\phi$ is the angle between the jet axis and the track point of closest approach to the primary vertex (shown in Figure 3.3(left)). Another way of expressing this definition is

- $\operatorname{sign}=$ positive if the point of intersection of the track on the jet axis is in the same hemisphere as the jet direction.

- $\operatorname{sign}=$ negative if the point of intersection of the track on the jet axis is in the opposite hemisphere as the jet direction. 


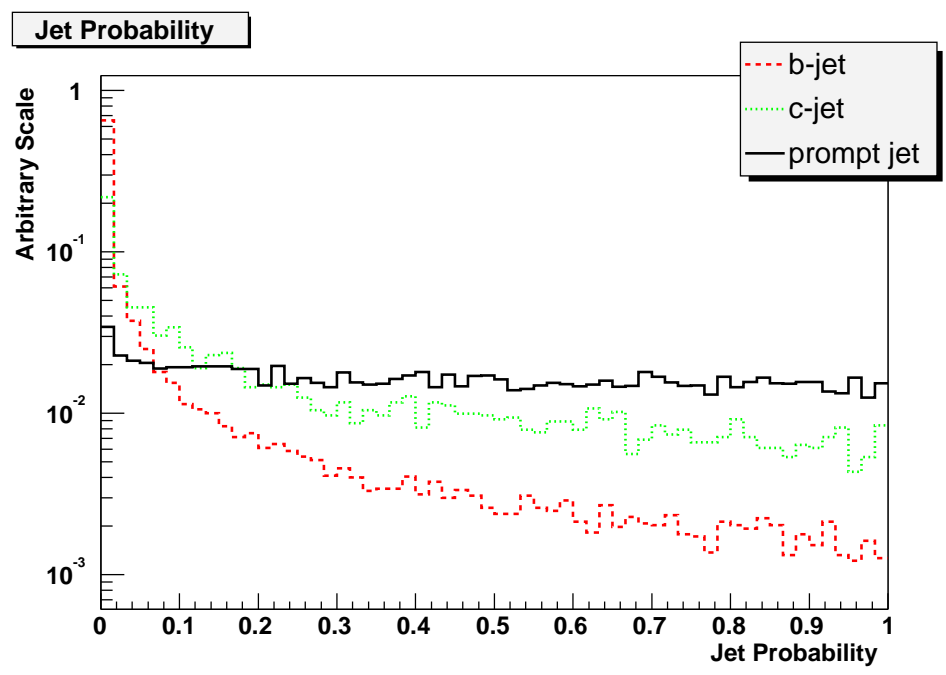

Figure 3.2: Jet Probability distributions from prompt, charm and bottom jets.

For a primary jet, all its particles should originate from the primary vertex. Due to the finite tracking resolution, these tracks are reconstructed with a non-zero impact parameter and have equal probability to be either positive or negative signed, as shown in Figure 3.4(left). The width of the impact parameter distribution from these tracks is solely due to the tracking detector resolution and multiple scatterings.

A long-lived particle will travel some distance along the jet direction before decaying, and its decay products will preferentially have positive signed impact parameter. An illustration of a long-lived particle and its decay tracks is shown in Figure 3.3 (right). The signed impact parameter distribution is shown in Figure 3.4(right).

The tracking resolution can be extracted from the data by fitting the negative side of the signed impact parameter distribution of primary jets. To minimize the contribution from badly measured tracks with large reconstructed impact parameters, the distribution of a related quantity, the signed impact parameter significance (ratio of the impact parameter to its measured error), is parameterized instead. Also, a quality cut on track is applied for the track to be used in the tagging of jets. This quality cut consists of the following conditions :

- $\operatorname{track} p_{T}>0.5 \mathrm{GeV}$.

- $\left|D_{0}\right|<0.15 \mathrm{~cm}$.

- more than 3 hits in $r-\phi$ layer of the SVX detector.

- total number of COT axial hits $\geq 20$.

- total number of COT axial hits $\geq 17$. 


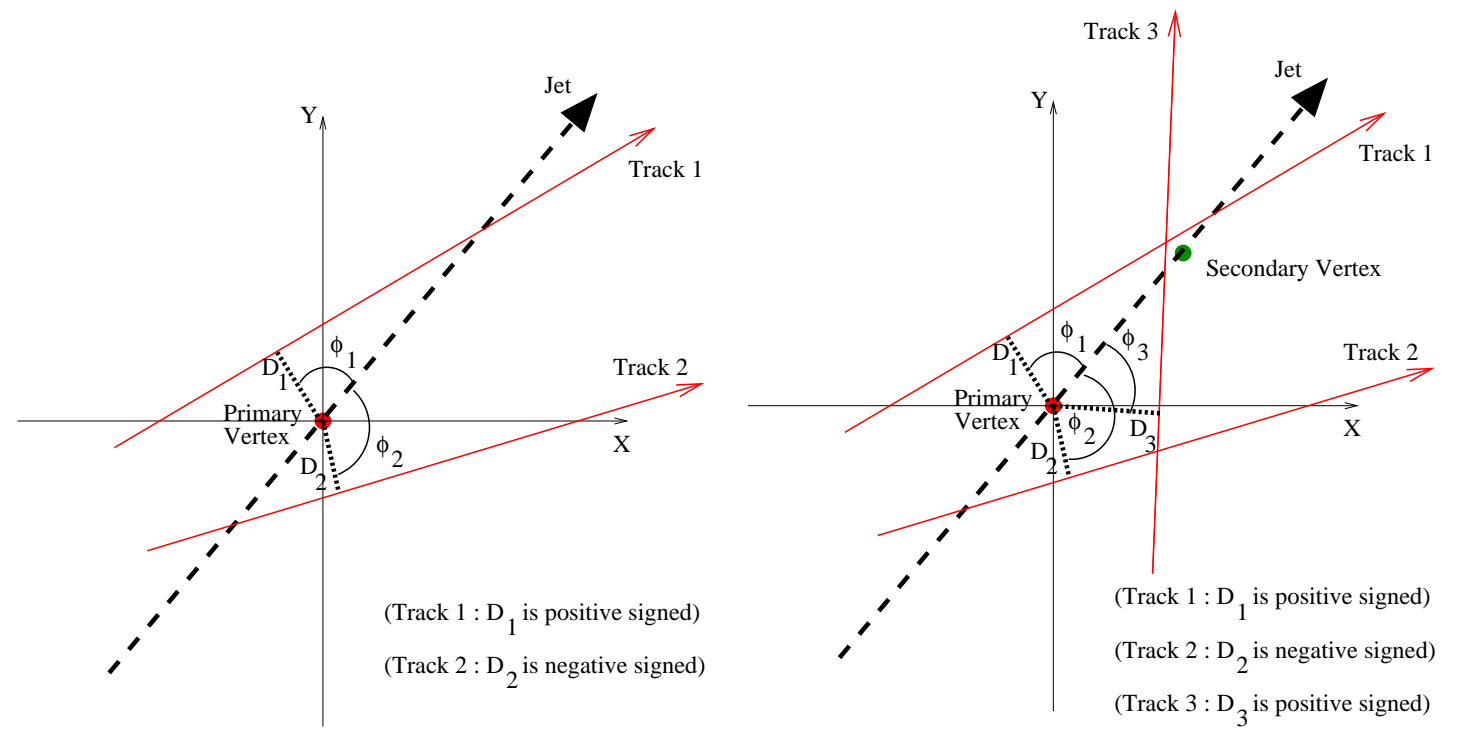

Figure 3.3: (Left) tracks from a primary vertex. (Right) tracks from a secondary vertex.

- $Z_{0}$ of track within $5 \mathrm{~cm}$ of the primary vertex.

We define a probability ("track probability") that a track in a jet originates from the primary vertex. Although tracks with negative signed impact parameters are used in the calibration of the algorithm, only tracks with positive signed impact parameters are used in the tagging of $b$-jets. For simplicity of the discussion, we do not try to explain the calibration here. For a track with positive impact parameter, the track probability is calculated as follows : First a signed impact parameter significance distribution for tracks in primary jets is extracted from a generic jet dataset. The positive part of the distribution is normalized so that the total area becomes 1 . For a given track, the track probability is defined as the area where the signed impact parameter is larger than that of this track, as illustrated in Figure 3.5. By definition, this variable has a flat distribution between 0 and 1 for tracks in primary jets. On the other hand, it has a peak around 0 for tracks in jets originating from heavy flavor partons.

Another new variable ("jet probability") is defined to characterize the probability of this jet originating from the primary vertex by combining the track probabilities of tracks in the jet. To see how we calculate the jet probability, consider a jet which has two good quality tracks with positive signed impact parameters in it. Suppose the two tracks each has track probability $P 1$ and $P 2$. Let $\Pi=P 1 \cdot P 2$. Figure 3.6 shows the curve of constant probability, and the area below and to the left of the curve is the set of two-track combinations with a probability less than or equal to $\Pi$. This area is defined to be the jet probability of this jet. For a two-track jet, the probability is $P_{j e t}=\Pi(1-\ln \Pi)$. In general, one can perform an integration in $n$-dimensional 

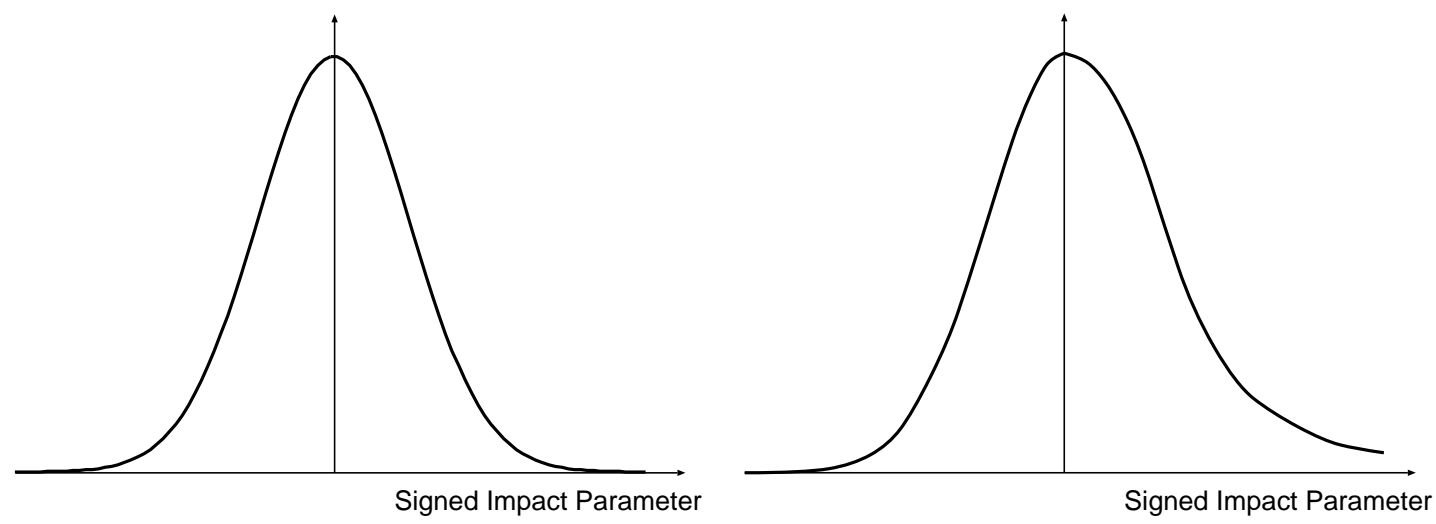

Figure 3.4: (Left) signed impact parameter distribution for tracks from primary vertex. (Right) signed impact parameter distribution for tracks from secondary vertex.

space and show that the jet probability for a jet with $n$ tracks would be [43]:

$$
P_{j e t}=\Pi \sum_{k=0}^{n-1} \frac{(-\ln \Pi)^{k}}{k !}
$$

where

$$
\Pi=P 1 \cdot P 2 \cdots P n
$$

Since the jet probability is defined in such a way combining the track probabilities, its distribution results similar to that of track probability. Indeed, as seen in Figure 3.2, the jet probability has a flat distribution for the light flavor (primary) jets, and has sharp peak around 0 for jets originating from heavy flavor partons (secondary jets). In the $b$-tagging with the Jet Probability algorithm, we can place an arbitrary cut, to select jets with jet probability values less than the cut value as likely to originate from heavy flavor partons. This tagger can, therefore, be tuned for each analysis topic rather easily.

This algorithm has been calibrated for two cut values of 0.01 and 0.05 in the CDF environment for jets with $E_{T}>10 \mathrm{GeV}$. Note that this $E_{T}$ is before the application of the jet energy correction. The results of the calibration averaged over the calibrated $E_{T}$ range is summarized in Table $3.1[44,45]$. For example, if we pick the cut of 0.05 , the tagger has the efficiencies of $26 \%$ and $4.9 \%$ for heavy and light flavor jets, respectively.

\subsection{Missing Transverse Energy; $\mathbb{F}_{T}$}

The presence of neutrinos is inferred from transverse energy imbalance in the detector. The missing transverse energy is calculated as :

$$
\#_{T}=-\left|\sum_{i} E_{T}^{i} \overrightarrow{n_{i}}\right|
$$




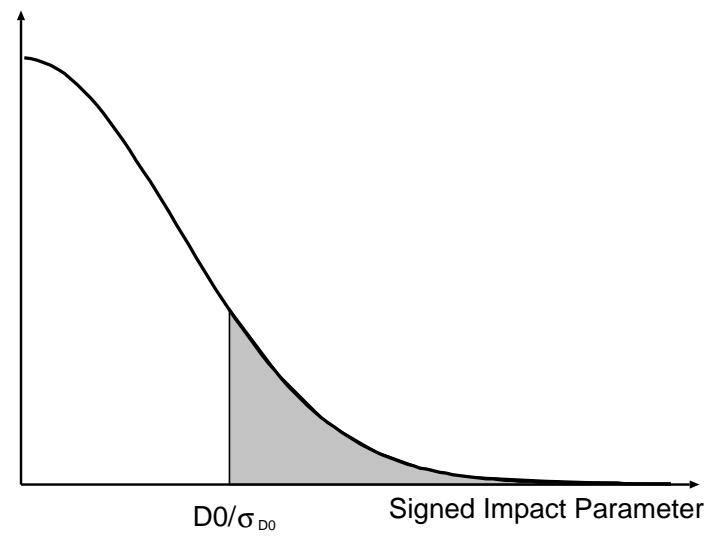

Figure 3.5: The positive part of the impact parameter significance of tracks in primary jets. Track probability is defined as the shaded area for given impact parameter significance when the total area is normalized to 1.

\begin{tabular}{|c|r|r|r|}
\hline \hline cut & efficiency for heavy flavor (\%) & scale factor & mistag rate (\%) \\
\hline 0.01 & $19.7 \pm 1.2$ & $0.787 \pm 0.105$ & $1.11 \pm 0.08$ \\
0.05 & $26.2 \pm 1.3$ & $0.820 \pm 0.095$ & $4.89 \pm 0.19$ \\
\hline \hline
\end{tabular}

Table 3.1: Summary of the Jet Probability tagger calibration. The scale factor is the ratio of efficiencies for heavy flavor jets between data and Monte Carlo. The efficiency for heavy flavor measured in data is shown here. The mistag rate corresponds to the efficiency of this tagger for light flavor jets.

where, $E_{T}^{i}$ is the magnitude of the transverse energy contained in each calorimeter tower $i$ and $\overrightarrow{n_{i}}$ is the direction of the tower in the plane transverse to the beam direction. If isolated high$p_{T}$ muons are found in the event, the $\mathbb{E}_{T}$ is corrected by subtracting the muon energy in the calorimeter and adding the muon $p_{T}$ back to the vector sum. When jets are corrected by generic correction, the $E_{T}$ must also be corrected so that the energy in the calorimeter balance. 


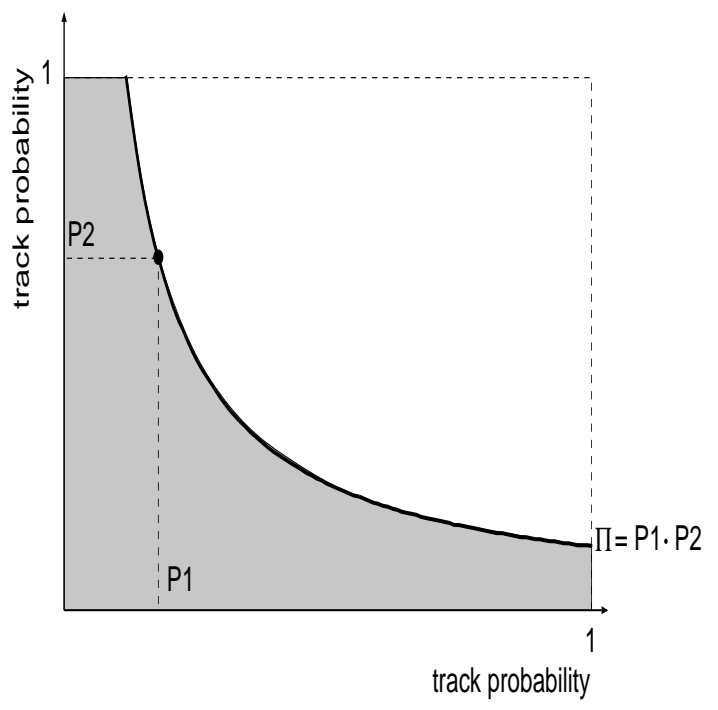

Figure 3.6: Definition of jet probability for a jet which has two good quality tracks with positive signed impact parameters in it. For a case where the tracks have track probabilities as shown in the dot, the jet probability is defined as the shaded area. 


\section{Chapter 4}

\section{Data Sample and Event Selection}

\subsection{Data Sample}

The results reported here are based on the data which were recorded by the CDF detector during the period March 2002 - August 2003, when the average instantaneous Tevatron luminosity was $\sim 2.0 \times 10^{31} \mathrm{~cm}^{-2} \mathrm{~s}^{-1}$. The highest recorded luminosity during this period was $4.2 \times 10^{31} \mathrm{~cm}^{-2} \mathrm{~s}^{-1}$. Our analysis further require that the data was taken with good operation condition of COT, calorimeter and muon detectors. The total integrated luminosity for this period is $193 \mathrm{pb}^{-1}$, of which $162 p b^{-1}$ has good silicon tracking along with good electron and CMUP muon reconstruction. Due to the large noise in the CMX muon chambers in the commissioning period of Run II, we did not use the events with CMX muon candidates in the corresponding period (until middle August 2002).x For CMX muon reconstruction, the corresponding integrated luminosity is $175 \mathrm{pb}^{-1}$, among which $150 \mathrm{pb}^{-1}$ had good silicon operation conditions.

Our analysis aims to reconstruct the top quark mass in the following physics process.

$$
p \bar{p} \rightarrow t \bar{t}+X \rightarrow W b W \bar{b}+X \rightarrow l \nu q \bar{q} ' b \bar{b}+X
$$

The most efficient way to reduce the huge amount of background events and make up an analysis data sample of suitable size is to identify the lepton (electron or muon) with high $P_{T}$. Therefore, we start with the events with either high $E_{T}$ electron or high $P_{T}$ muon identified in the trigger system of the CDF detector.

As described previously, the CDF employs a three level trigger system for high momentum electrons and muons analysis for the primary data sets. The first two triggers (Level 1 and Level 2) are hardware based online triggers and the third (Level 3) runs offline on a computer farm. For electron candidates, the level 1 trigger requires a track with $p_{T} \geq 8 \mathrm{GeV} / c$ matched to an EM calorimeter cell with $E_{T} \geq 8 \mathrm{GeV}$ and the ratio of the hadronic cell behind it to electromagnetic energy $\left(E_{h a d} / E_{\text {em }}\right)$ less than 0.125 . After calorimeter clustering is performed at level 2, it requires a track with $p_{T} \geq 8 \mathrm{GeV} / c$ matched to an EM cluster with $E_{T} \geq 16 \mathrm{GeV}$. At level 3 , electron cluster is reconstructed using calibration constants, and $E_{T}>18 \mathrm{GeV}$ is 
required. For muon candidates, a track with $p_{T} \geq 8 \mathrm{GeV} / c$ matched to muon stubs in the muon chamber CMU, CMP, and CMX is required through level 1 to level 2. And then level 3 trigger requires a $p_{T} \geq 18 \mathrm{GeV} / c$ track. We use the dataset passing through the trigger requirements described above as our data sample.

\subsection{Monte Carlo samples}

The simulation of $t \bar{t}$ events relies mainly on the HERWIG v6.5 [46] and PYTHIA v6.2 [47] Monte Carlo program which employ leading order QCD matrix elements for the hard process , followed by parton showering to simulate gluon radiation and fragmentation with the CTEQ5L [48] parton distribution functions. For heavy flavor jets, the decay algorithm QQv9.1 is used to provide proper modeling of $\mathrm{B}$ and $\mathrm{C}$ hadron decays. The ALPGEN program [49], which generates high multiplicity parton final states using exact leading-order matrix elements is used in our study of backgrounds in the b-tagged sample. The parton level events are then passed to HERWIG and QQ for fragmentation and B decay.

The CDF II detector simulation reproduces the response of the detector to particles produced in $p \bar{p}$ collisions. Tracking of particles through matter is performed with GEANT3 [50]. Charge deposition in the silicon detectors is calculated using a sample geometrical model based on the path length of the ionizing particle and an unrestricted Landau distribution. The drift model for the COT uses the GARFIELD package [23], with the default parameters tuned to match COT data. The calorimeter simulation uses the GFLASH [51] parameterization package interfaced with GEANT3. The GFLASH parameters are tuned to test beam data for electrons and high $p_{T}$ pions, and they are checked by comparing the calorimeter energy of isolated tracks in the collision data to their momenta as measured in the COT.

\subsection{Event Selection}

To reconstruct the top mass in each event, we reconstruct and identify all final particles of the top pair decay in Eqn. (4.1). In this section, we describe the selection criteria which we applied offline to identify the lepton+jets events.

Our event selection is decided to keep the consistency with other high $p_{T}$ lepton + jets analyses in CDF [52]. We require either one isolated electron or isolated muon captured in CEM, CMUP or CMX detector. So the coverage of the lepton identifications in this analysis is $:|\eta|<1.1$ for the electron, $|\eta|<1.0$ and for muon. Electron is required to have a EM cluster with $E_{T}>20 \mathrm{GeV}$, and muon is required to have a track with $P_{T}>20 \mathrm{GeV} / c^{2}$. Electrons originating from conversion and muons of cosmic rays are vetoed in the identification of the lepton. The details of the requirements are described in the previous chapter. The primary vertex of the event, which is defined by the closest approach of the track associated with the 
primary lepton to the beam line, must be within $60 \mathrm{~cm}$ from the center of the CDF detector. We reject events where another lepton is identified to have $P_{T}\left(E_{T}\right)>20 \mathrm{GeV}$ in order to reduce background coming from events where two gauge bosons are created in $p \bar{p}$ collision and both decay hadronically or $t \bar{t}$ events decaying into dilepton channel. We also reject events where isolated electrons with $E_{T}>20 \mathrm{GeV}$ are found in the plug calorimeter besides the primary lepton. We require the event to have missing $E_{T}\left(E_{T}>20 \mathrm{GeV}\right)$. Events are rejected as $Z$ boson candidates when a second object (EM cluster, opposite sign track with $E_{T}\left(P_{T}\right)>10 \mathrm{GeV}$ or jet with $E_{T}>15 \mathrm{GeV}$ and $|\eta|<2$ in the case of electron channel, opposite sign muon or track with $P_{T}>10 \mathrm{GeV}$ in muon channel) is found to form an invariant mass between 76 and $106 \mathrm{GeV} / c^{2}$ with the primary lepton.

The jets are clustered after removing towers containing electron clusters, and after correcting the tower $E_{T}$ for the location of the primary vertex $z$ coordinate. JETCLU algorithm with a cone size of 0.4 is used in the reconstruction, and we apply the generic jet energy correction up to level 4 for jet energies used in the event selection (for details of the generic jet correction, see chapter $3)$. For the $t \bar{t}$ event to be fully reconstructed, we require at least four jets to be reconstructed in the $|\eta|<2.0$ region of the calorimeter. We only consider these four selected jets in the $b$-tagging of the event as we describe later. For simplicity of the analysis, we suppose the four jets with the highest $E_{T}$ in the event to originate from the four quarks in the $t \bar{t}$ decay chain, and neglect the other jets found in the event. We divide our data sample into three subsamples - namely 0 , 1 and 2 tag samples - by the number of $b$-tags in the leading four jets. As for the leading three jets we require $E_{T}>15 \mathrm{GeV}$ while relaxing the cut for the fourth jet to have $E_{T}>8 \mathrm{GeV}$ in order to gain the efficiency for the single or double $b$-tagged event. On the other hand, the 0 tag channel has larger background fraction and we rather tightened the cut so that all the leading four jets have $E_{T}>21 \mathrm{GeV}$ and $|\eta|<2.0$ in order to have a good background reduction for this sample [54]. Although we do not further subdivide the data samples by the $E_{T}$ of the fourth jet in our analysis, we sometimes call events that has four jets with $E_{T}>15 \mathrm{GeV}$ "4-jet events", and events with $8<E_{T}^{4 \text { thjet }}<15 \mathrm{GeV}$ are called "3.5-jet events".

\subsection{Subdividing the Sample}

We subdivided our data and Monte Carlo samples selected by the kinematic cuts described in the previous section by the number of $b$-tagged jets into 0,1 , and 2 tag samples. In this section, we describe the tagging conditions for each category. The $E_{T}$ range of jets considered in the calibration of the $b$-tagging algorithms are $8 \mathrm{GeV}$ and $10 \mathrm{GeV}$ before the generic energy corrections for SECVTX and Jet Probability algorithms respectively. The typical correction factor for the level 4 correction was $1-1.2$ (chapter 3 ). Because we wanted to apply the $b$ tagging algorithms only in the calibrated $E_{T}$ region, we only applied the $b$-tagging to the jets with $E_{T}>15 \mathrm{GeV}$. We always consider jets with $8<E_{T}<15 \mathrm{GeV}$ as non-tagged. 
A goal of this analysis is to have the possible smallest uncertainty (stat + syst) on the measured top quark mass with combining the 0,1 and 2 tag samples. As we will discuss in section 5.3, $b$-tag information plays an important role in the correct reconstruction of the event. In fact the fraction of events we correctly reconstruct is the largest in the 2 tag sample. Moreover, the fraction of background events in the 2 tag sample is very small compared to 0 and 1 tag samples. Therefore, one of our main concerns is to gain high efficiency in the 2 tag channel by loosening the tag condition of this channel, and tune the double tag condition so that the uncertainty on the top quark mass becomes the smallest. For this purpose, we adopt the following $b$-tagging condition for the 2 tag sample.

- One jet is tagged by the SECVTX tagger.

- Another jet has either SECVTX tag or Jet Probability value $<J P$ cut, where $J P c u t$ is a certain threshold value.

This is to tightly tag one jet to secure enough purity for the 2 tag sample, and at the same time loosen the secondary tag to increase the efficiency for the double tagging. Note that SECVTX tagging has much smaller efficiency (mistag rate) for light flavor jets than Jet Probability algorithm at calibrated cut points, and also note that cut on Jet Probability is tunable. Events that has one SECVTX tagged jet and do not fulfill the 2 tag condition are categorized as 1 tag events. 0 tag sample consists of events where no jets are $b$-tagged by SECVTX tagger. In Monte Carlo studies, we randomly discard tags according to the scale factors given in [56] and [44] so as to match the tagging efficiency to that measured in data.

Other than the cuts we described in this chapter, we apply cuts on $\chi^{2}$ and $m_{j j}$ variables after the event reconstruction. When a 2 tag event fails either of these cuts, this event is merged into 1 tag sample in order to gain total efficiency of the event selection. This will be revisited in section 5.3. Figure 4.1 illustrates our sample subdivision.

We optimize this Jet Probability threshold (JPcut) to obtain the minimum top mass uncertainty in the data sample with 0,1 and $2 b$-tagged jets as described in section 8 .

In Figure 4.2, we display one of the candidate events found in our data sample that passed all the selection criteria of the $2 \mathrm{tag}$ sample. The sharp peak at $\eta \sim 0.1$ and $\phi \sim 250$ degrees on the top plot corresponds to the identified electron. One can see that there are other energetic activities in the event that are reconstructed into jets. The reconstructed tracks found in the jets are displayed around the interaction point in the bottom plot. This is an event with two SECVTX tagged jets, and one can see the decay length $L_{X Y}$ of the tagged jets in the plot. This event will be used in our mass measurement in the 2 tag sample. 


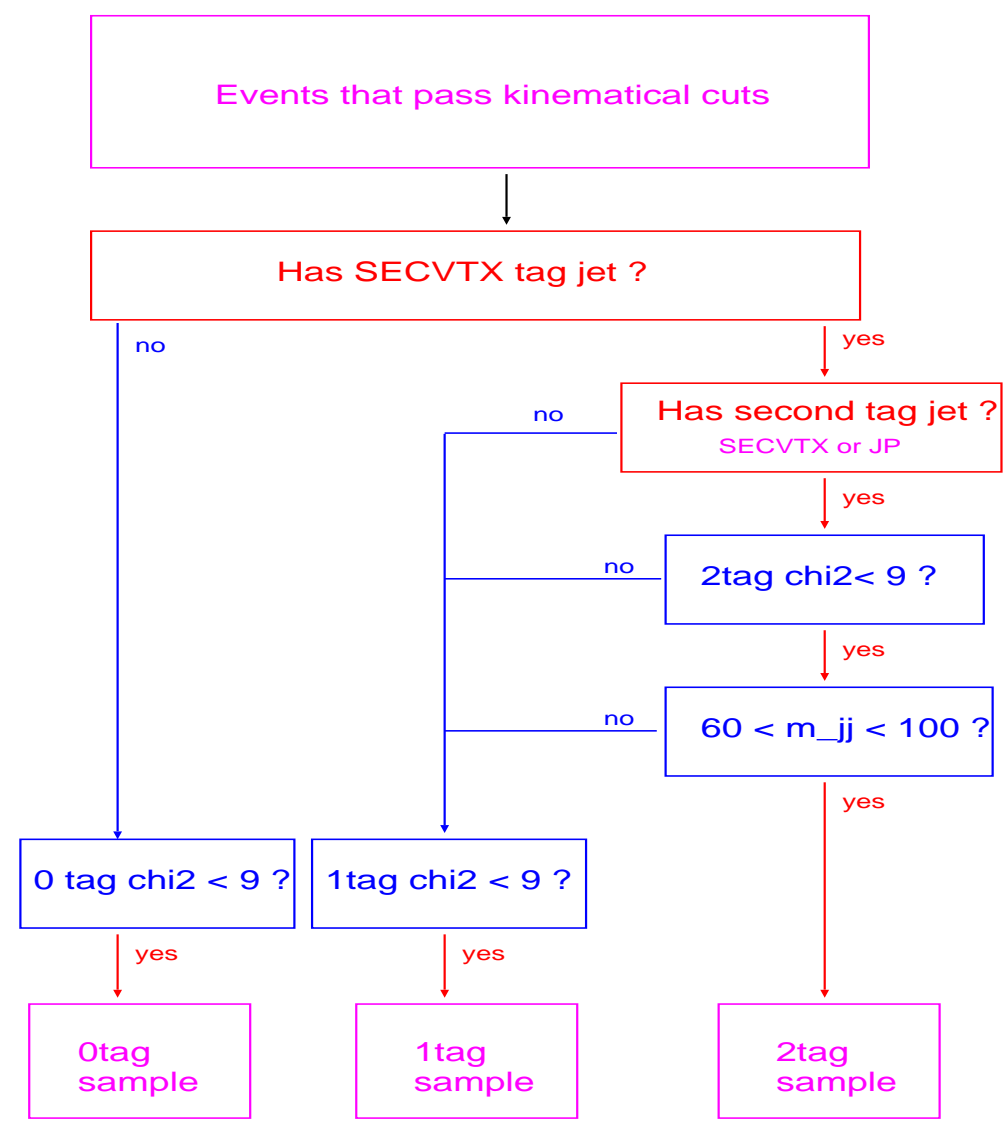

Figure 4.1: Subdividing the data sample. 

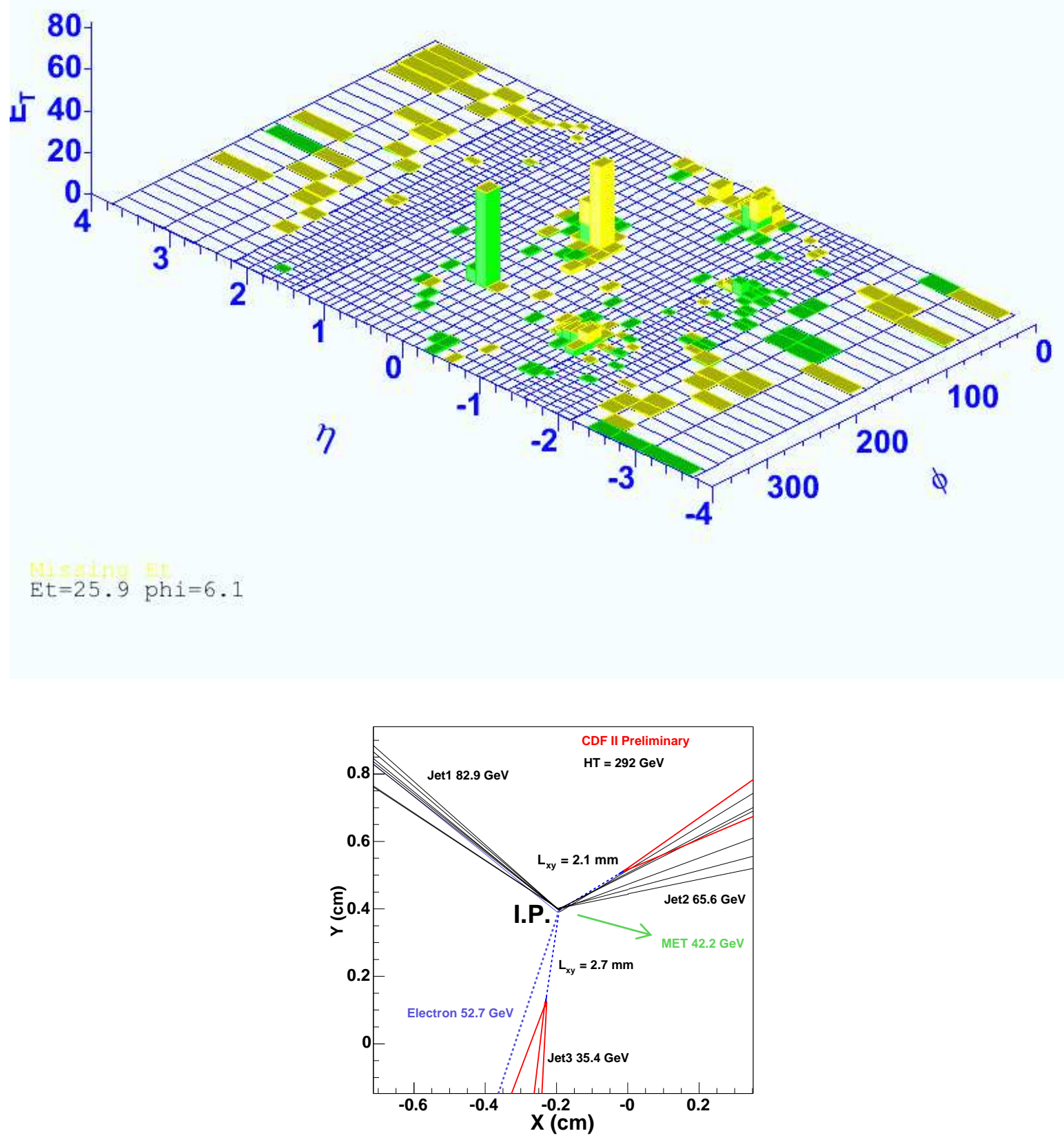

Figure 4.2: Event display for one of our candidate events found in data. Top : Energy deposit in each calorimeter tower is shown in the $\eta$ vs. $\phi$ plane. Bottom : The reconstructed tracks found in the jets. The reconstructed secondary vertices are also displayed. 


\section{Chapter 5}

\section{Top Quark Mass Reconstruction in each Event}

For each event that has been selected according to the previous chapter, we extract a single quantity through a series of corrections to the raw detector measurements and a multi-parameter fit utilizing MINUIT package [57]. This quantity is intended to have a strong dependence on the top quark pole mass, so that we can use its distribution in the data to determine the top mass through a likelihood fit to the distributions we extract from various Monte Carlo samples. Specifically, we do our best to reconstruct the invariant mass of the top quark in each event, assuming the event is $t \bar{t}$ signal with top quark decays and kinematics according to standard model predictions. Every event is treated identically, whether signal or background, data or Monte Carlo (except as noted below). Note that, although this quantity is referred to as the reconstructed top mass, it is not used as an independent measurement of the top mass in each event. It is simply an experimental quantity that will be used to discriminate among the range of possible true values of the standard model top quark mass.

\subsection{Correcting Measured Quantities}

We will provide as input to the MINUIT fit the four-vectors of the various physics objects identified in the event. We first apply all known corrections to the measured quantities, and establish an uncertainty on the quantities that will be permitted to vary in the fit.

\subsubsection{Lepton Corrections}

For both electrons and muons, we use the corrected four-vector calculated in the CDF software. In the case of electrons, this includes standard corrections for tower map response. The electron energy is the two-tower calorimeter energy, while the angles are taken from the beam-constrained COT-only track. The electron mass is set to zero in the four-vector, and the angles are taken as 
perfectly measured quantities. The transverse momentum $\left(p_{T}^{e}=p \sin \theta\right)$ of the electron is given an uncertainty of

$$
\sigma_{p_{T}^{e}}=\sqrt{\left(\frac{0.135}{p_{T}^{e}(\mathrm{GeV} / c)}+(0.02)^{2}\right)}
$$

Since the angles are fixed, this amounts to an uncertainty on the magnitude of the full electron momentum vector, not just the transverse component.

For muons, the corrected four-vector uses the three-vector of the beam-constrained COTonly track, also with a mass of zero. We apply in addition the muon curvature corrections due to chamber misalignment:

$$
\frac{1}{p_{T}^{\mu}(\mathrm{GeV} / c)}=\frac{1}{p_{T}^{\mu}(\mathrm{GeV} / c)}-0.00037-0.00110 \sin (\phi+0.28)
$$

(data only; no such correction is applied to Monte Carlo muons). Again the angle and mass are given no uncertainty; the transverse momentum is given an uncertainty of

$$
\frac{\sigma_{p_{T}^{\mu}}^{\mu}}{p_{T}^{\mu}}=0.0011 \cdot p_{T}^{\mu}(\mathrm{GeV} / c)
$$

which is equivalent to an uncertainty on $|\vec{p}|$, as for electrons. The electron and muon transverse momentum uncertainties are from run I.

\subsubsection{Generic Jet Corrections}

Jets are first corrected with the generic jet corrections up to level 5 , which is independent of the flavor of the jet. Generic jet corrections simply scale a jet four-vector by a factor, so angles are unaffected, while $\mathrm{E}$ and $\mathrm{p}$ change. The various sources of uncertainty in the CDF jet energy scale and simulation are quantified in the systematic uncertainties on these jet corrections, which will be considered in the systematic uncertainty estimation later. Details of the generic correction can be found in chapter 3 .

\subsubsection{Top-Specific Jet Corrections}

The generic jet corrections are extracted to uniformly correct the jet $E_{T}$ regardless of the flavor of the parton initiating the jet. It's extracted assuming a flat $E_{T}$ spectrum. Jet corrections derived specifically for the $t \bar{t}$ process (called top-specific correction) are needed to account for the different fragmentation of light quark jets from $W$-boson decay ( $W$ jets) and b jets, as well as the non-flat $p_{T}$ spectrum of jets from $t \bar{t}$ decay. In addition, the top-specific corrections account for the energy falling outside the jet cone. $W$ jets and $b$-jets are corrected with separate functions. The top-specific corrections also provide uncertainties on the jet energies (i.e. the measurement resolution, not systematic uncertainties). As for the lepton four-vectors, the angles of jets are assumed to be perfectly measured; only the $\sigma_{p_{T}^{j e t}}$ is parameterized. The top-specific 
corrections are functions of $p_{T}^{j e t}$ and $\eta^{\text {jet }}$. They are extracted from a large sample of HERWIG $t \bar{t}$ events in which the four leading jets in $E_{T}$ are matched with the four Monte Carlo partons from $t \bar{t}$ decay. As a crosscheck, the correction functions and their effect on the reconstructed invariant mass of the hadronic top quark have been shown to be consistent with those extracted from a large PYTHIA sample.
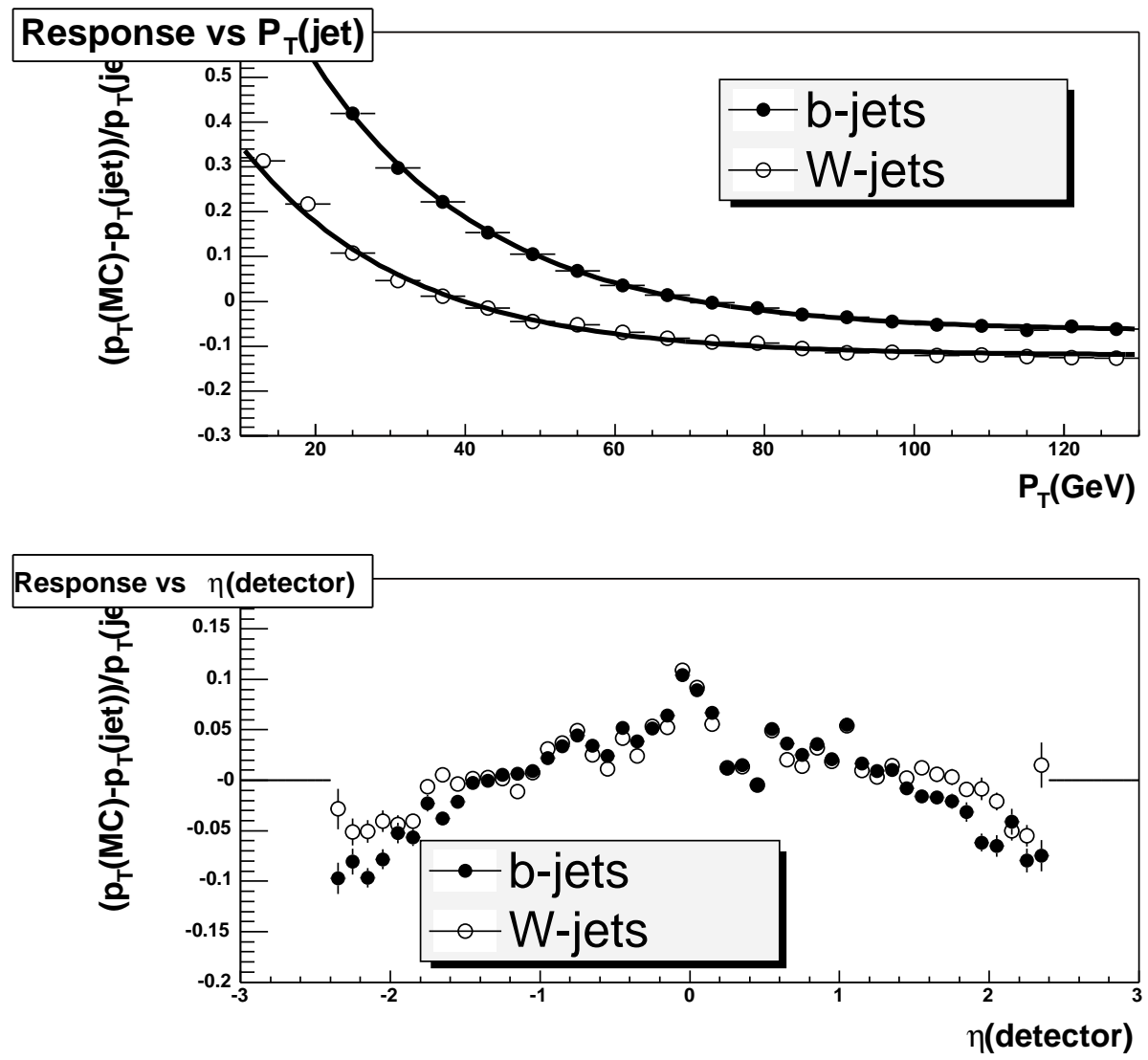

Figure 5.1: Upper plot: jet response, $\left(p_{T}^{\text {parton }}-p_{T}^{\text {jet }}\right) / p_{T}^{\text {jet }}$, as a function of $p_{T}^{\text {jet }}$ for $b$-jets and $W$-jets after only generic corrections are applied. Lower plot: jet response as a function of $\eta^{j e t}$ after applying top-specific corrections that depend only on $p_{T}^{j e t}$.

Figure 5.1 illustrates the need for top-specific corrections. The upper plot shows significant differences between parton momentum and jet momentum with $p_{T}^{j e t}<40 \mathrm{GeV} / c$ after generic corrections. The top-specific corrections remove the dependence of the jet response as a function of $p_{T}^{j e t}$ separately for $b$-jets and $W$-jets. In addition, the $p_{T}^{j e t}$ spectrum of jets from $t \bar{t}$ decay is strongly $\eta^{j e t}$-dependent. Therefore we expect $\eta^{j e t}$ dependence in the jet response after any top-specific corrections that depend only on $p_{T}^{j e t}$. This is illustrated in the lower plot of Figure 5.1. We hired a top-specific correction extracted as a function of both $p_{T}^{j e t}$ and $\eta^{j e t}$ (called JF correction) so as to remove this effect in our analysis. 


\subsubsection{Additional Jets, Unclustered Energy, and Missing Energy}

"Additional jets" in an event are those jets beyond the generic corrected $E_{T}$, but still having $E_{T}>8 \mathrm{GeV}$ and $|\eta|<2.0$. They are identified as not belonging to the top decay chain, but arising from initial state radiation, underlying event, or multiple interactions. This is, of course, an approximation, since the additional jets may in fact be top decay daughters, or may arise from final state radiation and therefore be relevant in reconstructing the top quark mass. The "unclustered energy" in an event consists of any energy seen in the calorimeter that is not associated with the primary lepton or a jet. The $\mathrm{p}$ of towers with $|\eta|<3.6$ are included in the unclustered energy. The "missing energy" is identified with the energy carried out of the detector by one or more neutrinos. The unclustered energy and missing energy are related to each other through the other measured physics objects in the event, since we assume that the $p \bar{p}$ system has $\overrightarrow{p_{T}}=0$.

In this analysis, the input to the MINUIT fit called unclustered energy is the sum of the raw unclustered energy, described above, and the energies of the additional jets. We add to the unclustered energy the energy that would have been subtracted from the leading four jets due to the underlying energy, and we subtract the energy that would have been added to those jets due to energy falling outside the jet cone. This is done to avoid double-counting of energy that is included in the jet energies after all corrections. The raw unclustered energy is then multiplied by a scale factor of 1.4, which is roughly the generic correction factor for $8 \mathrm{GeV}$ jets. So the unclustered energy input to the fit is

$$
\vec{U}_{T}=1.4 \times{\overrightarrow{U_{T}}}^{\text {raw }}+\Sigma_{j e t=1}^{4}{\overrightarrow{E_{T}}}^{\text {underlying }}-\Sigma_{j e t=1}^{4}{\overrightarrow{E_{T}}}^{\text {outofcone }}+\Sigma_{\text {additionaljets }}{\overrightarrow{E_{T}}}^{\text {jet }}
$$

Each transverse component of this composite unclustered energy $\left(U_{x}, U_{y}\right)$ is assigned an uncertainty of $0.4 \cdot \sqrt{E_{T}^{u n c l u s}}$.

The neutrino energy, i.e. the missing energy, is always calculated from the other objects, including the composite unclustered energy. In practice, only the transverse momentum in

$$
\overrightarrow{p_{T}}=-\left(\overrightarrow{p_{T}}+\Sigma p_{T}^{\overrightarrow{j e t}}+\overrightarrow{U_{T}}\right)
$$

The longitudinal momentum of the neutrino $p_{z}^{\nu}$ is a free (unconstrained) parameter in the fit. Its initial value is calculated using the initial value of the lepton four-vector and the initial missing energy, and assuming their mother $W$ has its pole mass. Since these conditions yield a quadratic equation, there are in general two solutions for the $p_{z}^{\nu}$. When the two fits land in different minima, we choose the one that provides smaller $\chi^{2}$.

\section{$5.2 \quad \chi^{2}$ Fitter}

Given the inputs described above, the event-by-event fit for the reconstructed top mass proceeds as follows. MINUIT is used to minimize a $\chi^{2}$ where the top mass is a free parameter. For each 
event this is done 24 times, corresponding to the 12 ways to assign the four leading jets to the four partons in $t \bar{t}$ decay as we will discuss in the next section, times the 2 solutions for the neutrino longitudinal momentum. For each combination, the following $\chi^{2}$ is minimized:

$$
\begin{aligned}
\chi^{2}= & \Sigma_{i=l, 4 j e t s} \frac{\left(p_{T}^{i, f i t}-p_{T}^{i, \text { measured }}\right)^{2}}{\sigma_{i}^{2}}+\Sigma_{j=x, y} \frac{\left(U_{T}^{j, f i t}-U_{T}^{j, \text { measured }}\right)^{2}}{\sigma_{j}^{2}} \\
& \frac{\left(M_{j j}-M_{W}\right)^{2}}{\Gamma_{W}^{2}}+\frac{\left(M_{l \nu}-M_{W}\right)^{2}}{\Gamma_{W}^{2}}+\frac{\left(M_{j j b}-M_{t}\right)^{2}}{\Gamma_{t}^{2}}+\frac{\left(M_{l \nu b}-M_{t}\right)^{2}}{\Gamma_{t}^{2}}
\end{aligned}
$$

In the first term, $\sigma_{l}$ and $\sigma_{j e t}$ are the lepton and four leading jet $p_{T}$ resolutions. In the second term, $U_{T}^{x, y}$ and $\sigma_{x, y}$ are the components of the composite unclustered energy and their error. Each of $M_{l \nu}, M_{j j}, M_{j j b}$ and $M_{l \nu b}$ refers to the invariant mass of the sum of the four-vectors denoted in the subscript. For example, $M_{j j}$ is the invariant mass of the vector sum of the W daughter jets. $M_{W}$ and $M_{t}$ are the pole masses of the $W$ boson and top quark. $M_{W}$ is $80.41 \mathrm{GeV} / c^{2}$ taken from the world average of direct measurements, and $M_{t}$ is, of course, the

free parameter that we will take as the reconstructed mass after the $\chi^{2}$ is minimized. The fit is initialized with $M_{t}=175 \mathrm{GeV} / c^{2} . \Gamma_{W}$ and $\Gamma_{t}$ are the total width of the W boson and the top quark. $\Gamma_{W}$ is $2.06 \mathrm{GeV}$ and $\Gamma_{t}$ is $2.5 \mathrm{GeV}$. Thus these terms provide constraints such that the $W$ masses come out correctly, and the $t$ and $\bar{t}$ masses come out the same.

\subsection{B-Tagging Conditions and the Choice of the Fit Results}

There are twelve possible combinations in assigning each jet to each parton in the top pair decay (four choices of assigning a jet to the $b$ quark in semileptonic top decay, three possible assignment of the jet to the $b$ quark in hadronic top decay, and we cannot distinguish between the two $W$ decay partons). Supposing one of these combinations, we still have two $p_{z}$ solutions for the neutrino. Thus there are in total twenty-four possible reconstructions for each event. MINUIT routine outputs the minimized $\chi^{2}$, the momenta of the six final particles and the top quark mass corresponding to the minimized $\chi^{2}$ for each reconstruction.

In choosing one solution out of the twenty-four reconstructions, we first discard the reconstruction that contradicts with the $b$-tagging information. If $b$-tagged jets are assigned to partons from $W$ boson decay we consider this is contradictory. This reduces the number of possible reconstructions down to four (two for the jet-parton combination and two for $p_{z}^{\nu}$ choice) for the double $b$-tagged events, while to twelve for the one tag. In the case where we have three or more $b$-tagged jets among the leading four jets, it is possible that this is a $t \bar{t} \rightarrow l+j$ ets event with one of the $W$ bosons decaying into charm and strange quarks, with the charm jet tagged besides the two $b$-jets. In such a case, we force this event to be a 2 tag event due to the following.

- If the event has more than two SECVTX tagged jets, we pick the two SECVTX tagged jets highest in $E_{T}$ as $b$-jets and discard other tagging information. 
- If the event has one SECVTX tagged jet, we pick the $b$-tagged jet with the smallest Jet Probability value as the second $b$-tagged jet, and discard other jets tagged by Jet Probability.

These conditions were defined based on the fact that SECVTX has much less mistag rate. We choose the reconstruction satisfying this consistency cut that gives the smallest $\chi^{2}$ as the "seemingly correct" reconstruction. We discard events with $\chi^{2}>9$ as likely to be background events.

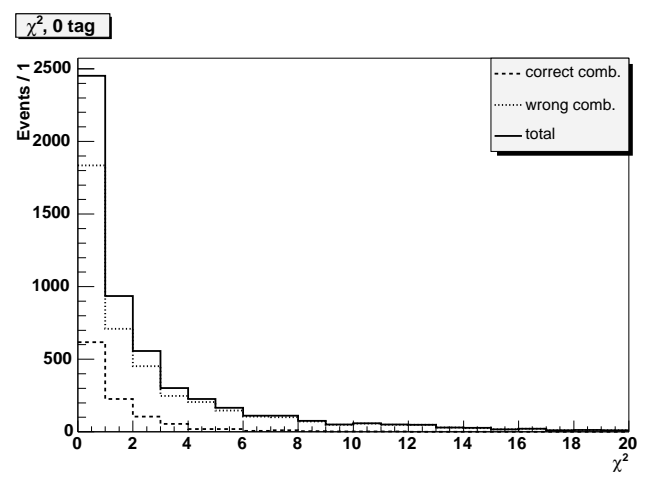

Figure 5.2: Distribution of $\chi^{2}$ in 0 tag sample, before the $\chi^{2}$ cut for $t \bar{t}$ Monte Carlo events. Distributions for correct and wrong combinations are also shown.

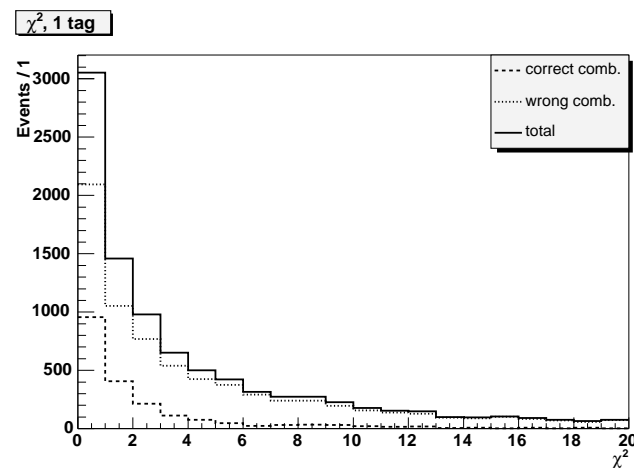

Figure 5.3: Distribution of $\chi^{2}$ in 1 tag sample, before the $\chi^{2}$ cut for $t \bar{t}$ Monte Carlo events. Distributions for correct and wrong combinations are also shown.

Figures 5.2, 5.3 and 5.4 show the distributions of the $\chi^{2}$ for 0,1 and 2 tag samples in $t \bar{t}$ Monte Carlo events. Distributions for events where the correct or wrong parton-jet assignment was chosen are also shown. For a comprehensive description of the categorization of correct and wrong combinations, see Section 5.4. We can see that the correct combination has almost no entry at the $\chi^{2}$ cut value of 9 , while this cut rejects some events with wrong reconstruction. 


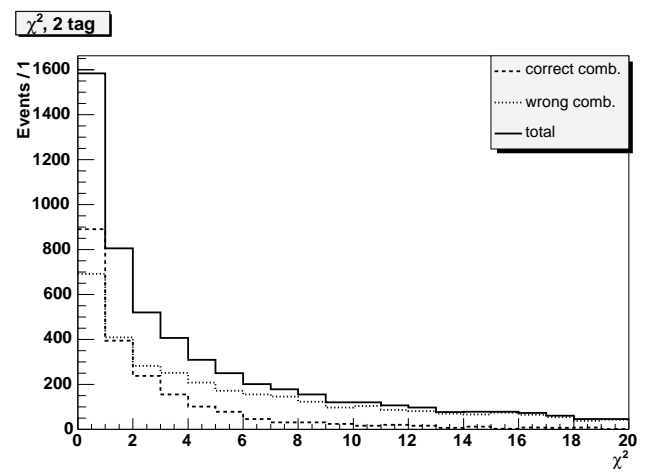

Figure 5.4: Distribution of $\chi^{2}$ in 2 tag sample, before the $\chi^{2}$ cut for $t \bar{t}$ Monte Carlo events. Distributions for correct and wrong combinations are also shown.

One of the most important impacts of requiring two $b$-tagged jets is that we can reliably reconstruct the $W$ boson that decays hadronically. Although a constraint on the hadronic $W$ mass is included in the $\chi^{2}$ definition and it is as sharp as $\Gamma_{W}$ when one looks at the output of the MINUIT fitter, we further constrain it at the final stage of our event selection for 2 tag sample by requiring $60 \mathrm{GeV} / \mathrm{c}^{2}<m_{j j}<100 \mathrm{GeV} / \mathrm{c}^{2}$, where $m_{j j}$ is the invariant mass of the two untagged jets using the variables after the application of generic and top-specific corrections (We apply generic jet correction up to level 5 in the event reconstruction, followed by the corresponding top-specific correction).

In the dataset used in this analysis, the number of candidate events in each sample is expected to be small. Furthermore, Jet Probability tagging in the 2 tag condition sometimes makes the reconstruction with minimum $\chi^{2}$ different between 1 and 2 tag event reconstruction. Therefore, 2 tag events failing one of $\chi^{2}$ or $m_{j j}$ cuts is fed back to the 1 tag sample - we refer to this as "recycling of 2 tag event" - and undergo the $\chi^{2}$ cut again with only SECVTX tagging condition considered. By enabling such recycling, the sum of the numbers of events in 1 and 2 tag samples always stay the same, regardless of the cut on Jet Probability. For the 0 and 1 tagged events, we only apply the $\chi^{2}<9$ cut other than the cuts described in chapter 4 , and do not apply the $m_{j j}$ cut.

\subsection{Characteristics of the Sub-samples}

In this section, we present results from our Monte Carlo studies concerning our event selection cuts and our event reconstruction. All the results presented in this section are for JPcut $=0.05$. 


\subsubsection{Efficiency of Sample Selection}

Summarized in Table 5.1 is the efficiency of each cut, with the number of $t \bar{t}$ events generated by the Monte Carlo generator as the denominator. The efficiency includes the efficiencies of all the precedent cuts. We used the HERWIG Monte Carlo events generated assuming top mass to be $175 \mathrm{GeV} / c^{2}$, and the errors are for the Monte Carlo statistics. In this table, the denominator is always the total number of generated Monte Carlo $t \bar{t}$ events (the efficiencies here include the branching ratio of $t \bar{t} \rightarrow l+j e t s)$. Efficiencies for 2 tag channel but without the use of Jet Probability is also shown for reference (2 SECVTX). At the "kinematic" cut stage, three jets with $E_{T}>15 \mathrm{GeV}$ and fourth jet with $E_{T}>8 \mathrm{GeV}$ is required in relation to the jet energies. The $E_{T}^{4 \text { th jet }}>21 \mathrm{GeV} / c^{2}$ cut for $0 \mathrm{tag}$ sample is applied at the "tag" stage. Numbers in tag, $\chi^{2}$ columns for $1 \mathrm{tag}$ sample include the events recycled from the 2 tag sample. To be noted is that we gained $83 \%$ additional efficiency for 2 tag sample by including Jet Probability tagging where the threshold JPcut is 0.05 . The efficiency of our cut is $\sim 1 \%$ for 2 tag sample. Assuming $t \bar{t}=6.7{ }_{-0.9}^{+0.7} \mathrm{pb}[58]$, we expect to have $\sim 11 t \bar{t}$ signal events at an integrated luminosity of $162 \mathrm{pb}^{-1}$ in this channel.

\begin{tabular}{|c||c|c|c|c|}
\hline \hline cut & $0 \operatorname{tag}(\%)$ & $1 \operatorname{tag}(\%)$ & $2 \operatorname{tag}(\%)$ & 2 SECVTX (\%) \\
\hline \hline kinematic & \multicolumn{4}{|c|}{$7.19 \pm 0.04$} \\
\hline $\operatorname{tag}$ & $1.41 \pm 0.02$ & $2.75 \pm 0.03$ & $1.77 \pm 0.02$ & $0.79 \pm 0.01$ \\
\hline$\chi^{2}$ & $1.29 \pm 0.02$ & $2.09 \pm 0.02$ & $1.14 \pm 0.02$ & $0.53 \pm 0.01$ \\
\hline$m_{j j}$ & - & - & $0.97 \pm 0.02$ & - \\
\hline \hline
\end{tabular}

Table 5.1: The efficiencies of our event selection. See text for detailed description.

\subsubsection{Study of Combinatorial Background}

Another interesting study would be to understand the fraction of $t \bar{t}$ events correctly reconstructed, and to compare the behavior of the correctly reconstructed events to events with wrong reconstruction. In order to determine whether an event is correctly reconstructed, we match each jet used in the event reconstruction to a quark produced in the decay of the $t \bar{t}$ pair by requiring $\Delta R$ (parton, jet $)<0.4$. When none of the four partons match the jet, this jet is considered to come from a gluon produced through initial or final QCD radiation. We define the "correct" event reconstruction as the case in which the parton-jet assignment in the event reconstruction is consistent with the information obtained in the parton-jet matching. All the other events are defined as with "wrong" reconstruction. These events are also called "wrong combination" events, or "combinatorial background". By definition, all the events with gluon jets coming in the leading four jets are categorized as wrong combination. 


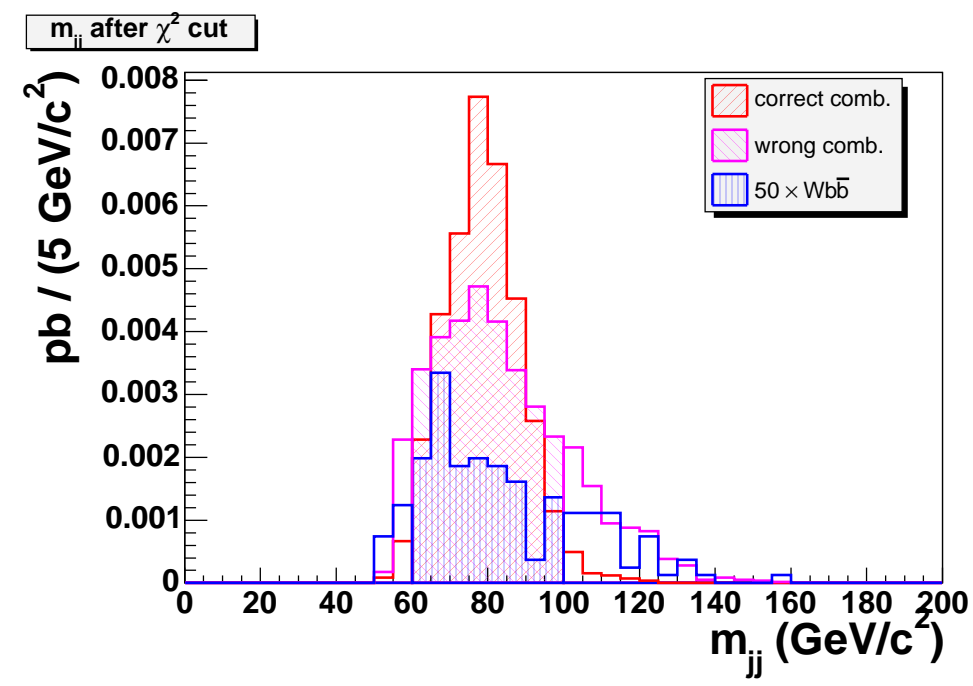

Figure 5.5: The invariant mass distributions of two jets assigned to light flavor partons by the top mass reconstruction module in Monte Carlo $t \bar{t}$ and $W b \bar{b}$ events. Histogram for $W b \bar{b}$ is scaled by a factor of 50 to be viewable. Hatched areas show the events satisfying the $m_{j j}$ cut.

Illustrated in Figure 5.5 is the distribution of the $m_{j j}$ variable for correctly and wrongly reconstructed $t \bar{t}$ Monte Carlo events (HERWIG, $m_{t}=175 \mathrm{GeV} / c^{2}$ ) in 2 tag sample along with the $W b \bar{b}+2$ parton Monte Carlo events generated by ALPGEN. $W b \bar{b}$ is one of the dominant background processes for the 2 tag sample. We show the events satisfying the $m_{j j}$ cut by hatched areas. The efficiencies of this cut for each category of events is summarized in Table 5.2. Although the $\chi^{2}$ definition includes a constraint on the mass of the $W$ boson decaying hadronically in the event, this cut works to tighten the event selection and is still very effective for further reducing the combinatorial background. Note that we use the "fitted" four momentum of the light flavor jets in the $\chi^{2}$ definition, while this cut uses the "measured" jet four momentum.

\begin{tabular}{|c|r|}
\hline \hline process & efficiency (\%) \\
\hline correct comb. & $95.5 \pm 0.5$ \\
wrong comb. & $75.6 \pm 1.0$ \\
$W b \bar{b}$ & $67.0 \pm 4.3$ \\
\hline \hline
\end{tabular}

Table 5.2: Efficiency of $m_{j j}$ cut for each category of Monte Carlo events.

In Figure 5.6, we show the reconstructed top mass distributions for different tagging requirements, along with the distributions only with the events correctly reconstructed. We can see that we drastically reduce the combinatorial background by tightening the tagging requirement in the event selection. $50.4 \pm 0.8 \%$ of our 2 tag sample after the $m_{j j}$ cut are the correctly 
reconstructed events. Note that the "wrongly" reconstructed events $(\sim 50 \%)$ include events
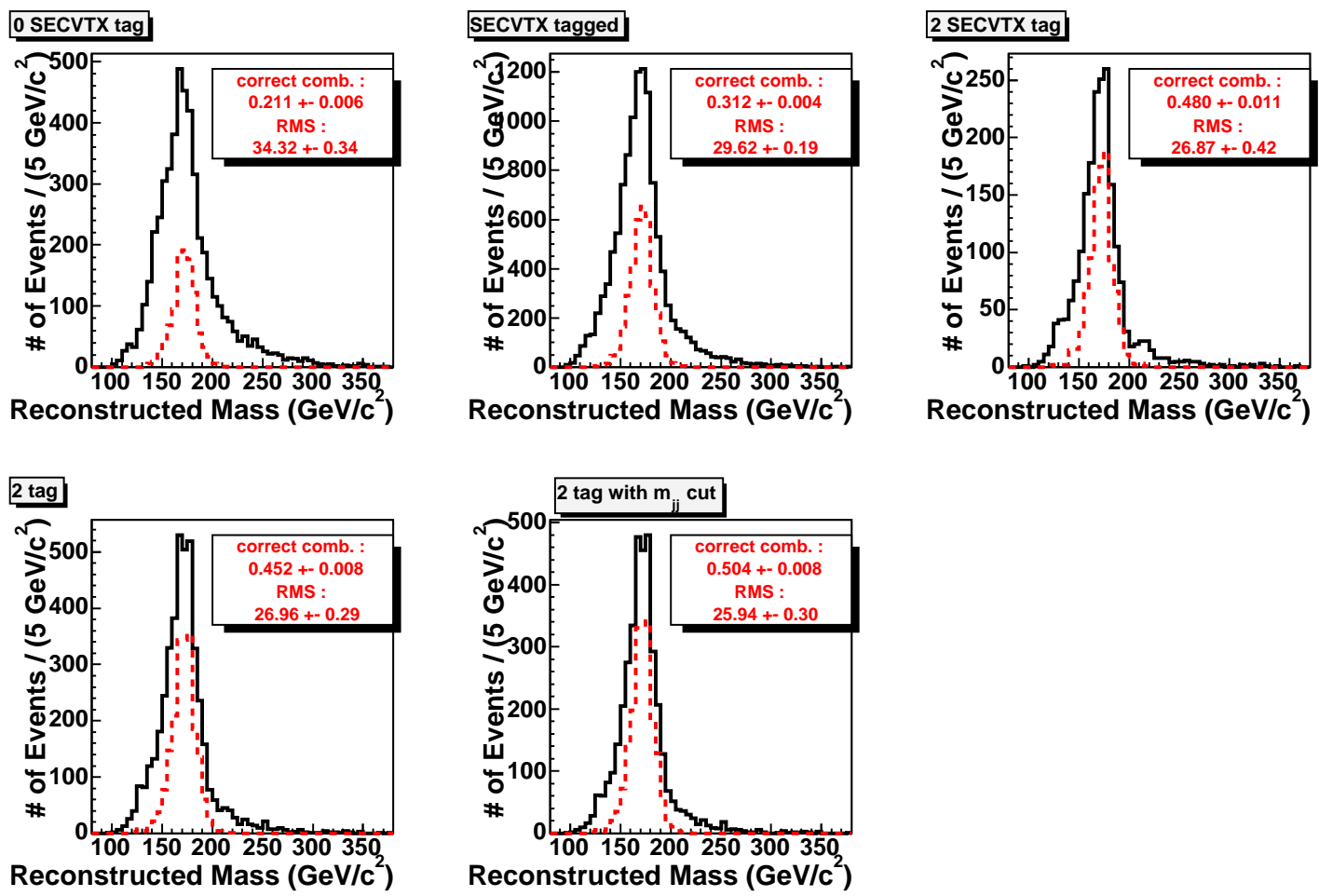

Figure 5.6: The reconstructed top mass distributions after applying all cuts in the $m_{t}=$ $175 \mathrm{GeV} / c^{2}$ Monte Carlo sample. For each plot, the solid histogram is for all the events, and the dashed histogram is for events where the $t \bar{t}$ decay was correctly reconstructed. The numbers in the right top box are the fraction of correct reconstruction and the RMS of the histogram for all the events (larger histogram). On top from left : samples with zero, $\geq 1$, and two $b$-jets tagged by SECVTX tagger after $\chi^{2}$ cut. On bottom : sample with two $b$-jets tagged by SECVTX and Jet Probability taggers after $\chi^{2}$ cut (left) and $m_{j j}$ cut (right).

where initial or final gluons come in the four leading jets, as well as events that did not even decay into the lepton + jets mode. In fact, we further divide the combinatorial background into more detailed categories to understand the sources of combinatorial background better. We consider the following categories by the type of mis-assignment in the reconstruction ${ }^{1}$ :

a. Correct reconstruction.

b. The case where the $b$-jet from the hadronic top decay is assigned to the $b$-quark from the leptonic top decay, and vice versa. In this case, the jets produced in the hadronic decay of $W$ boson are assigned to the correct partons.

\footnotetext{
${ }^{1}$ In fact, there is a type of mis-assignment that we do not consider here. There is a chance that we flip the assignments of the two jets coming from the hadronic $W$ decay. However, this mis-assignment does not affect the reconstructed top mass in the event. Therefore, we consider an event as correctly reconstructed if this type of mis-assignment is the only mis-assignment in the reconstruction of this event.
} 
c. The case where $c$-jet from the hadronic decay of $W$ boson is assigned to one of the $b$-quarks.

d. Other wrong reconstruction where all the four partons of the $t \bar{t}$ decay come in the leading four jet.

e. Events where gluon-jets come into the four leading jets.

f. Events where the $t \bar{t}$ pair did not decay in the lepton + jets channel.

Table 5.3 summarizes the percentage of events that come into each of these categories for each sample. Besides 0, 1 and 2 tag samples, we present results for several different event categories concerning the tagging conditions.

\begin{tabular}{|c|c|c|c|c|c|c|c|}
\hline \hline & $\begin{array}{c}\text { a. } \\
\text { correct }\end{array}$ & $\begin{array}{c}\text { b. } \\
\text { flipped } \\
b\end{array}$ & $\begin{array}{c}\text { c. } \\
c \text {-jet } \\
\text { to } \\
\end{array}$ & $\begin{array}{c}\text { d. } \\
\text { other } \\
\text { wrong } \\
\text { comb. }\end{array}$ & $\begin{array}{c}\text { e. } \\
\text { assign }\end{array}$ & $\begin{array}{c}\text { f. } \\
\text { other } \\
\text { comb. }\end{array}$ & \\
\hline \hline $2 \mathrm{tag}$ & 50.0 & 14.9 & 2.5 & 5.7 & 17.8 & 9.1 & 3747 \\
$1 \mathrm{tag}$ & 23.9 & 7.2 & 12.9 & 5.4 & 38.4 & 12.2 & 7933 \\
$0 \mathrm{tag}$ & 21.5 & 7.0 & 19.9 & 7.2 & 34.6 & 9.8 & 4935 \\
\hline \hline 2 tag before $m_{j j}$ cut & 44.6 & 13.5 & 3.4 & 7.0 & 21.5 & 10.0 & 4410 \\
2 SECVTX tag & 47.3 & 14.1 & 1.4 & 6.9 & 20.2 & 10.1 & 1979 \\
\hline non-recycled 1 SECVTX tag & 26.4 & 8.0 & 13.1 & 4.7 & 36.2 & 11.7 & 6356 \\
recycled 1 SECVTX tag & 14.1 & 3.8 & 12.1 & 8.1 & 47.5 & 14.5 & 1577 \\
\hline \hline
\end{tabular}

Table 5.3: The percentage (\%) of the events that come into each category of mis-assignment in the event reconstruction. See text for the definition of the categorization. The sum of the six categories is $100 \%$, and the "total" shows the number of Monte Carlo events corresponding to $100 \%$. Besides 0,1 and 2 tag samples, we summarize the results for 2 tag sample without $m_{j j}$ cut and 2 SECVTX tag sample. We also show results for 1 SECVTX tag sample divided into recycled and non-recycled events.

We can retrieve several interesting conclusions from this table. If we compare the percentage of gluon-events (e.) for $1 \mathrm{tag}, 2 \mathrm{tag}$ before $m_{j j}$ and 2 SECVTX tag samples ${ }^{2}$, we see that tightening the $b$-tagging condition helps in reducing the combinatorial background due to the initial or final gluon radiation. We consider this is due to the case where the tightened tagging condition (double $b$-tagging) rejects the event where one of the $b$-jets is not in the leading four jets because a $g$-jet came in instead. Since we have no chance of correctly reconstructing the

\footnotetext{
${ }^{2}$ We can not include the 0 tag channel in this comparison because the kinematic cut is different for this channel.
} 
events in categories e. and f., it would be interesting to see the fraction of events with correct reconstruction with these categories reduced from the denominator. The fraction of events where the choice of four jets match the four partons produced in the lepton + jets decay of the $t \bar{t}$ pair is (addition of a., b., c. and d.) : $73.1 \%, 49.4 \%$ and $55.6 \%$ for 2,1 and 0 tag sample, respectively. If we take the number of such events as the denominator, the fraction of correctly reconstructed events are $68.4 \%, 48.3 \%$ and $38.7 \%$ for 2,1 and 0 tag sample, respectively.

In general, the tagging condition of Jet Probability at JPcut $=0.05$ is looser than that of SECVTX. At this cut, Jet Probability is expected to be efficient in tagging $c$-jets besides $b$-jets. Our concern is whether this property of Jet Probability results in a considerable fraction of combinatorial background of type c. in the 2 tag sample. Due to the table, the fraction of such combinatorial background is only $3.4 \%$ even before $m_{j j}$ cut. At the same time, the small decrease of the fraction of correct reconstruction from 2 SECVTX tag sample, while almost doubling the number of events in $2 \mathrm{tag}$ sample as described above, justifies the use of Jet Probability.

\subsubsection{Dependence of the Event Reconstruction on the Top Quark Mass}

We studied how behavior of our event reconstruction for different top quark masses. We use $t \bar{t}$ Monte Carlo events generated by HERWIG with different top quark mass assumptions.

Figure 5.7 shows how the event selection efficiency for 0,1 and 2 tag sample changes as a function of the top quark mass. Note that the denominator of this efficiency is the total number of generated Monte Carlo events, and the efficiency includes efficiencies of all the applied cuts as well as the branching ratio of $t \bar{t} \rightarrow$ lepton + jets. As the top quark mass gets larger, the final particles (lepton, $E_{T}$ and jets) are produced with higher $P_{T}$ and get better possibility of passing our kinematic cuts. The tight kinematic cut $\left(E_{T}^{4 t h j e t}>21 \mathrm{GeV}\right)$ specially applied for 0 tag sample is considered to be the cause of the different behavior of the 0 tag sample selection efficiency.

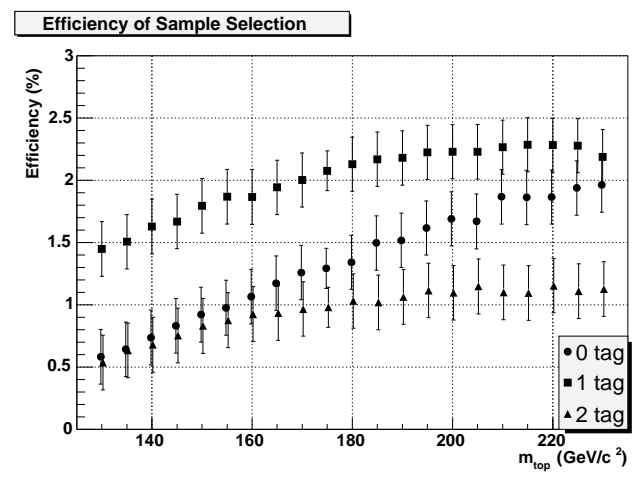

Figure 5.7: Efficiency of our event selection for each sub-sample as a function of the top quark mass. 
Figures 5.8, 5.9 and 5.10 show reconstructed top mass distributions for several different top quark masses for 0,1 and 2 tag samples, and Figure 5.11 summarizes the fraction of correct reconstruction for the three samples as a function of the top quark mass. Here we observe that the fraction of correct combination gets larger as the top quark mass gets larger. We can also see that due to the increase of the correct combination, the width of the reconstructed mass distribution gets narrower as the top quark mass gets larger.
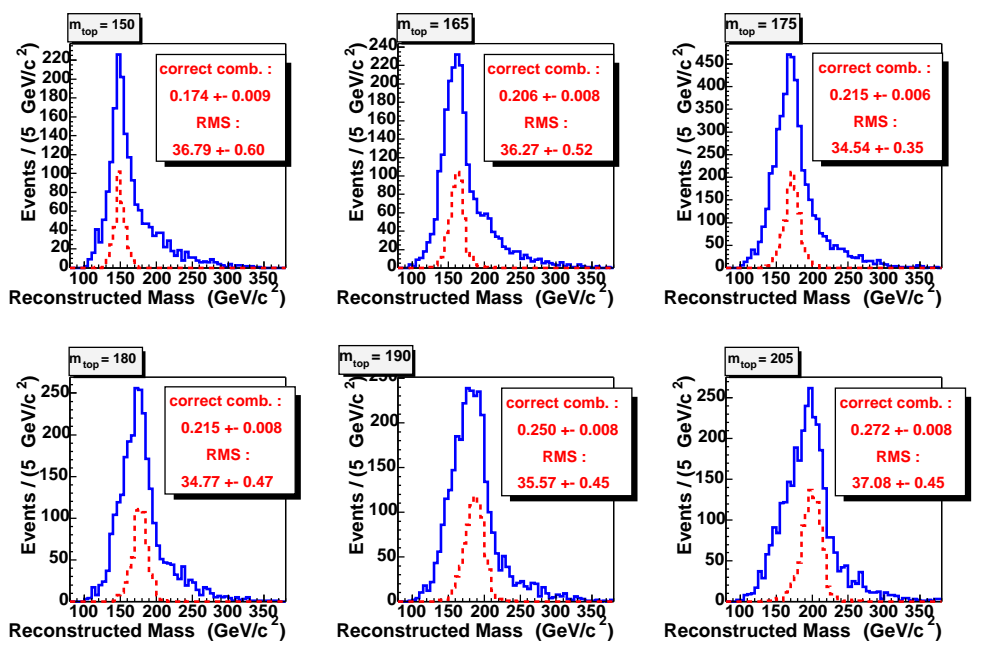

Figure 5.8: Reconstructed top mass distributions for different top quark masses are shown in solid histograms for $0 \mathrm{tag}$ sample. The distributions of the correctly reconstructed events are also shown in dashed histograms. From left on top column : $m_{\text {top }}=150,165$ and $175 \mathrm{GeV} / c^{2}$, from left on bottom column : $m_{\text {top }}=180,190$ and $205 \mathrm{GeV} / c^{2}$. In the top-right box in each plot are the fraction of correct combination, along with the RMS of the solid histogram. 

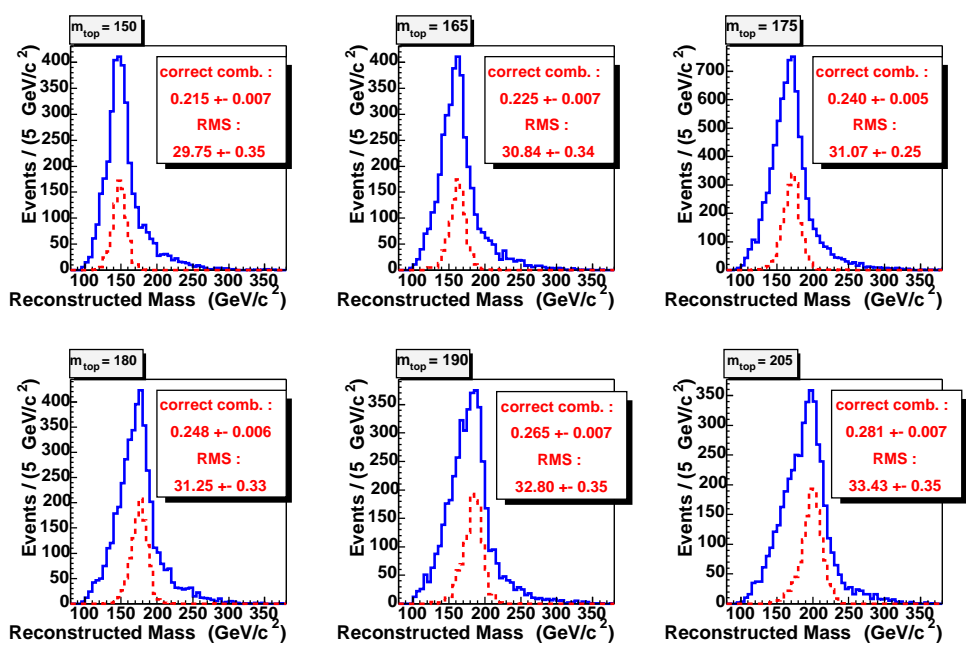

Figure 5.9: Reconstructed top mass distributions for different top quark masses are shown in solid histograms for $1 \mathrm{tag}$ sample. The distributions of the correctly reconstructed events are also shown in dashed histograms. From left on top column : $m_{\text {top }}=150,165$ and $175 \mathrm{GeV} / c^{2}$, from left on bottom column : $m_{\text {top }}=180,190$ and $205 \mathrm{GeV} / c^{2}$. In the top-right box in each plot are the fraction of correct combination, along with the RMS of the solid histogram.
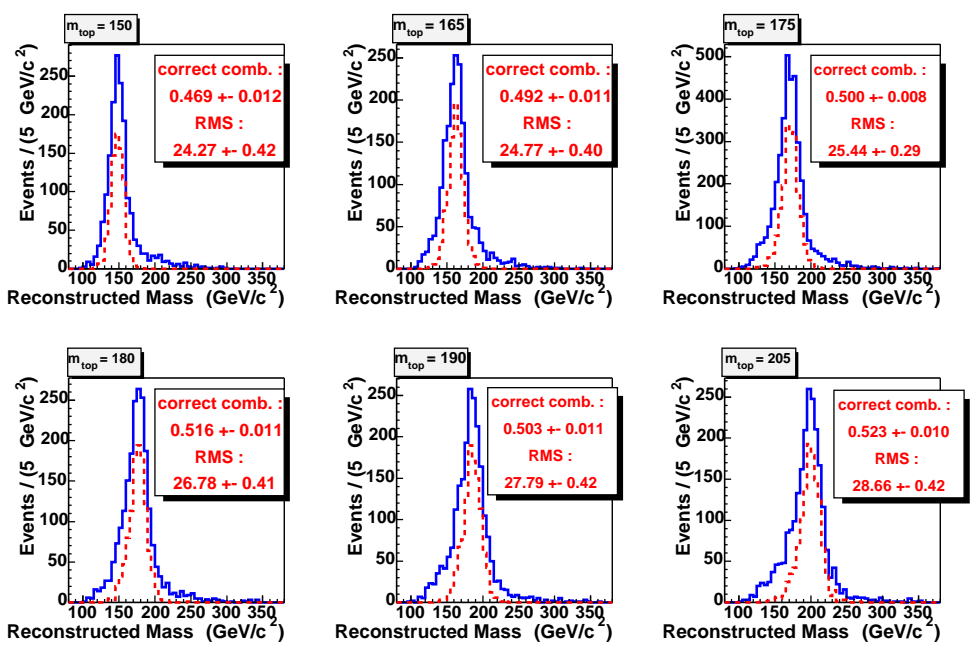

Figure 5.10: Reconstructed top mass distributions for different top quark masses are shown in solid histograms for $2 \mathrm{tag}$ sample. The distributions of the correctly reconstructed events are also shown in dashed histograms. From left on top column : $m_{\text {top }}=150,165$ and $175 \mathrm{GeV} / c^{2}$, from left on bottom column : $m_{\text {top }}=180,190$ and $205 \mathrm{GeV} / c^{2}$. In the top-right box in each plot are the fraction of correct combination, along with the RMS of the solid histogram. 


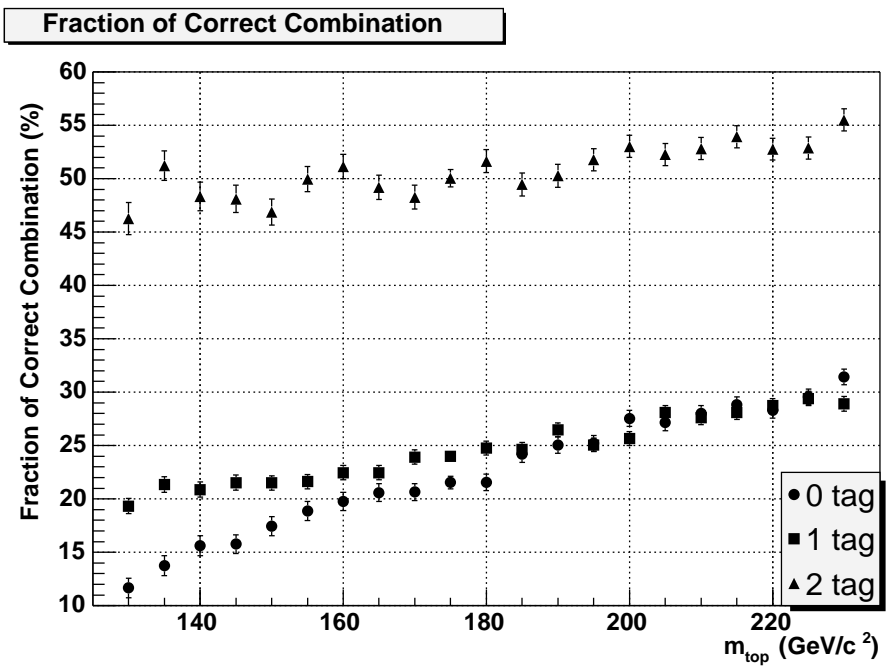

Figure 5.11: The fraction of events with correct reconstruction in each sample is plotted as a function of the top quark mass. 


\section{Chapter 6}

\section{Mass Templates}

In our measurement of the top quark mass, we fit the distribution of the reconstructed top mass extracted from data to a sum of the signal and background distributions derived from Monte Carlo samples. We call these distributions obtained from Monte Carlo studies "templates". The signal shape must be described as a function of the true top mass to perform the top mass measurement. We also fit the background distribution to a certain function. Since we have three independent channels, 0,1 and 2 tag channels, we need to prepare templates for each of them, separately. In this section, we describe the parameterizations of the functions that are adopted to describe the distribution shapes. The templates depend on the cut on Jet Probability, which will be optimized in chapter 8 to 0.05 . In this chapter, we describe the our study on the templates adopting this cut value for Jet Probability.

\section{1 $t \bar{t}$ Signal Templates}

The signal templates are produced by analyzing $t \bar{t}$ Monte Carlo events generated for various top mass inputs. HERWIG Monte Carlo generator was used to generate the Monte Carlo events. We prepared twenty-one Monte Carlo samples generated with the different input masses from $130 \mathrm{GeV}$ to $230 \mathrm{GeV}$ by $5 \mathrm{GeV}$ steps. We parametrize the top mass distribution by the following function ${ }^{1}$.

$$
f_{s}\left(M_{t} \mid m_{t}\right)=p_{8}\left(f_{1}\left(M_{t},\left\{p_{1}, p_{2}, p_{3}\right\}\right)\right)+p_{9}\left(f_{2}\left(M_{t},\left\{p_{4}, p_{5}\right\}\right)\right)+\left(1-p_{8}-p_{9}\right)\left(f_{2}\left(M_{t},\left\{p_{6}, p_{7}\right\}\right)\right)
$$

$$
f_{1}\left(M_{t},\left\{p_{1}, p_{2}, p_{3}\right\}\right)=\frac{p_{3}{ }^{1+p_{2}}}{\Gamma\left(1+p_{2}\right)}\left(M_{t}-p_{1}\right)^{p_{2}} e^{-p_{3}\left(M_{t}-p_{1}\right)}
$$

\footnotetext{
1 We used to parametrize the signal shape with the addition of $f_{1}$ and $f_{2}$, supposing each correspond to combinatorial background and correctly reconstructed events. We further added another $f_{1}$ function in order to improve the $\chi^{2}$.
} 


$$
f_{2}\left(M_{t},\left\{p_{4}, p_{5}\right\}\right)=\frac{1}{\sqrt{2 \pi} p_{5}} e^{-\frac{1}{2}\left(\frac{M_{t}-p_{4}}{p_{5}}\right)^{2}}
$$

where $M_{t}$ refers to the event-by-event reconstructed top mass, and $m_{t}$ is the true top mass. In order for this shape function to have a dependence on $m_{t}$, we define the nine parameters as a linear function of the true top mass.

$$
p_{i}=\alpha_{i}+\alpha_{i+9} \times\left(m_{t}-\left(175 \mathrm{GeV} / c^{2}\right)\right), \quad i=1,2, \ldots, 9
$$

Thus we describe the mass distribution of the signal events with eighteen $\alpha$ parameters plus the true top mass $m_{t}$. The values of the $\alpha$ parameters are determined by fitting the twenty-one signal templates with this function simultaneously. We calculate the $\chi^{2}$ summed over all the twenty-one signal templates and minimize it in order to determine the $\alpha$ parameters. Table 6.1 summarizes the values of $\alpha$ parameters of 0,1 and 2 tag samples as the result of this fitting. The minimized $\chi^{2}$ of 1 tag channel was

$$
\frac{\chi^{2}}{\text { d.o.f. }}=\frac{923.7}{862}
$$

while that of $2 \mathrm{tag}$ was

$$
\frac{\chi^{2}}{\text { d.o.f. }}=\frac{774.5}{697}
$$

and that of the 0 tag sample was

$$
\frac{\chi^{2}}{\text { d.o.f. }}=\frac{1078.4}{878}
$$

We show in Figures 6.1, 6.2 and 6.3 some of the signal templates for 0,1 and 2 tag sample, respectively. Shown along the histograms obtained in Monte Carlo samples are the signal shape functions with $m_{t}$ set to the input top masses used in generating the templates.

\subsection{Background Templates}

The background constitutes of several different physics processes, and we have to build background templates by adding up the distributions from each of them. In this section, we describe our estimation of the number of background events and the template shape, both of which are essential for the top mass measurement as described in section 7. The first half of this section is dedicated to the estimation of the number of background events. In the latter half, we build up our background templates and parametrize them.

\subsubsection{Estimation of the Number of Background Events}

We describe our estimation of the number of events coming from each background process in this subsection. The numbers obtained here will be used in the building up of our background templates as well as for the input parameters of the likelihood fitting described in the next 


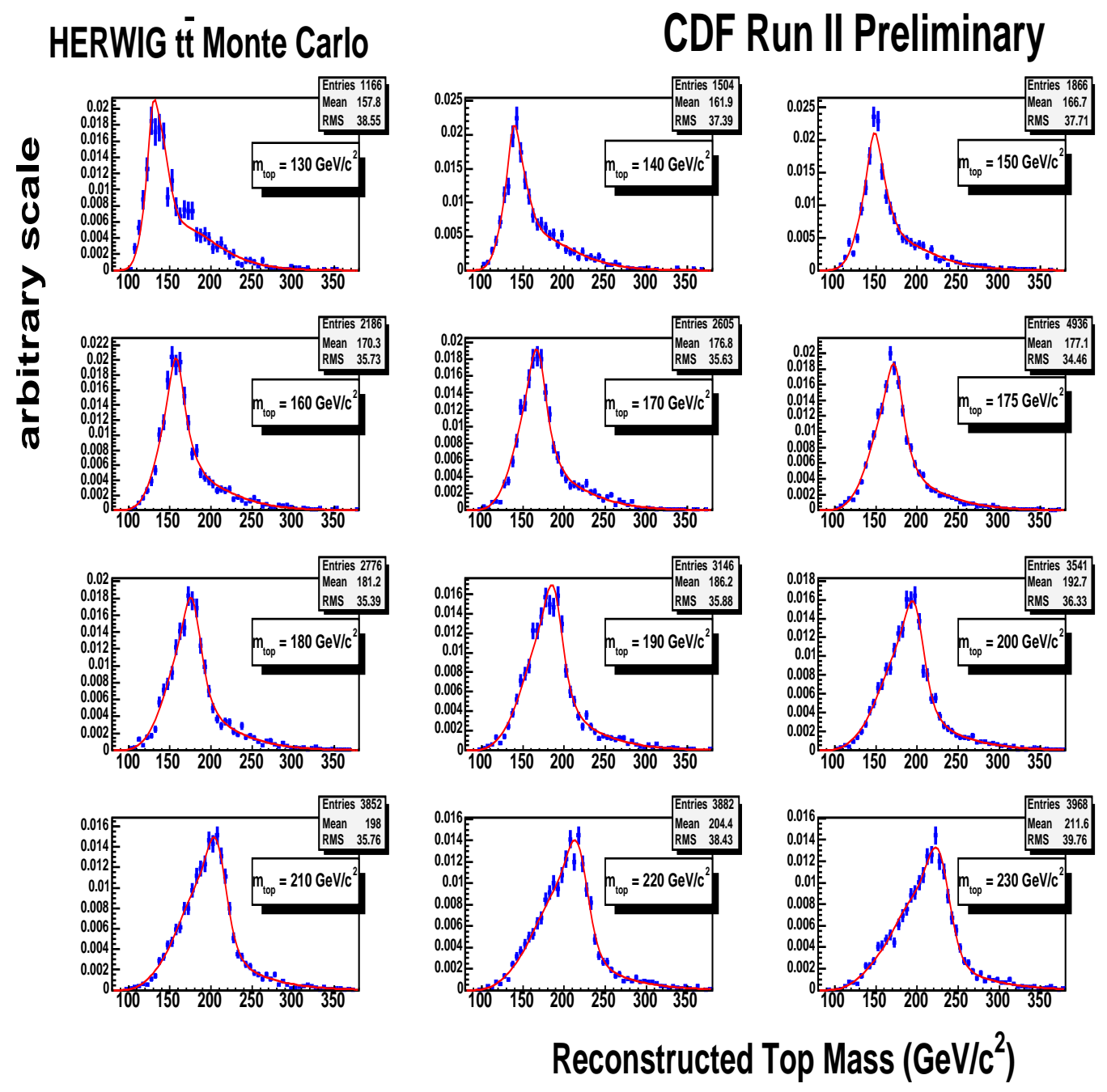

Figure 6.1: Signal templates for the 0 tag sample for input masses from $150 \mathrm{GeV}$ up to $205 \mathrm{GeV}$ along with the parametrized signal shape functions. 


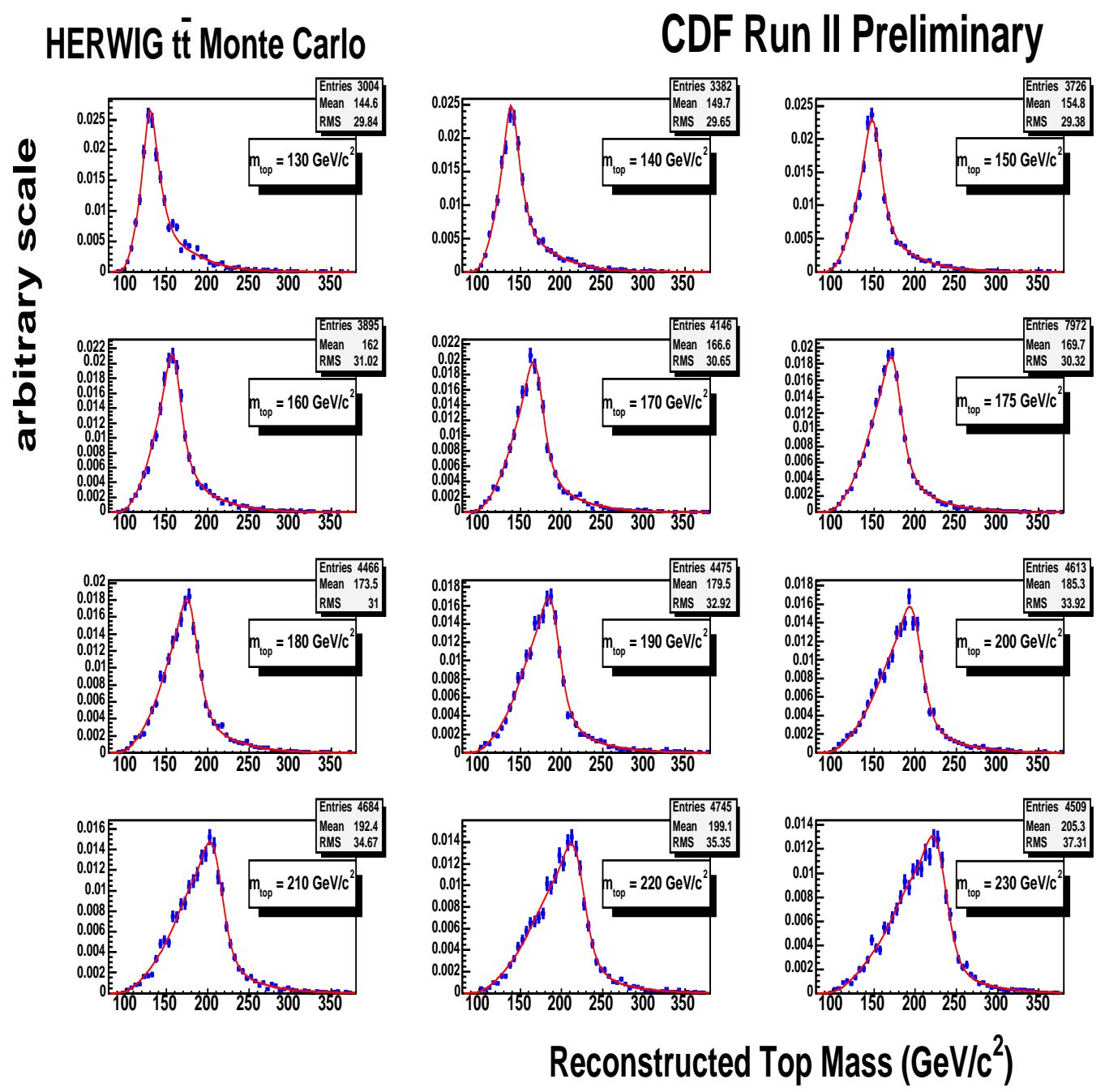

Figure 6.2: Signal templates for the 1 tag sample for input masses from $150 \mathrm{GeV}$ up to $205 \mathrm{GeV}$ along with the parametrized signal shape functions. 


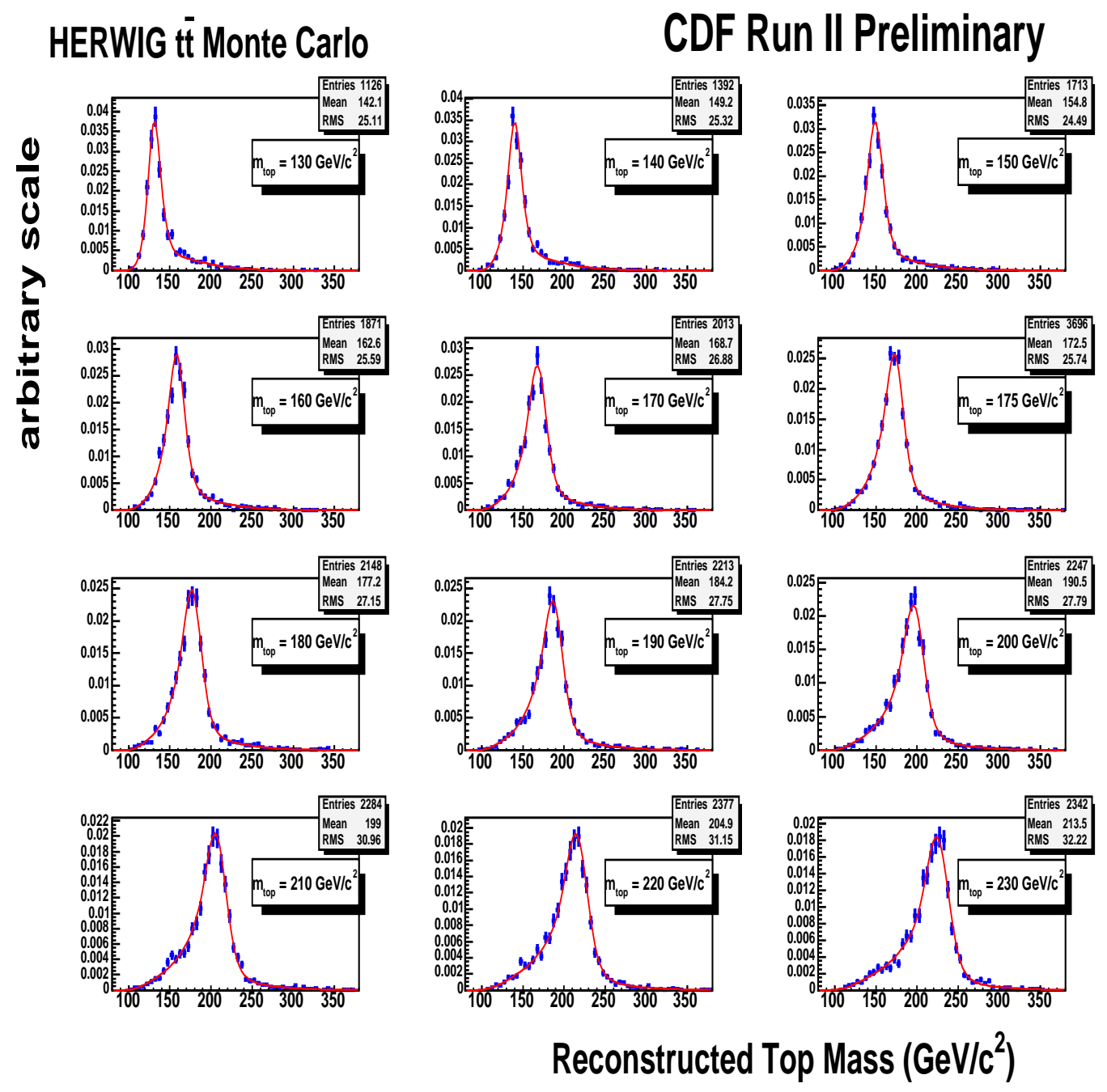

Figure 6.3: Signal templates for the 2 tag sample for input masses from $150 \mathrm{GeV}$ up to $205 \mathrm{GeV}$ along with the parametrized signal shape functions. 


\begin{tabular}{|c|ccc|ccc|ccc|}
\hline \hline$i$ & \multicolumn{3}{|c|}{$\alpha_{i}(0$-tag) } & \multicolumn{3}{c|}{$\alpha_{i}(1$-tag) } & \multicolumn{3}{c|}{$\alpha_{i}(2$-tag) } \\
\hline 1 & 85.4 & \pm & 1.6 & 87.8 & \pm & 0.8 & 92.2 & \pm & 1.3 \\
2 & 4.30 & \pm & 0.22 & 3.25 & \pm & 0.11 & 3.26 & \pm & 0.18 \\
3 & 0.0500 & \pm & 0.0013 & 0.0487 & \pm & 0.0009 & 0.0492 & \pm & 0.0015 \\
4 & 172.8 & \pm & 0.4 & 172.9 & \pm & 0.3 & 173.3 & \pm & 0.3 \\
5 & 8.17 & \pm & 0.56 & 8.44 & \pm & 0.35 & 8.81 & \pm & 0.46 \\
6 & 165.3 & \pm & 0.5 & 164.2 & \pm & 0.4 & 167.6 & \pm & 0.7 \\
7 & 18.5 & \pm & 0.5 & 18.0 & \pm & 0.3 & 16.2 & \pm & 0.8 \\
8 & 0.467 & \pm & 0.013 & 0.461 & \pm & 0.009 & 0.308 & \pm & 0.012 \\
9 & 0.126 & \pm & 0.015 & 0.134 & \pm & 0.011 & 0.308 & \pm & 0.047 \\
10 & -0.0464 & \pm & 0.0509 & -0.1681 & \pm & 0.0271 & -0.1432 & \pm & 0.0648 \\
11 & 0.0002 & \pm & 0.0065 & 0.0261 & \pm & 0.0033 & 0.0188 & \pm & 0.0072 \\
12 & $-1.63 e-04$ & \pm & $3.93 e-05$ & $-2.52 e-05$ & \pm & $2.06 e-05$ & $-7.77 e-05$ & \pm & $4.35 e-05$ \\
13 & 0.971 & \pm & 0.011 & 0.959 & \pm & 0.009 & 0.944 & \pm & 0.008 \\
14 & 0.0882 & \pm & 0.0130 & 0.0697 & \pm & 0.0094 & 0.0721 & \pm & 0.0068 \\
15 & 0.690 & \pm & 0.019 & 0.739 & \pm & 0.013 & 0.783 & \pm & 0.024 \\
16 & 0.142 & \pm & 0.010 & 0.131 & \pm & 0.007 & 0.144 & \pm & 0.012 \\
17 & -0.0009 & \pm & 0.0003 & -0.0007 & \pm & 0.0002 & -0.0003 & \pm & 0.0002 \\
18 & 0.0017 & \pm & 0.0003 & 0.0008 & \pm & 0.0002 & 0.0016 & \pm & 0.0005 \\
\hline \hline
\end{tabular}

Table 6.1: $\quad \alpha$ parameters obtained in the signal template fitting for the 0 (left column), 1 (middle) and 2 (right) tag samples.

chapter. The methods of the estimation are similar between 1 and 2 tag samples. The estimation for the 0 tag sample is calculated in a completely different way, because the dominant background processes differ from the tagged samples.

\section{Non- $W$ Background in 2 Tag Samples}

Our event selection aims to reject events where no $W$ boson exists by requiring cuts on lepton $E_{T}\left(P_{T}\right)$ and missing $E_{T}$. However, events with no $W$ boson production sometimes pass these cuts. We categorize these events as "non- $W$ " process. These events originate in QCD processes and we also call them "QCD background". For example, electrons produced in photon conversions or leptons created in semi-leptonic decays of $B$ hadrons might be identified as leptons. Also there are situations where hadrons are misidentified as leptons. At the same time, large missing $E_{T}$ can occur in situations where jets are mis-measured, or when a portion of a jet lands in a crack between the wedges of the calorimeter. For the estimation of the number of non- $W$ background, we examine the behaviors of $E_{T}$ and lepton isolation of the lepton candidate events in data [59]. The estimation of this background relies on the fact that the isolation of the lepton and $\not_{T}$ are not correlated in non- $W$ process while they are highly correlated in $t \bar{t}$ events. This fact allows us to extrapolate the $\mathbb{E}_{T}$ spectrum of the QCD events in the non-isolated to the 
isolated region. We call this method "isolation vs $E_{T}$ method".

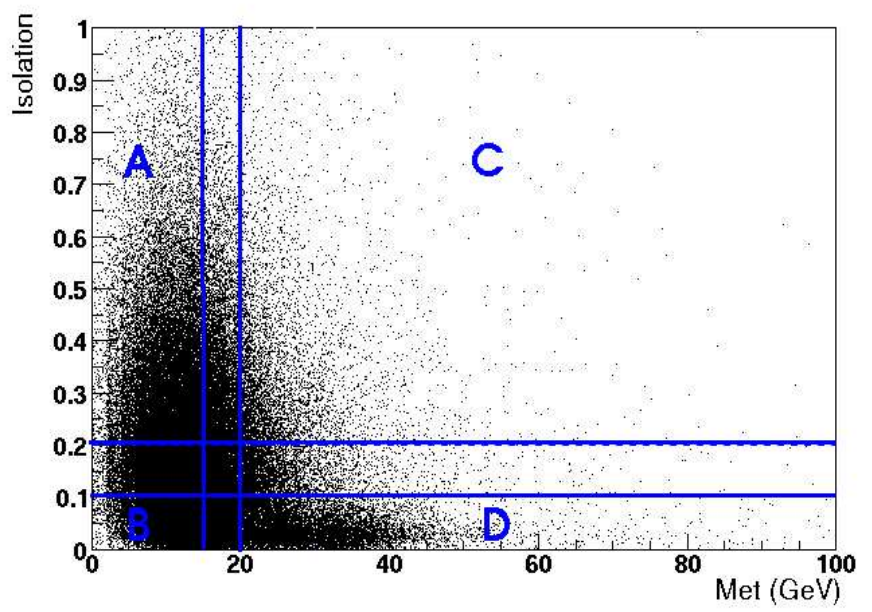

Figure 6.4: Regions are defined in lepton isolation vs $\not_{T}$ plane in order to estimate the non- $W$ background. Region D is the signal region.

The number of events in the following regions are estimated for the non- $W$ background:

- Region $A$ : lepton isolation $>0.2$ and missing $E_{T}<15 \mathrm{GeV}$.

- Region $B$ : lepton isolation $<0.1$ and missing $E_{T}<15 \mathrm{GeV}$.

- Region $C$ : lepton isolation $>0.2$ and missing $E_{T}>20 \mathrm{GeV}$.

- Region $D$ : lepton isolation $<0.1$ and missing $E_{T}>20 \mathrm{GeV}$.

where Region $D$ is the signal region. Figure 6.4 illustrates the definition of these regions as well as a typical distribution of the isolation and $E_{T}$.

Table 6.2 summarizes the number of events in each region before $b$-tag (pretag), after $\geq 1$ SECVTX tag, after $2 \mathrm{tag}$ and after $2 \mathrm{tag}, \chi^{2}$ and $m_{j j}$ cuts. The last category corresponds to our 2 tag sample selection. As discussed above, we suppose that the $\not_{T}$ spectrum is independent of the lepton isolation in non- $W$ events :

$$
\text { non }-W(D)=B \times(C / A)
$$

Since the number of events in region $B$ is 0 , the center value is 0 for the estimated number of non- $W$ background. However, the small statistics after double $b$-tagging would result in a huge error on this estimate. In order to minimize the error on our estimate, we further assume that 


\begin{tabular}{|c|c|c|c|c|c|c|}
\hline & lepton & jet multiplicity & region A & region B & region C & region D \\
\hline pretag & electron & $W+3.5 \mathrm{j}$ & 418 & 134 & 47 & 78 \\
& muon & $W+3.5 \mathrm{j}$ & 199 & 23 & 31 & 60 \\
& electron & $W+4 \mathrm{j}$ & 130 & 65 & 33 & 69 \\
& muon & $W+4 \mathrm{j}$ & 53 & 11 & 22 & 35 \\
\hline$\geq 1$ & electron & $W+3.5 \mathrm{j}$ & 23 & 6 & 10 & 4 \\
SECVTX & muon & $W+3.5 \mathrm{j}$ & 27 & 2 & 8 & 6 \\
tag & electron & $W+4 \mathrm{j}$ & 5 & 7 & 4 & 16 \\
& muon & $W+4 \mathrm{j}$ & 6 & 3 & 10 & 11 \\
\hline 2 tag & electron & $W+3.5 \mathrm{j}$ & 1 & 2 & 1 & 2 \\
& muon & $W+3.5 \mathrm{j}$ & 0 & 1 & 1 & 6 \\
& electron & $W+4 \mathrm{j}$ & 1 & 0 & 0 & 1 \\
& muon & $W+4 \mathrm{j}$ & 0 & 0 & 3 & 6 \\
\hline 2 tag & electron & $W+3.5 \mathrm{j}$ & 0 & 0 & 0 & 4 \\
after & muon & $W+3.5 \mathrm{j}$ & 1 & 0 & 0 & 0 \\
$m_{j j}$ & electron & $W+4 \mathrm{j}$ & 0 & 0 & 0 & 4 \\
cut & muon & $W+4 \mathrm{j}$ & 0 & 0 & 2 & 5 \\
\hline \hline
\end{tabular}

Table 6.2: The number of events in the regions defined above for before $b$-tag, $\geq 1$ SECVTX tag, 2 tag and 2 tag after $\chi^{2}$ and $m_{j j}$ cuts. 
tagging conditions do not affect the ratio $C / A$ :

$$
(C / A)_{2 t a g}=(C / A)_{p r e t a g}
$$

We assign the error of 1.14, the Poisson upper limit for 0 at $68 \%$ confidence level, for $B=0$. Thus, we obtain the estimate of $0_{-0}^{+0.19}$ events for non- $W$ background in the 2 tag sample. The number of non- $W$ background with $\geq 1$ SECVTX tag in the event is also calculated in the same method, without using the assumption of Equation 6.6, which is presented in Table 6.3 with estimates for other background sources ${ }^{2}$.

\begin{tabular}{|c|c|c|}
\hline \hline Source & $W+3.5 \mathrm{jets}$ & $W+4$ jets \\
\hline non- $W$ (QCD) & $1.7 \pm 0.5$ & $1.7 \pm 0.5$ \\
$W+$ jets (mistag) & $1.6 \pm 0.3$ & $1.2 \pm 0.3$ \\
$W b \bar{b}$ & $1.0 \pm 0.4$ & $0.6 \pm 0.3$ \\
$W c \bar{c}$ & $0.4 \pm 0.2$ & $0.2 \pm 0.1$ \\
$W c$ & $0.5 \pm 0.2$ & $0.2 \pm 0.1$ \\
single top (s-chan.) & $0.18 \pm 0.03$ & $0.13 \pm 0.02$ \\
single top (t-chan.) & $0.12 \pm 0.02$ & $0.06 \pm 0.02$ \\
$W W / W Z$ & $0.17 \pm 0.03$ & $0.12 \pm 0.02$ \\
\hline total & $5.7 \pm 0.8$ & $4.4 \pm 0.7$ \\
\hline \hline
\end{tabular}

Table 6.3: Estimated number of background with $\geq 1$ SECVTX $b$-tag required. These are the numbers before $\chi^{2}$ cut.

\footnotetext{
${ }^{2}$ Since the jet bins of concern contain $t \bar{t}$ process in data, we have to subtract its contribution. All the background estimates adopting normalization by data - non- $W, W+$ heavy flavor and $\operatorname{mistag}(W+\operatorname{light}$ flavor $)$ are subject to this correction. All the numbers we mention in this section are those after accounting for the $t \bar{t}$ contribution.

This correction is taken care of as in the following. The cross section for the $t \bar{t}$ creation process $\sigma_{t \bar{t}}$ is measured using the $\geq 1$ SECVTX sample with formula :$$
\sigma_{t \bar{t}}=\frac{N_{\text {observed }}-N_{\text {background }}}{\varepsilon_{t \bar{t}} \times L}
$$

where $N_{\text {observed }}$ is the number of events observed in data, $N_{\text {background }}$ is the number of background events estimated in the way described in this section. $\varepsilon_{t \bar{t}}$ refers to the total signal acceptance of the analysis including the $b$-tagging efficiency and $L=162 \mathrm{pb}^{-1}$ is the integrated luminosity used in the analysis. The number of estimated background events is expanded :

$$
N_{\text {background }}=N_{\text {non }-W}+N_{W+H F}+N_{\text {mistag }}+N_{\text {otherbackgrounds }}
$$

and $N_{n o n-W}, N_{W+H F}$ and $N_{\text {mistag }}$ are corrected for, i.e. functions of, $\sigma_{t \bar{t}}$. The $t \bar{t}$ cross section is measured by iteratively calculating Eqn 6.7 with inputing different values of $\sigma_{t \bar{t}}$ on the right side until $\sigma_{t \bar{t}}$ on both sides become equal. The $t \bar{t}$ contribution [60] was accounted for using $\sigma_{t \bar{t}}=5.6 \pm 1.2 \pm 0.9 \mathrm{pb}^{-1}$ obtained in this way.
} 


\section{$W+$ Heavy Flavor Background in 2 Tag Sample}

$W+$ heavy flavor backgrounds are the events which contain a real $W$ boson associated with heavy flavor (bottom or charm) partons of $Q C D$ production. There are three such backgrounds to the $t \bar{t}$ sample : $W b \bar{b}, W c \bar{c}$ and $W c$.

Figure 6.5 shows an example of $W b \bar{b}$ production process where a $b \bar{b}$ pair is created due to gluon splitting. More complicated diagrams contribute to the production of this background process. Figure 6.5 is one of such diagrams. Especially because gluons couple strongly, the correction of the higher order is not negligible and the addition of the higher orders is a complicated procedure. In fact, next-to-leading order corrections are at present known only for the $W+0$ and 1 jet production cross sections [6]. Therefore, it is very difficult to precisely calculate an absolute cross section for this background process $(W+4$ jet processes) and we can not use a calculated theoretical cross section in our estimation.

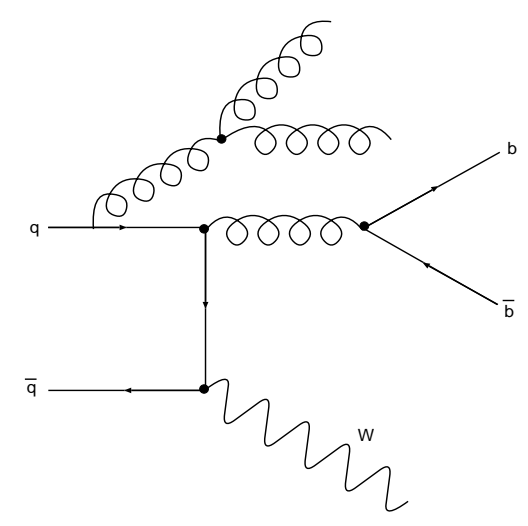

Figure 6.5: One of the production processes for $W b \bar{b}$ in the leading order.

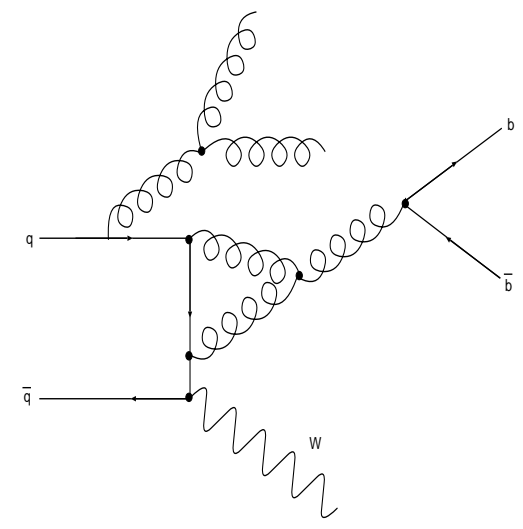

Figure 6.6: One of the production processes for $W b \bar{b}$ in the next to leading order.

However, the ratio of $W+$ heavy flavor and inclusive $W+$ jets events in each jet bin should 
cancel these complications, and can be derived from studies on the Monte Carlo samples of the leading order calculation. In this way, the number of the $W+$ heavy flavor background $N_{H F}$ after requiring $\geq 1$ SECVTX tag can be estimated as follows :

$$
N_{W+H F}=N_{W} \times\left(1-F_{n o n-W}\right) \times F_{H F} \times \varepsilon_{t a g} \times S F_{t a g}
$$

where $N_{W}$ is the number of $W+$ jets candidates (including non- $W$ ) in the jet bin of concern before tagging, $F_{n o n-W}$ is the fraction of the non- $W$ background in the jet bin, $F_{H F}$ is the fraction of number of $W+$ heavy flavor events in true $W+$ jets events, $\varepsilon_{t a g}$ is the $b$-tagging efficiency for the $W+$ heavy flavor events and $S F_{t a g}$ is the data-to-Monte Carlo scale factor for the $b$-tagging. $F_{n o n-W}$ is calculated using the "isolation vs $E_{T}$ " method we described in the previous section. $F_{H F}$ is derived from studies with $W+$ jets sample generated by ALPGEN Monte Carlo generator, which incorporates the calculation of the physics process in the leading order. $\varepsilon_{t a g}$ is also calculated using the same Monte Carlo sample. Number of estimated $W b \bar{b}$, $W c \bar{c}$ and $W c$ backgrounds after requiring $\geq 1$ SECVTX tag is summarized in Table 6.3 along with other background sources.

We further calculated the efficiencies of our secondary tag (number of double tagged events divided by number of $\geq 1$ SECVTX tagged events), our $\chi^{2}$ and $m_{j j}$ cuts by using the ALPGEN Monte Carlo sample. By multiplying these efficiencies by the number with $\geq 1$ SECVTX tag, we obtain the expected number of $W+$ heavy flavor in the 2 tag sample. The efficiency values and the estimated number of events for $W b \bar{b}, W c \bar{c}$ and $W c$ backgrounds are summarized in Table 6.4 along with estimates for other background processes.

\begin{tabular}{|c|c|c|c|c|c|c|}
\hline \hline process & $N_{162 \mathrm{pb}^{-1}}^{\geq 1 \text { tag }}$ & $\varepsilon_{2 \text { ndtag }}(\%)$ & $\varepsilon_{\chi^{2}}(\%)$ & $\varepsilon_{m_{j j}}(\%)$ & $\varepsilon_{\text {total }}(\%)$ & $N_{162 \mathrm{pb}-1}^{2 \text { tag }}$ \\
\hline non- $W$ & $1.7 \pm 0.5$ & - & - & - & - & $0_{-0}^{+0.19}$ \\
mistag & $2.8 \pm 0.5$ & - & - & - & - & $0.066 \pm 0.008$ \\
Wbb & $1.6 \pm 0.5$ & $30.71 \pm 1.19$ & $37.61 \pm 2.26$ & $67.05 \pm 3.57$ & $7.74 \pm 0.69$ & $0.13 \pm 0.05$ \\
Wcc & $0.6 \pm 0.5$ & $14.35 \pm 1.21$ & $28.93 \pm 4.12$ & $85.71 \pm 5.91$ & $3.56 \pm 0.64$ & $0.02 \pm 0.01$ \\
Wc & $0.7 \pm 0.2$ & $9.36 \pm 1.29$ & $29.17 \pm 6.56$ & $100.00{ }_{-8.14}^{+0}$ & $2.73 \pm 0.72$ & $0.02 \pm 0.02$ \\
single top (s) & $0.31 \pm 0.06$ & $46.63 \pm 1.38$ & $35.96 \pm 1.94$ & $71.23 \pm 3.05$ & $11.94 \pm 0.90$ & $0.037 \pm 0.008$ \\
single top (t) & $0.18 \pm 0.03$ & $19.29 \pm 2.15$ & $47.69 \pm 6.19$ & $64.52 \pm 8.59$ & $5.93 \pm 1.29$ & $0.011 \pm 0.004$ \\
\hline \hline total & $10.1 \pm 1.1$ & - & - & - & - & $0.28_{-0.05}^{+0.20}$ \\
\hline
\end{tabular}

Table 6.4: Summary of the background estimation for $2 \mathrm{tag}$ sample. $N_{162}^{\geq 1 \mathrm{tag} b^{-1}}$ is the sum of numbers of $W+3.5$ jets and $W+4$ jets in Table 6.3. $\varepsilon_{2 n d t a g}, \varepsilon_{\chi^{2} c u t}$ and $\varepsilon_{m_{j j}}$ are efficiencies for our secondary tag, $\chi^{2}$ cut and $m_{j j}$ cut respectively. $\varepsilon_{\text {total }}$ refers to the total efficiency of these three cuts. Errors on these four efficiencies come purely from Monte Carlo statistics. Multiplying $N_{162 \mathrm{pb}^{-1}}^{\geq 1 \mathrm{tag}}$ by $\varepsilon_{\text {total }}$, we obtain the estimated number of background events in 2 tag sample at an integrated luminosity of $162 \mathrm{pb}^{-1} \cdot \varepsilon_{m_{j j}}$ is $100 \%$ for $W c$ Monte Carlo sample because no event was rejected at this cut due to low statistics. The error on this $\left({ }_{-8.14}^{+0}\right)$ comes from the Poisson statistics. 
A concern here is why $W b \bar{b}$ and $W c \bar{c}$ have different efficiencies for our cuts. Since the tagging efficiency is smaller for $c$-jets than for $b$-jets, we expect more events with light flavor mistagged jet in $W c \bar{c}$. In such cases, $c$-jets untagged would be corrected with the top specific correction for the light flavor jets, which results in lower $P_{T}$ for the $c$-jets in the event reconstruction. We suspect this is the reason we have different efficiencies for $W b b$ and $W c c$. To illustrate, we plotted $m_{j j}$ after the $\chi^{2}$ cut for the background processes in Figure 6.7. In the case of $W c \bar{c}$, we more often calculate $m_{j j}$ with a $c$-jet and and a light flavor jet than in $W b \bar{b}$ case. The $c$-jet is measured to have lower $P_{T}$ than reality as described above. Supposing that the distribution of the parton $P_{T}$ is common between different flavors in $W+$ jets processes, we expect $m_{j j}$ to be distributed in lower region in $W c \bar{c}$ than in $W b \bar{b}$, which agrees with the plot in Figure 6.7.
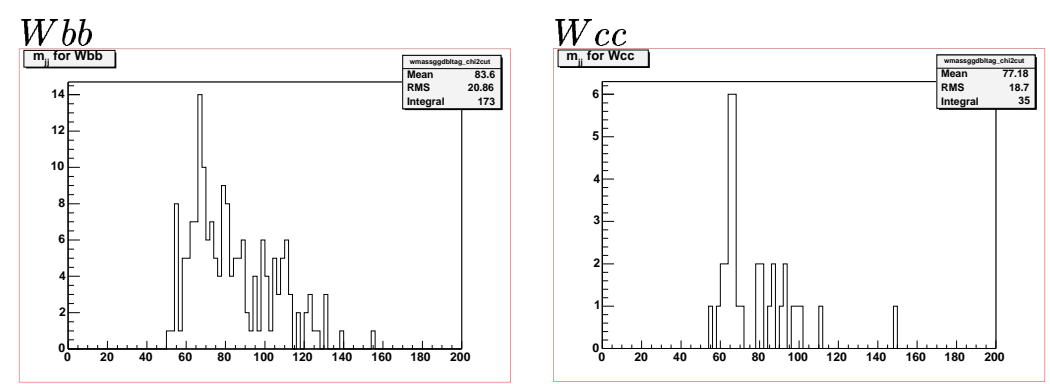

Figure 6.7: $m_{j j}$ distributions for $W b \bar{b}$ (left) and $W c \bar{c}$ (right).

\section{Mistag Background in 2 Tag Sample}

Although we apply $b$-tagging for this sample, $W+$ light flavor jets events sometimes pass our $b$-tagging condition. This is because the $b$-tagging algorithms sometimes mis-tag the light flavor jets due to mis-measurements of tracks. For this reason, we call this background source "mistag background". Since this background process has a huge production cross section, it could be one of our dominant background sources although the tagging algorithms are designed so that the mistag rate is suppressed.

The $b$-tagging algorithms require cuts $L_{x y} / \sigma_{L_{x y}}>3$ (SECVTX) and JetProbability $<J P$ cut (Jet Probability). These cuts are located at around the typical values that heavy flavor jets take for these variables, which enables us to compensate for the difference between data and Monte Carlo by simply introducing the $b$-tagging scale factors. On the other hand, these cuts are placed at the far tail regions of the distributions for light flavor jets. The behavior of the $b$-tagging algorithms is, therefore, highly affected by the slight difference of the distributions between data and Monte Carlo. For this reason, simulating the $b$-tagging algorithms with light flavor jets in Monte Carlo events does not reliably reproduce their behavior in data. We can not simply apply the tagging algorithms to the light flavor jets in the Monte Carlo samples to estimate the number of $W+$ jets events that pass our tagging conditions. We instead use the tagging rate of 
the $b$-taggers for light flavor jets derived from real collision data in the estimation of the number of this background.

The mistag rate - in other words, the efficiencies of the b-tagging algorithms to tag light flavor jets - of SECVTX and Jet Probability taggers have been parametrized as functions of the properties of the jet and the event the jet is in. The mistag rate for SECVTX is parametrized as a function of the following five variables : $E_{T}, \eta, \phi$ and the track multiplicity of the jet, and the sum of the $E_{T}$ of all the jets with $E_{T}>10 \mathrm{GeV}$ and $\mid$ eta $\mid<2.4$ in the event. On the other hand, the mistag rate for Jet Probability tagging is parametrized as a function of the following six variables : $E_{T}, \eta, \phi$ and the track multiplicity of the jet, the $z$ coordinate of the primary vertex, and the sum of the $E_{T}$ of all the jets with $E_{T}>8 \mathrm{GeV}$ and $|e t a|<2.4$ in the event. In either case, the five (or six) variables are binned and the mistag rate is calculated for each bin in the five (six) dimensional space [61] [45]. "Mistag matrix" refers to this five (six) dimensional table of mistag rates. The mistag matrix was derived from collision data independent of our analysis sample.

The mistag background is estimated by applying the mistag matrix to the data events passing through our kinematic cuts. For each event, the probability of the event passing through the cuts for 2 tag sample is calculated by using mistag matrix supposing all the jets in the event is of light flavor. We obtain the estimated number of mistag background events in the analysis sample by summing up such probability over all the events in the data sample, with the non- $W$ contribution to the data sample subtracted.

The estimation of mistag background in $\geq 1$ SECVTX sample is done simply considering the mistag rate of the SECVTX tagger. The result is presented in Table 6.3.

The case of $2 \mathrm{tag}$ sample is more complicated. The two taggers are expected to be correlated to each other although the correlation is not evaluated. We require one SECVTX jet, and allow Jet Probability tagging along with SECVTX for the secondary $b$-tagging. Considering both mistag rates for SECVTX and Jet Probability for the second tagging corresponds to the situation where these taggers are completely independent to each other, and would result in an overestimate of the background rate.

$$
\text { (a) } S E C V T X(-) * S E C V T X(-)+S E C V T X(-) * J P(-)+J P(-) * S E C V T X(-)
$$

where $S E C V T X(-)$ and $J P(-)$ refer to the mistag rates of SECVTX and Jet Probability algorithms, respectively ${ }^{3}$. On the other hand, only taking account the mistag rate for Jet Probability for the second $b$-tagging - the mistag rate is larger for Jet Probability, and this scenario corresponds to the case where the two taggers are completely correlated - would result

\footnotetext{
${ }^{3}$ In the case of SECVTX, the tagging is performed by applying a cut on $L_{X Y} / \sigma_{L_{X Y}}$ of the secondary vertex in relation to the primary vertex. In an ideal case, the light flavor jet originates in the primary vertex and this variable would be always 0 . This variable, however, would have a certain distribution due to the resolution of the detector. The distribution on the positive side is skewed by the heavy flavor jets, but the negative part of the distribution only reflects the detector resolution. Based on this discussion, the mistag rate is measured using the negative part of the $L_{X Y} / \sigma_{L_{X Y}}$ distribution. Similarly, the mistag rate of Jet Probability is measured only using tracks with negative sign impact parameters in the jet. We denote mistag rates with (-) for this reason.
} 
in an underestimate of the background rate.

(b) $S E C V T X(-) * J P(-)+J P(-) * S E C V T X(-)$

We make estimations with both of these assumptions, and take the difference as the uncertainty of our estimate.

Estimated number of events for the item (a) is $0.0663 \pm 0.0052$, on the other hand, for item (b) is $0.0606 \pm 0.0049$. In this mistag study, we used the overestimated background rate and the difference of the above two ways was regarded as the systematic uncertainty. Thus, our estimate for the contribution from the mis-tag background is $0.066 \pm 0.008$ events.

\section{Single Top and Di-Boson Processes in 2 Tag Sample}

In addition to the $t \bar{t}$ pair creation which is the focus of this thesis, top quarks can also be produced singly, in association with a $b$ quark. Observation of such production of top quarks has not been established yet, but it is predicted by the Standard Model and could be a substantial source of background. The single top quark production occurs via electroweak interactions. There are two such processes at the Tevatron, through either the off-mass shell $W$ production ( $s$-channel) and through $W$-gluon fusion ( $t$-channel) as illustrated in Figure 6.8.

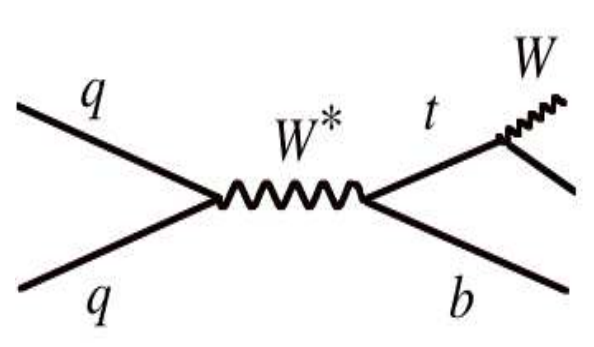

S-channel production of single top $\left(\mathrm{W}^{\star}\right)$

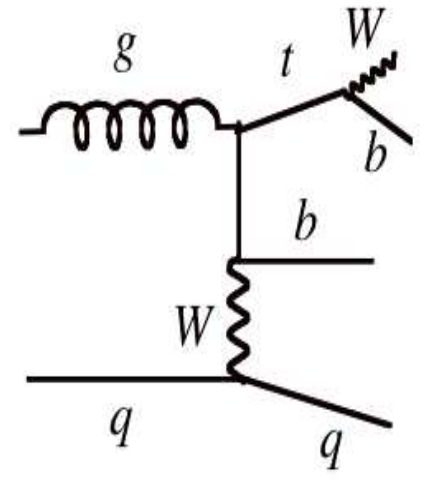

t-channel production of single top $(\mathrm{W}-\mathrm{g})$

Figure 6.8: Diagrams of single top production at Tevatron. Off-mass shell $W$ production (left) and $W$-gluon fusion (right).

The cross section calculations of the electroweak processes are much more precise and trustworthy than those of QCD processes. This allows us to calculate the expected contribution of the single top background in a straightforward fashion, normalizing the integrated luminosity of our data to the theoretical cross section. We estimate the number of single top background events $N_{b k g d}$ in our samples using this formula :

$$
N_{b k g d}=\left(S F \cdot \varepsilon_{t a g}\right) \times\left(\sigma \cdot L \cdot \varepsilon_{a c c}\right)
$$


where $\sigma$ is the theoretically calculated cross section $(0.884 \pm 0.004$ (stat) \pm 0.050 (syst) pb for $s$-channel and $1.980 \pm 0.004$ (stat) \pm 0.113 (syst) pb for $t$-channel. Both calculations are of nextto-leading order (NLO) [62].). $L=162 \mathrm{pb}^{-1}$ is the integrated luminosity of our data sample, $\varepsilon_{a c c}$ is the kinematic acceptance of our analysis, $\varepsilon_{t a g}$ and $S F$ are the double $b$-tagging efficiency for single top events and the scale factor corresponding to it. $\varepsilon_{a c c}$ and $\varepsilon_{t a g}$ are estimated in a Monte Carlo study using single top Monte Carlo samples generated with PYTHIA. $\varepsilon_{a c c}$ is further corrected for the data-to-Monte Carlo scale factor for lepton identification efficiency, and is multiplied by the efficiencies of trigger and primary vertex cut $\left(\left|z_{0}\right|<60 \mathrm{~cm}\right)$ which our Monte Carlo studies do not simulate.

Result of the estimation for $\geq 1$ SECVTX tag sample is shown in Table 6.3. We further calculated the efficiencies of our secondary tag (number of double tagged events divided by number of $\geq 1$ SECVTX tagged events), our $\chi^{2}$ and $m_{j j}$ cuts by using the Monte Carlo sample. By multiplying these efficiencies to the number with $\geq 1$ SECVTX tag, we obtain the expected number of single top events in the 2 tag sample (Table 6.4).

Di-boson production $(W W / W Z)$ also occur in electroweak and are expected to contribute as the background in our analysis. The estimation is performed in a very similar way to the single top background, using Equation 6.9. A NLO theoretical cross section in [63] is used. Monte Carlo samples generated by ALPGEN generator were used in the acceptance and $b$-tagging efficiency studies. Result of Table the estimation for $\geq 1$ SECVTX tag sample is shown in Table 6.3. As for the $2 \mathrm{tag}$ sample, we assume these processes are negligible, and do not perform the estimation.

\section{Estimation of Number of Background Events for 1 Tag Sample}

The estimation of the number of background events in $1 \mathrm{tag}$ sample is performed in a very similar manner to the case of 2 tag sample. The basic strategy is also to calculate the $\chi^{2}$ cut efficiency for each process using Monte Carlo samples, and multiply it to the the number in Table 6.3. For simplicity of the calculation, we estimate the number of background events passing through the $\chi^{2}$ cut for $\geq 1$ SECVTX tag. As was noted in Section 5.3, the number of events in 1 tag sample can be calculated by subtracting the number of events in 2 tag sample from that with $\geq 1$ SECVTX tag. We calculated the efficiencies separately for the 3.5 and 4 jet bins for the $\geq 1$ SECVTX tag case. Another difference is that we merged the mistag and non- $W$ backgrounds, and $W+$ heavy flavor processes and di-boson processes in the calculation of the $\chi^{2}$ efficiency instead of performing the estimation of $\chi^{2}$ cut efficiency separately. $W b \bar{b}$ Monte Carlo sample was used in the estimation of the $\chi^{2}$ efficiency for $W+$ heavy flavor and di-boson processes. The $\chi^{2}$ cut efficiency for mistag and non- $W$ processes was estimated applying the mistag matrix to $W+$ light flavor jets Monte Carlo sample. The calculated $\chi^{2}$ cut efficiencies and the estimated numbers of events from each background source is summarized in Tables 6.5 (for 3.5 jet bin) and 6.6 (for 4 jet bin). 


\begin{tabular}{|c|c||c|c|c|}
\hline \hline Mass Template Source & Background Source & $N_{162 \mathrm{pb}^{-1} \geq 1 \text { tag }}$ & $\varepsilon_{\chi^{2}<9}(\%)$ & $N_{162 \mathrm{pb}^{-1}}^{\geq 1 \text { tag }}$ \\
\hline mistag & mistag, non- $W$ & $3.3 \pm 0.6$ & $71.6 \pm 7.0$ & $2.4 \pm 0.5$ \\
$W+$ heavy flavor & $W b \bar{b}, W c \bar{c}, W c, W W / W Z$ & $2.1 \pm 0.6$ & $66.8 \pm 1.1$ & $1.4 \pm 0.4$ \\
single top ( $s$-chan.) & & $0.18 \pm 0.03$ & $59.8 \pm 1.6$ & $0.11 \pm 0.02$ \\
single top (t-chan.) & & $0.12 \pm 0.02$ & $79.3 \pm 2.6$ & $0.10 \pm 0.01$ \\
\hline total & & $5.7 \pm 0.8$ & - & $4.0 \pm 0.6$ \\
\hline \hline
\end{tabular}

Table 6.5: The estimation of the number of $\geq 1$ SECVTX tagged background events passing through $\chi^{2}<9$ cut in the 3.5 jet bin.

\begin{tabular}{|c|c||c|c|c|}
\hline \hline Mass Template Source & Background Source & $N_{162 \mathrm{pb}^{-1 \geq 1 \text { tag }}}$ & $\varepsilon_{\chi^{2}<9}(\%)$ & $N_{162 \mathrm{pb}^{-1}}^{\geq 1 \text { tag, }}$ \\
\hline mistag & mistag, non- $W$ & $2.9 \pm 0.6$ & $70.7 \pm 7.0$ & $2.0 \pm 0.5$ \\
$W+$ heavy flavor & $W b \bar{b}, W c \bar{c}, W c, W W / W Z$ & $1.1 \pm 0.6$ & $69.2 \pm 1.2$ & $0.7 \pm 0.3$ \\
single top ( $s$-chan.) & & $0.13 \pm 0.02$ & $66.5 \pm 1.8$ & $0.09 \pm 0.01$ \\
single top ( $t$-chan.) & & $0.06 \pm 0.02$ & $56.4 \pm 4.0$ & $0.03 \pm 0.01$ \\
\hline total & & $4.4 \pm 0.7$ & - & $2.9 \pm 0.5$ \\
\hline \hline
\end{tabular}

Table 6.6: The estimation of the number of $\geq 1$ SECVTX tagged background events passing through $\chi^{2}<9$ cut in the 4 jet bin.

As mentioned above, the number of events in 1 tag sample can be calculated by subtracting the number of events in 2 tag sample from that with $\geq 1$ SECVTX tag, which has been estimated in this subsection. Summarized in the " 1 tag" row of Table 6.7 is the 1 tag background estimation after performing this subtraction.

\section{Estimation of Number of Background Events for 0 Tag Sample}

The estimation of the number of background events in 0 tag sample is performed in a different way than the tagged samples. We rather estimate the number of signal events from the number of estimated background events in the $\geq 1$ SECVTX events, then subtract it from the total number of 0 tag events observed in data in order to obtain the estimated number of backgrounds in this sample.

The number of $\geq 1$ SECVTX events in the 4 jet bin found in the $162 \mathrm{pb}^{-1}$ data taken with good silicon operation condition was $N_{t a g}^{162 \mathrm{pb}^{-1}}=27$. As in Table $6.3, N_{b g}^{162 \mathrm{pb}^{-1}}=4.4 \pm 0.7$ events of backgrounds are estimated to be contained in this 27 events. The efficiency to tag at least one jet by SECVTX in $t \bar{t}$ events is calculated : $\varepsilon_{t a g}=0.523 \pm 0.003$ (stat) \pm 0.037 (syst). Using these numbers, the number of $t \bar{t}$ events in $162 \mathrm{pb}^{-1}$ data before requiring the tagging 
$N_{\text {sig,pretag }}^{162 \mathrm{pb}^{-1}}$ can be estimated :

$$
N_{\text {sig,pretag }}^{162 \mathrm{pb}^{-1}}=\frac{\left(N_{t a g}-N_{b g}\right)}{\varepsilon_{t a g}}
$$

The estimated number of non-tagged signal events is :

$$
N_{\text {sig,non-tag }}^{162 \mathrm{pb}^{-1}}=\frac{\left(N_{t a g}-N_{b g}\right)}{\varepsilon_{t a g}}-\left(N_{t a g}-N_{b g}\right) .
$$

We additionally analyze $32 \mathrm{pb}^{-1}$ of data taken without the good silicon condition. In this data sample, we do not trust the $b$-tagging information and all the candidate events are categorized in the 0 tag sample. The estimated number of non-tagged (= pretag) signal events in this $32 \mathrm{pb}^{-1}$ is :

$$
N_{\text {sig, }, \text { non-tag }}^{32 \mathrm{pb}^{-1}}=\frac{\left(N_{t a g}-N_{b g}\right)}{\varepsilon_{\text {tag }}} \times \frac{32}{162}
$$

Using the last two equations, we obtain the number of signal events in our 0 tag sample :

$$
N_{\text {sig,non-tag }}=N_{\text {sig,non-tag }}^{162 \mathrm{pb}^{-1}}+N_{\text {sig,non-tag }}^{32 \mathrm{pb}^{-1}}
$$

After applying the efficiency of the $\chi^{2}<9$ cut (91.5\% from Monte Carlo study) and the efficiency of $E_{T}^{4 t h j e t}>21 \mathrm{GeV}$ cut (56\%), we estimate signal events in our 0 tag sample to be $N_{\text {sig,non-tag }}=$ $15.5 \pm 3.2$.

As described in Chapter 11, the number of candidates in the 0 tag sample is found to be 40 . Subtracting $N_{\text {sig,non-tag }}$ calculated above, we estimate the number of background events in this sample to be $24.5 \pm 7.0$.

\section{Summary of the Background Estimation}

We estimated the number of background events for 0,1 and 2 tag samples. For the tag samples, we considered non- $W$, mistag $(W+$ light flavor jets), $W+$ heavy flavor jets, single top and di-boson processes as the possible background source. For each process, we first estimated the estimated number of events with $\geq 1$ SECVTX tagged jets. We obtained the number of background events in our 1 and 2 tag samples by multiplying this number by the efficiencies of the cuts applied after this $b$-tag requirement. In the case of 0 tag sample, we rather estimated the number of signal events in 0 tag sample by dividing the estimated number of signal events in $\geq 1$ SECVTX tag sample by the event tagging efficiency of SECVTX algorithm for $t \bar{t}$ events. We obtained the number of 0 tag background events by subtracting this estimated number of signal from the number of events observed in data.

Summarized in Table 6.7 is the estimated number of background events in our 0,1 and 2 tag samples. As we describe in the next section, we categorize our background sources into four different groups - mistag, $W+$ heavy flavor, $s$ - and $t$ - channels of single top processes for the 1 and 2 tag samples. The table presents the backgrounds due to this categorization. 


\begin{tabular}{|c|c|c|c|}
\hline \hline Mass Template Source & 0 tag & 1 tag & 2 tag \\
\hline mistag & - & $4.3 \pm 0.8$ & $0.066_{-0.008}^{+0.19}$ \\
$W+$ heavy flavor & - & $1.9 \pm 0.6$ & $0.17 \pm 0.05$ \\
single top ( $s$-chan.) & - & $0.2 \pm 0.02$ & $0.037 \pm 0.008$ \\
single top ( $t$-chan.) & - & $0.1 \pm 0.01$ & $0.011 \pm 0.004$ \\
\hline total & $24.5 \pm 7.0$ & $6.5 \pm 1.2$ & $0.28_{-0.05}^{+0.20}$ \\
\hline \hline
\end{tabular}

Table 6.7: Summary of the Background Estimation for the Jet Probability cut of 0.05 .

\subsubsection{Background Template Building}

As we have discussed, our background is composed of several different physics processes, especially in the case of tagged samples. For these samples, we need to produce a reconstructed mass distribution with all the background sources combined. However, we failed to produce reliable distributions for some of the background sources, and decided to use distributions from other processes for these sources.

non- $W$ We can extract a distribution looking at events with non-isolated leptons using data. However, only a few dozen of $\geq 1$ SECVTX tagged events passed our $\chi^{2}$ cut. Within the statistics, it was checked that the distribution thus obtained is consistent with the distribution for mistag [53]. We use the mistag shape to model the non- $W$ background.

$W c \bar{c}, W c$ We use the corresponding ALPGEN Monte Carlo samples for the studies of these processes. The number of Monte Carlo events passing through all our 2 tag sample cuts was only few dozen. The reconstructed mass distributions were shown to be the same as $W b \bar{b}$ within statistics for $\geq 1$ SECVTX tagged events [53]. We represent the shapes of these processes by that of $W b \bar{b}$.

$W W / W Z$ Because of it is expected to make a very small contribution to the total background, we rather chose to simply represent its shape by another process - $W b \bar{b}$.

So, our strategy is only to consider three categories :

(a) $W$ plus heavy flavor can be represented by $W b \bar{b}$ Monte Carlo sample.

(b) mistag and non- $W$ samples are represented by mistag shape.

(c) Single top shapes are taken from corresponding Monte Carlo samples separately.

Considering all the discussions above, we can finally get the template for the background events. We produced the mass distribution shape piling up contributions from the background processes (a) - (c). We used $W b \bar{b}+2$ parton ALPGEN sample for (a), and PYTHIA samples for (c) type backgrounds to describe the reconstructed mass distribution of each process. As for (b) type 
backgrounds, we use the distribution reconstructed by weighting events of $W+$ four light flavor parton ALPGEN + HERWIG Monte Carlo sample by the mistag rate for the event predicted by the mistag matrices. Illustrated in the bottom plots in Figure 6.9 is the reconstruction of the whole template shapes for 1 and 2 tag channels.

On the other hand, the background in 0 tag was not categorized into different physics processes in the estimation of number of background event. The background processes expected to be dominant in $0 \mathrm{tag}$ sample are non- $W$ and $W+$ light flavor jets events. However, modeling of non- $W$ background is only derived using the data, which would end up in a unreliable distribution with small number of entries as in the case of tag samples. Therefore, we simply use $W+$ light flavor jets Monte Carlo sample in the modeling of the 0 tag background shape (top plot in Figure 6.9) ${ }^{4}$.

\section{Background Template Parametrization}

For the background shape, we simply describe it with one function for 1 and 2 tag channels.

$$
f_{b}\left(M_{t}\right)=f_{1}\left(M_{t},\left\{\beta_{1}, \beta_{2}, \beta_{3}\right\}\right)
$$

where $\beta$ 's are the parameters. For the background shape of the 0 tag sample, we add another $f_{1}$ function for a better description of the shape.

$$
f_{b}\left(M_{t}\right)=\beta_{7} \times f_{1}\left(M_{t},\left\{\beta_{1}, \beta_{2}, \beta_{3}\right\}\right)+\left(1-\beta_{7}\right) \times f_{1}\left(M_{t},\left\{\beta_{4}, \beta_{5}, \beta_{6}\right\}\right)
$$

Since the background processes have no dependency on the true mass of the top quark, we only need one template for background, as defined in the previous subsection.

The minimized $\chi^{2}$ for this parametrization for the 2 tag events was

$$
\frac{\chi^{2}}{\text { d.o.f. }}=\frac{28.9}{34}
$$

, while, for the 1 tag was

$$
\frac{\chi^{2}}{\text { d.o.f. }}=\frac{36.7}{32}
$$

and for the 0 sample was

$$
\frac{\chi^{2}}{\text { d.o.f. }}=\frac{28.9}{34}
$$

\footnotetext{
${ }^{4}$ One might suspect the "real" distribution of the background residing in our data samples could be different from the template shape obtained in such a Monte Carlo driven way. A study was performed to investigate into this issue in the Run I period of the CDF experiment [64]. They compared the $W+4 j e t s$ template obtained from Monte Carlo sample generated by VECBOS, another Monte Carlo generator with the leading order calculation, to the distribution of $Z+4 j$ ets events in data. The properties of $Z+$ jets events is similar to $W+$ jets events in hadron collider, except for the decay modes of $Z$ and $W$ bosons. They found seven $Z+4 j e t s$ candidate events with the $Z$ decaying leptonically in the Run I data. The calculation the reconstructed top mass for $Z+j e t s$ events was done by assigning one of the two leptons in the $Z$ decay to the neutrino in the $\chi^{2}$ fitter. Both combination in terms of the lepton-to-neutrino assignment was tried. The distribution of $14 Z+4$ jets candidates was found to be consistent with the Monte Carlo template within the statistics.
} 


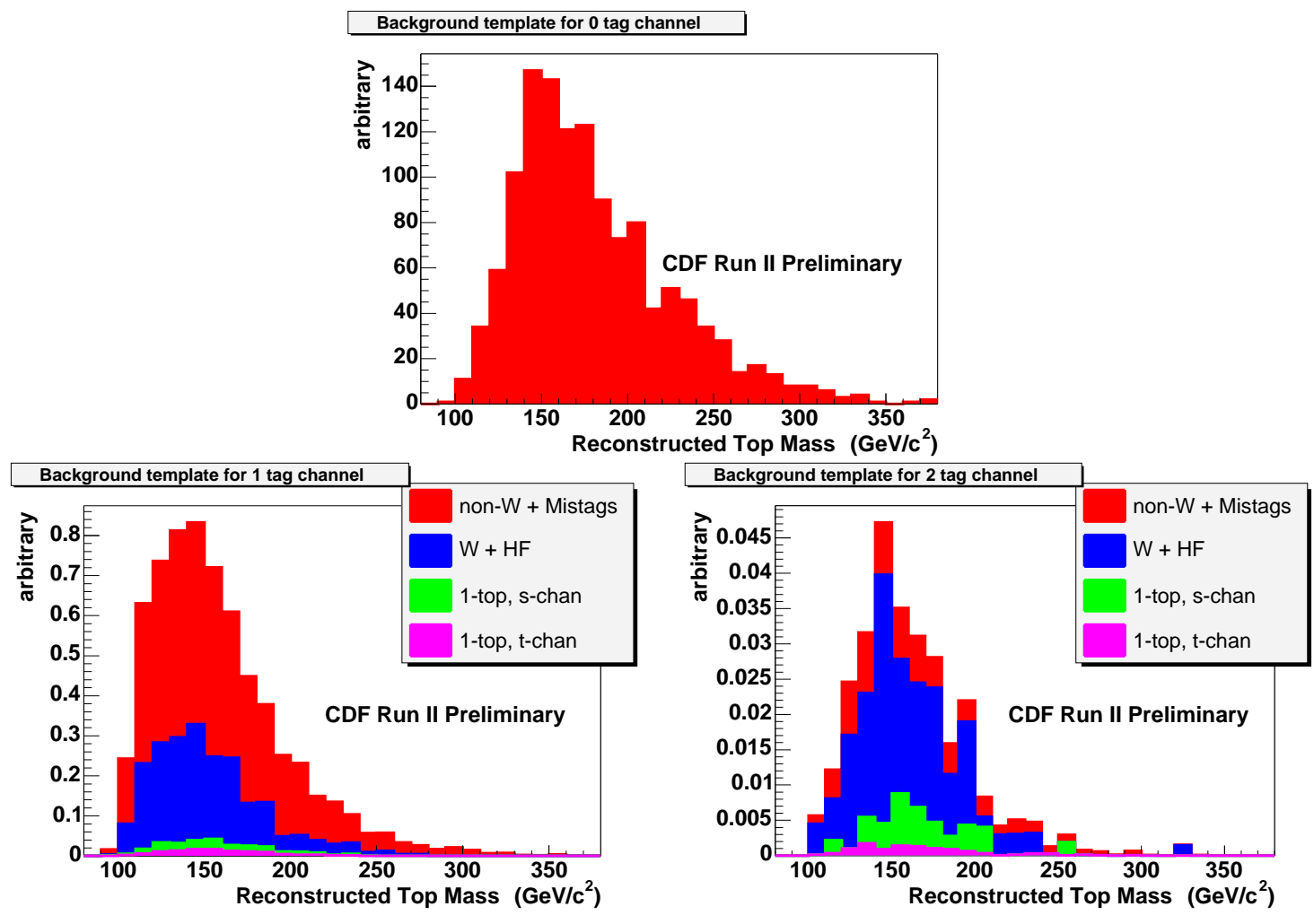

Figure 6.9: Background templates for 0 tag (top) 1 tag (bottom left) and 2 tag (bottom right) samples. Contribution from each different process is shown in different color for 1 and 2 tag samples.

We summarize the $\beta$ parameters of the 0,1 and 2 tag samples in Table 6.8. The background template along with its fitted curve is shown in Figure 6.10.

\subsection{Summary of the Templates}

As a summary of the template fittings, we plot the obtained shape functions in Figure 6.11. This plot illustrates how the reconstructed mass distribution shifts its shape as a function of the true top mass, as well as a comparison with the background shape. 


\begin{tabular}{|c|ccc|ccc|ccc|}
\hline \hline$i$ & \multicolumn{3}{|c|}{$\beta_{i}(0 \mathrm{tag})$} & \multicolumn{3}{c|}{$\beta_{i}(1 \mathrm{tag})$} & \multicolumn{3}{c|}{$\beta_{i}(2 \mathrm{tag})$} \\
\hline 1 & 91.0 & \pm & 3.8 & 94.0 & \pm & 0.6 & 98.4 & \pm & 6.4 \\
2 & 2.78 & \pm & 0.44 & 1.87 & \pm & 0.10 & 2.43 & \pm & 0.97 \\
3 & 0.0403 & \pm & 0.0035 & 0.0447 & \pm & 0.0015 & 0.0539 & \pm & 0.0111 \\
4 & 130.5 & \pm & 4.9 & & - & & & - & \\
5 & 1.66 & \pm & 1.31 & \multicolumn{3}{|c}{-} & & \multicolumn{3}{|c}{-} \\
6 & 0.1105 & \pm & 0.0572 & \multicolumn{3}{|c}{-} & & \multicolumn{3}{|c}{-} \\
7 & 0.8697 & \pm & 0.0475 & \multicolumn{3}{|c}{-} & & \multicolumn{3}{|c}{-} \\
\hline \hline
\end{tabular}

Table 6.8: $\quad \beta$ parameters obtained in the background template fitting for the zero, single and double tag samples.

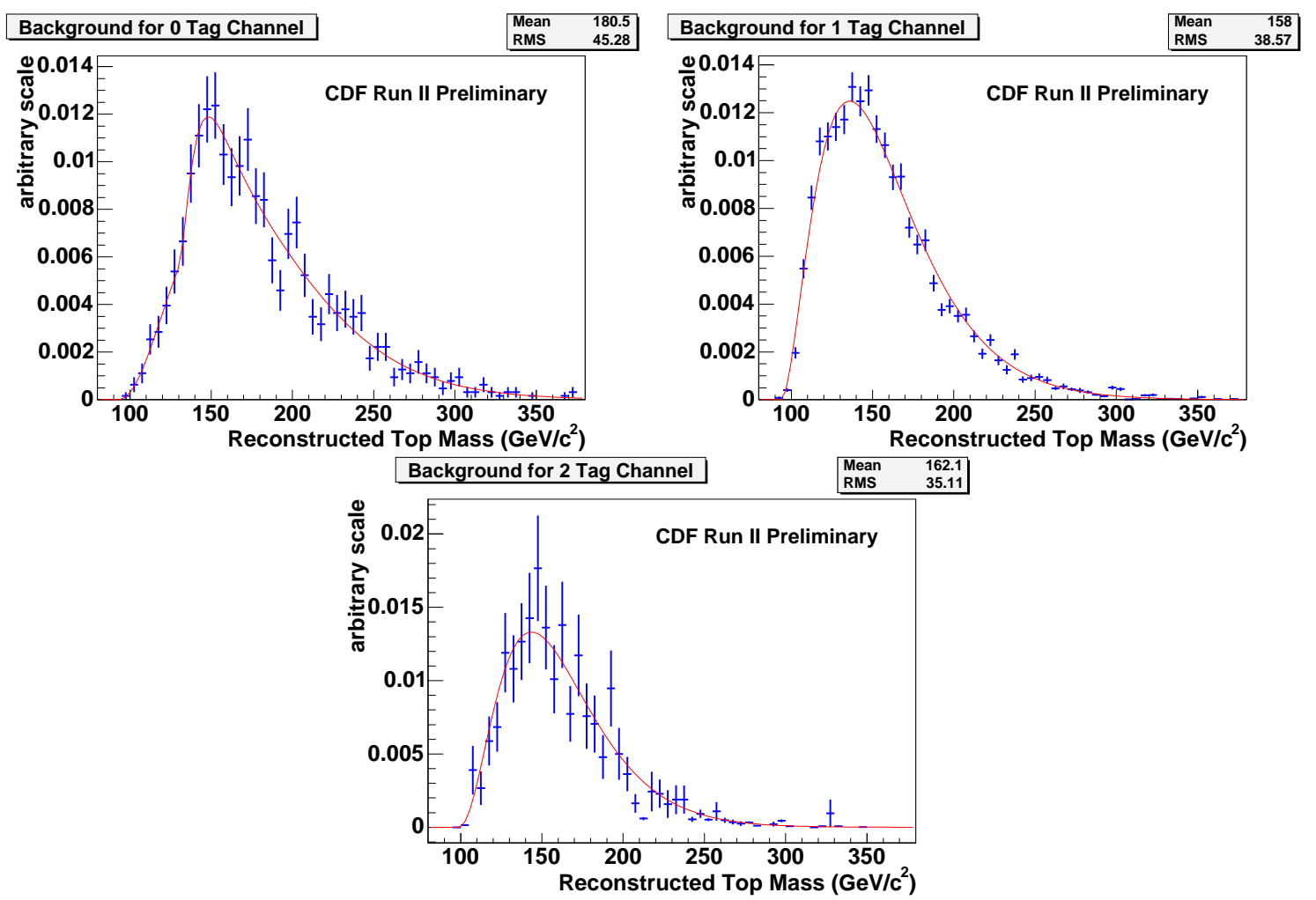

Figure 6.10: Background template along with the background shape function. Left-top: the background template for the no-tag, right-top: for single tag, bottom: for the double tag. 

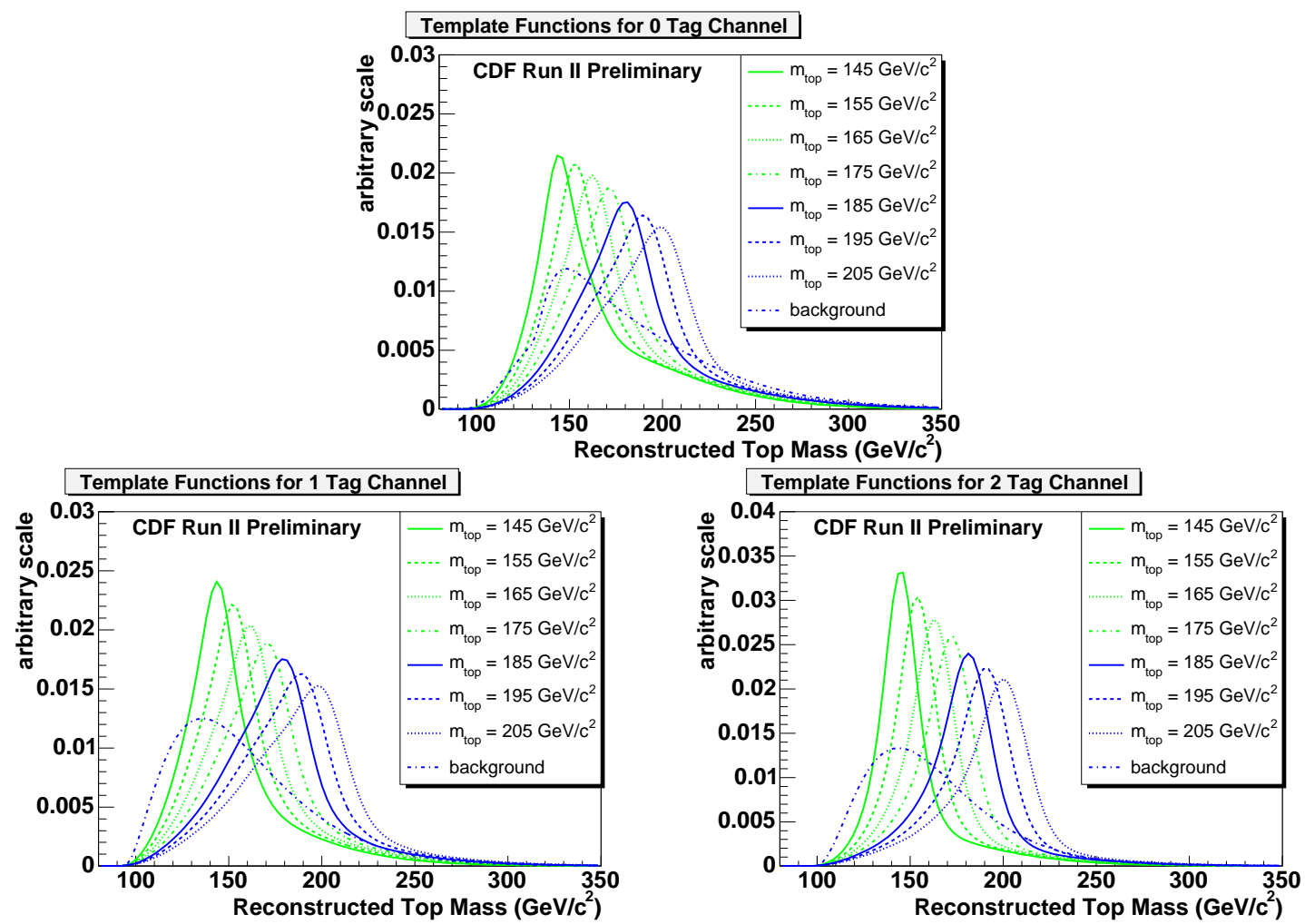

Figure 6.11: The obtained functions for signal events with various true top masses. The parametrized background shape is also shown. Functions are normalized to the equal area. Top figure shows 0 tag case, Left figure shows 1 tag case, while right figure shows the 2 tag case. 


\section{Chapter 7}

\section{Determination of the Top Quark Mass}

In this chapter, we illustrate the method with which we extract the top quark mass using the selected event sample. We also discuss the way to perform the pseudo-experiments, which will be used in the validation of the measurement scheme, the estimation of the expected statistical sensitivity of our analysis on the top quark mass and the estimation of the systematic uncertainty.

\subsection{Determination of the Top Quark Mass}

We measure the top quark mass by fitting the reconstructed top mass distribution in the data sample to a sum of templates for $t \bar{t}$ signal and background using an unbinned likelihood method. The likelihood $L$ for each of zero, one or two tag channel is composed as follows :

$$
L=L_{\text {shape }} \times L_{b k g}
$$

where $L_{\text {shape }}, L_{b k g}$ describe the likelihoods for the top mass distribution, and the number of background events.

The definitions of these likelihoods are :

$$
\begin{aligned}
L_{\text {shape }} & =\frac{e^{-\left(N_{s}+N_{b}\right)}\left(N_{s}+N_{b}\right)^{N}}{N !} \Pi_{i=1}^{N \text { events }} \frac{N_{s} f_{s}\left(M_{t}^{i} \mid m_{t}, \vec{\alpha}\right)+N_{b} f_{b}\left(M_{t}^{i} \mid \vec{\beta}\right)}{N_{s}+N_{b}} \\
L_{b k g} & =\exp \left(-\frac{1}{2}\left[\frac{N_{b}-N_{b}^{\text {pred }}}{\sigma_{N_{b}^{\text {pred. }}}^{2}}\right]^{2}\right)
\end{aligned}
$$

$M_{t}^{i}$ is the reconstructed top mass for each event in data sample. In this fit, $m_{t}, N_{s}$ and $N_{b}$ are the free parameters describing the true top mass, number of signal and background events, respectively. $N$ is the total number of events in the sample to be fitted and $\vec{\alpha}$ and $\vec{\beta}$ are the 
template parameters obtained in Section $6 . N_{b}^{\text {pred. }}$ and $\sigma_{N_{b}^{\text {pred. }}}$. refer to the number of background events and its error estimated in the previous chapter.

$L_{\text {shape }}$ is composed of the probability of the $M_{t}^{i}$ in the supposed template shape. This term extracts the template distribution which reproduces the $M_{t}^{i}$ distribution in data sample by adjusting the $m_{t}$ and background fraction in the likelihood fit. On the other hand, $L_{b k g}$ constrains the number of background events around the estimated number.

The actual fitting is performed using the MINUIT package, where the minimum for the log likelihood $-\log L$ is searched for. The upper and lower statistical errors on the measured top quark mass is calculated by searching for the top quark mass with which $-\log L$ becomes larger than the minimum by 0.5 .

In order to combine the results from 0,1 and the 2 tag samples, we multiply the three likelihoods :

$$
L^{\text {combine }}=L^{0 t a g} \times L^{1 t a g} \times L^{2 t a g}
$$

These samples are statistically independent, so the extension of the likelihood is smooth. The top quark mass $m_{t}$ is the common for three likelihoods, while the number of signal and background $N_{s}$ and $N_{b}$ are varied independently for the three samples in the fit.

\subsection{Method of the Pseudo-experiments}

We perform pseudo-experiments in order to validate our measurement method, estimate the expected statistical sensitivity of our method on top quark mass and to estimate the systematic uncertainty in the following chapters. We describe our method of the pseudo-experiments in this section.

In a pseudo-experiment, we make a set of reconstructed top masses (called pseudo-sample), and perform the likelihood fit supposing this pseudo-sample were a sample extracted from data. The best way to make such a pseudo-sample would be to use Monte Carlo events passing through all the cuts for the event sample category. However, we have to perform the pseudo-experiment many times in order to obtain reliable estimates for the performance of our method. This would require a huge Monte Carlo samples for signal and all the background processes, which consumes a large time scale for the generation of the Monte Carlo samples. We rather choose an alternative method, in which we generate random numbers due to the template histograms obtained in Monte Carlo studies. Each random number thus generated is assumed to represent the reconstructed top mass of an event. Desired numbers of reconstructed masses are generated for signal and background processes, and are used as the pseudo-sample in our pseudo-experiments.

The numbers of signal and background events input to the pseudo-experiments slightly affect the results. In order to obtain reliable results from the pseudo-experiments, we need to input proper numbers of signal and background events to the pseudo-experiments. In our pseudoexperiments, we usually determine the number of signal events using the efficiency estimated 
in Table 6.4. By multiplying the integrated luminosity and the theoretical $t \bar{t}$ production cross section of $6.7{ }_{-0.9}^{+0.7} \mathrm{pb}$ to the efficiency, we can obtain the center value for the number of signal events ${ }^{1}$. The number of signal events input to each pseudo-experiments is defined by generating a random number due to the Poisson distribution with the mean value set to the center value. As for the number of background events, we first determine the center value by generating a random number due to Gaussian distribution with mean and sigma set to the center value and uncertainty in our background estimate ("total" in Table 6.7). This center value is defined in each pseudoexperiment. The number of background input to the pseudo-experiment is determined by the Poisson statistics with the mean value set to this center value. This method of determining the numbers of signal and background events is used in estimating the performance of our likelihood fitter. One of such cases is the sensitivity study (described in Chapter 8).

We use an alternative method of determination of the numbers of signal and background events when we desire to reproduce the situation of the data analysis as well as possible in the pseudo-experiments. The sanity check study (described in Chapter 9) and the estimation of the systematic error (in Chapter 10) are such cases. In this method, the number of background events is determined in the same way as described above. However, we define the number of signal events by subtracting the number of background from the total number of events in order to keep the total number of events in each pseudo-experiment the same as that in the data analysis.

\footnotetext{
${ }^{1}$ Although the numbers in the table are obtained in Monte Carlo studies and must be multiplied by the datato-Monte Carlo scale factor for lepton identification efficiency and the trigger efficiency measured using data, the effect in total is only within $10 \%$. The typical Poisson fluctuation is greater than this, so we simply neglect these factors and use the numbers in the table.
} 


\section{Chapter 8}

\section{Sensitivity Study}

In this chapter, we present the results of our sensitivity study. As we discussed in Section 3.3, the cut on Jet Probability is tunable, although the use of this algorithm requires a calibration study offering the data-to-Monte Carlo scale factor for the heavy flavor tagging efficiency and the mistag matrix specific for the cut value. We perform pseudo-experiments and tune the cut on Jet Probability so that the expected statistical error on the measured top quark mass becomes the minimum in Section 8.1. We recycle the double tag events failing the event selection cuts of 2 tag sample into the 1 tag event selection, as described in Section 4.4. We perform pseudoexperiments in order to find out whether the recycling works to further improve the sensitivity of our method to the mass of the top quark in Section 8.2.

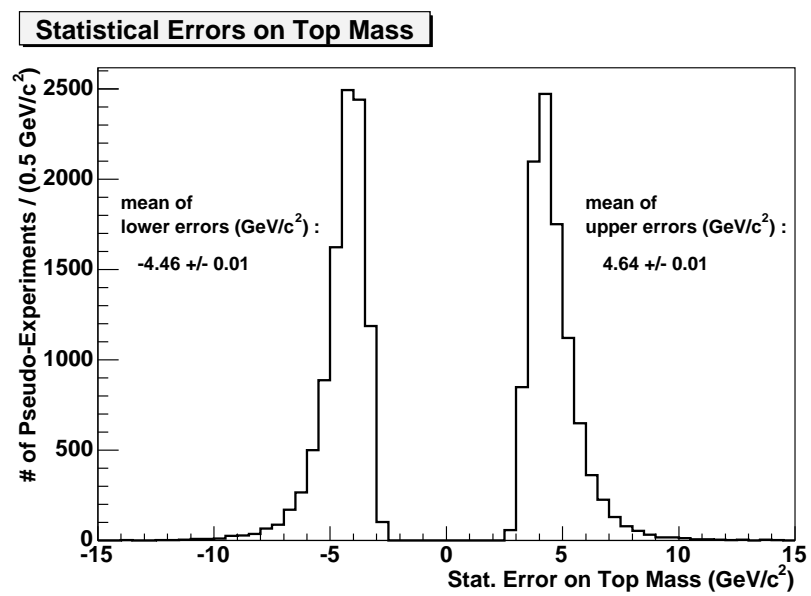

Figure 8.1: Upper and lower statistical error on measured top quark mass in pseudo-experiments. Mean values for upper and lower error are also shown.

Figure 8.1 shows the upper and lower statistical errors on top quark mass measured in the combined fit of 0,1 and 2 tag samples in 10000 pseudo-experiments with the input top quark 
mass of $175 \mathrm{GeV} / c^{2}$. This set of pseudo-experiments assumed the integrated luminosity of $162 \mathrm{pb}^{-1}$ and the theoretical $t \bar{t}$ cross section of $6.7_{-0.9}^{+0.7} \mathrm{pb}$. The cut on Jet Probability was 0.05 , and the recycling of the 2 tag events was enabled in this set of pseudo-experiments. The means of upper and lower errors are also shown in the figure. We perform pseudo-experiments with different Jet Probability cut or recycling settings and calculate the means of the errors, which are taken as the expected statistical errors of our method through this chapter.

\subsection{Optimization of the Jet Probability Cut}

We perform pseudo-experiments with different cut values for Jet Probability tagging in this section in order to find out the optimum cut that gives the best sensitivity to the mass of the top quark. We perform the pseudo-experiments for 1 and 2 tag samples along with the combined fits of these two samples. The pseudo-experiments are performed assuming $200 \mathrm{pb}^{-1}$ for the integrated luminosity. The sensitivity of the 0 tag channel is independently estimated and will be combined to the combined sensitivity of 1 and 2 tag samples in Subsection 8.1.4.

\subsubsection{Expected Number of Events for 1 and 2 Tag Samples}

As mentioned in Section 5.3, our 2 tag sample is a part of the inclusive SECVTX tagged sample ( $\geq 1$ SECVTX tagged sample). It is worth while commenting two details. One is how to deal with the event which fails $\chi^{2}$ or $m_{j j}$ cut in 2 tag sample : such an event is fed back to the 1 tag sample selection criteria, where the $b$-tagging information of Jet Probability is neglected and the event has a chance to pass the $\chi^{2}$ cut even though it failed the $\chi^{2}$ cut in the 2 tag sample selection. We will present our results concerning this recycling in Section 8.2. The other is how to deal with the events which have three or more $b$-tagged jets : Such an event is forced down to a double tag candidate by neglecting the third and fourth tagged jets. In such a case, SECVTX tagged jets have the first priority. Jets which have lower Jet Probability value has the next priority. So, for example, if we have two SECVTX tagged jets and one JP tagged jet, the JP tagged jet is discarded. This method of sub-division of the tagged samples conserves the total number of events in 1 and 2 tag samples, regardless of our cut value on Jet Probability.

Assuming $200 \mathrm{pb}^{-1}$ for the integrated luminosity and $6.7 \mathrm{pb}$ for the $t \bar{t}$ pair production cross section, we have the expected number of signal events shown in Table 8.1. For the cut values other than 0.01 and 0.05 , the Jet Probability algorithm has not been calibrated [44, 45]. So we extrapolated the data-to-Monte Carlo scale factors as follows :

$$
S F_{J P c u t}=\frac{\left(S F_{0.05}-S F_{0.01}\right)}{(0.05-0.01)} \times(J P c u t-0.01)+S F_{0.01}
$$

where $S F_{0.01}$ and $S F_{0.05}$ are the measured values of the scale factors for the calibrated points, and $S F_{J P c u t}$ is the scale factor extrapolated for cut value of JPcut. By assuming the scale factor in this way, we can measure the efficiency of our event selection for each sample at an arbitrary 


\begin{tabular}{|c|c|c|}
\hline \hline JPcut & \# of $1 \mathrm{tag}$ & \# of $2 \mathrm{tag}$ \\
\hline none & 35 & 7 \\
0.01 & 31 & 11 \\
0.05 & 28 & 14 \\
\hline \hline
\end{tabular}

Table 8.1: $\quad$ Expected number of $t \bar{t}$ signal events for different Jet Probability cuts assuming $200 \mathrm{pb}^{-1}$ for the integrated luminosity and $6.7 \mathrm{pb}$ for the theoretical cross section. "None" means we only use SECVTX tagging for the selection of $2 \mathrm{tag}$ sample. These two Jet Probability cut values are those for which the algorithm is calibrated.

cut on Jet Probability, and can calculate the expected number of signal events. The estimation of the number of background events is performed also in a very ad-hoc way. The background estimation is only possible for Jet Probability cuts of $0.00,0.01$ and 0.05 in the same way as in Chapter 6 , where the cut of 0.00 corresponds to the case where we only use SECVTX tagging in the double tagging of events. We fit the estimation obtained at these three points into a linear function

$$
B_{k g d}{ }_{J P u t}=a \cdot J P c u t+b
$$

where $B k g d_{J P c u t}$ and JPcut each refers to the expected number of background events and the cut on Jet Probability, $a$ and $b$ are the parameters determined in the fitting. By using the values of $a$ and $b$ obtained in the fit, we estimated the number of background for arbitrary cut value on Jet Probability. The expected number of signal and background events in this way are plotted for several points in the Jet Probability cut range of 0.001 to 0.7 in Figure 8.2. The point at 0.0001 shows the number of events for the case where Jet Probability is not used for the double tagging. Note that the total number of events stays constant through the scanned cut value region.

\subsubsection{Templates for the Mass Fitting at Various Jet Probability Cuts}

Looser tagging of the second $b$-jet provides increase of the signal events in the 2 tag sample, but might provide more combinatorial background to 2 sample since a charm quark coming from $W$ boson decay may be tagged as the second $b$-jet, though the branching ratio of $W \rightarrow c s$ is the half of the hadronic $W$ decay and the Jet Probability tagging efficiency for charm jet is supposed to be smaller than for $b$-jets. Figure 8.3 shows the signal templates of 2 tag sample for different cut values on Jet Probability. We can, in fact, see that the fraction of the wrongly reconstructed events in the templates increases as the cut value gets larger. We show the signal templates of 1 tag sample for different cut values on Jet Probability in Figure 8.4. The fraction of correct combination decreases with larger cut value on Jet Probability also for 1 tag sample. This is due to the fact that the fraction of recycled events, where the fraction correct combination is presumably small because these events are already rejected at the 2 tag sample, in the 1 tag 
Number of Events

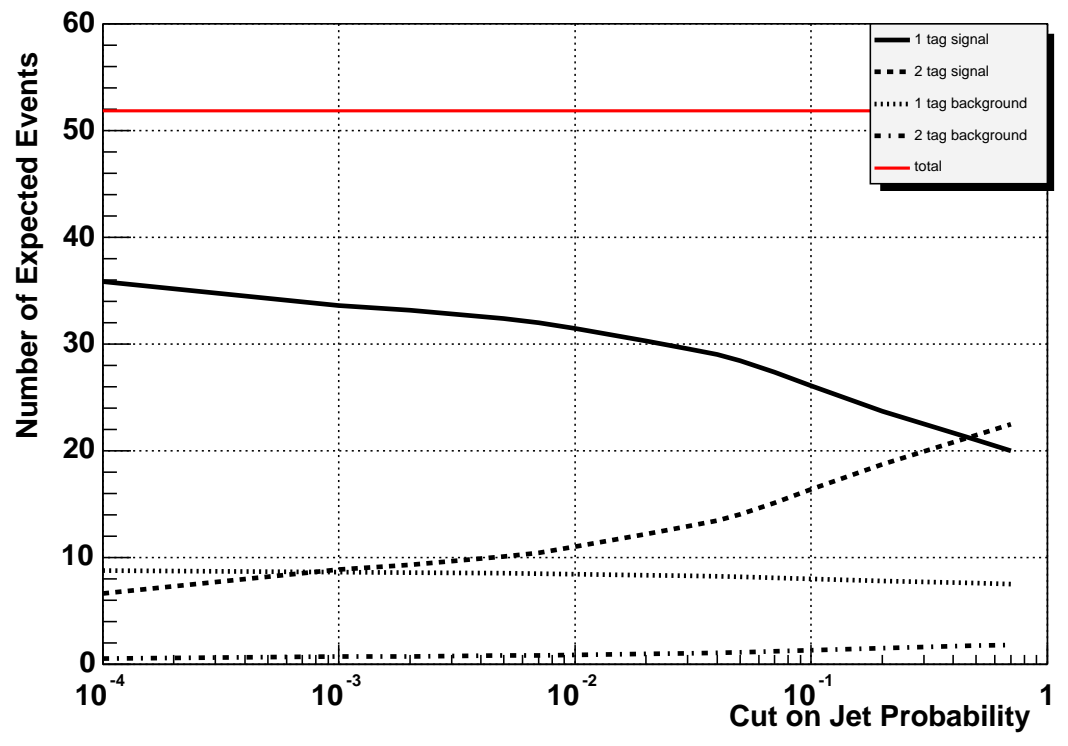

Figure 8.2: Number of signal and background events as a function of the Jet Probability cut.

sample increases.

For the signal template parametrization, we made histograms for 12 different top mass inputs (from 150 to $205 \mathrm{GeV} / c^{2}$ with $5 \mathrm{GeV} / c^{2}$ step) and fit them simultaneously, in the method described in Chapter 6. Due to the above discussion, the signal template parametrization is affected by the increase in the fraction of combinatorial background. Figures 8.5 and 8.6 show the 18 parameters for the signal template functions at various JP cut thresholds from 0.001 to 0.2 for 1 and 2 tag samples. The most left point $\left(\mathrm{JPcut}=10^{-4}\right.$ ) shows the SECVTX only case. The definition of the parameters, $\alpha \mathrm{s}$, are the same in the subsection 6.1 .

Parameter $\alpha 8$ shows the fraction of the $f_{1}$ function, which presumably corresponds to the fraction of combinatorial background. Behavior of $\alpha 8$ of 1 tag sample looks as expected since the fraction of combinatorial background increases as the recycled events with two tagged jets increases with looser double tagging condition. For the 2 tag case, it is expected that $\alpha 8$ becomes larger as the cut on Jet Probability is relaxed due to increased charm tagging. However, the behavior below 0.01 is not consistent with this assumption. This may be due to the MC statistics, but may also be due to methodology itself. This template variation affect the sensitivity in addition to the low statistics of each pseudo-experiment.

As for the background template in the scanning of the cut on Jet Probability, we simply used $W b \bar{b}+2$ parton ALPGEN Monte Carlo sample. 

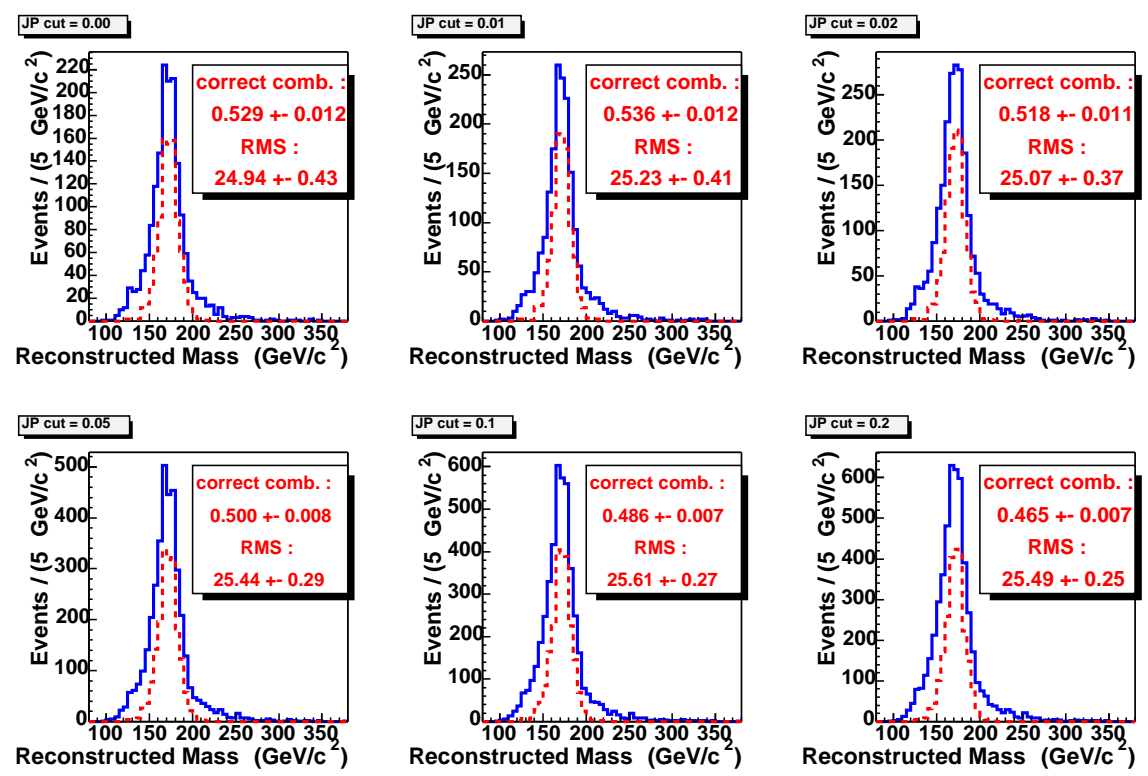

Figure 8.3: 2 tag signal templates for different cut values on Jet Probability. The cut values are from left on top column : 0.00 (the case where Jet Probability is not used), 0.01 and 0.02 from left on bottom column : $0.05,0.1$ and 0.2 . In the top-right box in each plot are the fraction of correct combination, along with the RMS of the solid histogram.

\subsubsection{Statistical Sensitivity with 1 and 2 tag samples}

Using the templates described in the previous section, we performed 5000 pseudo-experiments for each Jet Probability cut. Figure 8.7 shows the results of this sensitivity study for 1 tag, 2 tag and these two samples combined. The expected lower and upper statistical errors are plotted as a function of the cut value on Jet Probability. Figure 8.8 shows the same plot, but zooming into the region of combined results. The point at the Jet Probability threshold of $10^{-4}$ shows results using only SECVTX. Also shown is the $\geq 1 b$-tag case (inclusive SECVTX sample measurement), where all the events having $\geq 1$ SECVTX tagged jets are used as one sample for the top mass measurement.

Table 8.2 summarizes the quantitative numbers from some representative points. Compared to the inclusive SECVTX measurement, we have $5 \sim 6 \%$ improvement at the best point. Even with the current calibrated point (i.e. JPcut $=0.05$ ), we still have $4 \sim 5 \%$ improvement over the inclusive SECVTX measurement. 

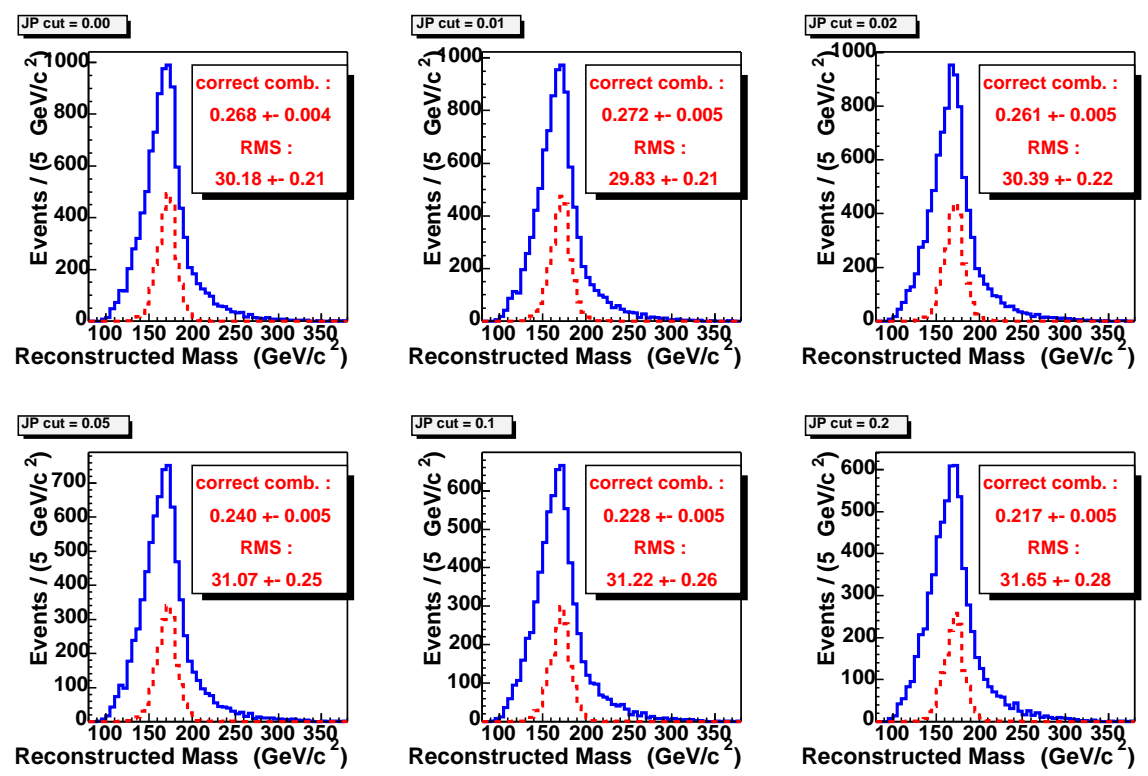

Figure 8.4: 1 tag signal templates for different cut values on Jet Probability. The cut values are from left on top column : 0.00 (the case where Jet Probability is not used), 0.01 and 0.02 from left on bottom column : $0.05,0.1$ and 0.2 . In the top-right box in each plot are the fraction of correct combination, along with the RMS of the solid histogram.

\subsubsection{Adding 0 tag sample}

The change in cut threshold on Jet Probability does not affect 0 tag sample. This enables us to add the 0 tag sample to the combination by the following formula :

$$
\frac{1}{\sigma_{\text {combined }}^{2}}=\frac{1}{\sigma_{1,2 \text { tag }}^{2}}+\frac{1}{\sigma_{0 t a g}^{2}}
$$

where $\sigma_{\text {combined }}$ is the statistical sensitivity with the three channels combined, $\sigma_{1,2 t a g}$ and $\sigma_{0 t a g}$ are the statistical sensitivity of 1 and 2 tag samples combined, and of 0 tag sample, respectively. From a set of pseudo-experiments, we obtained $\sigma_{0 t a g}=10.3 \pm 0.2 \mathrm{GeV} / c^{2}$ for $200 \mathrm{pb}^{-1}$. Figure 8.9 shows the expected statistical sensitivity of the top mass measurement with 0,1 and 2 tag samples combined. The point at the Jet Probability threshold of $10^{-4}$ shows results using only SECVTX. Also shown is the $\geq 1 b$-tag case (inclusive SECVTX sample measurement), where all the events having $\geq 1$ SECVTX tagged jets are used as one sample, combined with 0 tag sample.

Table 8.3 summarizes the expected statistical sensitivity for several representative cut values on Jet Probability. The improvement over the case where $b$-tagged events are not subdivided is also summarized in percentage (\%). Even after including 0 tag sample in the combined measurement, the improvement is expected to be $\sim 4.5 \%$ at the cut of 0.2 , and $\sim 4.0 \%$ for the calibrated point (cut value 0.05). 


\section{One tag template parameters at various JP cuts}
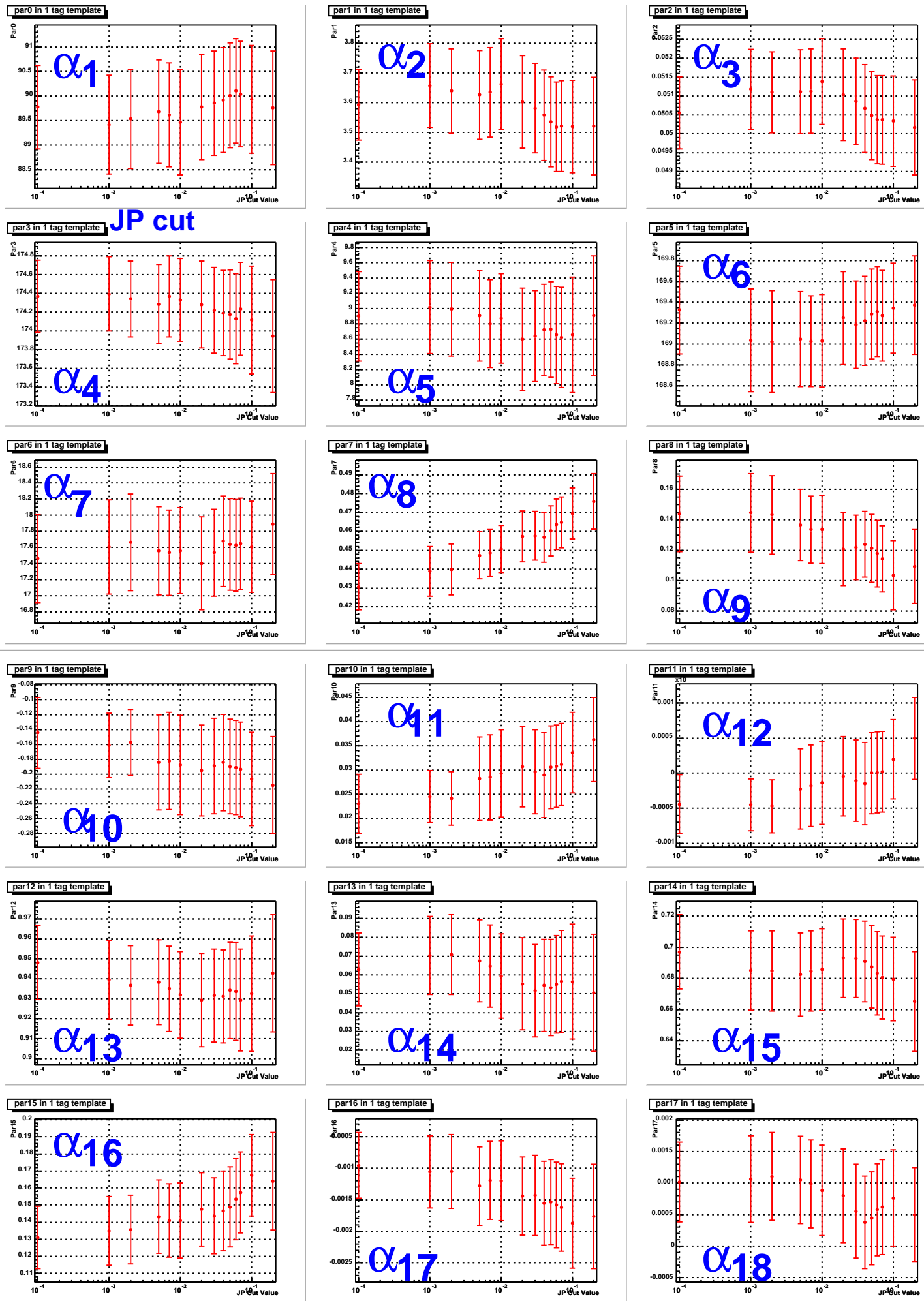

Figure 8.5: Eighteen parameters for the top mass template at the various cut values on Jet Probability for 1 tag sample. 


\section{Two tag template parameters at various JP cuts}
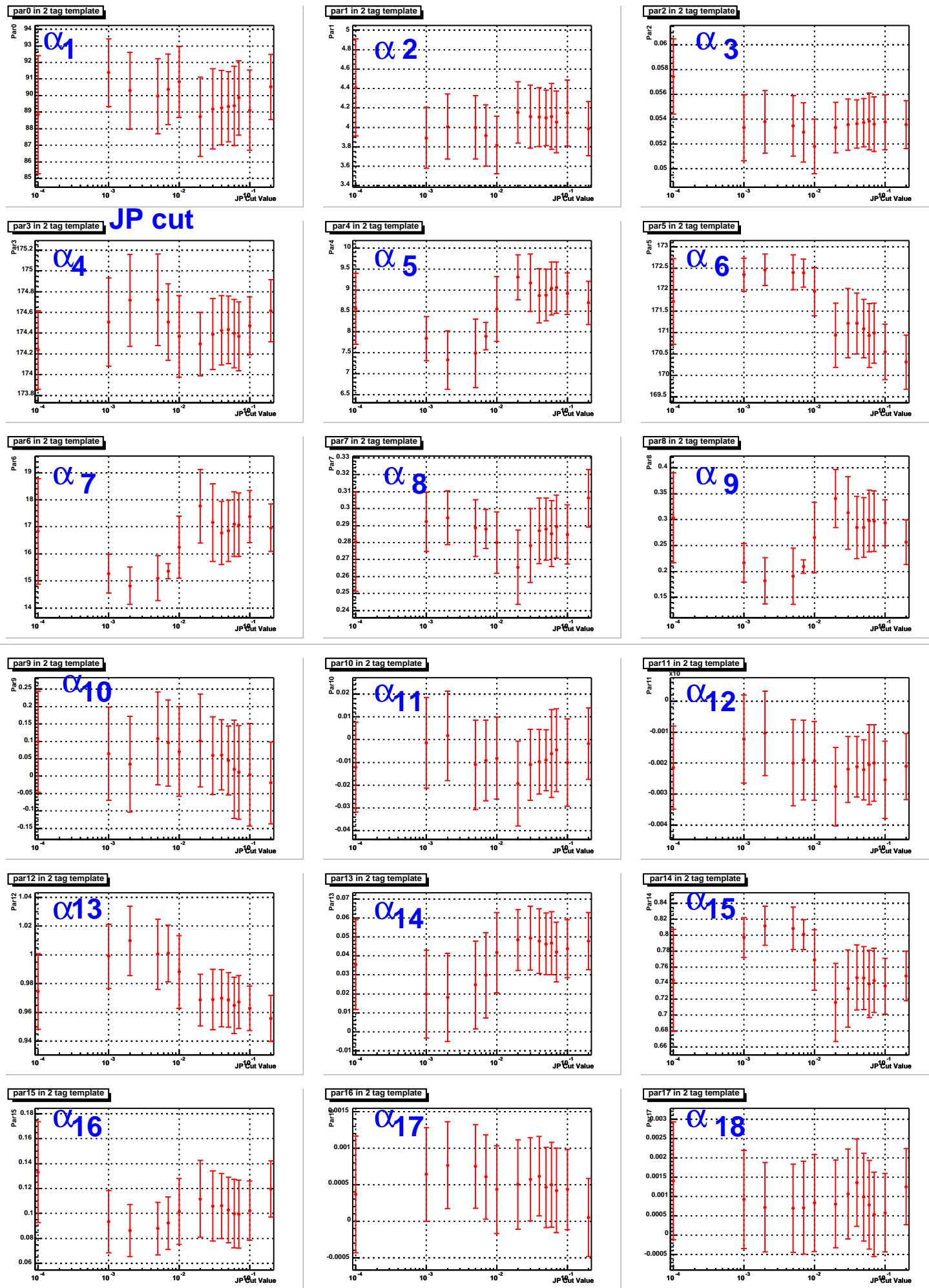

Figure 8.6: Eighteen parameters for the top mass template at the various cut values on Jet Probability for 2 tag sample. 

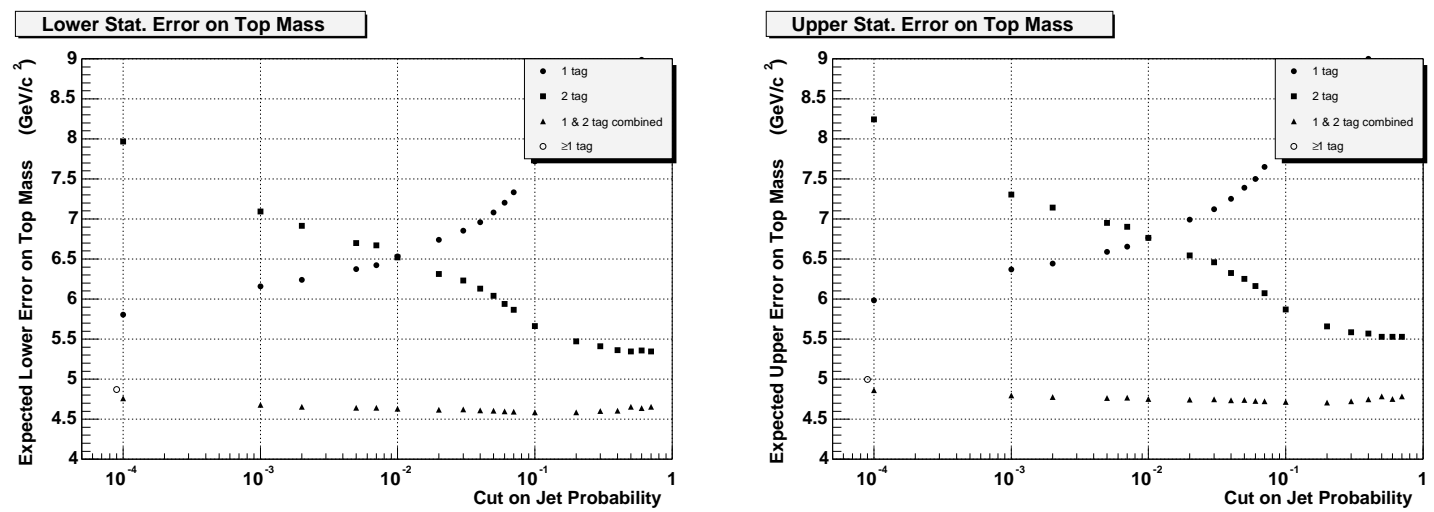

Figure 8.7: Statistical sensitivity of 1 and 2 tag samples for integrated luminosity of $200 \mathrm{pb}^{-1}$ as a function of the Jet Probability cut. Left: expected lower statistical error. Right: expected upper statistical error. JPcut $=10^{-4}$ points show the case where only SECVTX tagger is used, and open circle corresponds to $\geq 1$ tag case.
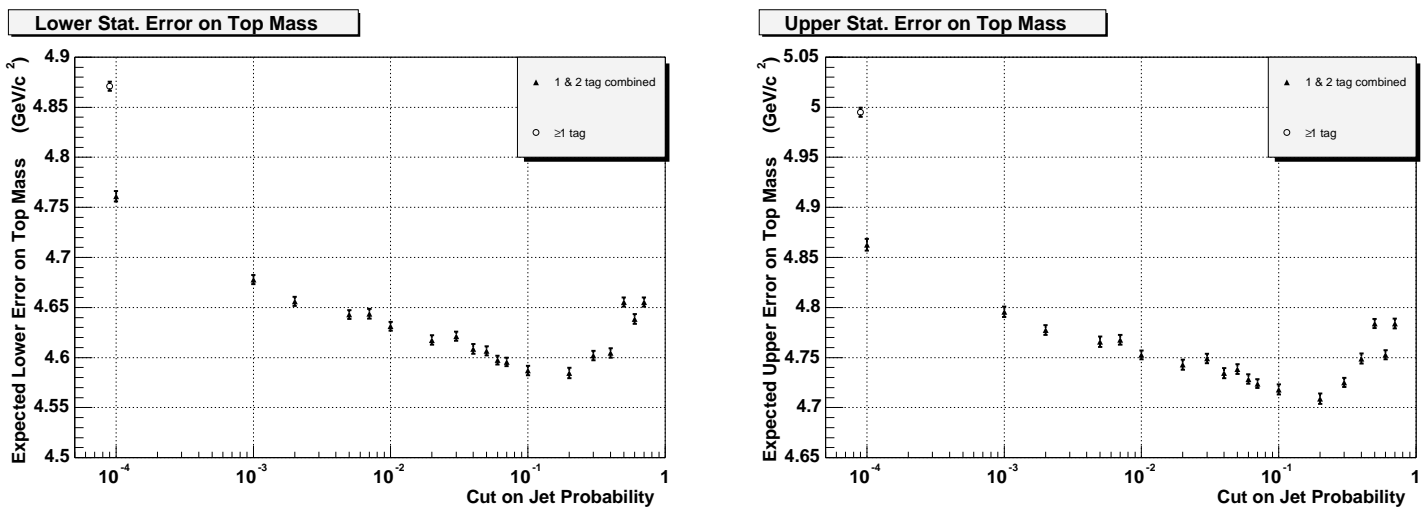

Figure 8.8: Statistical sensitivity of combined fit of 1 and 2 tag samples for integrated luminosity of $200 \mathrm{pb}^{-1}$ as a function of the Jet Probability cut. Left: expected lower statistical error Right: expected upper statistical error. JPcut $=10^{-4}$ point shows the case where only SECVTX tagger is used, and open circle corresponds to $\geq 1$ tag case.

\begin{tabular}{|c|c|c|c|c|}
\hline \hline JP cut & upper & lower & improve (upper) & improve (lower) \\
\hline none (1 $\geq$ tag) & $4.960 \pm 0.097$ & $4.856 \pm 0.095$ & - & - \\
none (1+2 SECVTX) & $4.863 \pm 0.006$ & $4.761 \pm 0.005$ & $2.0 \%$ & $2.0 \%$ \\
0.01 & $4.753 \pm 0.004$ & $4.631 \pm 0.005$ & $4.1 \%$ & $4.6 \%$ \\
0.05 & $4.739 \pm 0.005$ & $4.607 \pm 0.005$ & $4.4 \%$ & $5.1 \%$ \\
0.5 & $4.695 \pm 0.005$ & $4.573 \pm 0.005$ & $5.3 \%$ & $5.8 \%$ \\
\hline \hline
\end{tabular}

Table 8.2: Statistical sensitivity on the mass of top quark assuming integrated luminosity of $200 \mathrm{pb}^{-1}$ for several representative cuts on Jet Probability for measurement combining 1 and 2 tag samples. 

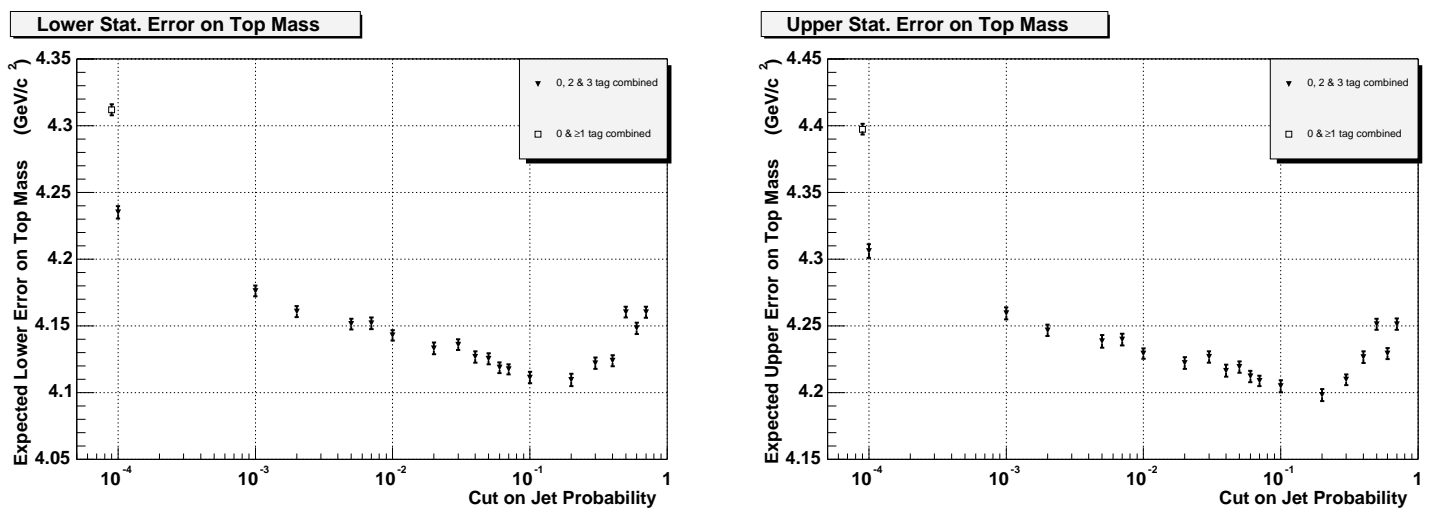

Figure 8.9: Expected statistical sensitivity of the top mass measurement combining 0,1 and $2 \mathrm{tag}$ samples for integrated luminosity of $200 \mathrm{pb}^{-1}$ as a function of the cut threshold on Jet Probability. Left: lower error. Right: upper error. JPcut $=10^{-4}$ point shows the combined results using only SECVTX, and the open circle corresponds to $\geq 1$ tag case (inclusive SECVTX sample measurement).

\begin{tabular}{|c|c|c|c|c|}
\hline \hline JP cut & upper & lower & improve (upper) & improve (lower) \\
\hline none (1 $\geq$ tag) & $4.397 \pm 0.004$ & $4.312 \pm 0.004$ & - & - \\
none (1+2 SECVTX) & $4.306 \pm 0.005$ & $4.235 \pm 0.005$ & $2.0 \%$ & $1.8 \%$ \\
0.01 & $4.229 \pm 0.004$ & $4.143 \pm 0.004$ & $3.8 \%$ & $3.9 \%$ \\
0.05 & $4.219 \pm 0.004$ & $4.125 \pm 0.004$ & $4.0 \%$ & $4.3 \%$ \\
0.2 & $4.198 \pm 0.005$ & $4.109 \pm 0.005$ & $4.5 \%$ & $4.7 \%$ \\
\hline \hline
\end{tabular}

Table 8.3: Expected statistical sensitivity on the mass of top quark with several representative cuts on Jet Probability for integrated luminosity of $200 \mathrm{pb}^{-1}$ in the measurement combining 0 , 1 and 2 tag samples.

\subsubsection{Summary of Sensitivity Study}

We performed a series of pseudo-experiments in order to optimize the cut threshold of the Jet Probability algorithm for the best statistical sensitivity on the measurement of the mass of the top quark in the combined fit to the 0,1 and 2 tag samples. The best optimized btagging condition using Jet Probability is expected to improve the statistical error by $\sim 4.5 \%$ compared to the case where we use the events with SECVTX tagged jets inclusively as one sample and combine it with 0 tag sample. However, Jet Probability has not been calibrated for the corresponding cut threshold (0.2).

At present, Jet Probability cut at 0.05 provides the best result among the calibrating points (i.e. mistag matrix and scale factor are both available), and we use this point for our analysis. At this point, we still estimate an improvement of $\sim 4.0 \%$ on the statistical error on the top quark mass measurement. 


\subsection{Effect of the Event Recycling}

We performed another series of pseudo-experiments in order to understand the effect of recycling the double tagged events that fail 2 tag event selection criteria into the 1 tag sample selection. We performed 10000 pseudo-experiments with and without the recycling of the events, assuming the integrated luminosity of $162 \mathrm{pb}^{-1}$. Figure 8.10 shows the distributions of the statistical uncertainty on the top quark mass with 0,1 and 2 tag samples combined for each case. We

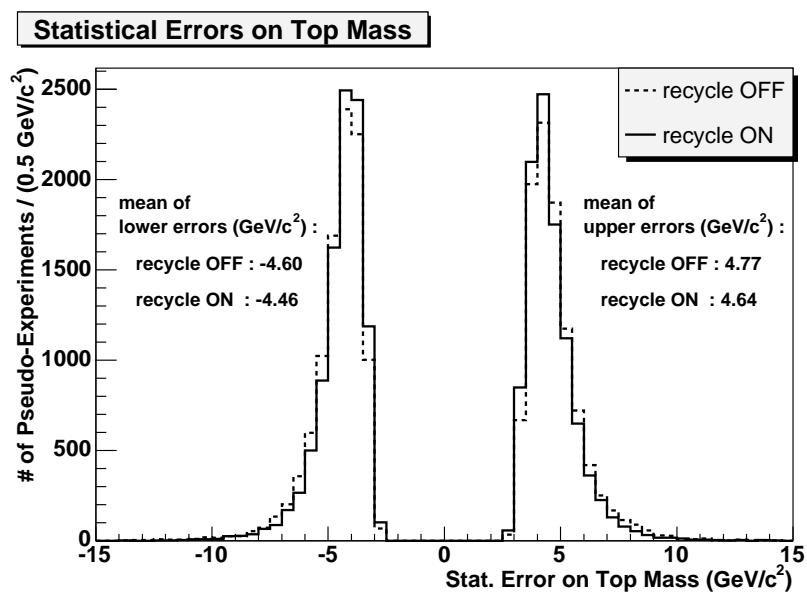

Figure 8.10: Upper and lower statistical error on measured top quark mass in pseudoexperiments for with and without the event recycling. Mean values for upper and lower errors are also shown.

expect a smaller statistical uncertainty when the recycling is enabled. The improvement is of the order of $\sim 3 \%$. 


\section{Chapter 9}

\section{Validation of the Measurement Method}

It is very important to check the validity of the measurement method. It is especially crucial to understand the possible bias to the top quark mass measured in our method. In fact, it is possible that we need to introduce a new function to project the result of the likelihood fitting to the true mass of the top quark. At the same time, we need to assure the validity of our estimation of the statistical error. In this chapter, we perform a validation of our method, by performing a series of pseudo-experiments. The pseudo-experiments were performed with the total number of candidate events in each sample fixed to the number observed in data. The cut on the Jet Probability is set to 0.05 , based on the study described in the previous chapter.

\subsection{Bias to the Center Value}

In this section, we compare the top mass input to the pseudo-experiments and the top mass output from the likelihood fitting, in order to understand the possible bias of our method. Figure 9.1 shows the distribution of the top quark mass (center value) output from the for the combined likelihood fitting with 0,1 and 2 tag samples in 1000 pseudo-experiments, assuming the top mass of $175 \mathrm{GeV} / c^{2}$. We take the median of the distribution as the representative value for the output center values, and estimated the error on the median with :

$$
\sigma_{\text {median }}=\frac{R M S}{\sqrt{N_{P E}}}
$$

where $R M S$ is the RMS of the distribution and $N_{P E}$ is the number of pseudo-experiments (1000 in this case).

We performed a set of 1000 pseudo-experiments with different top mass settings between 150 and $205 \mathrm{GeV} / c^{2}$. The output center value is plotted as a function of the top quark mass input to the pseudo-experiments in Figure 9.2. We observe the general trend that the output is 


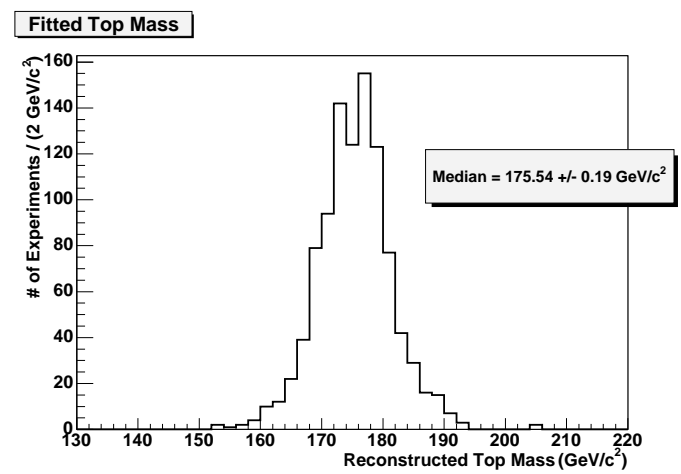

Figure 9.1: Center value returned from the likelihood fitter in pseudo-experiments assuming the top mass of $175 \mathrm{GeV} / c^{2}$.

consistent with the input with these plots.

In order to check the bias more closely, we plot the difference between the output and the input masses against the input mass (Figure 9.3). The bias is not apparent in 2 tag sample and combined measurement at this statistics level. 0 and 1 tag channels look to have certain structures (0 tag channel looks to have a slope. On the other hand 1 tag looks to be shifted toward smaller output mass by $\sim 0.5 \mathrm{GeV} / \mathrm{c}^{2}$ ).

In our attempts to understand the source of this bias, we found that the initial $m_{t o p}$ value for the fitter affects the result. We normally set $m_{t o p}=175 \mathrm{GeV} / c^{2}$ as the initial value in the likelihood fitting. The bias disappears when we use the $m_{t o p}$ value input to the pseudoexperiments as the initial value in the fitting. We also found the following characteristics of this bias :

- This bias disappears if we increase the number of generated events in each pseudo experiment. We expect the bias to disappear with further increased accumulated data.

- The bias disappears with smaller background settings in the pseudo experiments. This is consistent with what we observe in the comparison of 0 tag and 2 tag channels.

A possible understanding of this is that the likelihood function more frequently has a local minimum besides the minimum around the true $m_{t o p}$ as the background contamination grows in a limited statistical situation. In such a case, if we do not input an appropriate initial value for $m_{t o p}$, the fit is likely to end up with the wrong local minimum. However, naively starting with the $m_{t o p}$ value input to the pseudo experiment as the initial parameter value in the fit is obviously not a fair method since we do not know the true top mass in the measurement using data. In order to reduce the bias in the current measurement conditions of 0 and 1 tag channels which are more subject to larger bias, we decided to use the center value obtained in the fitting of the $2 \mathrm{tag}$ channel as the initial value of $m_{\text {top }}$, supposing 2 tag channel is more likely 

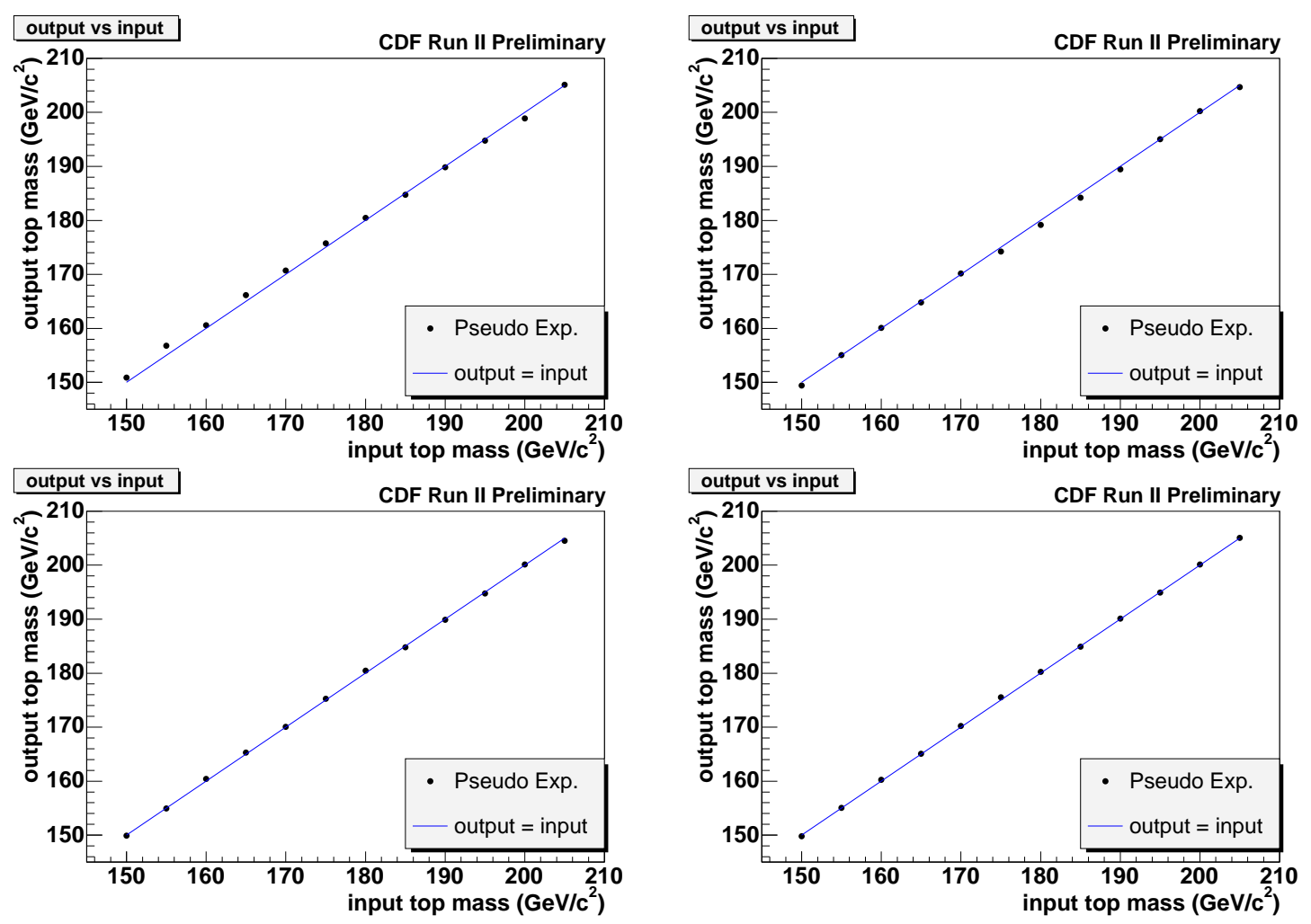

Figure 9.2: Fitted output mass as a function of the top mass input for 0 tag (top-left), 1 tag (topright), 2 tag (bottom-left) and combined fit (bottom-right). The line shows the output = input.

to reconstruct the $m_{t}$ better (pseudo experiments presented in this section already adopts this scheme). Although our study lives with this bias due to the limited statistics in 0 and 1 tag samples, the 2 tag channel is highly purified against the background and is not subject to this bias as illustrated in Figure 9.3. Note that the combined result is also free from this bias, which indicates that the local minima described above disappears by combining the likelihoods of the three channels.

\subsection{Bias to the Statistical Error}

Another important issue here is to know whether the statistical error estimated in the likelihood fit is a valid value. We calculate the pull of the measured top quark mass in each pseudoexperiment in order to check this issue.

$$
p u l l=\frac{\left(m_{t o p}^{\text {output }}-m_{\text {top }}^{\text {input }}\right)}{\sigma_{m_{\text {top }}^{\text {output }}}}
$$



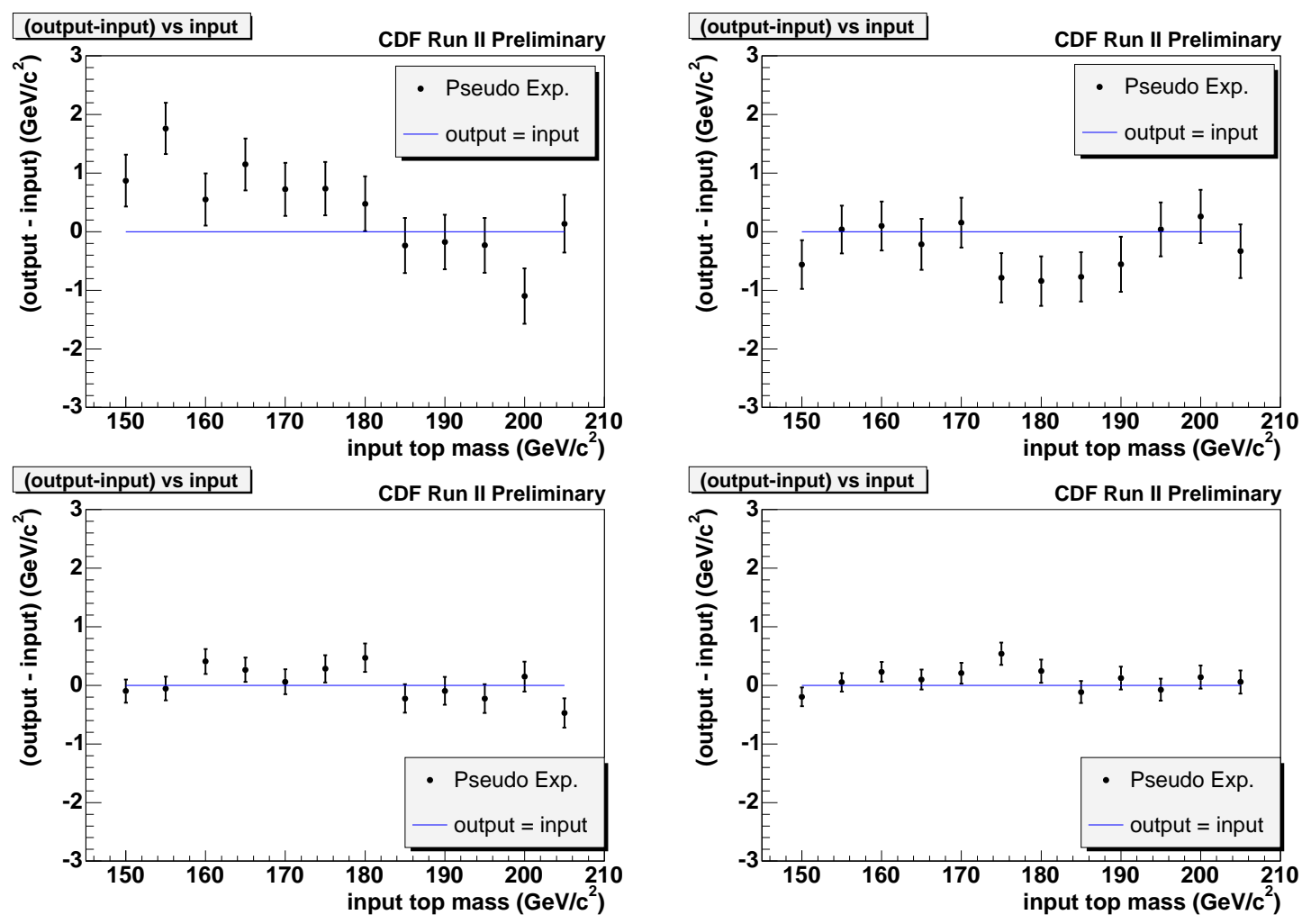

Figure 9.3: Difference between the output and the input masses is plotted against the input top mass for 0 (top-left), 1 (top-right), 2 tag sample (bottom-left) and combined fit (bottom-right). The line shows the output = input.

where $m_{t o p}^{\text {input }}$ and $m_{t o p}^{\text {output }}$ are the input and output top mass of the pseudo-experiment, and $\sigma_{m_{\text {top }}^{\text {output }}}$ is the statistical error on $m_{\text {top }}^{\text {output }}$ estimated in the likelihood fit. In fact, upper and lower errors are independently estimated in each pseudo-experiment. Therefore, we assign the upper error to $\sigma_{m_{\text {top }}^{\text {out }}}$ when $m_{\text {top }}^{\text {output }}>m_{\text {top }}^{\text {input }}$, and the lower error is assigned to $\sigma_{m_{\text {top }}^{\text {output }}}$ when $m_{\text {top }}^{\text {output }}<m_{\text {top }}^{\text {input }}$ in the calculation of the pull. Figure 9.4 shows the distribution of the pull obtained from 1000 pseudo-experiments assuming the mass of the top quark of $175 \mathrm{GeV} / c^{2}$. The distribution of the pull is expected to be centered around 0 and have a width of 1 , if both the center value and the error obtained in the likelihood fit has no bias. We fit the distribution to a Gaussian function, and take the parameters corresponding to the mean and the width of this function to represent the distribution.

We use the same series of pseudo-experiments as in Section 9.1 in this study. Figure 9.5 shows the mean of the pull plotted against the top quark mass to input the pseudo-experiments. This is actually an expected behavior, since the mean of the pull distribution is closely correlated to the difference between the output and input, which is plotted in Figure 9.3.

Finally we plot the width of the pull distribution as a function of the input top quark mass in Figure 9.6. It is clear that the width of the pull is off from the expected value of 1 by more 


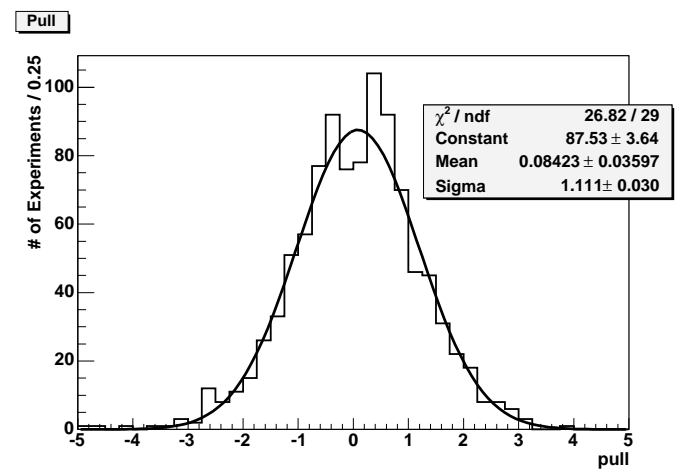

Figure 9.4: Pull in pseudo-experiments assuming the top mass of $175 \mathrm{GeV} / c^{2}$. A fit to Gaussian function is also shown.

than $10 \%$ in 0 sample, and by about $5 \%$ in 1 tag sample. For 2 tag sample and the combined measurement, the deviation from 1 is not so clear, but there might be a few $\%$ of bias. The fact that the width of the pull is larger than 1 indicates that the statistical error estimated in the likelihood fit is an underestimate.

Our investigation shows that this deviation of the pull width from 1 disappears when we a much larger number of events in each sample. In this sense, this is due to the small statistics of the sample used in the measurement of the mass of the top quark.

Although the bias here is of few $\%$ for 2 tag sample and the combined measurement, we correct the statistical error obtained in the likelihood fit by introducing a scale factor in the analysis of data, which will be described in Section 11.6. 

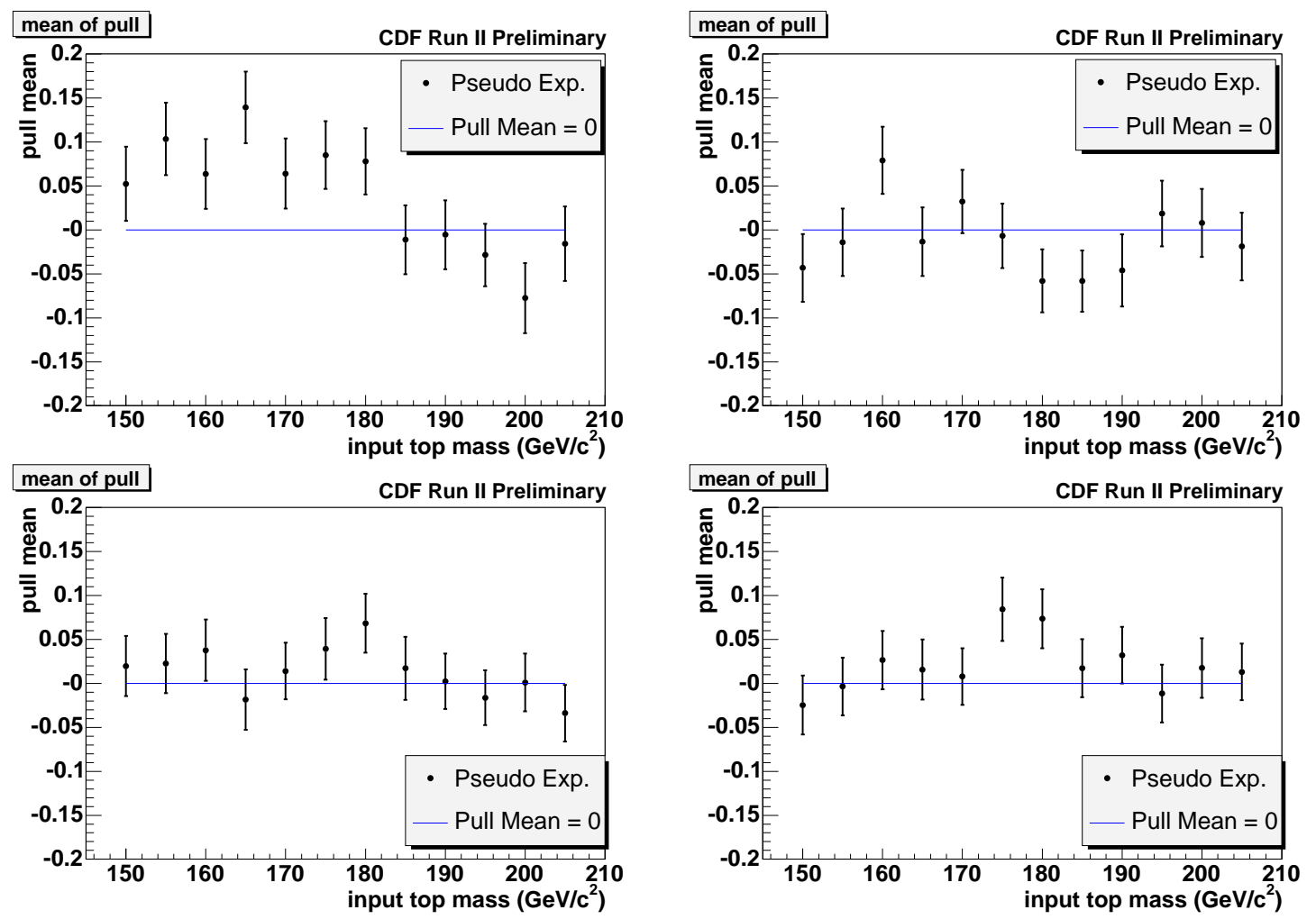

Figure 9.5: The mean of the pull distribution as a function of the input top mass for 0 (top-left), 1 (top-right), 2 tag sample (bottom-left) and combined fit (bottom-right). 

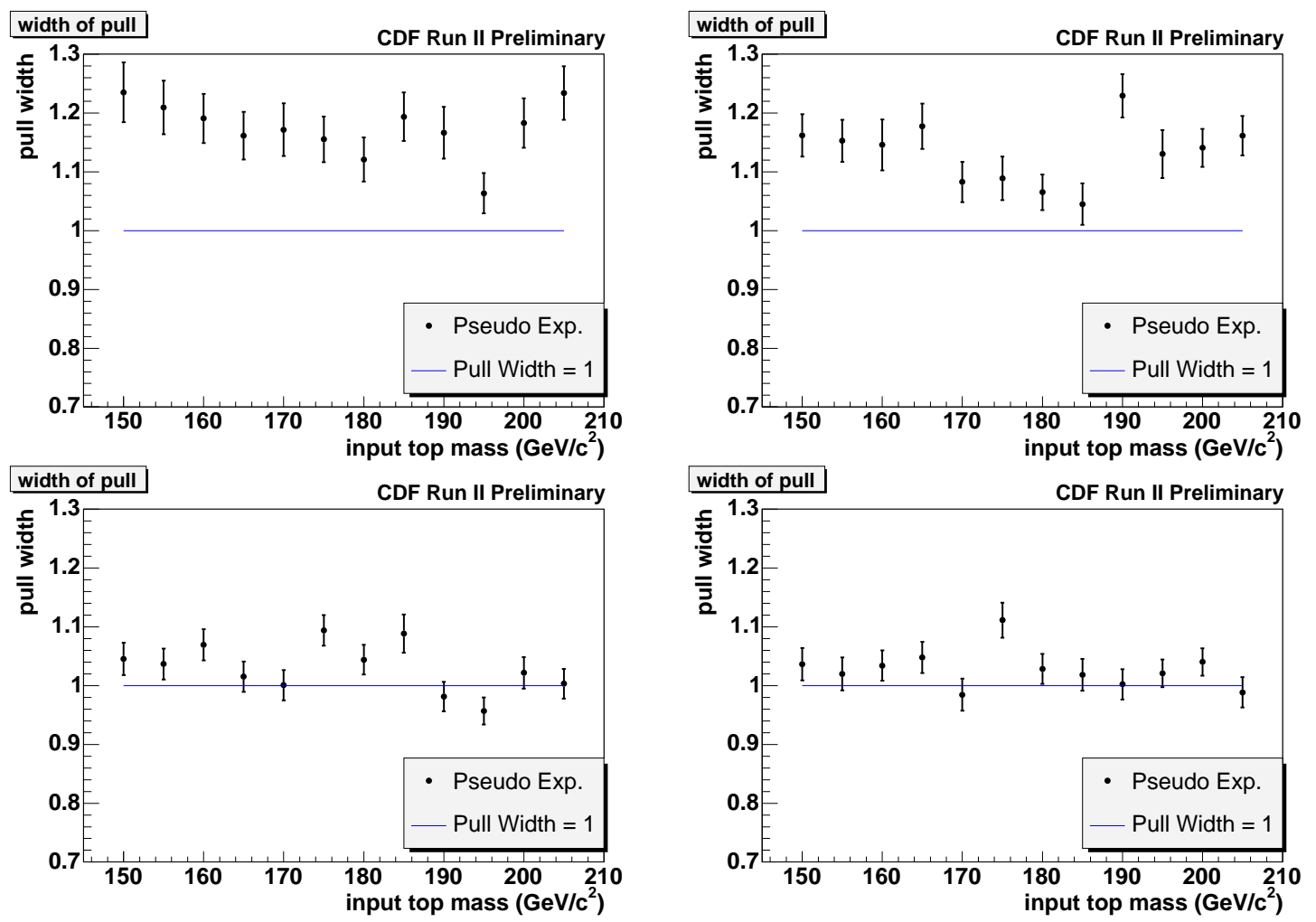

Figure 9.6: Width of the pull distribution as a function of the input top mass for 0 (top-left), 1 (top-right), 2 tag sample (bottom-left) and combined fit (bottom-right). 


\section{Chapter 10}

\section{Systematic Uncertainties}

We estimate the systematic uncertainty on our top mass measurement in this chapter. The systematic uncertainty arises due to our limited understanding of the physical processes which are related to the measurement method. We estimate the systematic uncertainty by breaking it down into different uncertainty sources, which are considered to be independent. The sources considered are :

- generator modeling

- initial state radiation

- final state radiation

- parton distribution function

- $b$-tagging efficiency

- background shape

- resolution of the jet measurement

- jet energy scale

For each source of the systematic uncertainty, we perform a set of pseudo-experiments for an alternative modeling, and assign the difference of the result from that assuming our nominal model as the uncertainty arising from the source.

\subsection{Scheme of Estimation}

In this section we briefly illustrate how we estimate the uncertainty arising from each uncertainty source with an example of the case of generator modeling in 2 tag sample. 
We have been used Monte Carlo events generated by HERWIG assuming it describes the properties of $t \bar{t}$ events well. In order to estimate the uncertainty coming from this modeling, we take the PYTHIA Monte Carlo generator as an alternative modeling and compare results with Monte Carlo sample generated by PYTHIA to those of HERWIG.

Shown in the left plot in Figure 10.1 is a comparison of the signal templates of 2 tag sample produced with Monte Carlo events of PYTHIA and HERWIG. Both Monte Carlo samples were
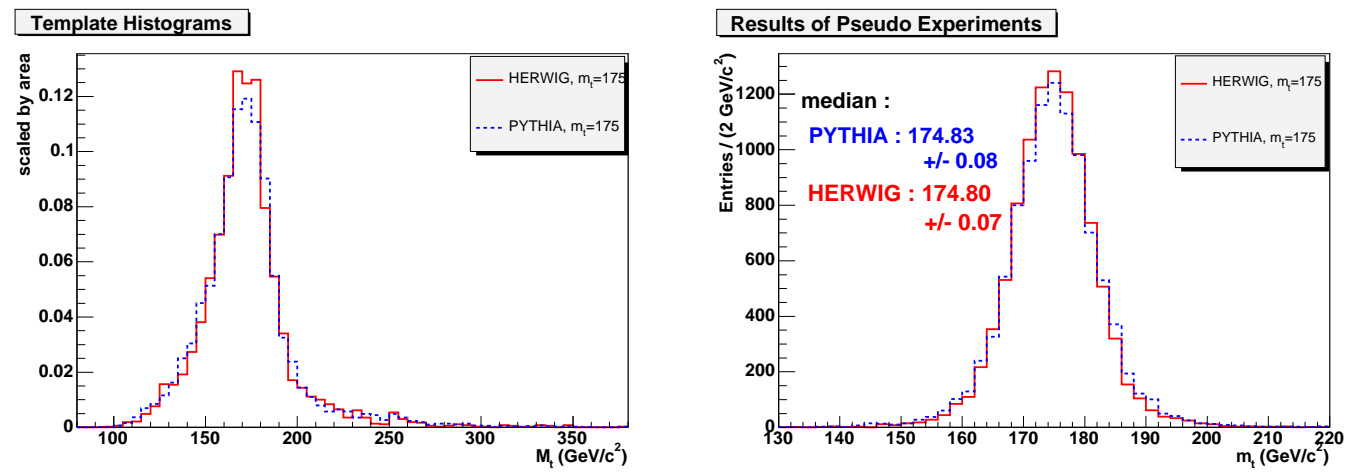

Figure 10.1: Signal template constructed with HERWIG and PYTHIA Monte Carlo events for 2 tag sample (left), and the measured top quark mass in pseudo-experiments by generating pseudo-events due to this template (right).

generated with the assumed mass of the top quark of $175 \mathrm{GeV} / c^{2}$.

Based on these templates, we performed 10000 pseudo-experiments. We used the background template that we usually use. We will estimate the systematic uncertainty arising from background template modeling in another series of pseudo-experiments. The total number of events in each pseudo-experiment was set to the number observed in data. The distribution of the measured top quark mass in such pseudo-experiments for each Monte Carlo modeling is shown in the right plot of Figure 10.1. For each modeling, we calculate the median of the distribution. $R M S / \sqrt{N_{P E}}$ is assigned as error, where $N_{P E}$ is the number of pseudo-experiments. The median was $174.83 \pm 0.08 \mathrm{GeV} / c^{2}$ for PYTHIA, and $174.80 \pm 0.07 \mathrm{GeV} / c^{2}$ for HERWIG. We assign the difference of the two results $0.03 \pm 0.10 \mathrm{GeV} / c^{2}$ as the uncertainty coming from the generator modeling for the measurement in this sample.

\subsection{Generator}

We use Monte Carlo sample generated by HERWIG as our standard Monte Carlo sample. We compared the PYTHIA sample to HERWIG sample. Main difference between these two generators are

- $t \bar{t}$ spin correlation is considered by HERWIG, but not by PYTHIA. 
- Final QED radiation is considered by PYTHIA but not by HERWIG.

- They use different fragmentation models.

Estimated uncertainty using pseudo-experiments is shown in Table 10.1.

\begin{tabular}{|c|c|c|c|c|}
\hline \hline generator & combined & 0 tag & 1 tag & 2 tag \\
\hline pythia & $174.70 \pm 0.06$ & $174.16 \pm 0.15$ & $173.70 \pm 0.13$ & $174.83 \pm 0.08$ \\
herwig & $174.81 \pm 0.06$ & $174.63 \pm 0.14$ & $174.22 \pm 0.14$ & $174.80 \pm 0.07$ \\
\hline$\Delta m_{\text {top }}$ & $0.11 \pm 0.08$ & $0.47 \pm 0.20$ & $0.52 \pm 0.19$ & $0.03 \pm 0.10$ \\
\hline \hline
\end{tabular}

Table 10.1: Estimation of systematic uncertainty due to the generator modeling.

\subsection{Jet Resolution}

The detector simulation applied to Monte Carlo events does not model the jet resolution well. The resolution of the jet energy measurement has been studied by looking at the distribution of $\left(E_{T}^{j e t}-E_{T}^{\gamma}\right) / E_{T}^{\gamma}$ in events where a photon and a jet are identified. The resolution of the energy measurement of photons is negligible compared to that of jets. The width of the distribution was found to be about $15 \%$ narrower for Monte Carlo events than that in data [53]. We estimate the possible bias in the top mass measurement due to this difference in resolution by performing pseudo-experiments using templates produced with the energy of the Monte Carlo jets smeared by $15 \%$. Such a set of pseudo-experiments resulted in a shift on the measured top mass of $1.33 \pm 0.21,0.20 \pm 0.11$ and $0.54 \pm 0.11 \mathrm{GeV} / c^{2}$ for 0,1 and 2 tag samples, respectively. The shift for the combined measurement was found to be $0.71 \pm 0.10 \mathrm{GeV} / c^{2}$. We assign the shifts observed here as the uncertainty coming from the jet resolution modeling of the detector simulation.

\subsection{Jet Energy Scale Uncertainty}

Systematic uncertainty due to the jet energy correction dominates the whole systematic uncertainty. There are seven level corrections for the jet energy as follows:

- Level 1 - Detector response correction as a function of pseudo-rapidity.

- Level 2 - Detector stability on central calorimeter.

- Level 3 - Energy scale correction.

- Level 4 - Multiple interaction correction. 
- Level 5 - Absolute energy correction.

- Level 6 - Underlying event correction.

- Level 7 - Out of cone correction.

The $\pm 1 \sigma$ uncertainty to each level of correction has been obtained along with the measurement of the correction factor. We, therefore, estimate the uncertainty from each level and sum the results to obtain the total uncertainty due to the jet energy scale.

In the data sample used in this analysis, there is few multiple interactions in CDF since we have low beam intensity, and this correction effectively provides no contribution. And underlying (spectator interaction part of the $p \bar{p}$ collision) sample also gives us no correction and uncertainty.

The procedure of estimation of the systematic uncertainty coming from each level of correction is the following :

(1) Change the jet energy scale by $\pm 1 \sigma$, and produce a distribution of reconstructed top mass corresponding to $\pm 1 \sigma$ correction. The $\pm 1 \sigma$ shifted templates are prepared both for signal and background.

(2) Using the template obtained above as a base of the pseudo-data, perform set of pseudoexperiments.

(3)Assign the difference of the medians from \pm 1 sigma correction as the systematic error due to the correction level.

In the construction of the template, the event selection is done with the shifted correction scale. Thus the uncertainty estimate includes influence of the energy scale on the event selection.

Table 10.2 summarizes the uncertainty due to each level. As for the level 7 (out of cone) correction, we divide the uncertainty into two parts : for the energy within $0.4<\Delta R<0.7$ ("splash out") and $0.7<\Delta R$ ("splash out") of the jet axis. We have separated uncertainties for data and Monte Carlo from level 1 to 3 since we apply the jet energy correction of these levels to data in a different way from Monte Carlo samples ${ }^{1}$. This is because of the limited reproductivity of the data by the CDF detector simulation ${ }^{2}$.

\subsection{Initial and Final State Gluon Radiation (ISR / FSR)}

Gluon radiation of the quarks in a $t \bar{t}$ event can result in the mis-measurement of the jet energy or a mis-identification of the jet produced in the $t \bar{t}$ decay, which in turn gives rise to a poor

\footnotetext{
${ }^{1}$ Level 2 correction is the correction for the change in the gain of photo-multipliers of calorimeter. This is mainly due to the aging of the device, and no such correction is necessary for the simulation with Monte Carlo samples.

${ }^{2}$ The correction factor is different between data and Monte Carlo. Therefore, applying the jet energy correction factor for data to Monte Carlo events would result in wrong center value. However, we estimate the uncertainty due to data corrections by applying it to Monte Carlo events, considering that taking the difference of the result of $-1 \sigma$ and $+1 \sigma$ correction would cancel the shift in the center value and result in a right estimate at the first order approximation.
} 


\begin{tabular}{|c|c|c|c|c|c|}
\hline Level & Source & combined & $0 \operatorname{tag}\left(\mathrm{GeV} / c^{2}\right)$ & $1 \operatorname{tag}\left(\mathrm{GeV} / c^{2}\right)$ & $2 \operatorname{tag}\left(\mathrm{GeV} c^{2}\right)$ \\
\hline $1(\operatorname{sim})$ & $\eta$ dep. & $2.24 \pm 0.04$ & $3.33 \pm 0.11$ & $2.35 \pm 0.10$ & $1.82 \pm 0.05$ \\
\hline 1 (data) & $\eta$ dep. & $1.77 \pm 0.04$ & $2.98 \pm 0.11$ & $1.61 \pm 0.10$ & $1.39 \pm 0.05$ \\
\hline $2(\operatorname{sim})$ & Cal. Stability & $0.90 \pm 0.04$ & $1.60 \pm 0.10$ & $0.75 \pm 0.10$ & $0.76 \pm 0.05$ \\
\hline $3(\operatorname{sim})$ & Raw Scale & $3.58 \pm 0.04$ & $4.35 \pm 0.11$ & $3.83 \pm 0.10$ & $3.10 \pm 0.05$ \\
\hline 3 (data) & Raw Scale & $2.89 \pm 0.04$ & $3.72 \pm 0.10$ & $2.85 \pm 0.10$ & $2.52 \pm 0.05$ \\
\hline 5 & Absolute energy & $2.36 \pm 0.04$ & $2.85 \pm 0.11$ & $2.55 \pm 0.10$ & $2.14 \pm 0.05$ \\
\hline \multirow[t]{2}{*}{7} & Out of Cone & $1.65 \pm 0.04$ & $1.97 \pm 0.10$ & $1.78 \pm 0.10$ & $1.55 \pm 0.05$ \\
\hline & Splash out & $1.47 \pm 0.04$ & $1.42 \pm 0.11$ & $1.36 \pm 0.10$ & $1.49 \pm 0.05$ \\
\hline \multicolumn{2}{|r|}{ total } & $6.37 \pm 0.11$ & $8.32 \pm 0.30$ & $6.56 \pm 0.28$ & $5.56 \pm 0.14$ \\
\hline
\end{tabular}

Table 10.2: Systematic uncertainty due to the jet energy correction.

reconstruction of the top quark mass in the event. In this sense, a better understanding of the initial and final gluon radiation processes is essential for a better measurement of the mass of the top quark.

A study dedicated to the understanding of the initial gluon radiation has been performed in CDF Run II [65]. In order to disentangle the ISR effect from FSR, the study was performed using Drell-Yann events with $Z / \gamma^{*}$ decaying into leptons $\left(p \bar{p} \rightarrow Z / \gamma^{*} \rightarrow e^{+} e^{-}, \mu^{+} \mu^{-}\right)$. This study takes advantage of the fact that there is no FSR jets in such a Drell-Yann process. Furthermore, the event is produced by the $q \bar{q}$ annihilation process, and the result is expected to describe the properties of ISR in $t \bar{t}$ events ( $85 \%$ by $q \bar{q}$ and $15 \%$ by $g g$ scattering) well. If an ISR occurs in a Drell-Yann event, part of the $P_{T}$ of the system is carried away by the gluon, skewing the $P_{T}$ spectrum of the di-lepton system. The $P_{T}$ spectrum of the di-lepton system was analyzed in terms of the invariant mass of the leptons. PYTHIA was tuned to reproduce the observed spectrum in data, and parameters reproducing the $+1 \sigma$ ("more ISR") and $-1 \sigma$ ("less ISR") condition was found to be $\left(\Lambda_{Q C D}, K-\right.$ factor $)=(0.384,0.5)$ and $(0.100,2.0)$, respectively, where $K$ - factor is the $\mathrm{K}$ factor to the transverse momentum scale of the ISR evolution. The values of our default PYTHIA sample is $(0.250,1.0)$.

Table 10.3 summarizes the median of measured the top quark mass in pseudo-experiments corresponding to the three ISR settings. Since the result of "default" is always smaller than "more" and "less" ISR cases, we assign the maximum difference among the three numbers as the systematic error.

As for the estimation of the systematic uncertainty coming from the final state radiation, the Monte Carlo samples corresponding to "more" and "less" FSR condition is generated with PYTHIA with $\left(\Lambda_{Q C D}, K-\right.$ factor $)=(0.384,0.5)$ and $(0.100,2.0)$, respectively, where $K$-factor here is the $\mathrm{K}$ factor for FSR evolution, and not the same parameter as that in ISR. These are driven by measurements of gluon radiation in $W \rightarrow q \bar{q}^{\prime}$ and $Z \rightarrow q \bar{q}$ processes at LEP experiment 


\begin{tabular}{|c|c|c|c|c|}
\hline \hline & combined & 0 tag & 1 tag & 2 tag \\
\hline more ISR & $175.13 \pm 0.06$ & $175.11 \pm 0.15$ & $174.64 \pm 0.14$ & $175.03 \pm 0.08$ \\
default & $174.70 \pm 0.06$ & $174.16 \pm 0.15$ & $173.70 \pm 0.13$ & $174.83 \pm 0.08$ \\
less ISR & $175.31 \pm 0.06$ & $175.05 \pm 0.14$ & $174.70 \pm 0.13$ & $175.52 \pm 0.08$ \\
$\Delta m_{\text {top }}$ & $0.61 \pm 0.09$ & $0.95 \pm 0.21$ & $1.00 \pm 0.19$ & $0.69 \pm 0.11$ \\
\hline \hline
\end{tabular}

Table 10.3: Systematic uncertainty due to the initial state gluon radiation.

[66]. Table 10.4 summarizes the results of the pseudo-experiments for "more", "default" and "less" FSR conditions. As in the case of ISR, we take the maximum difference among the three

\begin{tabular}{|c|c|c|c|c|}
\hline \hline & combined & 0 tag & 1 tag & 2 tag \\
\hline more FSR & $175.70 \pm 0.06$ & $174.99 \pm 0.15$ & $174.80 \pm 0.14$ & $176.03 \pm 0.08$ \\
default & $174.70 \pm 0.06$ & $174.16 \pm 0.15$ & $173.70 \pm 0.13$ & $174.83 \pm 0.08$ \\
less FSR & $175.15 \pm 0.06$ & $175.18 \pm 0.14$ & $175.00 \pm 0.14$ & $175.00 \pm 0.08$ \\
\hline$\Delta m_{\text {top }}$ & $1.00 \pm 0.08$ & $1.02 \pm 0.21$ & $1.30 \pm 0.20$ & $1.20 \pm 0.11$ \\
\hline \hline
\end{tabular}

Table 10.4: Systematic uncertainty due to the and final state radiation.

conditions as the uncertainty due to this source.

\subsection{Parton Distribution Functions (PDF)}

Our default $t \bar{t}$ Monte Carlo sample is based on CTEQ5L [48] with $\alpha_{S}=0.118$. We calculate the systematic uncertainty due to PDFs by changing the choice of PDF group, $\alpha_{S}$ and the internal parameters of the PDF.

We generated forty-six reconstructed mass templates, where the PDFs used are :

o - CTEQ5L Our default pdf.

1 - MRST with $\Lambda_{Q C D}=228 \mathrm{MeV}$ Another leading order PDF using more or less the same data as our default but fitted by different group. No significant difference is expected relative to the default, but if a difference is found, we take the difference between CTEQ5 as a systematic due to different PDF group.

2 - MRST with $\Lambda_{Q C D}=300 \mathrm{MeV}$ Same as previous, but using different value of $\alpha_{S}$. The difference between these two is taken as a systematic due to different $\alpha_{S}$.

3 - CTEQ6L More recent fit from CTEQ group for reference. Leading order.

4 - CTEQ6L1 Same as CTEQ6L, but with different $\alpha_{S}$. Also for reference. 
5 - CTEQ6M A next-to-leading order PDF from the CTEQ group. Using a leading order matrix element in conjunction with this PDF does not give a correct description of event rates. However, we assume that the relative behavior of various NLO PDFs is accurately modeled even though we use a LO matrix element.

6-45 - CTEQ6M uncertainty PDFs With the PDF set CTEQ6M, the CTEQ group made available 40 complementary $\mathrm{PDF}$ sets, each of which represents a $\pm 1 \sigma$ variation along one of the twenty eigen vectors (corresponding to 20 parameters of the PDF). $+1 \sigma(-1 \sigma)$ variation on the $n$-th eigen vector comes in $2 n+4$-th and $2 n+5$-th set of our numbering.

The variation of the measured top quark mass due to the different PDF is estimated in pseudo-experiments as usual. Figure 10.2 summarizes the results of the forty-six sets of pseudoexperiments. On the left plots, results from $\pm 1 \sigma$ variation on the same eigen vector is connected with lines.

We sum up half the top mass difference due to each eigenvector $\pm 1 \sigma$ fluctuation in quadrature and obtained the result shown in Table. 10.5

\begin{tabular}{|c|c|c|c|c|}
\hline \hline & combined & 0 tag & 1 tag & 2 tag \\
\hline different family & $0.01 \pm 0.04$ & $0.03 \pm 0.10$ & $0.08 \pm 0.09$ & $0.03 \pm 0.05$ \\
different $\alpha_{S}$ & $0.07 \pm 0.04$ & $0.30 \pm 0.10$ & $0.28 \pm 0.09$ & $0.03 \pm 0.05$ \\
Eigen Vectors & $0.18 \pm 0.10$ & $0.46 \pm 0.22$ & $0.23 \pm 0.19$ & $0.14 \pm 0.11$ \\
\hline total & $0.19 \pm 0.12$ & $0.55 \pm 0.26$ & $0.37 \pm 0.23$ & $0.15 \pm 0.13$ \\
\hline \hline
\end{tabular}

Table 10.5: The estimation of the uncertainty due to PDF.

\subsection{Background Shape}

There are two potential sources for this uncertainty: one is due to the fraction of each background since we fix the fraction of each background process in the background template. The other is due to the $Q^{2}$ dependence of the background shape. Checking with samples with different $Q^{2}$ provides the uncertainty.

To estimate the uncertainty due to the relative background fraction, we performed pseudoexperiments based on the reconstructed top mass distribution of each background process instead of using the combined background for tagged samples. This is an extreme because we do not expect any one of the background processes has the fraction of $100 \%$ in the background template, and must be a safe way of estimating the uncertainty. Table 10.6 summarizes the top mass shifts due to this background shape modeling. We regard half the largest difference among these as the systematic uncertainty. The 0 tag sample measurement is not expected to be shifted due to the variation in the tagged background shape, and the result is consistent with 0 . For the 0 tagged 

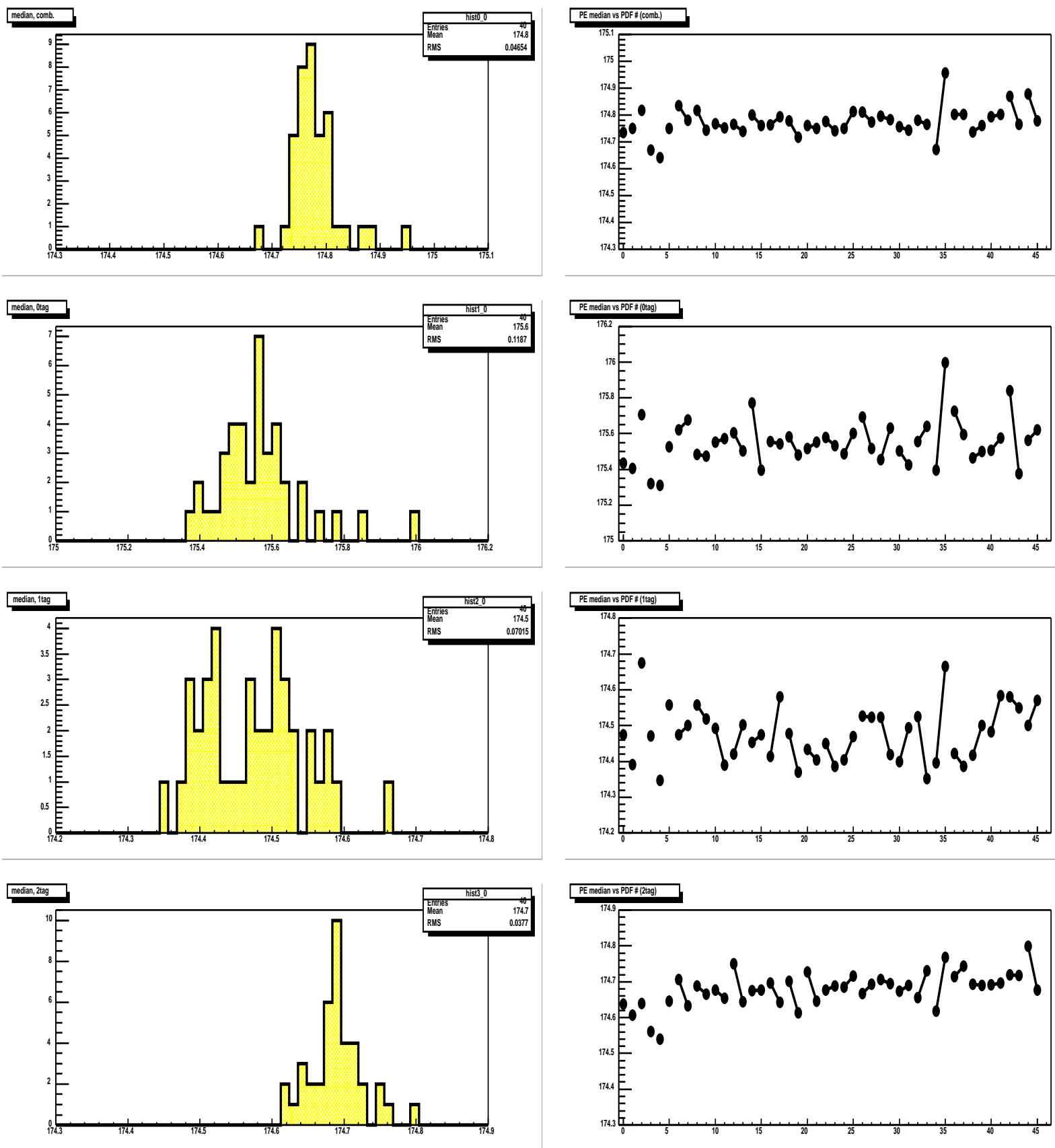

Figure 10.2: The size of the uncertainty due to different $\alpha_{S}$, different PDF groups, and uncertainty in the CTEQ eigenvectors (left plots). The measured top quark mass in pseudoexperiments is plotted as a function of the PDF number defined in text (right plots). Plots are for combined fit, 0,1 and 2 tag samples from top. 
background, we only consider the shape of $W+$ light flavor jets process for the background, and no uncertainty of this type is estimated due to the composition of the 0 tag background template.

\begin{tabular}{|c|c|c|c|c|}
\hline \hline condition & combined & 0 tag & 1 tag & 2 tag \\
\hline combined (default) & $0.00 \pm 0.08$ & $0.00 \pm 0.20$ & $0.00 \pm 0.19$ & $0.00 \pm 0.10$ \\
mistag & $0.02 \pm 0.08$ & $-0.20 \pm 0.20$ & $1.20 \pm 0.19$ & $-0.00 \pm 0.10$ \\
$W b \bar{b}$ & $-0.50 \pm 0.08$ & $0.02 \pm 0.20$ & $-2.34 \pm 0.19$ & $-0.15 \pm 0.10$ \\
single top (s-chan) & $-0.09 \pm 0.08$ & $-0.34 \pm 0.20$ & $0.31 \pm 0.19$ & $0.00 \pm 0.10$ \\
single top (t-chan) & $-0.09 \pm 0.08$ & $-0.11 \pm 0.20$ & $0.00 \pm 0.18$ & $-0.02 \pm 0.10$ \\
\hline$\frac{\Delta \text { mtop }}{2}$ & $0.26 \pm 0.06$ & $0.18 \pm 0.14$ & $1.77 \pm 0.14$ & $0.08 \pm 0.07$ \\
\hline \hline
\end{tabular}

Table 10.6: Systematic uncertainty due to the background fraction of each source.

Four different $Q^{2}$ samples of the $W b \bar{b}+1$ parton Monte Carlo events (our default is $W b \bar{b}+$ 2parton) are used to estimate the $Q^{2}$ uncertainty due to the tagged channels. Half the maximum difference among the results with the four different $Q^{2}$ is taken as the uncertainty of this source. The samples and resulting top mass shifts are shown in Table 10.7.

\begin{tabular}{|c|c|c|c|c|}
\hline \hline condition & combined & 0 tag & 1 tag & 2 tag \\
\hline$Q^{2}=4 M_{W}^{2}$ & $174.76 \pm 0.05$ & $174.56 \pm 0.14$ & $175.01 \pm 0.14$ & $174.67 \pm 0.07$ \\
$Q^{2}=M_{W}^{2}+P_{T}^{w 2}$ & $174.77 \pm 0.05$ & $174.52 \pm 0.14$ & $174.95 \pm 0.14$ & $174.71 \pm 0.07$ \\
$Q^{2}=M_{W}^{2} / 4$ & $174.73 \pm 0.05$ & $174.55 \pm 0.14$ & $174.17 \pm 0.14$ & $174.76 \pm 0.07$ \\
$Q^{2}=M_{W}^{2}$ & $174.78 \pm 0.06$ & $174.48 \pm 0.14$ & $174.59 \pm 0.14$ & $174.70 \pm 0.07$ \\
\hline$\frac{\Delta m_{\text {top }}}{2}$ & $0.03 \pm 0.04$ & $0.04 \pm 0.10$ & $0.42 \pm 0.10$ & $0.05 \pm 0.05$ \\
\hline
\end{tabular}

Table 10.7: Top mass results in pseudo-experiments assuming different $Q^{2}$ for $W b \bar{b}$ background process in the construction of background template.

For the 0tag background, we estimate the uncertainty by varying the $Q^{2}$ for Monte Carlo samples with different $Q^{2}$ and and the parton multiplicity. We used VECBOS $W+3$ parton (with hadronization and fragmentation by HERWIG, the basic function of this Monte Carlo generator is the same as ALPGEN) sample for the the comparison of different $Q^{2}$. On the other hand, we used ALPGEN $W+3$ partons and $W+4$ partons samples for the comparison of Monte Carlo samples with different parton multiplicities. The shift in the measured top quark mass in pseudo-experiments due to each variation in the Monte Carlo modeling is added in quadrature and we obtained the total uncertainty due to this source (summarized in Table 10.8).

Although we independently estimate the uncertainty due to the background shape modeling of 0 tag and tagged samples, we included all the channels in the tables. We observe 


\begin{tabular}{|c|c|c|c|c|}
\hline \hline condition & combined & 0 tag & 1 tag & 2 tag \\
\hline$\left(Q^{2}=m_{W}^{2}\right)-\left(Q^{2}=<p t_{j e t}>^{2}\right)$ & $-0.11 \pm 0.08$ & $-1.02 \pm 0.22$ & $0.07 \pm 0.19$ & $-0.04 \pm 0.10$ \\
parton multiplicity & $0.62 \pm 0.08$ & $2.62 \pm 0.21$ & $0.25 \pm 0.19$ & $0.14 \pm 0.10$ \\
\hline total & $0.63 \pm 0.11$ & $2.81 \pm 0.30$ & $0.26 \pm 0.27$ & $0.15 \pm 0.14$ \\
\hline \hline
\end{tabular}

Table 10.8: Top mass shift due to different 0 tag background templates.

zero-consistent shifts in measured top mass in the channels irrelevant to the variation in the background templates as expected. Quadratically adding the results from Tables 10.6, 10.7 and 10.8 we obtained the total estimated systematic uncertainty due to the background shape as summarized in Table 10.9 .

\begin{tabular}{|c|c|c|c|c|}
\hline \hline & combined & 0 tag & 1 tag & 2 tag \\
\hline bkgd shape total & $0.68 \pm 0.13$ & $2.82 \pm 0.35$ & $1.84 \pm 0.32$ & $0.17 \pm 0.16$ \\
\hline \hline
\end{tabular}

Table 10.9: Total uncertainty due to background shape modeling.

\section{$10.8 \quad b$-Tagging Scale Factor}

We only discuss uncertainty due to the scale factors of SECVTX and Jet Probability since mistag uncertainties were already discussed in the Subsection 6.2.1. We usually apply the nominal data-to-Monte Carlo scale factor for absolute value of of the $b$-tagging efficiency in our study. However, the scale factor measurement comes with an uncertainty, and we have to consider the $\pm 1 \sigma$ variation of the scale factor. At the same time, there is no reason to not consider the possible $E_{T}^{j e t}$ dependence of the scale factor.

The scale factors of the $b$-taggers $[56,44]$ were measured to be $0.82 \pm 0.06$ for SECVTX and $0.820 \pm 0.095$ for Jet Probability. The $E_{T}$ dependence corresponding to $\pm 1 \sigma$ were found to be :

$$
\begin{array}{rc}
\operatorname{SECVTX}(+1 \sigma) & : 0.81+0.0011 \times\left(E_{T}-25 \mathrm{GeV}\right) \\
\operatorname{SECVTX}(-1 \sigma) & : 0.81-0.0003 \times\left(E_{T}-25 \mathrm{GeV}\right) \\
\text { Jet Probability }(+1 \sigma) & : 0.820+0.0012 \times\left(E_{T}-20 \mathrm{GeV}\right) \\
\text { Jet Probability }(-1 \sigma) & : 0.820-0.0007 \times\left(E_{T}-20 \mathrm{GeV}\right)
\end{array}
$$

Since we use two different $b$-tagging algorithms in our analysis, we have to consider the scale factors for both, taking into account the correlation between these two taggers. However, no study on such correlation has been performed yet, and we consider extreme cases : (1) no correlation at all, (2) $100 \%$ positive correlation and (3) $100 \%$ negative correlation. The estimation must be a safe one by taking these extreme cases. 
Nine combinations of the following are tested for the absolute value and the $E_{T}$ dependence of the scale factors: SECVTX $(+, 0,-)$ and Jet Probability $(+, 0,-)$, where " \pm " and 0 each denote the $\pm 1 \sigma$ variation and the nominal value for the scale factor. The scale factor is considered into the signal mass template by randomly rejecting a fraction of $b$-tags of jets in the Monte Carlo studies. By changing such fraction of rejection, we compose templates for varied scale factors.

Tables 10.10 and 10.11 summarizes the pseudo-experiment results for nine combinations of varied scale factor conditions for the absolute value and $E_{T}$ dependence, respectively. Half the maximum difference among the nine cases is taken as the uncertainty. The uncertainties due to the absolute value and $E_{T}$ dependence were added in quadrature to estimate the total uncertainty due to the $b$-tagging scale factor (Table 10.12).

\begin{tabular}{|c|c|c|c|c|c|}
\hline \hline SECVTX & Jet Probability & combined & $0 \mathrm{tag}$ & $1 \mathrm{tag}$ & $2 \mathrm{tag}$ \\
\hline 0 & 0 & $174.67 \pm 0.06$ & $175.27 \pm 0.15$ & $174.50 \pm 0.14$ & $174.42 \pm 0.07$ \\
0 & - & $174.60 \pm 0.06$ & $175.16 \pm 0.15$ & $174.43 \pm 0.13$ & $174.59 \pm 0.07$ \\
0 & + & $174.57 \pm 0.06$ & $175.11 \pm 0.15$ & $174.73 \pm 0.14$ & $174.32 \pm 0.07$ \\
- & 0 & $174.62 \pm 0.06$ & $174.82 \pm 0.15$ & $174.83 \pm 0.14$ & $174.50 \pm 0.07$ \\
- & - & $174.73 \pm 0.06$ & $174.98 \pm 0.15$ & $174.65 \pm 0.14$ & $174.72 \pm 0.07$ \\
- & + & $174.54 \pm 0.06$ & $174.95 \pm 0.15$ & $174.69 \pm 0.14$ & $174.45 \pm 0.07$ \\
+ & 0 & $174.59 \pm 0.06$ & $175.04 \pm 0.15$ & $174.56 \pm 0.14$ & $174.53 \pm 0.07$ \\
+ & - & $174.87 \pm 0.06$ & $175.12 \pm 0.15$ & $174.57 \pm 0.14$ & $174.75 \pm 0.07$ \\
+ & + & $174.80 \pm 0.06$ & $175.13 \pm 0.15$ & $174.79 \pm 0.14$ & $174.62 \pm 0.07$ \\
\hline \multicolumn{2}{|l|}{$\frac{\Delta \text { mtop }}{2}$} & $0.17 \pm 0.05$ & $0.22 \pm 0.11$ & $0.20 \pm 0.10$ & $0.20 \pm 0.05$ \\
\hline \hline
\end{tabular}

Table 10.10: Top mass measured in pseudo-experiments supposing nine different combination of varied absolute values of scale factors for the SECVTX and Jet Probability.

\subsection{Summary of the Systematic Uncertainties}

Table 10.13 summarizes the systematic uncertainty due to each source estimated in this chapter. The total systematic uncertainty is obtained by adding all sub-categories in quadrature. The systematic uncertainty in 2 tag sample measurement is smaller than those of the 1 or 0 tag. This is considered to be due to the fact that the reconstruction of 2 tag events does not get as much affected as the other channels in such a way that a correctly reconstructed event turns to be combinatorial background with a varied jet energy correction. 


\begin{tabular}{|c|c|c|c|c|c|}
\hline SECVTX & Jet Probability & combined & 0 tag & $1 \mathrm{tag}$ & $2 \mathrm{tag}$ \\
\hline 0 & 0 & $174.67 \pm 0.06$ & $175.27 \pm 0.15$ & $174.50 \pm 0.14$ & $174.42 \pm 0.07$ \\
\hline 0 & - & $174.53 \pm 0.06$ & $174.94 \pm 0.15$ & $174.56 \pm 0.13$ & $174.26 \pm 0.07$ \\
\hline 0 & + & $174.73 \pm 0.06$ & $175.19 \pm 0.15$ & $174.83 \pm 0.14$ & $174.45 \pm 0.07$ \\
\hline- & 0 & $174.54 \pm 0.06$ & $175.32 \pm 0.15$ & $174.36 \pm 0.14$ & $174.39 \pm 0.07$ \\
\hline- & - & $174.49 \pm 0.06$ & $175.17 \pm 0.15$ & $174.34 \pm 0.13$ & $174.45 \pm 0.07$ \\
\hline- & + & $174.61 \pm 0.06$ & $175.08 \pm 0.15$ & $174.60 \pm 0.13$ & $174.47 \pm 0.07$ \\
\hline+ & 0 & $174.69 \pm 0.06$ & $174.98 \pm 0.15$ & $174.45 \pm 0.14$ & $174.47 \pm 0.07$ \\
\hline+ & - & $174.65 \pm 0.06$ & $175.09 \pm 0.15$ & $174.91 \pm 0.14$ & $174.62 \pm 0.07$ \\
\hline+ & + & $174.64 \pm 0.06$ & $175.18 \pm 0.15$ & $174.67 \pm 0.14$ & $174.50 \pm 0.07$ \\
\hline \multicolumn{2}{|r|}{$\frac{\Delta \text { tmtop }}{2}$} & $0.10 \pm 0.04$ & $0.19 \pm 0.11$ & $0.25 \pm 0.10$ & $0.18 \pm 0.05$ \\
\hline
\end{tabular}

Table 10.11: Top mass measured in pseudo-experiments supposing nine different combination of varied $E_{T}$ dependence of scale factors for the SECVTX and Jet Probability.

\begin{tabular}{|c|c|c|c|c|}
\hline \hline & combined & 0 tag & 1 tag & 2 tag \\
\hline absolute value & $0.17 \pm 0.05$ & $0.22 \pm 0.11$ & $0.20 \pm 0.10$ & $0.20 \pm 0.05$ \\
$E_{T}$ dependence & $0.10 \pm 0.04$ & $0.19 \pm 0.11$ & $0.25 \pm 0.10$ & $0.18 \pm 0.05$ \\
\hline total & $0.20 \pm 0.07$ & $0.29 \pm 0.15$ & $0.32 \pm 0.14$ & $0.27 \pm 0.07$ \\
\hline \hline
\end{tabular}

Table 10.12: Top mass shift on $b$-tag scale factor variations.

\begin{tabular}{|c|c|c|c|c|}
\hline \hline systematic & combined & 0 tag & 1 tag & 2 tag \\
\hline ISR & $0.61 \pm 0.09$ & $0.95 \pm 0.21$ & $1.00 \pm 0.19$ & $0.69 \pm 0.11$ \\
FSR & $1.00 \pm 0.08$ & $1.02 \pm 0.21$ & $1.30 \pm 0.20$ & $1.20 \pm 0.11$ \\
pdf & $0.19 \pm 0.12$ & $0.55 \pm 0.26$ & $0.37 \pm 0.23$ & $0.15 \pm 0.13$ \\
b-tagging & $0.20 \pm 0.07$ & $0.29 \pm 0.15$ & $0.32 \pm 0.14$ & $0.27 \pm 0.07$ \\
bkgd shape total & $0.68 \pm 0.13$ & $2.82 \pm 0.35$ & $1.84 \pm 0.32$ & $0.17 \pm 0.16$ \\
jet resolution & $0.71 \pm 0.10$ & $1.33 \pm 0.21$ & $0.20 \pm 0.11$ & $0.54 \pm 0.11$ \\
jet energy scale & $6.37 \pm 0.11$ & $8.32 \pm 0.30$ & $6.56 \pm 0.28$ & $5.56 \pm 0.14$ \\
generator & $0.11 \pm 0.08$ & $0.47 \pm 0.20$ & $0.52 \pm 0.19$ & $0.03 \pm 0.10$ \\
\hline total & $6.56 \pm 0.28$ & $9.03 \pm 0.69$ & $7.06 \pm 0.61$ & $5.77 \pm 0.34$ \\
\hline \hline
\end{tabular}

Table 10.13: The summary of the systematic uncertainties. We assume these are uncorrelated. 


\section{Chapter 11}

\section{Results and Discussions}

In this chapter we finally analyze the data obtained in the CDF Run II experiment and measure the mass of the top quark using the technique described in the preceding chapters. The integrated luminosity corresponding to the data used in this analysis is :

- $162 \mathrm{pb}^{-1}$ for 1 and 2 tag samples.

- $193 \mathrm{pb}^{-1}$ for 0 tag samples.

\subsection{Candidate Events}

We describe the candidate events found in the data in this section.

Thirty-eight events were selected with at least one SECVTX tagged jet with the kinematic requirements. Seventeen events were found to have another $b$-tagged jet. Six out of seventeen were rejected by the $\chi^{2}$ cut, and no events were failed due to the $m_{j j}$ cut. Six events which unsatisfied the $\chi^{2}$ cut were fed back to the 1 tag sample selection criteria. Table 11.1 shows the details of the remaining eleven events which satisfied all cuts and were used in the mass measurement in the 2 tag sample. Summarized in this table are the run number and event number in the CDF Run II experiment, the type of lepton (CEM : electron, CMUP : muon with $|\eta|<0.6$, CMX : muon with $0.6<|\eta|<1.0), P_{T}$ of the lepton, $E_{T}, E_{T}$ of the leading four jets, $\chi^{2}$ and the reconstructed mass $m_{t}$ and the invariant mass of the two light flavor jets. Table 11.2 summarizes the six events rejected by the $\chi^{2}$ cut.

Twenty-six events were found to fulfill the kinematic and tagging conditions of 1 tag sample, including the six events which failed the $\chi^{2}$ cut of the $2 \mathrm{tag}$ sample selection. Nine out of the twenty-six events were rejected by the $\chi^{2}$ cut, and seventeen events were selected to be used in the top mass measurement in $1 \mathrm{tag}$ sample. Table 11.3 shows the details of the remaining seventeen single tagged events which satisfied all cuts and were used in the mass measurement as well as the nine events rejected by the $\chi^{2}$ cut. 


\begin{tabular}{|rr|rr|rr|rrrr|rrr|}
\hline \hline run & event & $n_{j}$ & $l$ & $\begin{array}{r}p_{t}^{l} \\
\mathrm{GeV}\end{array}$ & $\begin{array}{r}E_{T} \\
\mathrm{GeV}\end{array}$ & $\begin{array}{r}E_{T}^{j 1} \\
\mathrm{GeV}\end{array}$ & $\begin{array}{r}E_{T}^{j 2} \\
\mathrm{GeV}\end{array}$ & $\begin{array}{r}E_{T}^{j 3} \\
\mathrm{GeV}\end{array}$ & $\begin{array}{r}E_{T}^{j 4} \\
\mathrm{GeV}\end{array}$ & $\chi^{2}$ & $\begin{array}{r}m_{t} \\
\mathrm{GeV}\end{array}$ & $\begin{array}{r}m_{j j} \\
\mathrm{GeV}\end{array}$ \\
\hline 144674 & 1782954 & 3.5 & $\mathrm{CEM}$ & 98.7 & 31.6 & 48.5 & $44.1^{J}$ & $40.3^{S}$ & 11.7 & 4.9 & 132.6 & 72.1 \\
153693 & 799494 & 4 & $\mathrm{CMUP}$ & 54.4 & 37.1 & $101.8^{J}$ & 71.0 & $57.4^{S J}$ & 37.2 & 0.4 & 176.8 & 85.7 \\
153694 & 1694029 & 4 & $\mathrm{CEM}$ & 31.8 & 31.5 & $126.4^{J}$ & $63.7^{S J}$ & 39.2 & 25.1 & 5.0 & 154.0 & 66.8 \\
154175 & 1630925 & 4 & $\mathrm{CEM}$ & 30.4 & 115.1 & 122.3 & 31.5 & $18.9^{S}$ & $17.1^{J}$ & 0.8 & 131.6 & 89.8 \\
155145 & 132579 & 3.5 & $\mathrm{CEM}$ & 52.9 & 31.7 & 82.9 & $65.6^{S J}$ & $35.4^{S J}$ & 13.5 & 0.3 & 169.9 & 78.3 \\
160437 & 280173 & 4 & $\mathrm{CMUP}$ & 43.1 & 29.9 & $71.3^{J}$ & $61.9^{S}$ & 51.9 & $18.5^{J}$ & 0.1 & 178.6 & 84.6 \\
161788 & 361577 & 4 & $\mathrm{CMUP}$ & 37.1 & 62.9 & $129.4^{S J}$ & 98.0 & $66.2^{J}$ & 56.1 & 0.7 & 177.1 & 75.2 \\
162986 & 1538897 & 4 & $\mathrm{CEM}$ & 52.2 & 51.7 & $106.5^{J}$ & 65.6 & $46.0^{S J}$ & 32.9 & 1.5 & 264.6 & 91.7 \\
163012 & 2249546 & 4 & $\mathrm{CMUP}$ & 80.2 & 34.5 & $100.6^{J}$ & 55.7 & 31.7 & $26.4^{S J}$ & 6.4 & 192.3 & 62.4 \\
166567 & 11615607 & 4 & $\mathrm{CMUP}$ & 39.7 & 50.1 & $84.1^{S J}$ & $81.6^{S J}$ & 43.4 & 33.1 & 5.6 & 247.0 & 61.5 \\
166614 & 804529 & 4 & $\mathrm{CEM}$ & 111.2 & 29.4 & 103.4 & $53.0^{S J}$ & 49.3 & $24.3^{J}$ & 0.6 & 184.6 & 75.0 \\
\hline \hline
\end{tabular}

Table 11.1: The eleven events in 2 tag sample used in the measurement of the top quark mass. Jets with their $E_{T}$ values tagged with ${ }^{S}$ are SECVTX tagged and with ${ }^{J}$ are tagged by Jet Probability. The jet $E_{T}$ shown here are after the generic correction at level 5 .

\begin{tabular}{|rr|rr|rr|rrrr|rrr|}
\hline \hline run & event & $n_{j}$ & $\mathrm{l}$ & $\begin{array}{r}p_{t}^{l} \\
\mathrm{GeV}\end{array}$ & $\begin{array}{r}\mathscr{E}_{T} \\
\mathrm{GeV}\end{array}$ & $\begin{array}{r}E_{T}^{j 1} \\
\mathrm{GeV}\end{array}$ & $\begin{array}{r}E_{T}^{j 2} \\
\mathrm{GeV}\end{array}$ & $\begin{array}{r}E_{T}^{j 3} \\
\mathrm{GeV}\end{array}$ & $\begin{array}{r}E_{T}^{j 4} \\
\mathrm{GeV}\end{array}$ & $\chi^{2}$ & $\begin{array}{r}m_{t} \\
\mathrm{GeV}\end{array}$ & $\begin{array}{r}m_{j j} \\
\mathrm{GeV}\end{array}$ \\
\hline 148153 & 6088 & 3.5 & $\mathrm{CEM}$ & 54.9 & 48.2 & $71.9^{S}$ & $49.4^{J}$ & 19.2 & 9.4 & 26.1 & 177.1 & 41.9 \\
156116 & 6116596 & 4 & $\mathrm{CEM}$ & 25.3 & 28.2 & $74.5^{S J}$ & $57.1^{S J}$ & $36.6^{J}$ & 29.3 & 366.8 & 231.7 & 20.7 \\
160591 & 894406 & 4 & $\mathrm{CMUP}$ & 114.8 & 110.5 & $156.4^{J}$ & $70.3^{S J}$ & 61.4 & 17.6 & 9.6 & 232.2 & 92.5 \\
165314 & 1155563 & 3.5 & $\mathrm{CMUP}$ & 28.8 & 12.6 & $56.3^{J}$ & $22.8^{S J}$ & 20.7 & 10.4 & 18.6 & 149.4 & 43.2 \\
167139 & 1191211 & 4 & $\mathrm{CMX}$ & 31.2 & 63.5 & $62.5^{S J}$ & $42.3^{J}$ & $40.2^{S J}$ & 23.6 & 13.6 & 175.0 & 56.4 \\
167551 & 7969376 & 4 & $\mathrm{CEM}$ & 77.2 & 54.9 & $79.6^{S J}$ & $50.4^{J}$ & $31.7^{S J}$ & 25.0 & 173.6 & 278.0 & 40.9 \\
\hline \hline
\end{tabular}

Table 11.2: The six events rejected by the $\chi^{2}$ cut in the 2 tag sample selection.

Forty-two events survived our kinematic cuts for the no-tag selection criteria, including the $E_{T}^{j 4}>21 \mathrm{GeV}$. Table 11.4 summarizes the properties of these events. Two of these events were rejected at our $\chi^{2}$ cut, which are shown at the bottom of this table. Remaining forty events are used as 0 tag sample and are used for the mass measurement. There are two events which has SECVTX tags in this 0 tag sample. The operation condition of the silicon detector was considered not sufficient enough in the data taking of these two events, and these events were not categorized into 1 tag sample.

\subsection{Di-jet mass $m_{j j}$ for the Selected 2 Tag Events}

Before measuring the mass of the top quark, it would be interesting to assure that the hadronically decaying $W$ boson is reconstructed as expected. We reconstruct the mass of the $W$ boson 
by calculating the invariant mass of the two jets assigned to the light flavor partons in the $\chi^{2}$ fitter using the events in the 2 tag sample. Energies of the jets after the all the correction (including top-specific correction) are used in the calculation of the invariant mass. We show the invariant mass before and after the $\chi^{2}$ cut in Figure 11.1, with sixteen and eleven events fulfilling the 2 tag sample selection at each stage, respectively. In the figure, distributions obtained using $t \bar{t}$ Monte Carlo sample with the top mass input of $175 \mathrm{GeV} / c^{2}$ are shown for comparison. The histograms of the Monte Carlo events are scaled so that the areas equal to those of data. The
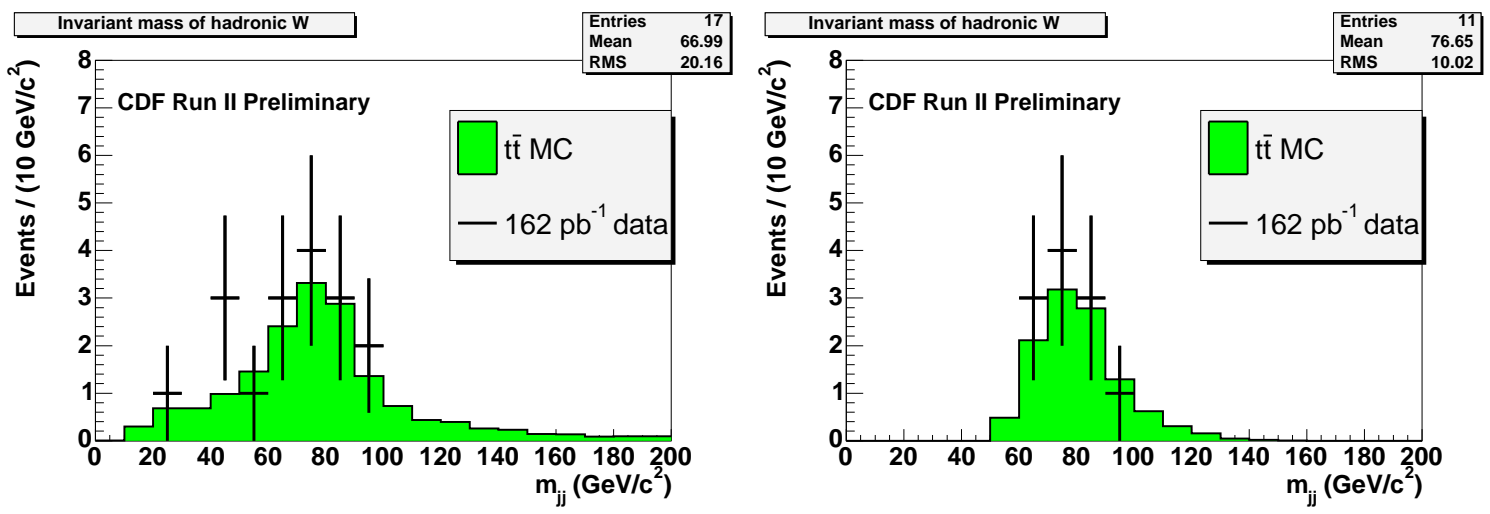

Figure 11.1: Invariant mass of the two light flavor jets before (left) and after (right) the $\chi^{2}$ cut. Histograms show the Monte Carlo distribution $\left(m_{t}=175 \mathrm{GeV} / c^{2}\right)$ while the crosses show the data.

large tails of the distribution before the $\chi^{2}$ cut are due to the wrong combination as well as the initial/final gluon radiation. We can clearly see the effect of the $\chi^{2}$ cut in the figure. These plots show that the properties of our selected candidates are well consistent with the description of $t \bar{t}$ events in the Standard Model.

\subsection{Results of the Likelihood Fit}

Using the selected sixty-eight events the likelihood fit was performed to measure the mass of the top quark. Measurement with the $2 \mathrm{tag}$ sample results in the mass the top quark of $180.9{ }_{-5.8}^{+6.1} \mathrm{GeV} c^{2}$, the 1 tag sample yields $132.8{ }_{-7.4}^{+23.4} \mathrm{GeV} / c^{2}$, and the 0 tag sample gives $176.4_{-7.6}^{+9.3} \mathrm{GeV} / c^{2}$. The combined likelihood fit of these three channels results in $177.2_{-4.6}^{+4.8} \mathrm{GeV} / c^{2}$. Figure 11.2 shows the reconstructed mass distribution of each sample along with the best fit result, as well as the likelihood curve used in extracting the mass of the top quark.

We also tried a likelihood fit to each sample without the constraint on the number of background events. We can obtain another result on the mass of the top quark that is independent of the estimation of the number of background events by performing this fit. This serves as a crosscheck of our result of the likelihood fit with the background constraint. The fit was 

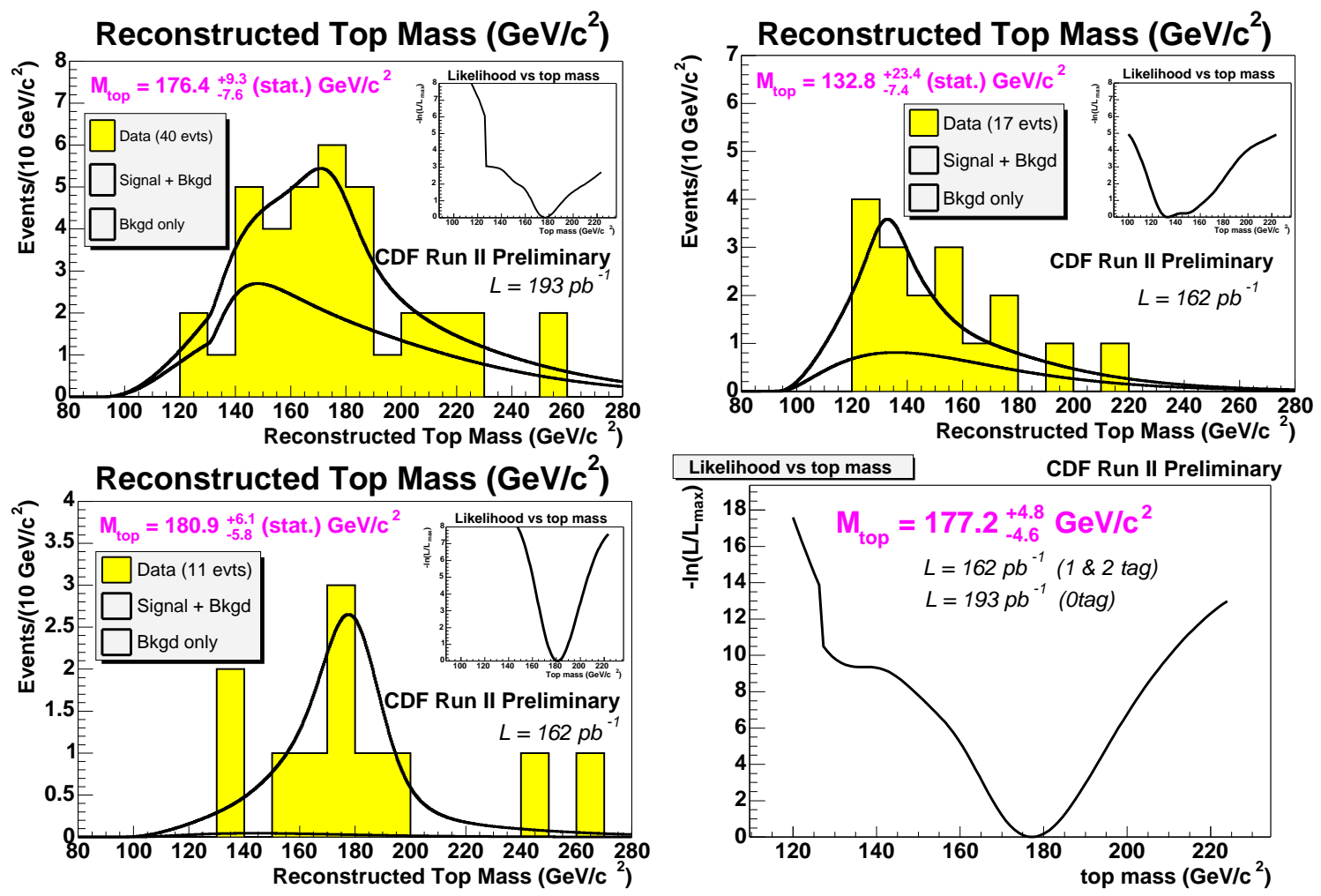

Figure 11.2: Result of the likelihood fit to the data sample for 0 tag (top-left), 1 tag (top-right), $2 \mathrm{tag}$ (bottom-left) and the combined fit of these three channels (bottom-right). The histogram shows the reconstructed mass of the data events for each sample. The distribution shape and the background contribution corresponding to the best fit are shown in lines. In the top-right box of each plot is the likelihood curve used in the measurement of the mass of the top quark. For the combined fit, we only show the likelihood curve. 
performed without the $L_{b k g}$ term in 7.1 :

$$
L=L_{\text {shape }}
$$

The fit mass of the top quark is : $181.9{ }_{-6.4}^{+7.1} \mathrm{GeV} / c^{2}$ for 2 tag sample, $132.6{ }_{-9.0}^{+24.2} \mathrm{GeV} / c^{2}$ for 1 tag sample and $176.1_{-6.7}^{+8.1} \mathrm{GeV} / c^{2}$ for 0 tag sample. The combined fit of these three channels is $179.4_{-5.0}^{+5.4} \mathrm{GeV} / c^{2}$, with the background fraction for 1 tag channel of $100 \%$. Figure 11.3 shows the result of this fit.
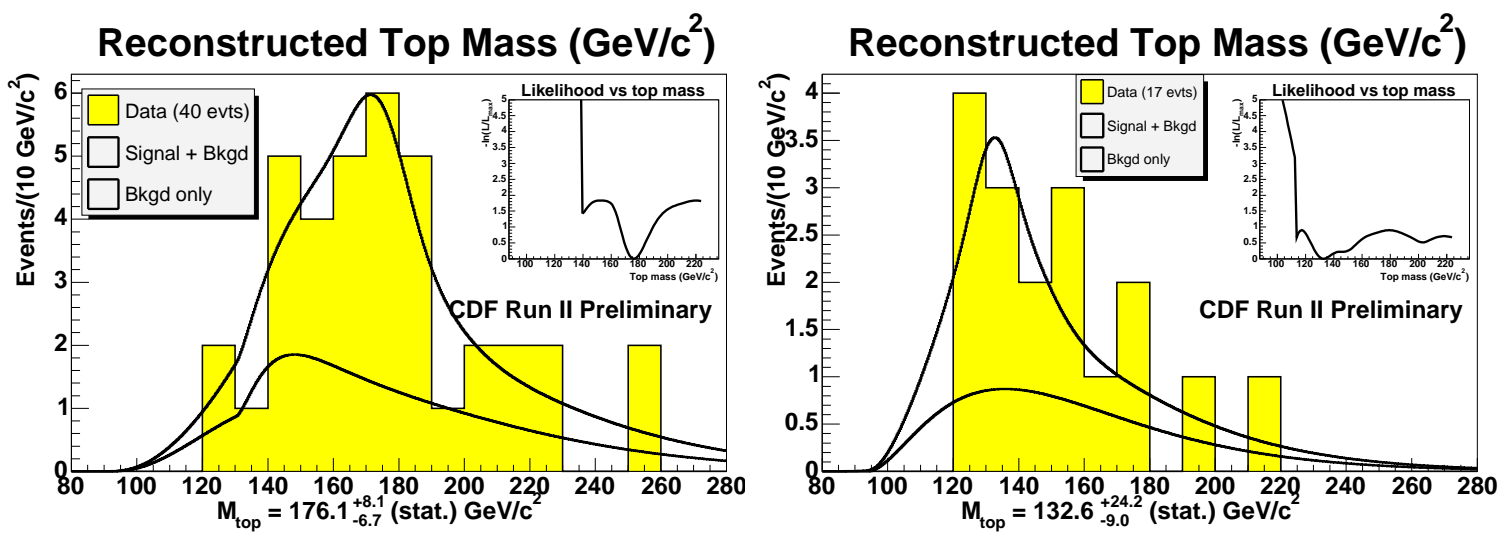

Likelihood vs top mass
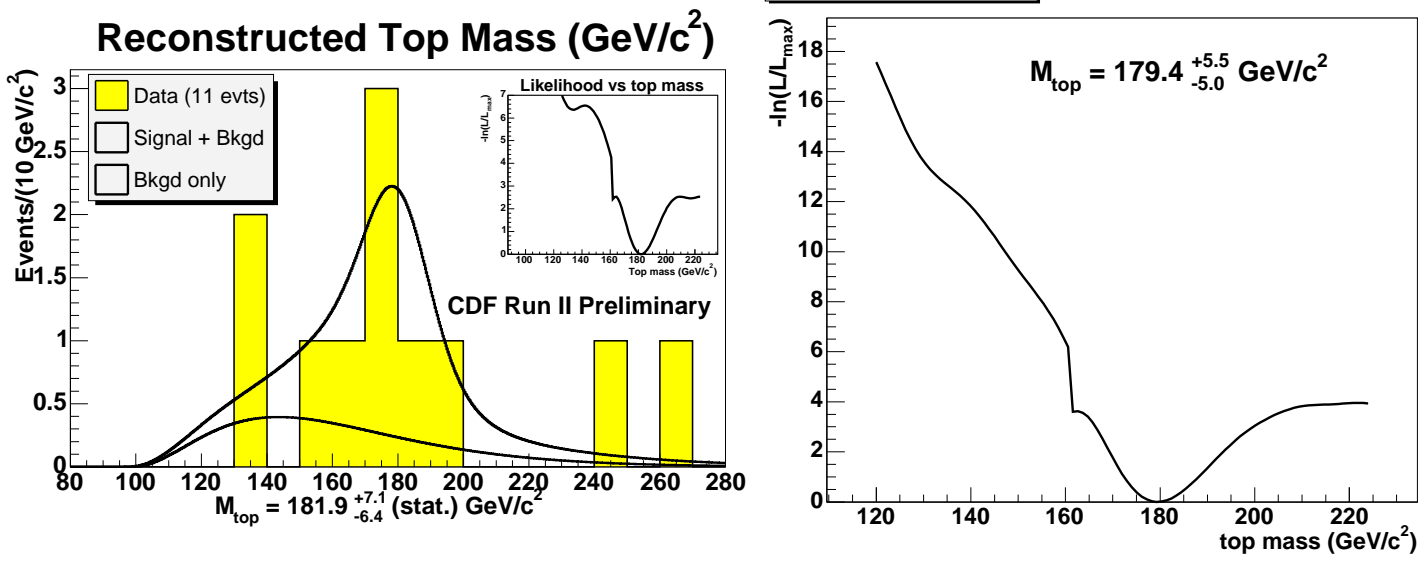

Figure 11.3: Result of the likelihood fit to the data sample for 0 tag (top-left), 1 tag (topright), 2 tag (bottom-left) and the combined fit of these three channels (bottom-right). The background constraint is turned off. The histogram shows the reconstructed mass of the data events for each sample. The distribution shape and the background contribution corresponding to the best fit are shown in lines. In the top-right box of each plot is the likelihood curve used in the measurement of the mass of the top quark. For the combined fit, we only show the likelihood curve.

Summarized in Table 11.5 are the results of the likelihood fit both with and without the background constraint. The results between the two likelihood fits are consistent for each sample, except for the background fraction of 1 tag sample in the combined fit. In fact, the top quark mass measured in the 1 tag sample in both constrained and unconstrained fit is much smaller 
than those in other samples. This is clearly due to the fact that the reconstructed masses of the 1 tag events are distributed in lower mass regions than any other channel. We will consider this further in Section 11.5.

\subsection{Check of the Result with Pseudo-Experiments}

The validity of the center value for the top quark mass measured in the previous section can not be checked by any results based on Monte Carlo studies. The Monte Carlo studies rather take the top quark mass as the input, which has no presumed value. The behavior of the statistical error, on the other hand, can be studied as a function of the top quark mass in the Monte Carlo studies. We can compare the statistical error of the measurement in data to the distribution obtained in pseudo-experiments.

We use a set of pseudo-experiments assuming a top quark mass $175 \mathrm{GeV} / c^{2}$, which is close to our measured center value in the combined channel $\left(177.2 \mathrm{GeV} / c^{2}\right)$. The distribution of the upper and lower statistical errors estimated in likelihood fit is shown in Figures 11.4, 11.5, 11.6 and 11.7 for $0,1,2$ tag sample and combined fit, respectively. The arrow in the plot indicates the estimated statistical error on the data measurement.

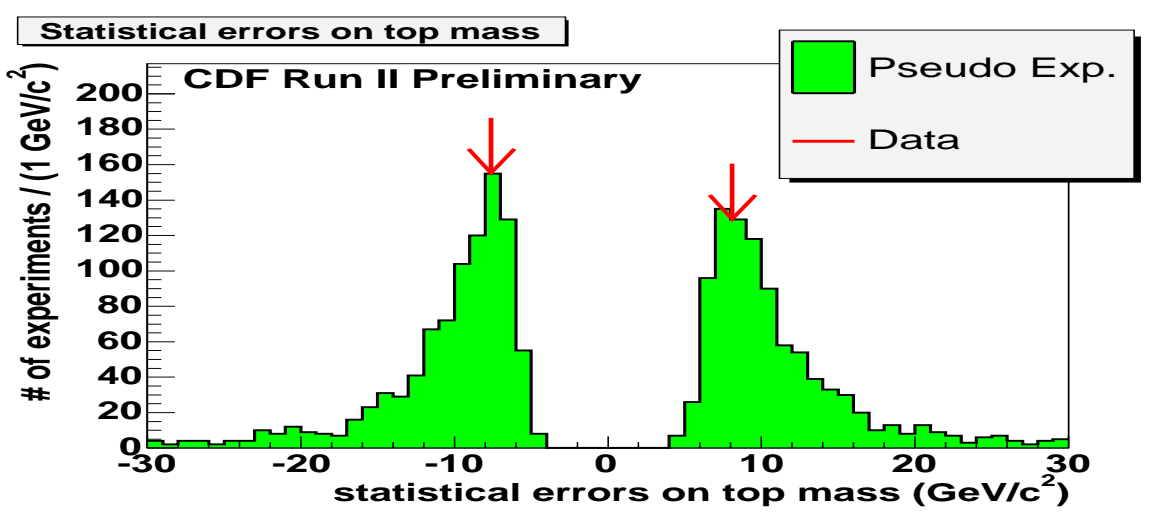

Figure 11.4: Distribution of the upper and lower statistical errors on top quark mass for 0 tag sample measurement obtained in 1000 pseudo-experiments assuming top mass of $175 \mathrm{GeV} / c^{2}$. The arrow indicates the value in data measurement.

The distribution of the pseudo-experiments in these figures can be interpreted as the probability distribution of the upper and lower statistical errors. The statistical errors of the 0 and 2 tag samples and the combined fit are reasonable because they are all within the probable regions. On the other hand, the upper error for the 1 tag sample measurement is unexpectedly large. In fact, 954 out of the 1000 pseudo experiments had the estimated upper statistical error smaller than that of the measurement in data. This means that we are statistically unlucky concerning the 1 tag sample. One possibility could be a concentration of the $W+3.5 j$ jets events in low mass 


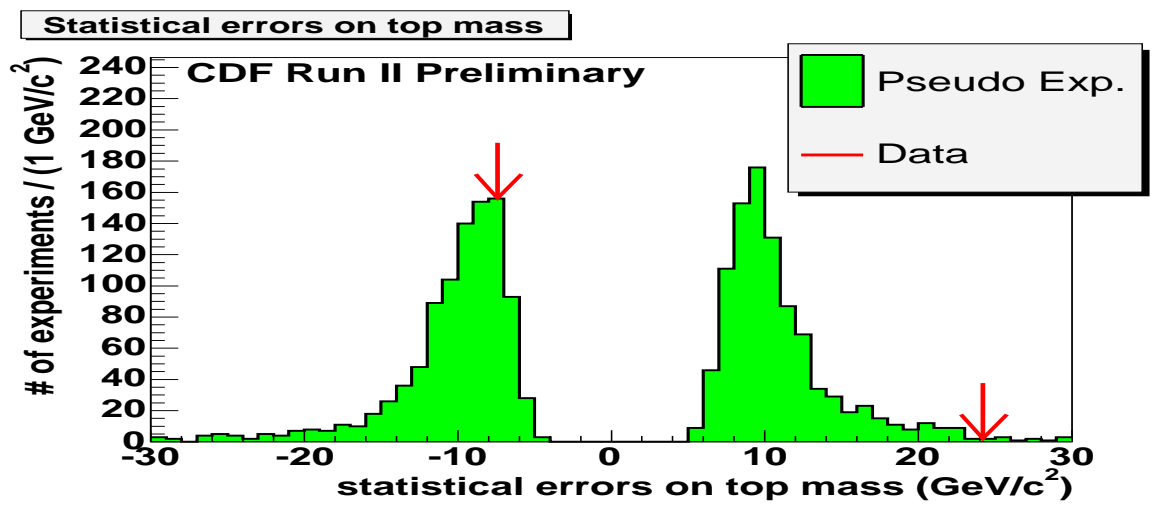

Figure 11.5: Distribution of the upper and lower statistical errors on top quark mass for 1 tag sample measurement obtained in 1000 pseudo-experiments assuming top mass of $175 \mathrm{GeV} / c^{2}$. The arrow indicates the value in data measurement.

region as shown in next section.

\subsection{Investigation into the 1 Tag Sample}

As one can see in Table 11.3, the reconstructed masses of the 3.5 jet events in the 1 tag sample are concentrated in low mass region. It would be interesting to know whether these events are responsible for the unexpectedly low value of the top quark mass measured in the 1 tag sample, although the results described in Section 11.3 stays our nominal results.

We try an alternative likelihood fit only with the 4 jet events in the tagged samples. The results are shown in Figure 11.8. The 0 tag sample is exactly the same as in Section 11.3. The 1 tag sample provide the fit mass of $171.6_{-14.8}^{+17.4} \mathrm{GeV} / c^{2}$ with ten candidate events, and the 2 tag sample gives $183.6{ }_{-6.1}^{+6.6} \mathrm{GeV} / c^{2}$ using nine candidate events. The combined likelihood fit of the three samples provides $180.3{ }_{-4.9}^{+5.3} \mathrm{GeV} / c^{2}$.

\subsection{Scale Factor for the Statistic Errors}

As described in Chapter 9, the width of our pull distribution is not consistent with 1 . This suggests that the statistical error calculated in the likelihood fit might be an underestimate. Therefore, we introduce a scale factor for the statistical error in order to obtain a correct statistical error. We define the scale factor so that the fraction of the pseudo-experiments which have the input top mass within the statistical errors around the output center values becomes $68 \%$. We use the set of 1000 pseudo-experiments assuming the top quark mass of $175 \mathrm{GeV} / c^{2}$, which was used in Section 11.4.

The scale factors were found to be 1.043 and 1.019 for the 2 tag sample and the combined 


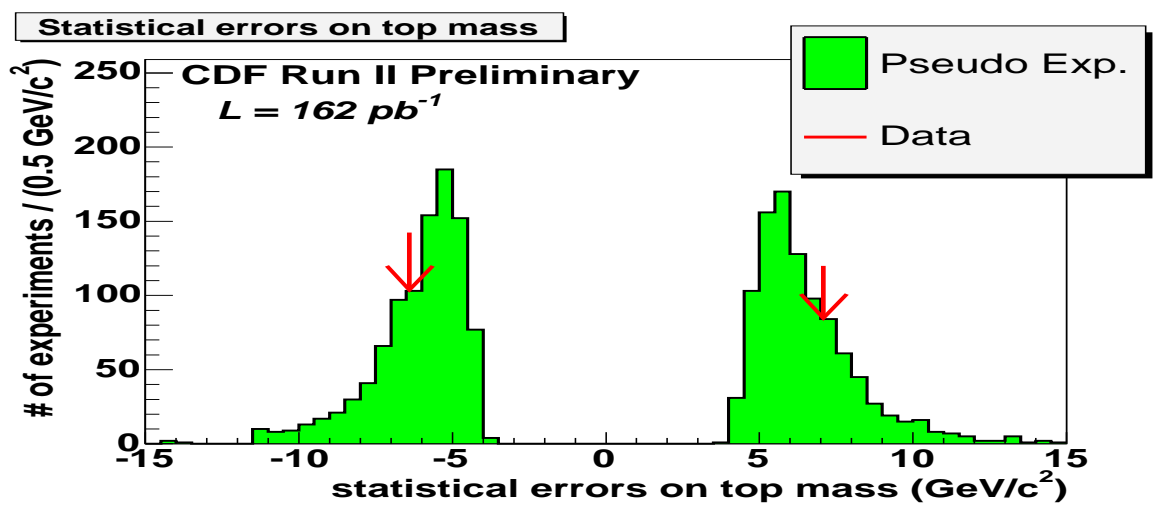

Figure 11.6: Distribution of the upper and lower statistical errors on top quark mass for 2 tag sample measurement obtained in 1000 pseudo-experiments assuming top mass of $175 \mathrm{GeV} / c^{2}$. The arrow indicates the value in data measurement.

fit, respectively. Applying these scale factors, our fit results are $180.9{ }_{-6.0}^{+6.4}$ (stat) $\mathrm{GeV} / \mathrm{c}^{2}$ for 2 tag sample and $177.2_{-4.7}^{+4.9}$ (stat) $\mathrm{GeV} / \mathrm{c}^{2}$ for the combined fit. 


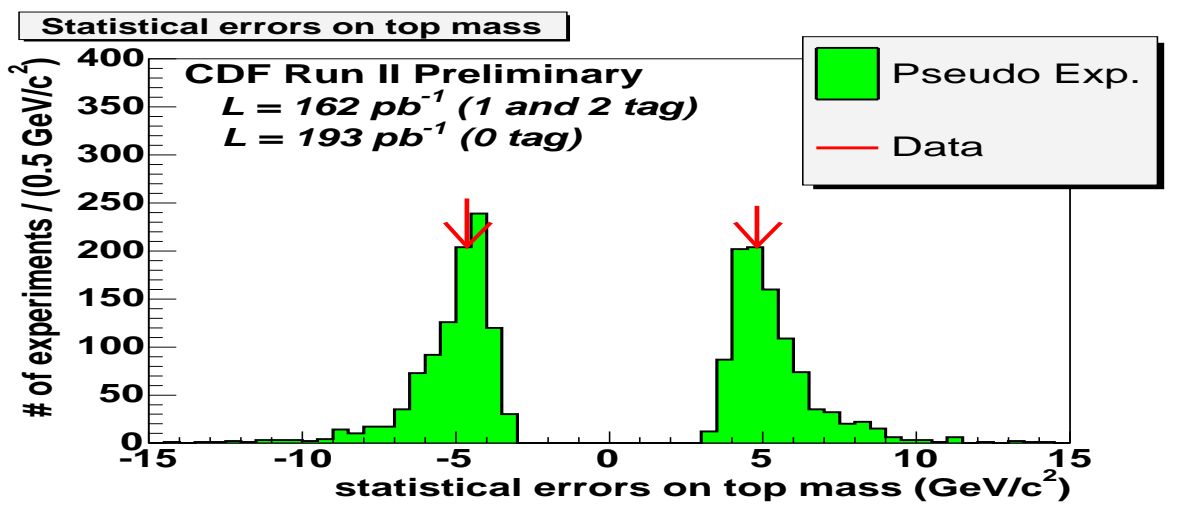

Figure 11.7: Distribution of the upper and lower statistical errors on top quark mass for measurement by combined fit obtained in 1000 pseudo-experiments assuming top mass of $175 \mathrm{GeV} / c^{2}$. The arrow indicates the value in data measurement.
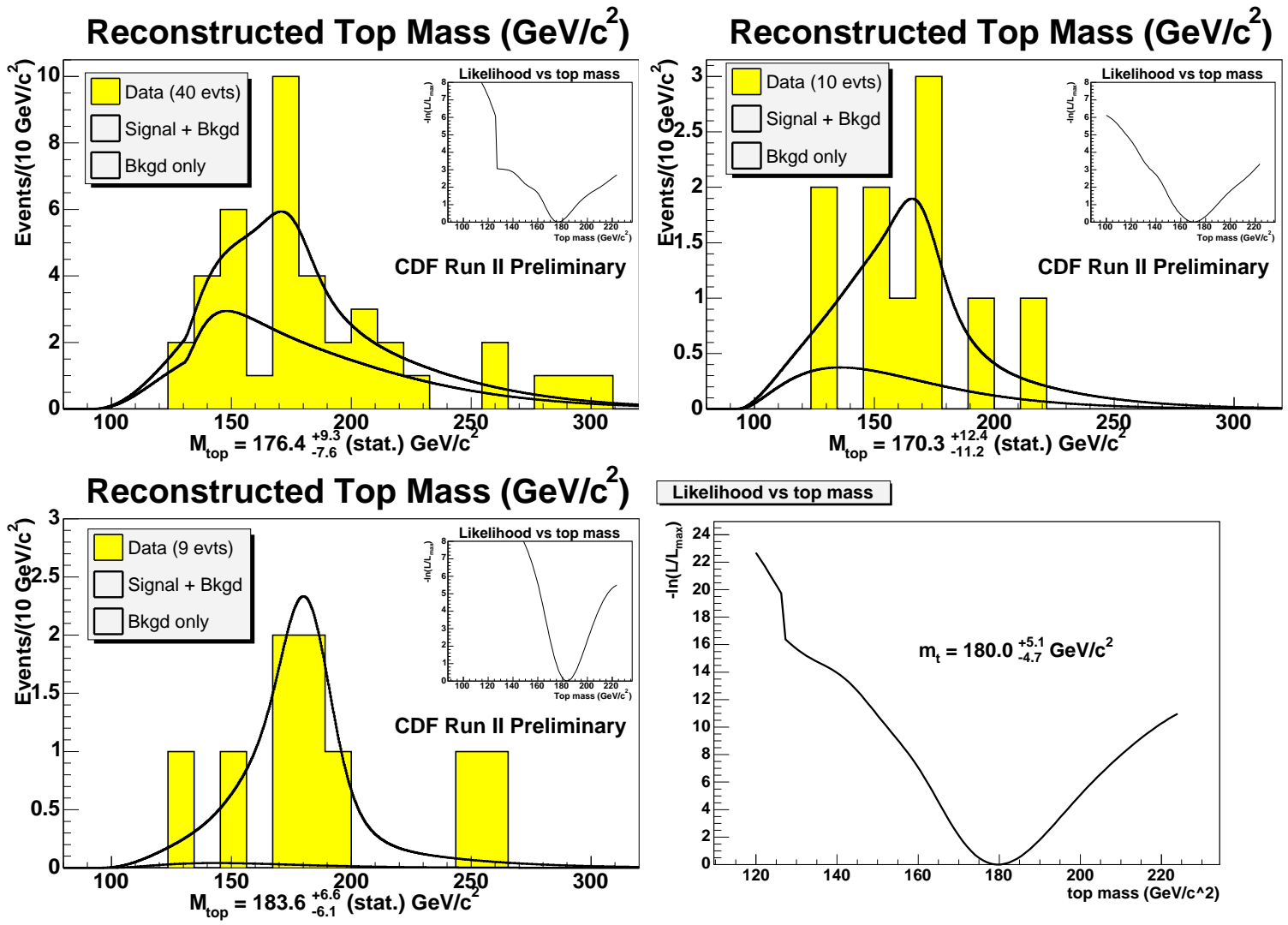

Figure 11.8: Fit results with background constrained using only 4 jet events in the tagged samples. Top left : 0 tag sample, top right : 1 tag sample, bottom left : 2 tag sample, bottom right : combined fit of the three samples. 


\begin{tabular}{|c|c|c|c|c|c|c|c|c|c|c|c|c|}
\hline run & event & $n_{j}$ & 1 & $\begin{array}{r}p_{t}^{l} \\
\mathrm{GeV}\end{array}$ & $\begin{array}{r}\not_{T} \\
\mathrm{GeV}\end{array}$ & $\begin{array}{c}E_{T}^{j 1} \\
\mathrm{GeV}\end{array}$ & $\begin{array}{c}E_{T}^{j 2} \\
\mathrm{GeV}\end{array}$ & $\begin{array}{c}E_{T}^{j 3} \\
\mathrm{GeV}\end{array}$ & $\begin{array}{c}E_{T}^{j 4} \\
\mathrm{GeV}\end{array}$ & $\chi^{2}$ & $\begin{array}{r}m_{t} \\
\mathrm{GeV}\end{array}$ & $\begin{array}{r}m_{j j} \\
\mathrm{GeV}\end{array}$ \\
\hline 141597 & 1353293 & 3.5 & CMUP & 51.2 & 30.9 & 54.4 & $42.9^{S J}$ & 35.8 & 13.2 & 3.4 & 135.2 & 110.7 \\
\hline 145036 & 245760 & 4 & CMUP & 28.2 & 67.4 & $101.9^{S}$ & 67.5 & 20.9 & 18.7 & 5.3 & 175.9 & 58.8 \\
\hline 148153 & 6088 & 3.5 & CEM & 54.9 & 48.2 & $71.9^{S}$ & $49.4^{J}$ & 19.2 & 9.4 & 0.3 & 144.3 & 80.5 \\
\hline 149387 & 2551061 & 3.5 & CEM & 66.8 & 29.7 & $37.9^{S J}$ & 28.9 & 17.2 & 8.8 & 4.2 & 122.0 & 58.8 \\
\hline 152266 & 3554 & 4 & CMUP & 30.6 & 40.2 & 67.2 & $45.8^{S J}$ & 19.8 & 16.2 & 0.6 & 153.6 & 89.4 \\
\hline 153738 & 2083102 & 4 & CEM & 23.0 & 67.2 & $107.0^{S J}$ & 63.2 & 57.9 & 24.8 & 2.6 & 216.8 & 105.1 \\
\hline 155345 & 3194866 & 3.5 & CEM & 26.4 & 61.9 & $46.3^{S J}$ & 45.4 & 16.2 & 8.9 & 3.8 & 122.9 & 109.4 \\
\hline 160153 & 1270879 & 4 & CEM & 93.2 & 52.1 & 92.5 & $53.2^{S}$ & 28.9 & 19.4 & 0.2 & 170.2 & 84.7 \\
\hline 161379 & 494836 & 4 & CEM & 155.4 & 29.7 & 102.8 & 40.6 & $32.3^{S}$ & 16.3 & 1.3 & 152.8 & 78.8 \\
\hline 161633 & 1571961 & 4 & CEM & 48.6 & 36.8 & 100.5 & $54.5^{S J}$ & 26.3 & 16.0 & 2.6 & 134.5 & 100.8 \\
\hline 162423 & 261933 & 4 & CEM & 73.3 & 30.5 & $40.0^{S J}$ & 31.2 & 31.1 & 16.6 & 0.9 & 129.6 & 73.4 \\
\hline 162519 & 931923 & 3.5 & CMX & 72.6 & 33.8 & $48.9^{S J}$ & 24.9 & 18.3 & 12.9 & 3.3 & 131.1 & 63.3 \\
\hline 162631 & 7109631 & 4 & CMX & 84.5 & 22.3 & 76.1 & 38.8 & $20.0^{S J}$ & 15.4 & 4.5 & 169.8 & 99.3 \\
\hline 164274 & 2932602 & 3.5 & CMUP & 77.6 & 24.2 & $37.9^{S J}$ & 32.4 & 26.4 & 10.8 & 1.3 & 148.6 & 95.1 \\
\hline 164819 & 2297394 & 4 & CEM & 31.5 & 46.8 & 40.9 & $39.3^{S J}$ & 17.6 & 15.7 & 0.7 & 159.2 & 90.3 \\
\hline 165314 & 1155563 & 3.5 & CMUP & 28.8 & 12.6 & $56.3^{J}$ & $22.8^{S J}$ & 20.7 & 10.4 & 1.0 & 126.4 & 92.6 \\
\hline 166007 & 498553 & 4 & CEM & 44.7 & 52.7 & $70.1^{S J}$ & 61.1 & 58.0 & 15.2 & 2.9 & 190.5 & 109.6 \\
\hline 155320 & 480816 & 4 & CEM & 99.4 & 28.1 & $54.1^{S J}$ & 34.0 & 21.0 & 17.6 & 41.6 & 146.2 & 39.1 \\
\hline 156116 & 6116596 & 4 & CEM & 25.3 & 28.2 & $74.5^{S J}$ & $57.1^{S J}$ & $36.6^{J}$ & 29.3 & 366.8 & 231.7 & 20.7 \\
\hline 160591 & 894406 & 4 & CMUP & 114.8 & 110.5 & $156.4^{J}$ & $70.3^{S J}$ & 61.4 & 17.6 & 9.6 & 232.2 & 92.5 \\
\hline 161013 & 111162 & 4 & CEM & 78.6 & 71.9 & $109.6^{S J}$ & 99.5 & 22.4 & 20.0 & 9.6 & 172.6 & 137.3 \\
\hline 161678 & 5912849 & 4 & CEM & 27.6 & 54.7 & $102.9^{S J}$ & 80.0 & 40.5 & 27.1 & 10.3 & 200.0 & 128.1 \\
\hline 165313 & 1770456 & 3.5 & CMUP & 112.0 & 22.9 & $102.7^{S J}$ & 22.7 & 15.2 & 13.4 & 20.9 & 137.8 & 43.7 \\
\hline 166805 & 2534588 & 4 & CMUP & 59.1 & 56.8 & $131.1^{S}$ & 37.1 & 29.1 & 22.1 & 13.6 & 172.1 & 74.1 \\
\hline 167139 & 1191211 & 4 & CMX & 31.2 & 63.5 & $62.5^{S J}$ & $42.3^{J}$ & $40.2^{S J}$ & 23.6 & 13.6 & 175.0 & 56.4 \\
\hline 167551 & 7969376 & 4 & $\mathrm{CEM}$ & 77.2 & 54.9 & $79.6^{S J}$ & $50.4^{J}$ & $31.7^{S J}$ & 25.0 & 173.6 & 278.0 & 40.9 \\
\hline
\end{tabular}

Table 11.3: The twenty-six events are selected as 1 tagged candidates, including the events rejected in 2 tag event selection. Nine events shown in the bottom failed to pass the $\chi^{2}$ cut. Remaining seventeen events are to be used in the mass measurement for the 1 tag sample. Jets with their $E_{T}$ 's tagged with ${ }^{S}$ are SECVTX tagged and with ${ }^{J}$ are Jet Probability tagged. The jet $E_{T}$ shown here are after the generic correction at level 5 . 


\begin{tabular}{|c|c|c|c|c|c|c|c|c|c|c|c|c|}
\hline run & event & $n_{j}$ & 1 & $\begin{array}{r}p_{t}^{l} \\
\mathrm{GeV}\end{array}$ & $\begin{array}{r}\ddot{E}_{T} \\
\mathrm{GeV}\end{array}$ & $\begin{array}{c}E_{T}^{j 1} \\
\mathrm{GeV}\end{array}$ & $\begin{array}{c}E_{T}^{j 2} \\
\mathrm{GeV}\end{array}$ & $\begin{array}{c}E_{T}^{j 3} \\
\mathrm{GeV}\end{array}$ & $\begin{array}{c}E_{T}^{j 4} \\
\mathrm{GeV}\end{array}$ & $\chi^{2}$ & $\begin{array}{r}m_{t} \\
\mathrm{GeV} \\
\end{array}$ & $\begin{array}{r}m_{j j} \\
\mathrm{GeV} \\
\end{array}$ \\
\hline 144883 & 693354 & 4 & $C E M$ & 46.8 & 31.1 & 49.8 & 40.1 & 28.1 & 26.3 & 0.4 & 142.3 & 74.3 \\
\hline 151237 & 191785 & 4 & $C M U P$ & 36.1 & 30.3 & 63.6 & 33.8 & 33.4 & 21.9 & 0.1 & 145.3 & 79.3 \\
\hline 152743 & 881536 & 4 & $C E M$ & 40.2 & 62.0 & 44.5 & 28.5 & 28.1 & 26.1 & 0.7 & 183.4 & 79.9 \\
\hline 153345 & 1121439 & 4 & $C E M$ & 42.2 & 11.1 & 134.7 & 92.5 & 72.0 & 25.4 & 0.1 & 193.6 & 80.3 \\
\hline 153389 & 3687166 & 4 & $C M U P$ & 49.5 & 89.4 & 114.0 & 34.3 & 23.5 & 21.8 & 0.3 & 170.1 & 75.9 \\
\hline 154452 & 4848470 & 4 & $C E M$ & 111.3 & 44.1 & 63.8 & 53.5 & 52.5 & 27.7 & 3.3 & 222.4 & 73.2 \\
\hline 156457 & 13182 & 4 & $C E M$ & 33.2 & 69.2 & 63.2 & 33.6 & 31.7 & 31.2 & 0.8 & 168.9 & 91.0 \\
\hline 160150 & 217608 & 4 & $C E M$ & 67.9 & 25.6 & 94.4 & 63.4 & 32.3 & 22.4 & 0.5 & 210.2 & 87.6 \\
\hline 160438 & 628311 & 4 & $C E M$ & 39.3 & 43.4 & 90.0 & 52.6 & 36.3 & 32.5 & 0.7 & 202.8 & 90.6 \\
\hline 160441 & 1593640 & 4 & $C E M$ & 38.4 & 19.4 & 46.9 & 36.2 & 30.5 & 24.3 & 0.1 & 213.0 & 77.3 \\
\hline 160441 & 3910866 & 4 & $C E M$ & 109.2 & 51.9 & $89.5^{J}$ & 85.2 & $48.2^{J}$ & 45.0 & 2.1 & 181.8 & 72.3 \\
\hline 160594 & 290458 & 4 & $C E M$ & 96.0 & 46.2 & 52.6 & 40.9 & 32.0 & 25.2 & 0.6 & 167.3 & 87.4 \\
\hline 160988 & 3755474 & 4 & $C E M$ & 36.0 & 46.2 & 43.0 & 34.2 & 27.1 & 21.1 & 0.1 & 152.1 & 76.5 \\
\hline 161409 & 259495 & 4 & $C M U P$ & 29.6 & 30.1 & 52.5 & 44.0 & 34.3 & 28.8 & 4.0 & 152.6 & 112.6 \\
\hline 161414 & 68227 & 4 & $C E M$ & 51.8 & 61.1 & 95.8 & 75.5 & 30.0 & 26.5 & 5.3 & 173.4 & 93.1 \\
\hline 161792 & 391660 & 4 & $C E M$ & 46.7 & 55.0 & 45.1 & 43.4 & $30.7^{J}$ & 24.9 & 0.1 & 151.7 & 81.9 \\
\hline 161823 & 141809 & 4 & $C E M$ & 65.6 & 128.7 & 149.7 & 73.8 & 50.5 & 21.8 & 2.4 & 299.8 & 75.1 \\
\hline 162519 & 907935 & 4 & $C E M$ & 41.5 & 18.7 & 67.5 & 65.4 & 51.2 & 24.1 & 0.5 & 187.6 & 87.6 \\
\hline 162631 & 10198058 & 4 & $C E M$ & 54.8 & 70.2 & 103.2 & 70.1 & $42.9^{J}$ & 33.7 & 1.8 & 147.1 & 70.9 \\
\hline 162837 & 1447297 & 4 & $C M X$ & 80.6 & 39.9 & 72.5 & 52.8 & 43.6 & 26.3 & 1.5 & 169.9 & 92.1 \\
\hline 164110 & 954852 & 4 & $C E M$ & 30.9 & 42.1 & 48.2 & 46.5 & 33.5 & 33.1 & 0.3 & 173.8 & 86.7 \\
\hline 164819 & 1242550 & 4 & $C E M$ & 154.7 & 34.5 & 127.1 & 51.8 & $25.5^{J}$ & 21.3 & 1.5 & 176.4 & 90.8 \\
\hline 164822 & 901763 & 4 & $C M U P$ & 82.0 & 10.4 & 123.5 & 82.6 & 30.9 & 26.6 & 7.2 & 168.2 & 98.9 \\
\hline 165121 & 9869922 & 4 & $C M U P$ & 30.3 & 83.6 & $100.2^{J}$ & 54.7 & 41.5 & 24.7 & 1.1 & 153.0 & 89.6 \\
\hline 165271 & 2372808 & 4 & $C E M$ & 57.0 & 42.6 & 60.2 & 36.5 & 22.0 & 21.1 & 4.1 & 135.1 & 87.7 \\
\hline 166063 & 1026436 & 4 & $C M X$ & 27.2 & 31.3 & 37.8 & 35.6 & 24.0 & 22.7 & 0.1 & 128.2 & 78.1 \\
\hline 166529 & 4938 & 4 & $C M U P$ & 41.9 & 43.6 & $59.6^{J}$ & 27.4 & 26.3 & 24.7 & 0.5 & 141.8 & 87.6 \\
\hline 166614 & 440459 & 4 & $C M U P$ & 39.4 & 19.1 & 35.5 & 34.1 & 28.2 & 21.2 & 1.4 & 126.0 & 73.3 \\
\hline 166717 & 2288892 & 4 & $C M U P$ & 73.7 & 62.4 & 73.7 & 54.3 & $51.9^{J}$ & 36.9 & 0.4 & 202.2 & 75.9 \\
\hline 167023 & 7095000 & 4 & $C M U P$ & 48.9 & 27.2 & 194.4 & 77.2 & 69.7 & 22.1 & 4.9 & 286.1 & 78.3 \\
\hline 167325 & 1861198 & 4 & $C E M$ & 20.8 & 91.6 & 89.4 & 53.1 & 25.0 & 25.0 & 0.4 & 256.7 & 82.2 \\
\hline 167549 & 208319 & 4 & $C E M$ & 90.5 & 30.0 & 121.1 & 24.4 & 23.8 & 23.5 & 0.5 & 189.8 & 85.8 \\
\hline 167551 & 3626393 & 4 & $C M U P$ & 25.3 & 36.0 & 78.9 & 41.8 & 31.4 & $26.1^{J}$ & 0.6 & 160.2 & 72.7 \\
\hline 167715 & 557934 & 4 & $C E M$ & 47.2 & 25.1 & 48.8 & 48.1 & 31.5 & 23.4 & 3.8 & 259.6 & 62.5 \\
\hline 167849 & 4509595 & 4 & $C M U P$ & 45.9 & 74.2 & 78.7 & 44.0 & 34.0 & 28.4 & 2.7 & 171.2 & 72.7 \\
\hline 167977 & 6961724 & 4 & $C E M$ & 34.5 & 35.0 & 34.1 & 32.6 & 24.4 & 24.3 & 1.5 & 171.1 & 89.9 \\
\hline 168125 & 398014 & 4 & $C M U P$ & 55.5 & 41.6 & 112.3 & 53.9 & 44.1 & 23.7 & 4.8 & 292.9 & 67.5 \\
\hline 168563 & 2395692 & 4 & $C E M$ & 31.8 & 45.2 & 66.3 & 60.5 & 31.7 & 26.3 & 0.3 & 146.3 & 83.9 \\
\hline 168599 & 6653973 & 4 & $C E M$ & 32.6 & 46.2 & 70.1 & 64.8 & $61.4^{S J}$ & 43.3 & 0.1 & 221.0 & 82.0 \\
\hline 168889 & 1456443 & 4 & $C M U P$ & 50.8 & 36.1 & 46.5 & 41.4 & $39.1^{S J}$ & 35.5 & 0.0 & 180.6 & 78.4 \\
\hline 148857 & 462914 & 4 & $C M U P$ & 332.3 & 320.1 & 99.2 & 80.2 & 30.1 & 22.0 & 13.1 & 192.2 & 107.1 \\
\hline 151844 & 2846111 & 4 & $C M U P$ & 34.0 & 57.2 & 113.2 & 47.8 & 29.1 & 27.6 & 12.6 & 167.8 & 141.1 \\
\hline
\end{tabular}

Table 11.4: Forty-two events survived our kinematic cuts for the 0 tag selection criteria, including the $E_{T}^{j 4}>21 \mathrm{GeV}$. Two of these events were rejected at our $\chi^{2}$ cut, which are shown at the bottom of this table. Remaining forty events are used as 0 tag sample and are used for the mass measurement. 


\begin{tabular}{|c|c|c|c|c|c|}
\hline & & for 0tag sample & for 1tag sample & for $2 \mathrm{tag}$ sample & for combined sample \\
\hline constraint & $\begin{array}{l}\text { Best fit } \\
\text { input bkgd frac. } \\
\text { output bkgd frac. }\end{array}$ & $\begin{array}{c}176.4_{-7.6}^{+9.3} \\
0.61 \pm 0.18 \\
0.57 \pm 0.16\end{array}$ & $\begin{array}{l}132.8_{-7.4}^{+23.4} \\
0.38 \pm 0.07 \\
0.38 \pm 0.32\end{array}$ & $\begin{array}{c}180.9_{-5.8}^{+6.1} \\
0.025 \pm 0.018 \\
0.031 \pm 0.015\end{array}$ & $\begin{array}{c}177.2_{-4.6}^{+4.8} \\
- \\
0.57 \pm 0.16(0 \mathrm{tag}) \\
0.41 \pm 0.07(1 \mathrm{tag}) \\
0.026 \pm 0.005(2 \mathrm{tag})\end{array}$ \\
\hline unconstrained & $\begin{array}{c}\text { Best fit } \\
\text { output bkgd frac. }\end{array}$ & $\begin{array}{c}176.1_{-6.7}^{+8.1} \\
0.39 \pm 0.32\end{array}$ & $\begin{array}{l}132.6_{-9.0}^{+24.2} \\
0.41 \pm 0.41\end{array}$ & $\begin{array}{c}181.9_{-6.4}^{+7.1} \\
0.27 \pm 0.30\end{array}$ & $\begin{array}{c}179.4{ }_{-5.0}^{+5.4} \\
0.42 \pm 0.31(0 \mathrm{tag}) \\
1-\text { at limit }(1 \mathrm{tag}) \\
0.26 \pm 0.30(2 \mathrm{tag})\end{array}$ \\
\hline
\end{tabular}

Table 11.5: Summary of the likelihood fit with and without constraint on the number of background events. The measured top quark mass and the background fraction is shown for each likelihood fit. The input background fraction is also shown for the fit with the background constraint.
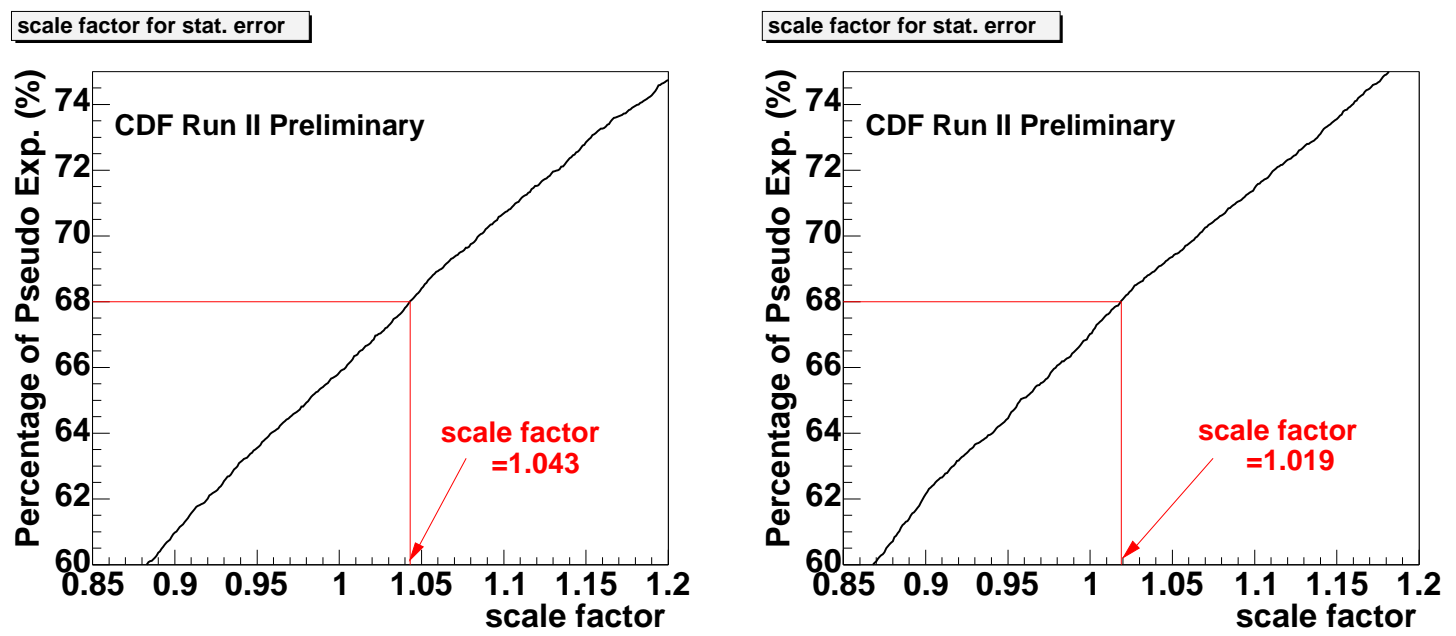

Figure 11.9: The percentage of the pseudo-experiments which have the input top mass within the statistical errors around the output center values (on the vertical axis). The scale factor applied to the statistical error from the likelihood fit is plotted on the horizontal axis. Left plot is for the 2 tag sample and the right plot is for the combined fit. 


\section{Chapter 12}

\section{Conclusion}

The top quark mass was measured in the lepton + jets decay of $t \bar{t}$ creation events in $p-\bar{p}$ collisions at $\sqrt{s}=1.96 \mathrm{TeV}$. The data was taken with the Collider Detector at Fermilab (CDF) in Run II. We subdivided the candidate events into three categories due to the number of $b$ tagged jets in the event : 0,1 and 2 tag samples. Two different $b$-tagging algorithms were used in our analysis : SECVTX algorithm and Jet Probability algorithm. We optimized the cut threshold in the Jet Probability algorithm in order to minimize statistical uncertainty on the top quark mass measured in the combined fit of the three samples. As a result, we reduced the statistical uncertainty on the combined top mass measurement by $5 \%$.

We used data collected with CDF detector from March 2002 through August 2003. The integrated luminosity corresponding to the data used in this analysis was :

- $162 \mathrm{pb}^{-1}$ for 1 and 2 tag samples.

- $193 \mathrm{pb}^{-1}$ for 0 tag sample.

We found eleven, seventeen and forty $t \bar{t}$ candidate events in 2, 1 and 0 tag sample, respectively. We measured the mass of the top quark to be $180.9_{-6.0}^{+6.4}$ (stat) \pm 5.8 (syst) $\mathrm{GeV} / c^{2}$ using the eleven candidates in the 2 tag sample, and $177.2_{-4.7}^{+4.9}$ (stat) \pm 6.6 (syst) $\mathrm{GeV} / c^{2}$ by combining the three samples.

The CDF experiment using the Tevatron Run II has been eagerly studying the properties of the top quark, and other analysis methods have also been explored for the measurement of the top quark mass. Summarized in Figure 12.1 are the results from different analyses so far presented by the CDF Run II Collaboration. Study on combining results of different measurement methods have not been performed yet for these Run II results. The combined measurements by CDF and D0 Collaborations as well as the combined result of these two collaborations obtained in Run I are also shown in this figure. 


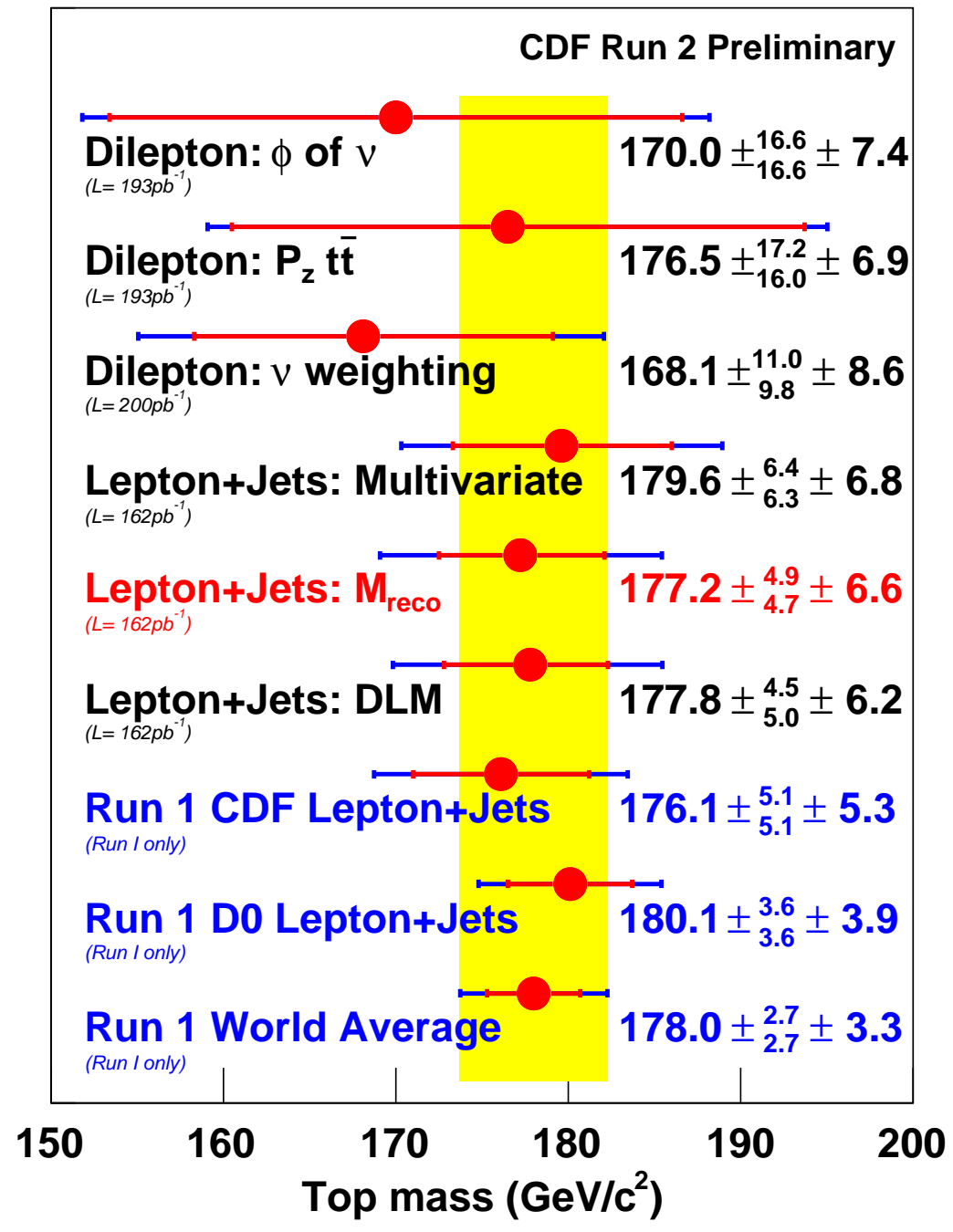

Figure 12.1: Results of top quark mass measurements using different analysis methods at CDF Run II. The result presented in this dissertation is referred to as $M_{\text {reco }}$. 


\section{Bibliography}

[1] F. Abe et al. [CDF Collaboration], Phys. Rev. Lett. 73, 225 (1994); Phys. Rev. D50, 2966 (1994); S. Abachi et al., Phys. Rev. Lett. 74 (1995) 2632.

[2] D. Gross, F. Wilczek, Phys. Rev. Lett. 30, 1343 (1973).

[3] M. Gell-Mann, Phys. Lett. 8, 214-215 (1964).

[4] R. P. Feynman, "QED. The Strange Theory of Light and Matter", Princeton Science Library (1985).

[5] F. Halzen, A. D. Martin, "Quarks And Leptons: An Introductory Course In Modern Particle Physics," John Wiley and Sons (1984).

[6] R. K. Ellis, W. J. Stirling and B. R. Webber, "QCD and collider physics," Camb. Monogr. Part. Phys. Nucl. Phys. Cosmol. 8, 1 (1996).

[7] M. Cacciari et al., JHEP 404 (2004) 68.

[8] N. Kidonakis and R. Vogt, Phys. Rev. D 68114014 (2003).

[9] S. Baroiant et al., Proceedings 4th International Workshop on Very High Multiplicity Physics, Alushta, Crimea, Ukraine, May (2003) FERMILAB-CONF-03/322-E.

[10] F. Abe et al., Phys. Rev. Lett. 82 (1999) 2808.

[11] B. Abbott et al., Phys. Rev. D60 (1999) 052001.

[12] T. Affolder et al., Phys. Rev. D60 (2001) 032003.

[13] B. Abbott et al., Phys. Rev. D58 (1998) 052001.

[14] F. Abe et al., Phys. Rev. Lett. 79 (1997) 1992.

[15] V.M. Abazov et al., Nature 429 (2004) 638. ; J.C. Estrada Vigil, arXiv:hep-ex/0302031.

[16] Tevatron Elecroweak Working Group, arXiv:hep-ex/0404010. http://tevewwg.fnal.gov/ 
[17] S. Willenbrock, arXiv:hep-ph/0211067.

[18] LEP Electroweak Working Group, Phys. Lett. B565 (2003) 61; Update August 2004: http://lepewwg.web.cern.ch/LEPEWWG.

[19] http://lephiggs.web.cern.ch/LEPHIGGS/www/Welcome.html

[20] J.F. de Troconiz and F. J. Yndurain, Phys. Rev. D65 (2002) 093002.

[21] F. Abe et al. [CDF Collaboration], Nucl. Instrum. Meth. A 271, 387 (1988) and FERMILAB-PUB-96/390-E.

[22] A. Sill et al., Nucl. Instrum. and Methods A447 (2000) 1.

[23] T. Affolder et al., Nucl. Instrum. and Methods 526 (2004) 249.

[24] F. Bedeschi et al. [CDF Collaboration], Nucl. Instrum. Meth. A 268, 50 (1988).

[25] L. Balka et al. [CDF Collaboration], Nucl. Instrum. Meth. A 267, 272 (1988).

[26] S. Bertolucci et al. [CDF Collaboration], Nucl. Instrum. Meth. A 267, 301 (1988).

[27] K. Byrum et al., Nucl. Instrum. Meth. A 364, 144 (1995).

[28] F. Abe et al. [CDF Collaboration], Phys. Rev. Lett. 73, 2662 (1994) [Erratum-ibid. 74, 1891 (1995)].

[29] K. Byrum et al. [CDF Collaboration], Nucl. Instrum. Meth. A 268, 46 (1988).

[30] G. Ascoli et al., Nucl. Instrum. Meth. A 268 (1988) 33.

[31] T. Dorigo et al., Nucl. Instrum. and Methods A461 (2001) 560.

[32] P. Canal et al., IEEE Trans. Nucl. Sci. 47 (2000) 240.

[33] W. Wagner et al., FERMILAB-CONF-02-269-E, International Europhysics Conference on High-Energy Physics (HEP 2001), Budapest, Hungary, 12-18 Jul 2001.

[34] B. Angelos et al. [CDF Collaboration], International Conference on Computing in HighEnergy Physics and Nuclear Physics (CHEP 2000), Padova, Italy, 7-11 Feb 2000.

[35] D. Glenzinski et al., CDF internal note 5973.

[36] J. Goldstein et al., CDF internal note 5970.

[37] T. Nelson et al., CDF internal note 6278.

[38] F. Abe et al., Phys. Rev. D45 (1992) 1448. 
[39] J-F Arguin et al., CDF internal note 6565.

[40] T. Affolder et al., Phys. Rev. D64 (2001) 032002.

[41] D. Acosta et al., submitted to Phys. Rev. D (2004).

[42] W. D. Acosta et al., CDF internal note 6315.

[43] D. Gerdes et al., CDF internal note 2023.

[44] A. Sukhanov et al., CDF internal note 6931.

[45] G. Gomez et al., CDF internal note 6913.

[46] G. Marchesini et al., Comp. Phys. Commun. 67 (1992) 465 ; G. Corcella et al., JHEP 0101 (2001) 010.

[47] T. Sjostrand et al., Comp. Phys. Commun. 135 (2001) 238.

[48] J. Pumplin et al., JHEP 0207 (2002) 012.

[49] M. L. Mangano et al., J. High Energy Phys. JHEP 07 (2003) 001.

[50] R. Brun and F. Carminati, CERN Programming Library Long Writeup W5013 (1993).

[51] G. Grindhammer, M. Rudowicz, and S. Peters, Nucl. Instrum. and Methods A290 (1990) 469.

[52] K. Bloom et al., CDF internal note 6844.

[53] E. Brubaker et al., CDF internal note 6845.

[54] G. Bellettini et al., CDF internal note 6845.

[55] J-F Arguin et al., CDF internal note 7139.

[56] H. Bachacou et al., CDF internal note 6904.

[57] F. James and M. Roos, Comput. Phys. Commun. 10 (1975) 343.

[58] M. Cacciari et al., arXiv:hep-ph/0303085.

[59] K. Bloom, CDF internal note 6559.

[60] S. Miller et al., CDF internal note 6907.

[61] A. Dominguez et al., CDF internal note 6533.

[62] B. Harris et al., Phys. Rev. D66 (2002) 054024. 
[63] J. Campbell and R. Ellis, Phys. Rev. D60 (1999) 113006.

[64] S-B Kim et al., CDF internal note 3007.

[65] U-K Yang et al., CDF internal note 6804.

[66] http//www-cdf.fnal.gov/internal/physics/top/run2mass/ISR_FSR 